\title{
Gesellschaft auf einen Blick 2009 OECD-SOZIALINDIKATOREN
}
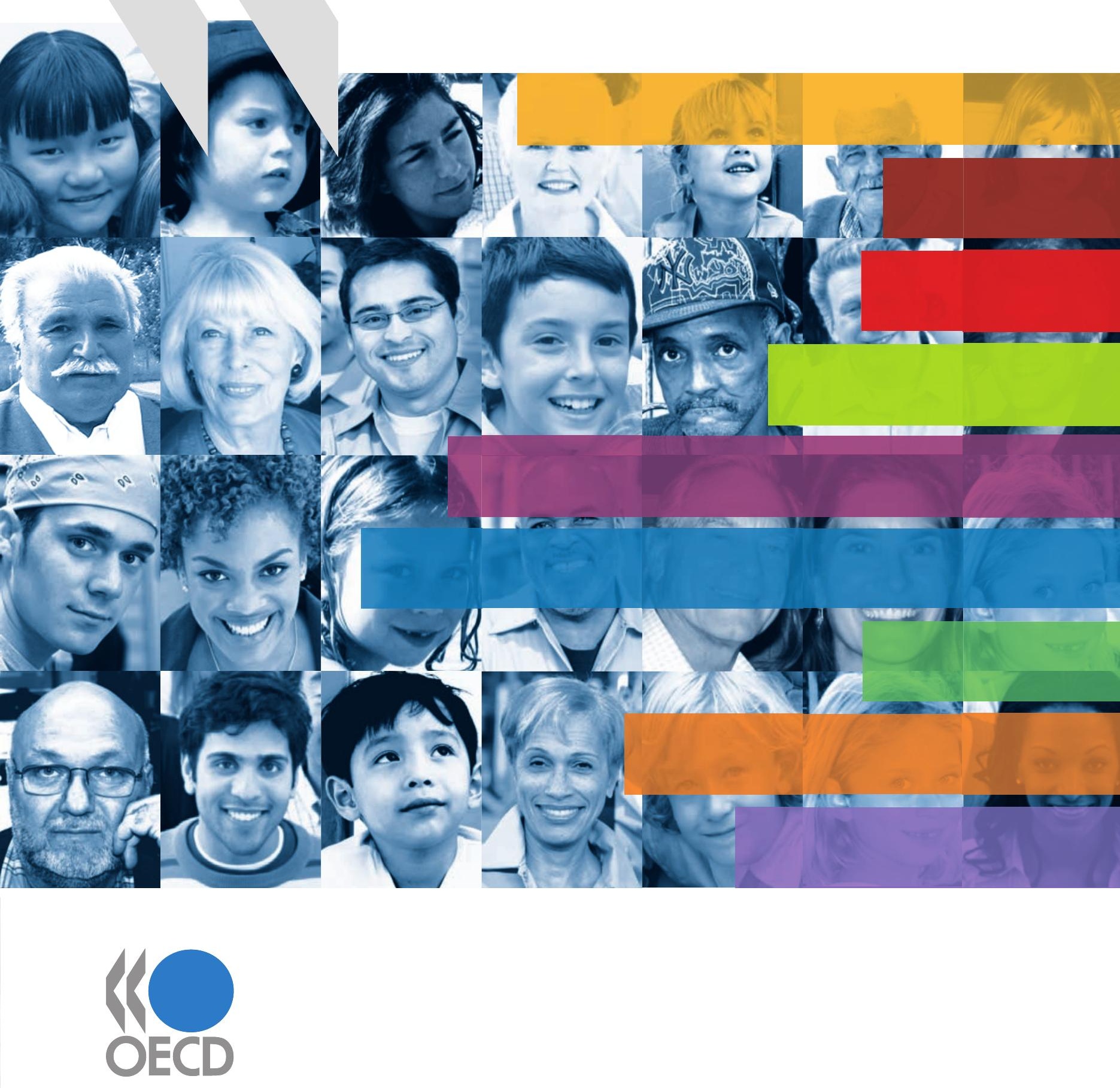



\title{
Gesellschaft \\ auf einer Blick 2009
}

\author{
OECD-SOZIALINDIKATOREN
}

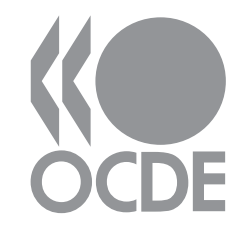




\section{ORGANISATION FÜR WIRTSCHAFTLICHE ZUSAMMENARBEIT UND ENTWICKLUNG}

Die OECD ist ein in seiner Art einzigartiges Forum, in dem die Regierungen von 30 demokratischen Staaten gemeinsam an der Bewältigung von Herausforderungen der Globalisierung im Wirtschafts-, Sozial- und Umweltbereich arbeiten. Die OECD steht auch in vorderster Linie bei den Bemühungen um ein besseres Verständnis der neuen Entwicklungen und durch sie ausgelöster Befürchtungen, indem sie Untersuchungen zu Themen wie Corporate Governance, Informationswirtschaft oder Bevölkerungsalterung durchführt. Die Organisation bietet den Regierungen einen Rahmen, der es ihnen ermöglicht, ihre Politikerfahrungen auszutauschen, nach Lösungsansätzen für gemeinsame Probleme zu suchen, empfehlenswerte Praktiken aufzuzeigen und auf eine Koordinierung nationaler und internationaler Politiken hinzuarbeiten.

Die OECD-Mitgliedstaaten sind: Australien, Belgien, Dänemark, Deutschland, Finnland, Frankreich, Griechenland, Irland, Island, Italien, Japan, Kanada, Korea, Luxemburg, Mexiko, Neuseeland, die Niederlande, Norwegen, Österreich, Polen, Portugal, Schweden, Schweiz, die Slowakische Republik, Spanien, die Tschechische Republik, Türkei, Ungarn, das Vereinigte Königreich und die Vereinigten Staaten. Die Kommission der Europäischen Gemeinschaften nimmt an den Arbeiten der OECD teil.

OECD Publishing sorgt dafür, dass die Ergebnisse der statistischen Analysen und der Untersuchungen der Organisation zu wirtschaftlichen, sozialen und umweltpolitischen Themen sowie die von den Mitgliedstaaten vereinbarten Übereinkommen, Leitlinien und Standards weite Verbreitung finden.

Das vorliegende Dokument wird unter der Verantwortung des Generalsekretärs der OECD veröffentlicht. Die darin zum Ausdruck gebrachten Meinungen und Argumente spiegeln nicht zwangsläufig die offizielle Einstellung der Organisation oder der Regierungen ihrer Mitgliedstaaten wider.

Originaltitel: Society at a Glance 2009: OECD Social Indicators - Panorama de la société 2009 : les indicateurs sociaux de l'OCDE.

Übersetzung durch den Deutschen Übersetzungsdienst der OECD.

Foto(s): Deckblatt: ๑ Floresco Productions/Cultura/Getty Images; ๑ Image Source/Corbis; ๑ PhotoAlto /Alamy; ๑ Thinkstock LLC/ Jupiterimages; ( Corbis/Inmagine ltb; Kapitel 4: ๑) Stockbyte/Getty Images; Kapitel 5: @ Maria Taglienti-Molinari/Brand X/ Corbis; Kapitel 6: ๑ Matthieu Spohn/PhotoAlto Agency RF Collections/Getty Images; Kapitel 7: ๑ Helen King/Corbis; Kapitel 8: () Daniel Boiteau/Fotolia.com

Korrigenda zu OECD-Veröffentlichungen sind verfügbar unter: www.oecd.org/publishing/corrigenda.

(c) OECD 2009

Die OECD gestattet das Kopieren, Herunterladen und Abdrucken von OECD-Inhalten für den eigenen Gebrauch sowie das Einfügen von Auszügen aus OECD-Veröffentlichungen, -Datenbanken und -Multimediaprodukten in eigene Dokumente, Präsentationen, Blogs, Websites und Lehrmaterialien, vorausgesetzt die OECD wird in geeigneter Weise als Quelle und Urheberrechtsinhaber genannt. Sämtliche Anfragen bezüglich Verwendung für öffentliche oder kommerzielle Zwecke bzw. Übersetzungsrechte sind zu richten an: rights@oecd.org. Die Genehmigung zur Kopie von Teilen dieser Publikation für den öffentlichen oder kommerziellen Gebrauch ist direkt einzuholen beim Copyright Clearance Center (CCC) unter info@copyright.com oder beim Centre français d'exploitation du droit de copie (CFC) unter contact@cfcopies.com. 


\section{Vorwort}

$D_{\text {ies }}$ ies ist die fünfte Ausgabe von Gesellschaft auf einen Blick, dem im zweijährigen Turnus erscheinenden Kompendium von Sozialindikatoren der OECD. Dieser Bericht soll der wachsenden Nachfrage nach quantitativen Daten über das soziale Wohlergehen und die entsprechenden Trends gerecht werden. In der Ausgabe 2009 werden einige in den Ausgaben 2001, 2003, 2005 und 2006 enthaltene Indikatoren aktualisiert. Es werden zudem einige neue hinzugefügt: Indikatoren für die Körpergröße, die Selbsteinschätzung des Gesundheitszustands, Risikoverhalten und aggressives Verhalten von Jugendlichen (Bullying). Zusätzlich wird eine Reihe von sozialen Leitindikatoren entwickelt, die einen Überblick über das soziale Wohlergehen und die entsprechenden Trends gibt. Der Bericht enthält auch einen Interpretationsleitfaden, der den Lesern das Verständnis des Aufbaus der OECD-Sozialindikatoren erleichtern soll, und ein Sonderkapitel über das Thema Freizeit im OECD-Raum. Detailliertere Informationen zu sämtlichen Indikatoren, auch solchen, die in dieser Ausgabe nicht enthalten sind, finden sich auf der entsprechenden OECD-Website (www.oecd.org) els/social/indicators/SAG).

Dieser Bericht wurde von Simon Chapple und Maxime Ladaique erstellt. Da er ein breites Spektrum von Themen anspricht, wäre er nicht möglich gewesen ohne die Mitwirkung zahlreicher Personen in- und außerhalb der OECD-Abteilung Sozialpolitik, darunter Francesca Colombo, Michael De Looper, Marco Mira d'Ercole, Justina Fischer, Michael Förster, Pauline Fron, Rie Fujisawa, David Jonathan Gonzales-Villascan, Ingrid Herrbach, Maria del Carmen Huerta, Herwig Immervoll, Gaetan Lafortune, Pascal Marianna, Marlène Mohier, Dominique Paturot, Dominic Richardson und Olivier Thévenon. Marc Pearson, Leiter der OECD-Abteilung Sozialpolitik, zeichnet für die ursprüngliche Konzeption der Berichte verantwortlich und hatte anschließend die Oberaufsicht. 


\section{Dieser Bericht enthält ...}

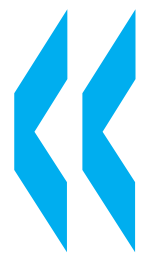

\section{StatLinks 部穴L}

Ein Service für OECD-Veröffentlichungen, der es ermöglicht, Dateien im Excel-Format herunterzuladen.

Suchen Sie die StatLinks rechts unter den in diesem Bericht wiedergegebenen Tabellen oder Abbildungen. Um die entsprechende Datei im Excel-Format herunterzuladen, genügt es,

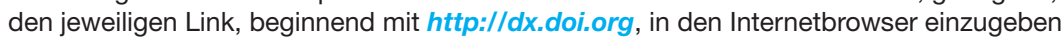
Wenn Sie die elektronische PDF-Version online lesen, dann brauchen Sie nur den Link anzuklicken. Sie finden StatLinks in weiteren OECD-Publikationen. 


\section{Inhaltsverzeichnis}

Kapitel 1. Soziale Schlüsselindikatoren $\ldots \ldots \ldots \ldots \ldots \ldots \ldots \ldots \ldots \ldots \ldots \ldots \ldots$

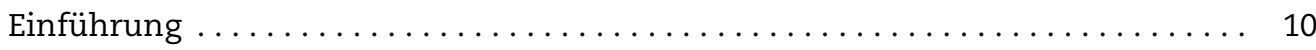

Soziale Schlüsselindikatoren in den OECD-Ländern $\ldots \ldots \ldots \ldots \ldots \ldots \ldots \ldots . \ldots$

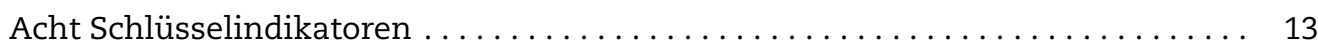

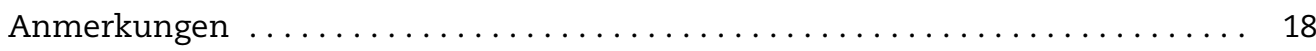

Kapitel 2. Schwerpunktthema: die Messung von Freizeit in den OECD-Ländern . . . . 19

Die Wirtschaftstheorie der Freizeit $\ldots \ldots \ldots \ldots \ldots \ldots \ldots \ldots \ldots \ldots \ldots \ldots \ldots . \ldots \ldots$

Trendmäßige Entwicklung der Restgröße der Erwerbsarbeitszeit . . . . . . . . 22

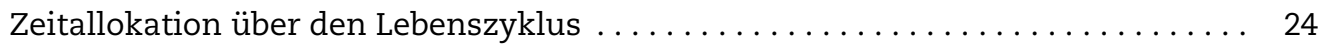

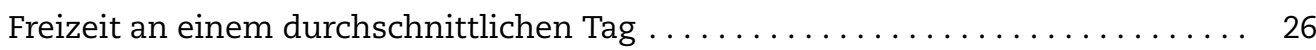

Entwicklungen im Zeitverlauf im Bereich Freizeit auf der Grundlage

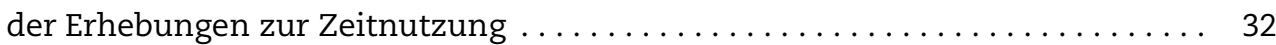

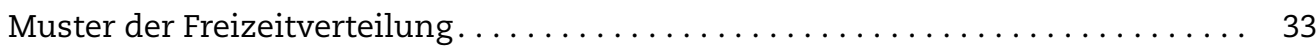

Arten von Freizeitaktivitäten . . . . . . . . . . . . . . . . . . . . . . 37

Zufriedenheit mit der Zeit, die auf die verschiedenen Aktivitäten

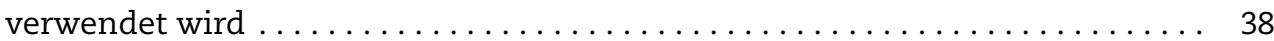

Freizeit im Vergleich zu Messgrößen der Lebenszufriedenheit und des Markteinkommens . . . . . . . . . . . . . . . . . . . . . . . . . . . . . . 40

Gesetzlicher Urlaubsanspruch und Freizeit . . . . . . . . . . . . . . . . 42

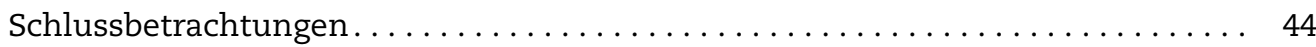

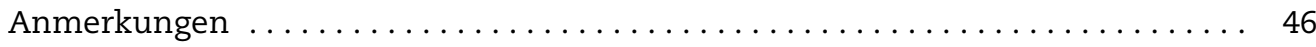

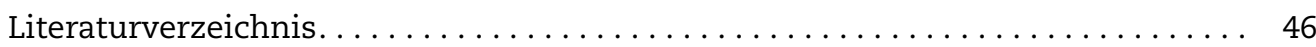

Anhang 2.A1.Wesentliche Merkmale der Erhebungen zur Zeitnutzung . . . . . . . 48

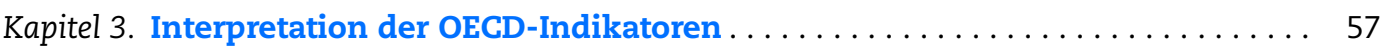

Der Zweck von Sozialindikatoren $\ldots \ldots \ldots \ldots \ldots \ldots \ldots \ldots \ldots \ldots \ldots \ldots . \ldots \ldots$

Der Rahmen der OECD-Sozialindikatoren . . . . . . . . . . . . . . . . . . . . 58

Auswahl und Beschreibung der Indikatoren . . . . . . . . . . . . . . . . . . 60

Was bietet diese Veröffentlichung. ............................ 66

Kapitel 4. Allgemeine Kontextindikatoren . . . . . . . . . . . . . . . . . . . . . . . 69

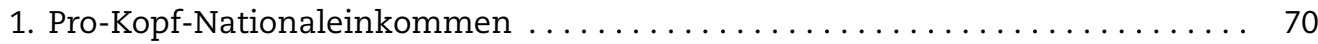

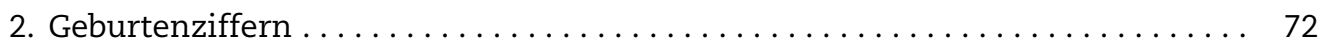

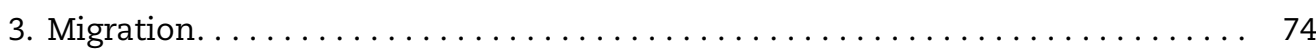

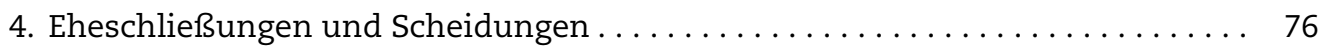




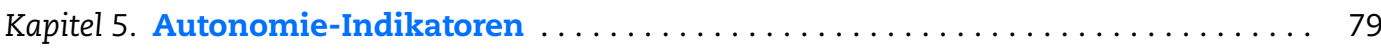

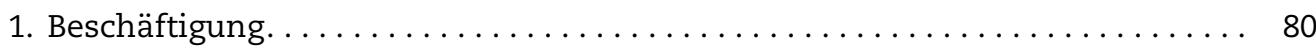

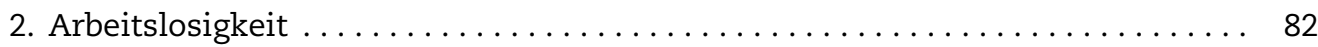

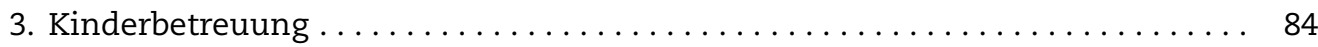

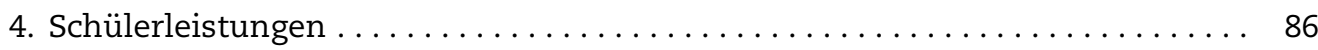

5. Nicht in Beschäftigung, Bildung oder Ausbildung . . . . . . . . . . 88

6. Alter bei Austritt aus dem Erwerbsleben . . . . . . . . . . . . . . . . . . 90

7. Bildungsausgaben ............................ 92

Kapitel 6. Indikatoren der sozialen Gerechtigkeit . . . . . . . . . . . . . . 95

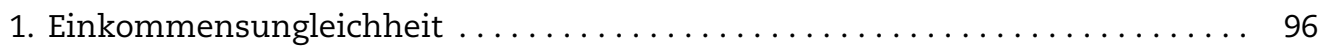

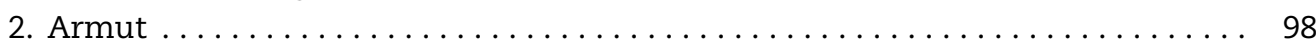

3. Kinderarmut. . . . . . . . . . . . . . . . . . . . . . . . . . . . . 100

4. Angemessenheit von Mindestsicherungsleistungen . . . . . . . . . . . . . 102

5. Öffentliche Sozialausgaben . . . . . . . . . . . . . . . . . . . . . . . . . . . . . . . . 104

6. Gesamtsozialausgaben .............................. 106

Kapitel 7. Gesundheitsindikatoren . . . . . . . . . . . . . . . . . . . . . 109

1. Lebenserwartung. . . . . . . . . . . . . . . . . . . . . . . . . . . . . . . . 110

2. Selbsteinschätzung des Gesundheitszustands . . . . . . . . . . . . . . 112

3. Gesundheitszustand von Säuglingen . . . . . . . . . . . . . . . . . . 114

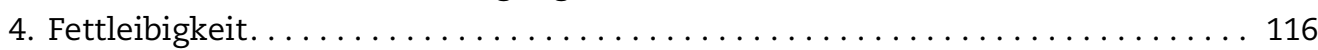

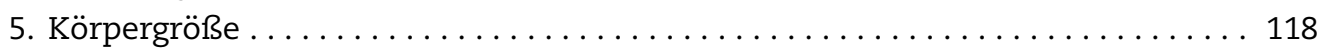

6. Seelische Gesundheit . . . . . . . . . . . . . . . . . . . . . . . . . . . 120

7. Langzeitpflegebedürftige . . . . . . . . . . . . . . . . . . . . . . . . . . . 122

8. Gesundheitsausgaben. . . . . . . . . . . . . . . . . . . . . . . 124

Kapitel 8. Indikatoren für den sozialen Zusammenhalt. . . . . . . . . . . . . . . . 127

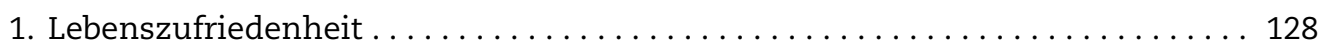

2. Arbeitszufriedenheit . . . . . . . . . . . . . . . . . . . . . . . . . 130

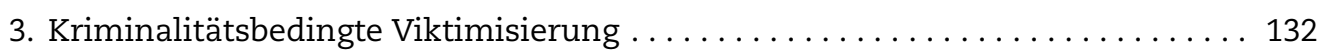

4. Suizide. . . . . . . . . . . . . . . . . . . . . . . . . . . . . . . . . . . . 134

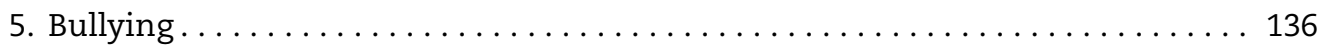

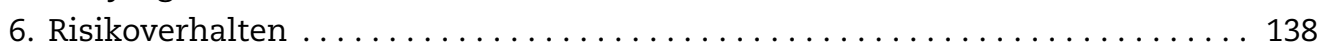




\section{Länderverzeichnis}

\begin{tabular}{|c|c|}
\hline AUS & Australien \\
\hline BEL & Belgien \\
\hline DNK & Dänemark \\
\hline DEU & Deutschland \\
\hline FIN & Finnland \\
\hline FRA & Frankreich \\
\hline GRC & Griechenland \\
\hline IRL & Irland \\
\hline ISL & Island \\
\hline ITA & Italien \\
\hline JPN & Japan \\
\hline CAN & Kanada \\
\hline KOR & Korea \\
\hline LUX & Luxemburg \\
\hline MEX & Mexiko \\
\hline NZL & Neuseeland \\
\hline NLD & Niederlande \\
\hline NOR & Norwegen \\
\hline AUT & Österreich \\
\hline POL & Polen \\
\hline PRT & Portugal \\
\hline SWE & Schweden \\
\hline CHE & Schweiz \\
\hline SVK & Slowakische Republik \\
\hline ESP & Spanien \\
\hline CZE & Tschechische Republik \\
\hline TUR & Türkei \\
\hline HUN & Ungarn \\
\hline USA & Vereinigte Staaten \\
\hline GBR & Vereinigtes Königreich \\
\hline
\end{tabular}



Kapitel 1

Soziale Schlüsselindikatoren 


\section{Einführung}

In den 1970er und 1980er Jahren wurden Sozialindikatoren entwickelt, um für die Beurteilung der Lebens- und Arbeitsbedingungen ein besseres Instrument zur Verfügung zu stellen, als die konventionellen Markteinkommensindikatoren. Heute liefern die verschiedenen Ausgaben von Gesellschaft auf einen Blick umfassende Informationen über die sozialen Bedingungen in verschiedenen OECD-Ländern und die zu ihrer Verbesserung ergriffenen Maßnahmen. Dieses reichhaltige Angebot hat aber seinen Preis. Für den Leser ist es schwierig, sich nach einem raschen Überfliegen von Gesellschaft auf einen Blick ein klares Bild davon zu machen, wie die sozialen Bedingungen im Ländervergleich aussehen und sich im Laufe der Zeit entwickeln.

Das Konzept der Schlüsselindikatoren ist eingeführt worden, um ein einfacheres Bild der sozialen Lage eines Landes zeichnen zu können. Diese Unterkategorie an Indikatoren erfüllt eine wichtige Kommunikationsfunktion, indem sie die Aufmerksamkeit der Leser rasch auf einige der kritischen Herausforderungen im sozialen Bereich lenkt, denen sich verschiedene OECD-Länder gegenübersehen, und komparative Fortschritte hervorhebt ${ }^{1}$.

In diesem Kapitel werden die Schlüsselindikatoren als fester Bestandteil von Gesellschaft auf einen Blick vorgestellt. Anschließend wird beschrieben, nach welcher Methode sie ausgewählt und zusammengesetzt wurden.

\section{Soziale Schlüsselindikatoren in den OECD-Ländern}

In Tabelle 1.1 und Tabelle 1.2 werden die acht ausgewählten Schlüsselindikatoren vorgestellt, jeweils zwei für jede der vier Dimensionen, in die Gesellschaft auf einen Blick gegliedert ist. Diese Tabellen ermöglichen es dem Leser, sowohl zu bestimmten Zeitpunkten (Tabelle 1.1) als auch in Bezug auf die sich im Zeitverlauf vollziehenden Veränderungen (Tabelle 1.2) sich einen raschen Überblick über die sozialen Bedingungen in den einzelnen Ländern zu verschaffen.

In Tabelle 1.1 werden die Leistungen jedes einzelnen OECD-Landes zu einem bestimmten Zeitpunkt (Tabelle 1.1) anhand von ampelähnlichen Symbolen gekennzeichnet. „Ampeln mit grünem Kreis“ werden für Länder verwendet, die in Bezug auf ihre Sozialleistungen in den drei oberen Dezilen angesiedelt sind, „Ampeln mit roter Raute“ für Länder in den drei unteren und „Ampeln mit gelbem Dreieck“ für Länder in den vier mittleren Dezilen.

In Tabelle 1.2 wird die Entwicklung der Sozialleistungen eines Landes im Vergleich zu anderen OECD-Ländern in jüngster Zeit anhand von „Richtungspfeilen“ beschrieben. „Nach oben zeigende grüne Pfeile“ stehen für Länder in den drei oberen Leistungsdezilen, „nach unten zeigende rote Pfeile“ für Länder in den drei unteren Leistungsdezilen und "nach rechts zeigende gelbe Pfeile“ für Länder, die auf Grund der Entwicklung ihrer Sozialleistungen in den vier mittleren Dezilen angesiedelt sind. 


\section{Tabelle 1.1. Soziale Schlüsselindikatoren für den jüngsten Zeitraum}

Diese Symbole beschreiben die Länderergebnisse zu einem bestimmten Zeitpunkt, wobei ein „grüner Kreis“ Länder in den drei oberen, eine „rote Raute“ Länder in den drei unteren und ein „gelbes Dreieck“ Länder in den vier mittleren Dezilen bezeichnet.

\begin{tabular}{|c|c|c|c|c|c|c|c|c|c|}
\hline & \multicolumn{2}{|c|}{ Autonomie } & \multicolumn{2}{|c|}{ Soziale Gerechtigkeit } & \multicolumn{2}{|c|}{ Gesundheit } & \multicolumn{2}{|c|}{ Sozialer Zusammenhalt } & \multirow{2}{*}{\begin{tabular}{|c} 
Einkommen \\
Pro-Kopf- \\
NNE zU \\
US-\$-KKP
\end{tabular}} \\
\hline & $\begin{array}{c}\text { Verhältnis } \\
\text { zwischen } \\
\text { Beschäftigung } \\
\text { und Bevölkerung, } \\
\text { insgesamt }\end{array}$ & $\begin{array}{c}\text { Anteil } \\
\text { der Schüler mit } \\
\text { unzureichenden } \\
\text { Lese- } \\
\text { kompetenzen }\end{array}$ & $\begin{array}{c}\quad \text { Gini- } \\
\text { Koeffizient der } \\
\text { Einkommens- } \\
\text { ungleichheit }\end{array}$ & $\begin{array}{l}\text { Geschlechts- } \\
\text { spezifisches } \\
\text { Lohngefälle }\end{array}$ & $\begin{array}{l}\text { Lebens- } \\
\text { erwartung } \\
\text { im Alter von } \\
65 \text { Jahren, } \\
\text { Männer }\end{array}$ & $\begin{array}{l}\text { Säuglings- } \\
\text { sterblichkeit }\end{array}$ & $\begin{array}{c}\text { Subjektives } \\
\text { Wohl- } \\
\text { befinden }\end{array}$ & $\begin{array}{c}\text { Kriminalitäts- } \\
\text { bedingte } \\
\text { Viktimi- } \\
\text { sierung }\end{array}$ & \\
\hline & $\begin{array}{l}\text { Niveau } \\
2007\end{array}$ & $\begin{array}{l}\text { Niveau } \\
2006\end{array}$ & $\begin{array}{c}\text { Niveau } \\
\text { 2004-2005 }\end{array}$ & $\begin{array}{c}\text { Niveau } \\
2006\end{array}$ & $\begin{array}{l}\text { Niveau } \\
2006\end{array}$ & $\begin{array}{l}\text { Niveau } \\
2006\end{array}$ & $\begin{array}{l}\text { Niveau } \\
2006\end{array}$ & $\begin{array}{l}\text { Niveau } \\
2005\end{array}$ & $\begin{array}{l}\text { Niveau } \\
2006\end{array}$ \\
\hline Australien & O & 0 & $\Delta$ & $\Delta$ & O & $\Delta$ & ○ & $\Delta$ & $\Delta$ \\
\hline Belgien & $\diamond$ & $\Delta$ & $\Delta$ & 0 & $\Delta$ & $\Delta$ & - & $\Delta$ & $\Delta$ \\
\hline Dänemark & O & 0 & ○ & O & $\diamond$ & $\Delta$ & O & $\bullet$ & O \\
\hline Deutschland & $\Delta$ & $\Delta$ & $\Delta$ & $\Delta$ & $\Delta$ & $\Delta$ & $\Delta$ & $\Delta$ & $\Delta$ \\
\hline Finnland & $\Delta$ & O & ○ & $\Delta$ & $\Delta$ & O & ○ & $\Delta$ & $\Delta$ \\
\hline Frankreich & $\Delta$ & $\Delta$ & $\Delta$ & ○ & O & $\Delta$ & $\Delta$ & ○ & $\Delta$ \\
\hline Griechenland & $\bullet$ & $\bullet$ & $\Delta$ & $\Delta$ & $\Delta$ & $\Delta$ & $\bullet$ & 0 & $\Delta$ \\
\hline Irland & $\Delta$ & 0 & $\bullet$ & $\Delta$ & $\Delta$ & $\Delta$ & $\diamond$ & $\bullet$ & O \\
\hline Island & ○ & $\Delta$ & $\Delta$ & $\Delta$ & 0 & O & $\Delta$ & $\diamond$ & $\Delta$ \\
\hline Italien & $\diamond$ & $\diamond$ & $\bullet$ & $\Delta$ & $\Delta$ & $\Delta$ & $\bullet$ & 0 & $\Delta$ \\
\hline Japan & $\Delta$ & $\Delta$ & $\Delta$ & $\bullet$ & 0 & O & $\Delta$ & 0 & $\Delta$ \\
\hline Kanada & O & 0 & $\Delta$ & $\Delta$ & 0 & $\diamond$ & ○ & $\Delta$ & - \\
\hline Korea & $\Delta$ & 0 & $\Delta$ & $\bullet$ & $\bullet$ & $\diamond$ & $\bullet$ & .. & $\diamond$ \\
\hline Luxemburg & $\bullet$ & $\diamond$ & ○ & O & $\Delta$ & ○ & $\Delta$ & $\Delta$ & 0 \\
\hline Mexiko & $\diamond$ & $\diamond$ & $\bullet$ & .. & $\Delta$ & $\bullet$ & $\Delta$ & $\bullet$ & $\bullet$ \\
\hline Neuseeland & ○ & ○ & $\bullet$ & 0 & 0 & $\diamond$ & O & $\diamond$ & $\diamond$ \\
\hline Niederlande & ○ & O & ○ & $\Delta$ & $\diamond$ & $\Delta$ & 0 & $\bullet$ & 0 \\
\hline Norwegen & ○ & $\Delta$ & $\Delta$ & O & 0 & O & 0 & $\Delta$ & ○ \\
\hline Österreich & $\Delta$ & $\Delta$ & ○ & $\Delta$ & $\Delta$ & 0 & $\Delta$ & 0 & $\Delta$ \\
\hline Polen & $\bullet$ & $\Delta$ & $\bullet$ & ○ & $\diamond$ & $\diamond$ & $\diamond$ & $\Delta$ & $\diamond$ \\
\hline Portugal & $\Delta$ & $\diamond$ & $\bullet$ & $\Delta$ & $\diamond$ & 0 & $\diamond$ & 0 & $\diamond$ \\
\hline Schweden & O & 0 & ○ & $\Delta$ & $\Delta$ & O & $\Delta$ & $\Delta$ & O \\
\hline Schweiz & - & $\Delta$ & - & $\Delta$ & 0 & $\Delta$ & ○ & $\bullet$ & 0 \\
\hline Slowak. Rep. & $\diamond$ & $\diamond$ & ○ & $\bullet$ & $\diamond$ & $\diamond$ & $\bullet$ & .. & $\diamond$ \\
\hline Spanien & $\Delta$ & $\diamond$ & $\Delta$ & $\Delta$ & 0 & $\Delta$ & $\Delta$ & 0 & $\Delta$ \\
\hline Tschech. Rep. & $\Delta$ & $\diamond$ & O & $\Delta$ & $\diamond$ & ○ & $\Delta$ & .. & $\diamond$ \\
\hline Türkei & $\diamond$ & $\diamond$ & $\bullet$ & .. & $\diamond$ & $\diamond$ & $\diamond$ & .. & $\diamond$ \\
\hline Ungarn & $\diamond$ & $\Delta$ & $\Delta$ & O & $\diamond$ & $\diamond$ & $\bullet$ & 0 & $\bullet$ \\
\hline Ver. Königreich & $\Delta$ & $\Delta$ & $\bullet$ & $\Delta$ & $\Delta$ & $\Delta$ & $\Delta$ & $\diamond$ & $\Delta$ \\
\hline Ver. Staaten & $\Delta$ & $\Delta$ & $\diamond$ & $\Delta$ & $\Delta$ & $\diamond$ & $\Delta$ & $\Delta$ & O \\
\hline
\end{tabular}

Anmerkung: NNE: Nettonationaleinkommen.

Auf Grund des qualitativen Charakters dieser Informationen sind in der Tabelle keine Indikatorwerte angegeben ${ }^{2}$. Als Kontextindikatoren enthalten die Tabellen (in der rechten Spalte) auch das Nettonationaleinkommen (NNE) in US-\$-Kaufkraftparitäten (KKP) (Tabelle 1.1) bzw. das reale Pro-Kopf-BIP-Wachstum (da nur für 17 Länder Angaben zum realen NNE-Wachstum zur Verfügung stehen) (Tabelle 1.2). 


\section{Tabelle 1.2. Relative Fortschritte bei sozialen Schlüsselindikatoren in jüngster Zeit}

Richtungspfeile beschreiben Leistungsveränderungen im Zeitverlauf, wobei „nach oben zeigende grüne Pfeile“ für Länder in den drei oberen, „nach unten zeigende rote Pfeile“ für Länder in den drei unteren und „nach rechts zeigende gelbe Pfeile" für Länder in den vier mittleren Leistungsdezilen stehen.

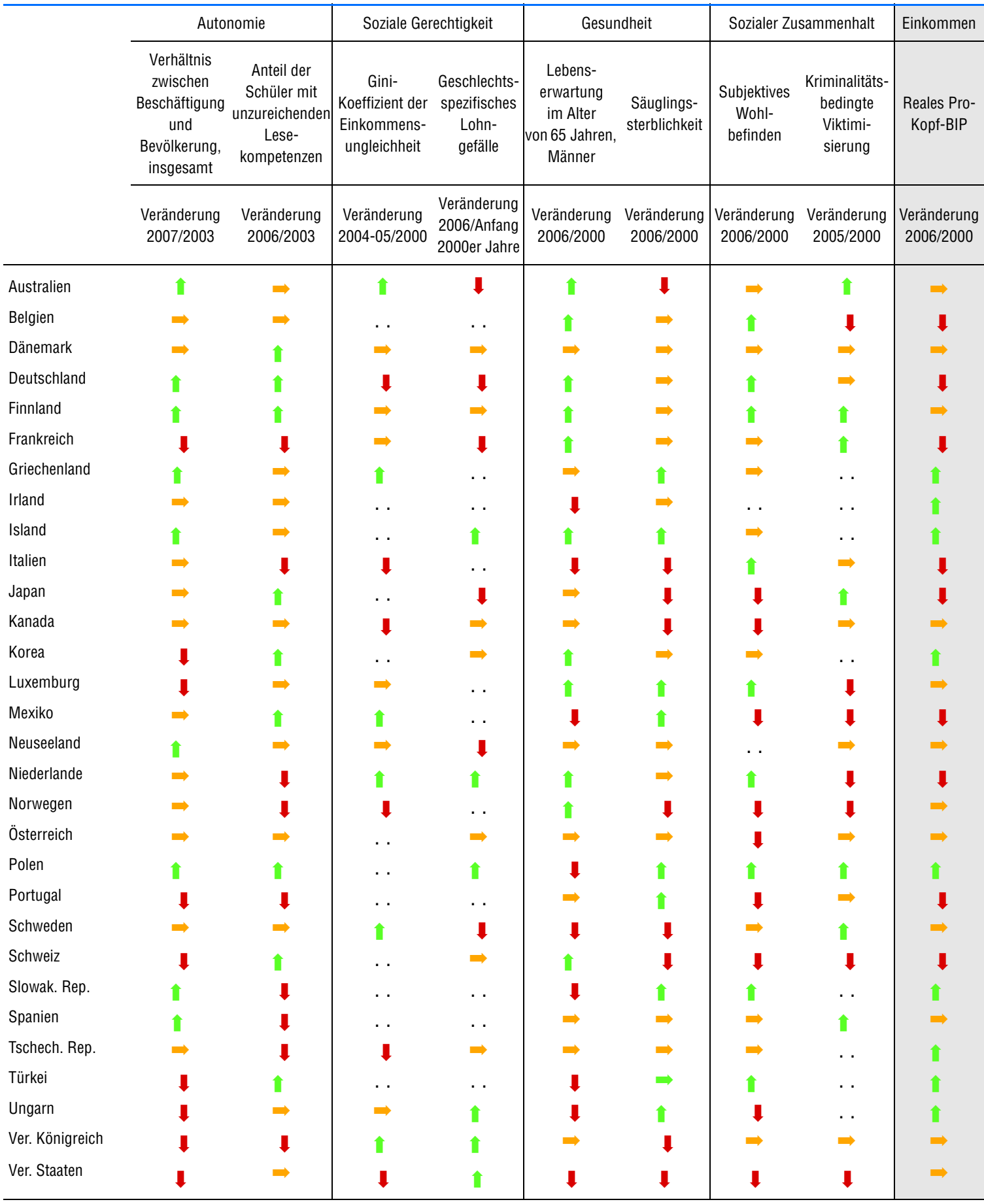

Anmerkung: Die Zeiträume für die Untersuchung der Veränderungen weichen auf Grund der unterschiedlichen Datenverfügbarkeit etwas voneinander ab. Die Veränderungen werden in arithmetischen Differenzen ausgedrückt, außer bei der kriminalitätsbedingten Viktimisierung (jahresdurchschnittliche Veränderungen). Einige Länderdaten zur kriminalitätsbedingten Viktimisierung gehen auf Daten vor dem Jahr 2000 zurück. Vgl. die Diskussion zu Indikator CO3 in Kapitel 8. Diese Fakten deuten darauf hin, dass die Vergleichbarkeit bei diesen Daten geringer ist als bei den Niveaudaten. 
Tabelle 1.1 zeigt eine Vielzahl von Entwicklungsmustern. Bei den meisten Ländern ist in Bezug auf die Leistungen das gesamte Ergebnisspektrum (grün, gelb und rot) zu beobachten. Nur Australien, Deutschland, Finnland, Frankreich, Norwegen, Österreich und Schweden haben keine „roten Ampeln“. Deutschland, Mexiko, die Türkei, das Vereinigte Königreich und die Vereinigten Staaten verzeichnen keine „grünen Ampeln“. Kein Land hat ausschließlich grüne Ampeln, doch weisen Dänemark, Neuseeland und Norwegen mit jeweils fünf die höchste Zahl an „grünen Ampeln“ auf.

Eine Untersuchung der Veränderungen bei den Indikatoren der sozialen Bedingungen ergibt ebenfalls ein uneinheitliches Bild. Wie in Tabelle 1.2 veranschaulicht wird, ist in den meisten Ländern bei den verschiedenen Indikatoren das gesamte Spektrum möglicher Veränderungen zu beobachten, wobei Dänemark, Finnland, Griechenland und Irland keine roten, Island, Kanada, Österreich und die Tschechische Republik keine grünen sowie Polen, die Slowakische Republik und die Türkei keine gelben Pfeile aufweisen. Polen zählt sechs grüne Pfeile, hat also in Bezug auf alle Schlüsselindikatoren, für die Daten aus diesem Land zur Verfügung stehen, große Fortschritte erzielt.

\section{Acht Schlüsselindikatoren}

Die sozialen Bedingungen anhand eines Katalogs von Schlüsselindikatoren in wenigen Worten zusammenzufassen, stellt eine große Herausforderung dar. In allen Fällen muss die Aufgabe folgende Kriterien erfüllen: a) die Leistungen beschreiben, b) über den breiten Katalog an Maßnahmen informieren, die innerhalb der vier Dimensionen der OECDTaxonomie der Sozialindikatoren vorgesehen sind (Autonomie, soziale Gerechtigkeit, Gesundheit und sozialer Zusammenhalt), c) die größtmögliche Zahl an OECD-Ländern erfassen und d) eine Beobachtung der Entwicklung des sozialen Status im Zeitverlauf ermöglichen.

Die Auswahl der Indikatoren kann auf einer statistischen Analyse fußen. Ein Konzept könnte beispielsweise darin bestehen, die Korrelationen zwischen allen in Gesellschaft auf einen Blick untersuchten Ergebnisindikatoren zu betrachten und innerhalb jedes Bereichs jene auszuwählen, die die höchste Korrelation untereinander aufweisen. Ein alternativer Ansatz wäre eine Art Faktorenanalyse, um innerhalb jedes Bereichs geeignete Kombinationen von Grundindikatoren zu identifizieren.

Nach der Auswahl der Indikatoren muss entschieden werden, wie diese am besten präsentiert werden. Die Indikatoren könnten „normalisiert“, d.h. zu einer 0-1-Skala standardisiert werden. Eine derartige Standardisierung erleichtert auch die Erstellung von Gesamtindizes für jeden der vier Bereiche der Sozialindikatoren oder eines globalen Index für alle vier Bereiche ${ }^{3}$. Jedoch bringt eine Standardisierung auch einen Verlust an Transparenz mit sich und erschwert dadurch die Kommunikation.

Die Erreichung der oben genannten Ziele wird auch durch Probleme bezüglich der Datenverfügbarkeit beeinträchtigt. Erstens sind auf Grund von Veränderungen bei den Definitionen und Quellen weniger Indikatoren aus Gesellschaft auf einen Blick über einen längeren Zeitraum in konsistenter Form verfügbar. Zweitens werden viele dieser Indikatoren nicht häufig bzw. noch nicht einmal in vorhersehbaren Intervallen aktualisiert und nur für eine begrenzte Zahl von Ländern erfasst. Schließlich richten viele dieser Indikatoren das Augenmerk auf ganz spezifische „Ergebnisse“ (z.B. Selbstmorde), deren Effekte bereits z.T. von anderen Indikatoren erfasst wurden (wie z.B. Lebenserwartung). 
In Anbetracht all dieser Einschränkungen erfolgt die Auswahl der Schlüsselindikatoren nicht nach statistischen Kriterien, sondern ist vielmehr das Resultat eines multinationalen Konsenses infolge von Konsultationsverfahren mit den Mitgliedsländern. Um ihre Aussagefunktion zu erfüllen, werden die ausgewählten Indikatoren in ihrem Rohformat dargestellt, ohne jegliche Standardisierung. Sie sind aber mit qualitativen Bezugsgrößen versehen, die die Interpretation der nummerischen Werte erleichtern. Um die gewünschte globale Darstellung zu erreichen, wurde die Zahl der Indikatoren für die einzelnen Dimensionen bewusst beschränkt.

Auf der Basis der oben genannten Erwägungen wurden für jede der vier Dimensionen der Sozialindikatoren (Autonomie, soziale Gerechtigkeit, Gesundheit und sozialer Zusammenhalt) zwei Schlüsselindikatoren ausgewählt. Die so erhaltenen acht Schlüsselindikatoren ermöglichen einen internationalen Vergleich der Sozialbedingungen in den einzelnen Ländern und eine Verfolgung ihrer Entwicklung im Zeitverlauf. Die der Auswahl der Schlüsselindikatoren zu Grunde liegenden qualitativen Erwägungen werden weiter unten beschrieben.

\section{Autonomie}

Die Hauptbestimmungsfaktoren der Autonomie einer Person sind der Zugang zu Beschäftigung und die Qualifikationen. Was die Arbeit betrifft, so ist die Beschäftigungsquote der Bevölkerung im erwerbsfähigen Alter ein geeigneter Indikator für die Situation einer Person am Arbeitsmarkt. Dieser auf vergleichbaren Definitionen aus Arbeitskräfteerhebungen basierende Indikator ist für alle OECD-Länder im Jahresrhythmus verfügbar. Im Vergleich zu anderen Messgrößen des Arbeitskräfteüberhangs am Arbeitsmarkt, wie die Arbeitslosenquote, wird die Beschäftigungsquote von den Entscheidungen des Einzelnen, sich bei ungünstigen Beschäftigungsaussichten vom Arbeitsmarkt zurückzuziehen, weniger stark beeinflusst.

Die umfassendste derzeit verfügbare Messgröße der Qualifikationen und Kompetenzen der Bevölkerung ist die durchschnittliche Schulbesuchsdauer der Bevölkerung im Erwerbsalter. Jedoch trägt dieser Indikator weder der außerhalb der Schulen angebotenen Humankapitalbildung noch der Qualität der Schulausbildung gebührend Rechnung 4 . In dem Maße, wie die Qualifikationen der Personen im Erwerbsalter Auswirkungen auf ihre Arbeitsmarktergebnisse haben, werden diese Qualifikationen z.T. in den Beschäftigungsquoten mit erfasst. Es sprechen insofern gute Gründe dafür, den Schwerpunkt auf die Kompetenzen der Menschen vor dem Eintritt ins Erwerbsleben zu legen, als diese vorberuflich erworbenen Qualifikationen Hinweise auf die künftigen Arbeitsmarktaussichten und Lebenschancen liefern. Messgrößen dieser Kompetenzen für 15-Jährige werden im Rahmen der Internationalen Schulleistungsstudie der OECD (PISA) zur Verfügung gestellt. Diese Messgrößen basieren auf vergleichbaren Erhebungsmodulen, die alle drei Jahre erneut eingesetzt werden und alle OECD-Länder umfassen. Zu Grunde gelegt wird der Indikator des Anteils der 15-jährigen Schülerinnen und Schüler mit Lesekompetenzen auf oder unter Stufe 1, d.h. unter dem zur Ausübung normaler Aktivitäten im Alltag notwendigen Mindestniveau. Im Vergleich zu anderen (PISA-)Messgrößen der Schülerkompetenzen hat dieser Indikator den Vorteil, dass er sich auf jene jungen Menschen konzentriert, die, einmal erwachsen, häufiger arbeitslos, in einem Niedriglohnjob gefangen oder von Sozialhilfe abhängig sind. 


\section{Soziale Gerechtigkeit}

Die soziale Gerechtigkeit ist ein Konzept, das für ein breites Spektrum an Sozialergebnissen, wie Einkommen, Gesundheit und Bildung, relevant ist. Allerdings sind nur wenige der derzeit verfügbaren Indikatoren der sozialen Gerechtigkeit in einem breiten Kreis von Ländern untersucht worden, bieten eine gute Vergleichbarkeit und werden in regelmäßigen Intervallen erhoben. Die beiden ausgewählten Indikatoren konzentrieren sich auf die Einkommensungleichheit und das Lohngefälle zwischen Männern und Frauen.

Einkommensdifferenzen zwischen den Einwohnern eines Landes sind ein offensichtlicher Ausdruck für Unterschiede bei den Lebensbedingungen. Werden diese Unterschiede zu groß, können sie dem von allen geteilten Grundsatz der sozialen Gerechtigkeit zuwiderlaufen. Daten zur Verteilung des verfügbaren Einkommens der privaten Haushalte werden von der OECD alle fünf Jahre zusammengetragen. Diese Daten ermöglichen es, die Einkommensungleichheit in allen OECD-Ländern zu vergleichen und ihre Entwicklung (seit dem Jahr 2000) in etwa zwei Dritteln der Länder zu verfolgen. Als Indikator dient der Gini-Koeffizient des verfügbaren Haushaltseinkommens. Dieser Indikator bietet eine gute Synthese der Gesamtverteilung des Haushaltseinkommens und ist mit den Messgrößen der relativen Armut angemessen korreliert. Keine perfekte Korrelation besteht hingegen zwischen dem Gini-Koeffizienten und seinen Veränderungen und anderen Messgrößen der Einkommensungleichheit, wie der Armut, auf Länderebene (Norwegen beispielsweise weicht von der Korrelationskurve zwischen Veränderungen des GiniKoeffizienten und der Armut stark ab). So kann die Auswahl des Gini-Koeffizienten gegenüber anderen Messgrößen der Ungleichheit das Länderranking bei den Schlüsselindikatoren beeinflussen. Außerdem sind Gini-Koeffizienten intuitiv nicht zu verstehen.

Die Indikatoren der Einkommensungleichheit fußen auf der Annahme, dass alle Mitglieder eines Haushalts die verfügbaren Ressourcen teilen. Von ihrem Aufbau her eignen sich diese Messgrößen daher nicht für eine Beurteilung der zwischen Männern und Frauen bestehenden Unterschiede, die in allen OECD-Ländern eine wichtige Dimension der sozialen Gerechtigkeit darstellen. Diese geschlechtsspezifischen Unterschiede werden anhand des Verhältnisses zwischen dem Medianverdienst vollzeitbeschäftigter Frauen und Männer beschrieben. Dieser Indikator trägt zwar nur einem Teil der Nachteile Rechnung, denen Frauen am Arbeitsmarkt begegnen, lässt sich aber auf Länderebene leichter vergleichen und wird in regelmäßigen Intervallen berechnet. Unberücksichtigt bleibt indessen der durch die Aufteilung des Familieneinkommens zwischen Männern und Frauen entstehende Glättungseffekt. Die OECD Earnings Database enthält für 19 OECDLänder Angaben zum Lohngefälle zwischen den Geschlechtern.

\section{Gesundheitszustand}

Die beiden Hauptbestimmungsfaktoren des Gesundheitszustands sind Mortalität und Morbidität. Leider existiert gegenwärtig keine vollständige, auf regelmäßiger Basis verfügbare Messgröße der Morbidität ${ }^{5}$. Aus diesem Grund betreffen die beiden verwendeten Indikatoren das Mortalitätsrisiko der Personen an beiden Extremen des Altersspektrums.

Für ältere Menschen wird der Indikator der Lebenserwartung im Alter von 65 Jahren verwendet, zu dem Informationen in den OECD-Gesundheitsdaten verfügbar sind. Dieser weist zwei geringfügige Nachteile auf: Keine Datenreihe deckt derzeit die gesamte 


\section{Kasten 1.1. Messung der Veränderungen der Lebenszufriedenheit}

Die Gallup World Poll von 2006, auf der die Untersuchung des Niveaus der Lebenszufriedenheit in dieser Ausgabe 2009 von Gesellschaft auf einen Blick beruht, liefert keine Zeitreihendaten zur Lebenszufriedenheit. So wurden die Daten zur Entwicklung der Lebenszufriedenheit aus anderen Quellen zusammengestellt. Die Ausgangsquelle war eine aus der World Database of Happiness (WDH) (vgl. www1.eur.nl/fsw/happiness/hap_nat/ nat_fp.htm) zusammengetragene Datensammlung. Die Daten wurden der WDH-Website am 3. und 4. Oktober 2008 entnommen.

Veränderungen der Lebenszufriedenheit wurden über den Zeitraum 20002006 untersucht. Der Zeitraum wurde als hinreichend lang eingestuft, um Veränderungen zu Tage treten zu lassen. Außerdem fiel das festgelegte Enddatum mit dem Datum der Gallup-Erhebung zusammen.

Daten zu Veränderungen der Lebenszufriedenheit standen für 28 OECD-Länder zur Verfügung. Von den Länder-Zeitreihendaten der WDH waren 20 der EurobarometerUmfrage entnommen. Mit Ausnahme der Türkei waren alle Länder europäische Länder. Die Daten für das Vereinigte Königreich bezogen sich allein auf Großbritannien. Für weitere vier Länder stammten die Daten ursprünglich aus dem World Values Survey (Kanada, Korea, Mexiko und die Vereinigten Staaten). Die Daten für Norwegen und die Schweiz waren der Europäischen Sozialerhebung (European Social Survey) entnommen. Die Daten für Australien stammten aus dem Australian Unity Wellbeing Index und die Daten für Japan aus der Life in Nation-Erhebung. Für Island und Neuseeland standen keine Daten zur Verfügung.

Die ursprüngliche Skala der Lebenszufriedenheit der Eurobarometer-Umfrage reichte, genauso wie die der japanischen Erhebung, von 1-4. Zur Anpassung an eine Skala von 0-10 wurde das WDH-Transformationssystem verwendet. Der ursprüngliche World Values Survey hatte eine Skalierung von 1-10; zur Anpassung dieser Daten kam ebenfalls das Transformationssystem der WDH zum Einsatz. Die Daten für Australien und Norwegen mussten nicht konvertiert werden.

Die ins Englische übersetzten Fragen wichen in den einzelnen Erhebungen leicht voneinander ab. Ins Deutsche übertragen lauteten sie in der Eurobarometer-Umfrage wie folgt: „Wie zufrieden sind Sie mit dem Leben, das Sie führen?“, während im World Values Survey folgende Frage gestellt wurde: „Wie zufrieden sind Sie in diesem präzisen Moment mit Ihrem Leben insgesamt?“ In der australischen Erhebung lautete die Frage: „Wie zufrieden oder unzufrieden sind Sie zurzeit mit Ihrem Leben insgesamt?" In den Erhebungen für Kanada, die Schweiz und Norwegen wurde die WVS-Formulierung übernommen, in der japanischen Erhebung die Frage aus der Eurobarometer-Umfrage.

Notgedrungen bezogen sich die Veränderungsdaten für Polen, die Slowakische Republik, die Tschechische Republik, die Türkei und Ungarn auf den Zeitraum 2001-2006. Auch die Daten für Mexiko erstreckten sich auf einen Fünfjahreszeitraum, 2000-2005, die Daten für Japan betrafen den Zeitraum 2001-2007. Die Daten für Korea deckten einen Vierjahreszeitraum ab, 2001-2005, die norwegischen Daten den Zeitraum 2002-2006 und die Schweizer Daten den Zeitraum 2002/2003-2006/2007. Die Daten für die Vereinigten Staaten bezogen sich auf die Jahre 1999-2006. Für die übrigen insgesamt 17 Länder standen die Daten für den gewünschten Zeitraum 2000-2006 zur Verfügung. 


\section{Kasten 1.1. Messung der Veränderungen der Lebenszufriedenheit (Forts.)}

Saisonale Effekte waren eine weitere Einschränkung. Laut WDH wurden die Eurobarometer-Daten größtenteils im April oder April-Mai 2000 und im Frühjahr 2006 erhoben, d.h. in etwa in derselben Jahreszeit. Die Eurobarometer-Daten für die vier Länder, für die die Daten den Zeitraum 2001-2006 abdeckten, wurden jeweils im Oktober 2001 und Frühjahr 2006 erhoben. Für Australien stammten die Daten von September 2000 und Oktober 2006. Die kanadischen Daten waren von August 2000 und einem unbestimmten Zeitraum im Jahr 2006. In den japanischen Daten wurden die Monate September 2000 und Juli 2006 gegenübergestellt. Die Daten für die Schweiz wurden zwischen September 2002 und Februar 2003 sowie August 2006 und April 2007 erhoben. Die koreanischen Daten für das Jahr 2001 waren von November. Über das genaue Erhebungsdatum der zweiten Datenreihe für Korea von 2005 liegen keine Angaben vor. Die norwegischen Daten wurden im September 2002 und im Zeitraum August-Dezember 2006 gewonnen.

Trotz dieser Uneinheitlichkeiten wurde die Auffassung vertreten, dass sich die aus den oben genannten Kompromissen resultierende Minderung der durchschnittlichen Datenqualität durch die Vorteile rechtfertigt, die aus der Aufnahme einer größeren Zahl von Länderbeobachtungen in die Daten resultieren. Allerdings darf der Leser die Datenqualitätsprobleme nicht aus den Augen verlieren, und bei der Interpretation der detaillierten Länderrankingdaten der Veränderungen der Lebenszufriedenheit ist entsprechend Vorsicht geboten.

Die Korrelation zwischen den zur Berechnung von Veränderungen der Lebenszufriedenheit verwendeten Endpunktdaten (weitgehend aus dem Jahr 2006, aber auch den Jahren 2005 und 2007) und den Daten der Gallup Poll war recht stark ( $r=0,81)$. Die größten Ausreißer waren die Türkei und Mexiko.

Die aus den Daten hervorgehende stärkste Veränderung betrifft den in der Eurobarometer-Umfrage registrierten sehr starken Anstieg der Lebenszufriedenheit in der Türkei von 4,6 im Jahr 2000 auf 6,1 im Jahr 2006. Eine zweite türkische Erhebung (mittels des World Values Survey) ergab unter Verwendung einer 10-Stufen-Zufriedenheitsskala einen parallelen, ja sogar noch größeren Anstieg von 5,6 im Jahr 2000 auf 7,5 im Jahr 2007. In der Eurobarometer-Umfrage von 2006 rangiert die Türkei vor Griechenland, Portugal, der Slowakischen Republik und Ungarn. Demgegenüber ergeben die Daten der Gallup World Poll für die Türkei 2006 den niedrigsten Zufriedenheitsgrad im OECD-Raum.

Nahezu alle hier zu Grunde gelegten Erhebungen bezogen sich auf einen kleinen Stichprobenumfang (mehrere Tausend Befragte). Nicht klar ist, welcher Anteil der erfassten Veränderungen der Lebenszufriedenheit statistisch signifikant ist.

Altenbevölkerung ab, und in einigen Ländern werden die Datenreihen nicht alljährlich aktualisiert ${ }^{6}$. Für Kinder wird als Indikator die Säuglingssterberate zu Grunde gelegt, d.h. die Zahl der Todesfälle bei Kindern unter einem Lebensjahr, je 1000 Lebendgeburten. Ein potenzielles Problem, das in Verbindung mit den Säuglingssterberaten besteht, ist die von Land zu Land unterschiedliche statistische Erfassung von Frühgeburten, obwohl es möglich ist, dass die Auswirkungen dieses Problems auf die Datenvergleichbarkeit überzeichnet werden (vgl. die Diskussionen zu Indikator HE3.1 in Kapitel 7).

\section{Sozialer Zusammenhalt}

Der soziale Zusammenhalt hat positive und negative Dimensionen. Auf der positiven Seite gibt der Indikator Aufschluss über die Teilnahme der Bürger am Gemeinschaftsleben 
und ihre Einstellung anderen gegenüber. Auf der negativen Seite können verschiedene Pathologien, wie Suizid, Risikoverhalten oder Kriminalität, auf einen mangelnden sozialen Zusammenhalt hindeuten.

Keine Messgröße erfasst die Teilnahme der Bevölkerung am Gemeinschaftsleben bzw. ihre Einstellungen anderen gegenüber in ihrer Gesamtheit. Dennoch zeigen Forschungsergebnisse, dass mehrere dieser Merkmale - zusammen mit persönlichen Eigenschaften - zur Lebenszufriedenheit der Menschen beitragen. Aus diesem Grund wird zur Messung der positiven Dimensionen des sozialen Zusammenhalts in verschiedenen Ländern die durchschnittliche Lebenszufriedenheit als Indikator herangezogen. Der Grad der Lebenszufriedenheit wird anhand der Punktzahl der Länder im Gallup World Poll von 2006 bestimmt. Die Daten zur Messung von Veränderungen der Lebenszufriedenheit stammen aus einer Vielzahl von Quellen (vgl. Kasten 1.1). Zwar ist nicht von der Hand zu weisen, dass dieser Indikator die Beteiligung des Einzelnen am Leben der Gemeinschaft und die Einstellung der Bürger zueinander nur indirekt misst, doch sind die anderen verfügbaren Indikatoren noch weniger intuitiv.

Zur Messung der negativen Ausdrucksformen des sozialen Zusammenhalts wurde der Indikator des Niveaus der kriminalitätsbedingten Viktimisierung ausgewählt, d.h. der Anteil der Personen, die im vorangegangenen Kalenderjahr Opfer von Straftaten waren. Die Daten sind dem International Crime Victim Survey entnommen, dessen jüngste Erhebungswelle 26 OECD-Länder umfasste. Der hier verwendete Indikator bezieht sich auf die zehn Kategorien von Vergehen, die Gegenstand aller Erhebungswellen sind.

\section{Anmerkungen}

1. Schlüsselindikatoren werden auch in anderen OECD-Arbeitsbereichen verwendet. So enthält z.B. der OECD-Bericht Environment at a Glance - OECD Environmental Indicators einen Katalog von (10) „Umwelt-Schlüsselindikatoren“ die von den Ministern der OECD-Länder als Informations- und Kommunikationsinstrument für die Öffentlichkeit gebilligt wurden.

2. Leser, die an den nummerischen Werten der Schlüsselindikatoren interessiert sind, werden auf die nachstehenden Kapitel zu den einzelnen Indikatoren und die diesbezüglichen OECD-Webseiten (www.oecd.org/els/social/indicators) verwiesen.

3. Ein Beispiel für einen auf der Basis von 16 OECD-Sozialindikatoren erstellten „Gesamtindex" findet sich in Kapitel 2 von Gesellschaft auf einen Blick 2006.

4. Zur Messung dieser Kompetenzen bedarf es Erhebungen, die die gesamte Bevölkerung erfassen und deren Daten für die meisten OECD-Länder zur Verfügung stehen. An der 2003 von der OECD durchgeführten Internationalen Erhebung der Lesefähigkeit und der Lebenskompetenzen Erwachsener (ALLS) waren nur fünf OECD-Länder beteiligt. Das OECD-Programm für die Internationale Beurteilung der Kompetenzen Erwachsener (PIAAC - Programme for the International Assessment of Adult Competences) wird künftig einen Kompetenzindikator für die gesamte Erwachsenenbevölkerung zur Verfügung stellen.

5. Selbstangaben der Betroffenen zu ihrem Gesundheitszustand sind eine mögliche Messgröße der Morbidität. Die OECD-Gesundheitsdaten enthalten Informationen zu dieser Variable, ermöglichen aber keine Vergleiche in hinreichend regelmäßigen Intervallen.

6. Aus diesem Grund bezieht sich der in den Tabelle 1.1 und 1.2 dargelegte Indikator ausschließlich auf die ältere männliche Bevölkerung. Es hätte genauso gut ein Indikator der Lebenserwartung älterer Frauen dargestellt oder eine Messgröße konstruiert werden können, die die Lebenserwartung der Männer und Frauen kombiniert. 
Kapitel 2

\section{Schwerpunktthema: Die Messung von Freizeit in den OECD-Ländern}


$\mathrm{U}$ mfang und Qualität von Freizeit sind auf Grund der unmittelbaren Zufriedenheit, die daraus resultiert, für das Wohlergehen der Menschen von Bedeutung. Darüber hinaus ist auf eine bestimmte Weise verbrachte Freizeit wichtig für die körperliche und seelische Gesundheit. Freizeit trägt ferner auch zum Wohlergehen der Menschen im Umfeld der unmittelbar Freizeit genießenden Person bei. Wenn eine Person Freizeitaktivitäten nachgeht, teilt sie den daraus gewonnenen Nutzen auf vielfältige Weise mit anderen, beispielsweise durch die Verbesserung persönlicher Beziehungen sowie des Zusammenlebens innerhalb der Familie und durch die Schaffung von Sozialkapital in Form von Netzwerken (wenigstens bei bestimmten Formen gemeinsam verbrachter Freizeit). Die Freizeitmuster in den OECD-Ländern verdienen deshalb als wichtiger Teil des Sozialmonitoring Berücksichtigung in den Erhebungen.

Was genau ist also Freizeit? Freizeit kann im Hinblick auf Zeit, Aktivitäten oder Gemütszustände definiert werden. In Bezug auf die Zeit kann Freizeit als jene Zeit betrachtet werden, die frei von Verpflichtungen und Notwendigkeiten verbracht wird. In quantitativer Hinsicht ist Freizeit z.B. definiert worden als „alle Aktivitäten, die nicht von jemand anders gegen Bezahlung für uns übernommen werden können und die wir effektiv nicht zu tun brauchen, wenn wir nicht möchten“ (Burda et al., 2007, S. 1). Trotz ihrer Vorzüge geht diese Definition nicht explizit auf die Art von Aktivitäten ein, die als Freizeit betrachtet werden können. Sie beschreibt auch nicht, bis zu welchem Grad eine Person frei von Verpflichtungen ist. Alternativ kann Freizeit als bestimmte Aktivitäten definiert werden, die für gewöhnlich als Freizeitaktivitäten betrachtet werden. Eine umfassendere Definition könnte auf dem beruhen, was die meisten Menschen als Freizeitaktivitäten anführen würden, etwa Fernsehen, Aktivitäten im Sport- bzw. Fitnessbereich, Lesen, Kinobesuche usw. Letztlich kann Freizeit auch als Gemütszustand definiert werden, d.h. als Praktizieren angenehmer bzw. vergnüglicher Beschäftigungen. Die hier tatsächlich verwendeten Messgrößen der Freizeit beruhen auf all diesen Definitionen.

Dieses Kapitel bietet zunächst eine kurze Analyse der einschlägigen Fachliteratur über die ökonomischen Bestimmungsfaktoren der Freizeit. Anschließend wird die Freizeit im OECD-Raum als nicht mit Erwerbsarbeit verbrachte restliche Zeit untersucht. Dieser Restzeit-Ansatz in Bezug auf die Daten ist nicht ideal, weil er insbesondere keine Veränderung des Umfangs der geleisteten unentgeltlichen Arbeit im Länder- oder Zeitvergleich ermöglicht. Indessen ermöglicht der Restzeit-Ansatz die Betrachtung der Freizeit für die größtmöglichen Länderquerschnitte und -zeitreihen im OECD-Raum. Im Anschluss daran erfolgt eine weitere Kontextualisierung der Freizeit unter Betrachtung der sehr allgemeinen Allokation von Zeit über den Lebenszyklus erwachsener Menschen. Diese Kontextualisierung erfolgt im Durchschnitt der OECD-Länder in Bezug auf die Jahre vor der Schulpflicht, die Schulzeit, die Jahre vor Eintritt in den Arbeitsmarkt, die nicht mit Erwerbsarbeit verbrachten Jahre, die mit Erwerbsarbeit verbrachten Jahre und die im Ruhestand verbrachten Jahre, aufgeschlüsselt nach Männern und Frauen. 
Für die kleinere Untergruppe der 18 OECD-Länder, für die vergleichbare Daten beschafft werden konnten, werden Studien zur Zeitnutzung verwendet, um die Freizeit an einem typischen Tag und im Zeitverlauf genauer zu untersuchen (im Anhang zu diesem Kapitel finden sich nähere Angaben zur Vergleichbarkeit der 18 verwendeten Erhebungen zur Zeitnutzung). In diesen Erhebungen wird die Zeit, die der Erwerbstätigkeit bzw. sonstigen Aktivitäten gewidmet wird, exakt gemessen, indem Daten zur Zeitallokation der Befragten im Rahmen oder außerhalb ihrer beruflichen Tätigkeit erfasst werden. Die Beschreibungen der Aktivitäten durch die Befragten werden allgemeinen Kategorien entsprechend kodiert, etwa „mit Erwerbsarbeit verbrachte Zeit“, „mit Haushaltsarbeit verbrachte Zeit“ oder „mit Freizeitaktivitäten verbrachte Zeit“. Die Methoden und Ansätze variieren zwar bis zu einem gewissen Grad, in allen in diesem Kapitel verwendeten Erhebungen zur Zeitnutzung ist die Kategorie „Freizeit“ jedoch als die Summe nicht zwingend erforderlicher Aktivitäten wie Hobbys, Fernsehen oder Radiohören, soziale Begegnungen mit Freunden und Familie, Besuch kultureller Veranstaltungen, Ausrichtung von Ereignissen sowie Ausübung sportlicher Aktivitäten definiert. Alle Erhebungen zielen darauf ab, genau zu messen, was die Menschen mit ihrer Zeit effektiv anfangen, nicht, was sie ihrer Erinnerung zufolge lange nach den Ereignissen damit getan haben. Nach Bereinigung können Freizeitniveaus und -trends anhand dieser Daten länderübergreifend verglichen werden. Das Augenmerk des zweiten Teils dieses Kapitels liegt - nach wie vor unter Verwendung der Daten zur Zeitnutzung - auf den Mustern der Freizeitverteilung nach Geschlecht und Alter ${ }^{1}$. In diesem Teil des Kapitels wird auch auf die Arten von Freizeitaktivitäten eingegangen, denen die Menschen nachgehen, und den Grad der Zufriedenheit, die sie nach deren Ausführung empfinden. Abschließend wird die Beziehung zwischen Freizeit und anderen Messgrößen des Wohlergehens sowie Freizeit und Politikentscheidungen zu bezahlten Urlaubstagen erörtert.

\section{Die Wirtschaftstheorie der Freizeit}

Seit Veblens Theorie der feinen Leute (Theory of the Leisure Class) aus dem ausgehenden 19. Jahrhundert haben sich Volkswirte und andere Sozialwissenschaftler intensiv für Freizeit interessiert. Das Hauptaugenmerk der meisten Arbeiten zum Arbeitsangebot in der neoklassischen Tradition liegt auf der theoretischen und empirischen Forschung zur Wahl zwischen Arbeit und Freizeit. Bei diesem Ansatz werden im Allgemeinen jedoch andere Formen der Zeitnutzung ignoriert. Effektiv wird damit die Differenz zwischen Erwerbsarbeit und allen anderen Formen der Zeitnutzung insgesamt („Restzeit“) untersucht, was entsprechend den Methoden der Optimierung unter Nebenbedingungen der neoklassischen Wirtschaftstheorie Freizeit natürlich als Untergruppe enthält (vgl. Caussa, 2008, wegen der jüngsten OECD-Arbeiten in dieser Richtung).

Der kanonische moderne Ansatz im Hinblick auf die Zeitnutzung, bei dem Freizeit auf komplexere Art und Weise analysiert wird, ist Gronau (1976) zuzurechnen. Auf der Grundlage der früheren Arbeiten von Mincer argumentiert Gronau für die Notwendigkeit, zwischen unentgeltlicher Arbeit (Produktion für den Eigenverbrauch) und Freizeit zu unterscheiden. Er macht geltend, dass die Begründung für die ausschließliche Konzentration auf die Wahl zwischen Erwerbsarbeit und Restzeit - eine Konzentration, die er ablehnt - sich auf eine angenommene Stabilität der Allokation der Restzeit auf konkurrierende Nutzungsmöglichkeiten (z.B. Produktion für den Eigenverbrauch, Freizeit und Schlaf) in Reaktion auf wirtschaftliche Veränderungen stützt. Gronau entwickelt ein formales Modell mit einer dreifachen Unterscheidung zwischen Freizeit, Produktion für 
den Eigenverbrauch und Erwerbsarbeit. Sein Modell beruht auf der Annahme, dass handelbare Güter, die durch Erwerbsarbeit erlangt wurden, und für den Eigenverbrauch produzierte Güter perfekte Substitute sind. Ein Anstieg der Marktlöhne verringert die Produktion für den Eigenverbrauch. Die Auswirkungen der Löhne auf Freizeit wie auf Erwerbstätigkeit sind unbestimmt. Ein Einkommensanstieg führt zu mehr Freizeit, weniger Erwerbsarbeit und unveränderter Produktion für den Eigenverbrauch. Die empirischen Forschungsarbeiten von Bloch und Gronau anhand von Daten aus den Vereinigten Staaten und Israel lassen darauf schließen, dass Freizeit bei Paaren positiv mit dem Einkommen des Ehemanns zusammenhängt, negativ mit dem Einkommen der Ehefrau und wiederum positiv mit dem Nichtlohneinkommen. Darüber hinaus wirkt sich eine größere Anzahl von Kindern - insbesondere im Vorschulalter - freizeitverringernd aus (Gronau, 1976, Tabelle 1).

Andere Erweiterungen von Arbeitsangebotsmodellen, die die Produktion für den Eigenverbrauch mitberücksichtigen, finden sich u.a. bei Chiappori (1997) sowie Apps und Rees (1996, 1997 und 2002). Zusätzlich zu Erwerbstätigkeit, Produktion für den Eigenverbrauch und Freizeit wurde Gronaus Modell von Solberg und Wong (1992) auch um Zeiten für das Berufspendeln erweitert. Die empirischen Befunde der Autoren stimmen jedoch nicht mit den Vorhersagen ihres Modells überein, was sie hauptsächlich auf die Verletzung ihrer (mit Gronau geteilten) Annahme der perfekten Substituierbarkeit zwischen Erwerbsarbeit und Produktion für den Eigenverbrauch zurückführen.

In keinem der vorstehend aufgeführten Modelle ist Schlaf als Freizeit berücksichtigt. Wie Biddle und Hamermesh (1990) hervorheben, wird in vielen Arbeitsangebotsmodellen davon ausgegangen, dass ein fester Zeitumfang auf Erwerbsarbeit und Freizeit im wachen Zustand verteilt wird. Schlaf ist damit implizit eine feste biologische Konstante, was jedoch weder durch die Theorie noch durch die Befunde gestützt wird. Biddle und Hamermesh zeigen theoretisch und empirisch auf, dass die Schlafenszeit, ebenso wie andere Formen der Zeitnutzung, auf marginale wirtschaftliche Anreize reagiert. Wenn dies der Fall ist, wird Schlaf im gewissen Umfang ebenfalls zu einer freizeitartigen Aktivität. Daher wurden in mehreren Erhebungen zur Zeitnutzung der jüngsten Zeit alle Schlafenszeiten als Freizeit eingestuft (Aguiar und Hurst, 2007; Engler und Staubli, 2008).

\section{Trendmäßige Entwicklung der Restgröße der Erwerbsarbeitszeit}

Die Analyse beginnt mit der Betrachtung der maximalen Freizeit als schlicht ausgedrückt die gesamte Zeit, die nicht mit Erwerbsarbeit verbracht wird. Zwar liegen einige Einschränkungen in Bezug auf diesen Ansatz unmittelbar auf der Hand - so bleiben etwa unentgeltliche Arbeit oder die Zeit für das Berufspendeln unberücksichtigt -, doch bietet er den Vorteil, dass Daten über die geleisteten Arbeitsstunden für eine Vielzahl von OECD-Ländern über lange Zeiträume hinweg auf vergleichbarer Basis vorhanden sind. Somit sind aussagefähige Vergleiche sowohl zwischen den Ländern als auch im Zeitverlauf möglich. Ausgehend von dieser ersten Definition von Freizeit als Restzeit, die nicht mit Arbeit verbracht wird, lässt sich nach und nach ein soliderer theoretischer Ansatz entwickeln, anhand dessen wiederum eine Analyse der Freizeitniveaus und -trends ermöglicht wird, die allerdings in Bezug auf die berücksichtigten OECD-Länder weniger umfassend ist.

Es ist möglich, die insgesamt geleisteten Jahresarbeitsstunden für Arbeitnehmer in Vollzeitäquivalenten für eine große Anzahl von OECD-Ländern zu schätzen und somit die entsprechende Restzeit zu berechnen (Tabelle 2.1). Eine offensichtliche Einschränkung in 
Tabelle 2.1. Analyse eines typischen Arbeitsjahrs für abhängig Beschäftigte, 2006

Zerlegung der tatsächlich geleisteten Jahresarbeitsstunden der Arbeitnehmer (in Volljahresäquivalenten) in ihre Komponenten

\begin{tabular}{|c|c|c|c|c|c|c|c|c|c|c|c|}
\hline & $\begin{array}{l}\text { Jahres- } \\
\text { arbeits- } \\
\text { stunden }{ }^{1}\end{array}$ & $\begin{array}{l}\text { Jährliche } \\
\text { Freizeit }\end{array}$ & $\begin{array}{l}\text { Durch- } \\
\text { schnittl. } \\
\text { Wochen- } \\
\text { arbeits- } \\
\text { stunden } \\
\text { in allen } \\
\text { Beschäf- } \\
\text { tigungen }\end{array}$ & $\begin{array}{l}\text { Übliche } \\
\text { Wochen- } \\
\text { arbeits- } \\
\text { stunden in } \\
\text { der Haupt- } \\
\text { beschäf- } \\
\text { tigung }\end{array}$ & $\begin{array}{c}\text { Zusätzl. } \\
\text { geleistete } \\
\text { Arbeits- } \\
\text { stunden in } \\
\text { der Haupt- } \\
\text { beschäf- } \\
\text { tigung = } \\
\text { Überstun- } \\
\text { den + } \\
\text { variable } \\
\text { Arbeits- } \\
\text { zeiten (z.B. } \\
\text { Gleitzeit) + } \\
\text { sonstige }\end{array}$ & $\begin{array}{c}\text { Arbeits- } \\
\text { stunden in } \\
\text { Neben- } \\
\text { beschäf- } \\
\text { tigungen }\end{array}$ & $\begin{array}{l}\text { Jahres- } \\
\text { arbeits- } \\
\text { wochen }\end{array}$ & $\begin{array}{c}\text { Feiertage } \\
\text { und } \\
\text { Urlaubs- } \\
\text { wochen }\end{array}$ & $\begin{array}{c}\text { Ganz- } \\
\text { wöchentl. } \\
\text { Abwesen- } \\
\text { heit aus } \\
\text { nicht } \\
\text { urlaubs- } \\
\text { bezoge- } \\
\text { nen } \\
\text { Gründen }\end{array}$ & $\begin{array}{c}\text { Abwesen- } \\
\text { heit eines } \\
\text { Teils der } \\
\text { Woche aus } \\
\text { nicht } \\
\text { urlaubs- } \\
\text { bezoge- } \\
\text { nen } \\
\text { Gründen }\end{array}$ & $\begin{array}{l}\text { Abwesen- } \\
\text { heit auf } \\
\text { Grund von } \\
\text { Krankheit } \\
\text { und Mutter- } \\
\text { schaft }^{2}\end{array}$ \\
\hline & $(a)=(c)^{\prime \prime}(g)$ & $\begin{array}{c}(b)= \\
(365 “ 24)-(a)\end{array}$ & $\begin{array}{c}(c)= \\
(d)+(e)+(f)\end{array}$ & (d) & (e) & $(\mathrm{f})$ & $\begin{array}{c}(\mathrm{g})=52- \\
{[(\mathrm{h})+(\mathrm{i})+} \\
(\mathrm{j})+(\mathrm{k})]\end{array}$ & (h) & (i) & (j) & (k) \\
\hline & & iden & Gelei & istete Woch & enarbeitsstur & nden & & leistete/nich & it geleistete $A$ & Arbeitswoch & hen \\
\hline Australien (2005) & 1733 & 7027 & 36.4 & .. & .. & .. & 47.6 & .. & .. & .. & .. \\
\hline Belgien & 1461 & 7299 & 36.0 & 35.4 & 0.3 & 0.4 & 40.5 & 7.1 & 2.2 & 0.4 & 1.8 \\
\hline Dänemark & 1367 & 7393 & 36.2 & 34.6 & 0.9 & 0.7 & 37.8 & 7.4 & 3.4 & 1.1 & 2.4 \\
\hline Deutschland & 1478 & 7282 & 36.1 & 34.3 & 1.4 & 0.3 & 41.0 & 7.5 & 1.7 & 0.6 & 1.1 \\
\hline Finnland & 1517 & 7243 & 38.6 & 36.9 & 1.2 & 0.4 & 39.4 & 7.1 & 2.4 & 1.6 & 1.5 \\
\hline Frankreich & 1459 & 7301 & 37.3 & 36.4 & 0.6 & 0.3 & 39.1 & 7.0 & 2.2 & 1.7 & 2.0 \\
\hline Griechenland & 1783 & 6977 & 40.0 & 39.6 & 0.1 & 0.3 & 44.5 & 6.7 & 0.3 & 0.2 & 0.3 \\
\hline Irland & 1543 & 7217 & 35.8 & 35.0 & 0.5 & 0.3 & 43.2 & 5.7 & 1.6 & 0.3 & 1.3 \\
\hline Island (2006) & 1748 & 7012 & 43.9 & 41.3 & 1.2 & 1.4 & 39.9 & 6.2 & 2.4 & 1.6 & 2.0 \\
\hline Italien & 1536 & 7224 & 37.3 & 36.8 & 0.3 & 0.2 & 41.2 & 7.9 & 1.4 & 0.3 & 1.2 \\
\hline Kanada (2005) & 1579 & 7181 & 36.3 & 35.6 & .. & 0.7 & 43.5 & 3.8 & 2.2 & 1.0 & 1.5 \\
\hline Luxemburg & 1541 & 7219 & 37.7 & 36.7 & 0.8 & 0.2 & 41.0 & 7.4 & 1.6 & 0.5 & 1.6 \\
\hline Niederlande & 1325 & 7435 & 31.6 & 29.5 & 1.6 & 0.5 & 41.9 & 5.3 & 2.2 & 0.9 & 1.6 \\
\hline Norwegen & 1290 & 7470 & 35.7 & 33.1 & 1.9 & 0.7 & 36.1 & 6.5 & 4.4 & 1.7 & 3.3 \\
\hline Österreich & 1590 & 7170 & 38.8 & 37.5 & 0.7 & 0.5 & 41.1 & 7.4 & 1.7 & 0.7 & 1.2 \\
\hline Polen & 1806 & 6954 & 41.5 & 40.0 & 0.4 & 1.1 & 43.5 & 6.2 & 1.3 & 0.1 & 1.0 \\
\hline Portugal & 1675 & 7085 & 40.0 & 39.0 & 0.2 & 0.7 & 41.9 & 7.3 & 1.5 & 0.2 & 1.1 \\
\hline Schweden & 1386 & 7374 & 37.5 & 35.6 & 1.3 & 0.6 & 36.9 & 6.8 & 3.3 & 1.8 & 3.2 \\
\hline Schweiz & 1618 & 7142 & 37.8 & 34.3 & 2.9 & 0.6 & 42.9 & 6.0 & 1.4 & 0.9 & 0.9 \\
\hline Slowak. Rep. & 1775 & 6985 & 40.8 & 40.3 & 0.3 & 0.2 & 43.5 & 6.9 & 0.7 & 0.1 & 0.7 \\
\hline Spanien & 1601 & 7159 & 39.1 & 38.2 & 0.6 & 0.3 & 41.0 & 6.8 & 1.9 & 0.5 & 1.8 \\
\hline Tschech. Rep. & 1754 & 7006 & 41.3 & 40.4 & 0.7 & 0.2 & 42.5 & 6.3 & 1.6 & 0.2 & 1.5 \\
\hline Ungarn & 1889 & 6872 & 41.3 & 40.6 & 0.3 & 0.4 & 42.6 & 6.2 & 1.5 & 0.2 & 1.4 \\
\hline Ver. Königreich & 1530 & 7230 & 37.5 & 36.6 & 0.6 & 0.3 & 40.8 & 6.5 & 2.1 & 1.3 & 1.2 \\
\hline Ver. Staaten ${ }^{3}(2005)$ & 1896 & 6864 & 41.3 & 38.5 & 2.7 & .. & 45.9 & 3.8 & 1.6 & .. & 0.7 \\
\hline OECD25 & 1595 & 7165 & 38.2 & 36.9 & 0.9 & 0.5 & 41.6 & 6.5 & 1.9 & 0.8 & 1.5 \\
\hline Variationskoeffizient & 0.11 & 0.02 & 0.07 & 0.08 & 0.81 & 0.62 & 0.06 & 0.16 & 0.44 & 0.75 & 0.47 \\
\hline
\end{tabular}

1. Vgl. Anhang 2.A1 des OECD Employment Outlook 2004 wegen einer knappen Erklärung der vom OECD-Sekretariat verwendeten Methode für die Schätzung der tatsächlich geleisteten Jahresarbeitsstunden je Erwerbstätigen in Belgien, Irland, Luxemburg, den Niederlanden und Portugal. Dieselbe Methode wird verwendet, um die geleisteten Jahresarbeitsstunden je Erwerbstätigen in allen in dieser Tabelle dargestellten europäischen Ländern zu schätzen.

2. Diese Wochen sind bereits in den Spalten $\mathrm{h}$ und $\mathrm{i}$ enthalten, werden jedoch ein zweites Mal berücksichtigt, um der angenommenen Untererfassung in Höhe von $50 \%$ (vgl. Anhang 2.A1) Rechnung zu tragen, mit Ausnahme Australiens.

3. Die Schätzungen beziehen sich auf die Vollzeitbeschäftigung insgesamt. Anstelle ganzwöchentlicher Abwesenheiten aus nicht urlaubsbezogenen Gründen wird die Gesamtzahl der Abwesenheitswochen ausgewiesen.

Quelle: Schätzungen des OECD-Sekretariats für die europäischen Länder auf der Grundlage der Ergebnisse der Europäischen Arbeitskräfteerhebungen und EIRO (2005). Schätzungen für Australien, Kanada, die Vereinigten Staaten auf der Grundlage von ECO/CPE/WP1(2007)11/ANN2. 
Bezug auf diesen Ansatz ist naturgemäß, dass er nichts - nicht einmal als Restgröße - über die Freizeit großer und unterschiedlicher Teile der Bevölkerung der einzelnen Länder aussagt, die nicht erwerbstätig sind. In Tabelle 2.1 sind zahlreiche interessante Befunde dargestellt. Erstens bestehen im Ländervergleich bei den geleisteten Jahresarbeitsstunden aller Beschäftigten erhebliche Unterschiede. Die Standardabweichung der Messgröße der Freizeit im Sinne von Restzeit zwischen den berücksichtigten Ländern beträgt 175 Stunden oder etwa vier Arbeitswochen à vierzig Arbeitsstunden pro Woche. Am wenigsten freie Restzeit gibt es in den Vereinigten Staaten, in Norwegen hingegen am meisten. Weitere Länder mit einem niedrigen Niveau an freier Restzeit sind Ungarn, Polen und die Slowakische Republik. Die meiste freie Restzeit ist in nordischen Ländern und im kontinentalen Westeuropa zu beobachten: in den Niederlanden, Dänemark, Schweden und Frankreich.

Wenn Freizeit lediglich als jene Zeit definiert wird, die nicht mit Erwerbsarbeit verbracht wird, dann schlägt sich natürlich jede Veränderung der geleisteten Jahresarbeitsstunden in Veränderungen der verfügbaren Freizeit nieder. In Tabelle 2.2 sind

Tabelle 2.2. Durchschnittliche jährliche Wachstumsrate der geleisteten Arbeitsstunden je Beschäftigten (in Vollzeitäquivalenten) für Fünfjahreszeiträume

\begin{tabular}{|c|c|c|c|c|c|c|c|}
\hline & $1970-1975$ & $1975-1980$ & $1980-1985$ & $1985-1990$ & 1990-1995 & $1995-2000$ & $2000-2005$ \\
\hline Australien & -0.2 & -0.2 & -0.1 & -0.1 & 0.0 & 0.0 & -0.7 \\
\hline Belgien & .. & .. & .. & -0.5 & -0.9 & -1.5 & 0.1 \\
\hline Dänemark & -2.4 & -0.3 & -0.5 & -1.1 & -0.2 & 0.7 & 0.3 \\
\hline Deutschland & .. & .. & .. & .. & .. & -0.8 & -0.5 \\
\hline Finnland & -0.9 & -0.5 & -0.4 & -0.5 & 0.1 & -0.3 & -0.4 \\
\hline Frankreich & -1.0 & -0.7 & -1.2 & -0.3 & -0.6 & -0.7 & -0.4 \\
\hline Griechenland & .. & .. & .. & -0.4 & 0.1 & 0.0 & -0.3 \\
\hline Irland & .. & .. & .. & 0.2 & -1.2 & -1.7 & -0.8 \\
\hline Island & -1.5 & -1.5 & -0.1 & -0.2 & -0.1 & 0.6 & -1.0 \\
\hline Italien & -1.3 & -0.6 & -0.6 & 0.1 & -0.5 & 0.0 & -0.5 \\
\hline Japan & -1.2 & 0.1 & -0.3 & -0.6 & -1.5 & -0.7 & -0.5 \\
\hline Kanada & -0.6 & -0.7 & -0.1 & 0.0 & -0.1 & -0.1 & -0.3 \\
\hline Korea & .. & .. & 0.1 & -1.5 & -0.2 & -1.1 & -1.4 \\
\hline Luxemburg & .. & .. & .. & 0.0 & -0.5 & -0.7 & -1.1 \\
\hline Mexiko & .. & .. & .. & .. & .. & 0.3 & 0.2 \\
\hline Neuseeland & .. & .. & .. & .. & 0.3 & -0.1 & -0.2 \\
\hline Niederlande & .. & .. & .. & .. & -1.6 & -0.3 & 0.0 \\
\hline Norwegen & -1.2 & -1.8 & -0.5 & -0.5 & -0.2 & -0.4 & -0.5 \\
\hline Österreich & .. & .. & .. & .. & .. & -0.2 & 0.3 \\
\hline Polen & .. & .. & .. & .. & .. & .. & 0.1 \\
\hline Portugal & .. & .. & .. & .. & -0.7 & -1.4 & -0.1 \\
\hline Schweden & -1.6 & -1.1 & 0.3 & 0.3 & 0.8 & 0.0 & -0.5 \\
\hline Schweiz & -1.0 & -0.8 & -0.8 & -0.4 & 0.0 & -0.2 & -0.3 \\
\hline Slowak. Rep. & .. & .. & .. & .. & .. & -0.7 & -0.8 \\
\hline Spanien & .. & .. & -1.5 & -0.3 & -0.1 & 0.0 & -0.5 \\
\hline Tschech. Rep. & .. & .. & .. & .. & .. & 0.3 & -0.9 \\
\hline Türkei & .. & .. & .. & .. & .. & .. & .. \\
\hline Ungarn & .. & .. & .. & -0.4 & 0.6 & 0.2 & -0.7 \\
\hline Ver. Königreich & -0.6 & -1.2 & -0.1 & 0.1 & -0.3 & -0.4 & -0.4 \\
\hline Ver. Staaten & -0.7 & -0.3 & 0.2 & -0.1 & 0.1 & -0.1 & -0.4 \\
\hline OECD30 & -1.1 & -0.7 & -0.4 & -0.3 & -0.3 & -0.3 & -0.4 \\
\hline
\end{tabular}

..: Nicht verfügbar.

Quelle: Schätzungen des OECD-Sekretariat auf der Grundlage des OECD-Beschäftigungsausblicks 2006. 
für den Zeitraum 1970-2005 die durchschnittlichen Wachstumsraten der Arbeitsstunden in den OECD-Ländern dargestellt. Bei den gewählten Fünfjahresdurchschnitten entfällt ein Großteil der möglichen kurzfristigen Konjunkturschwankungen. Die Daten sind offensichtlich unvollständig, bei vielen Ländern insbesondere für die Anfangszeit (19701985). Das Muster zeigt insgesamt jedoch einen Rückgang der geleisteten Arbeitsstunden, wobei sich das Tempo bei den meisten Ländern im Zeitverlauf verringert. Nur sehr wenige Länder haben Zeiträume verzeichnet, in denen die Zahl der geleisteten Arbeitsstunden pro Person gestiegen ist. Die Befunde scheinen sich nicht mit der Vorstellung einer allgemeinen OECD-weiten „Zeitknappheit“ zu decken, die sich aus den sich verändernden Bedingungen der Erwerbsbevölkerung resultiert, obgleich für bestimmte Gruppen sicherlich eine zunehmende Zeitknappheit gegeben sein könnte.

In Abbildung 2.1 werden anhand derselben Daten über einen Zeitraum von etwa dreißig Jahren die langfristigen Trends bei den geleisteten Jahresarbeitsstunden in sechs ausgewählten OECD-Ländern veranschaulicht. Kanada und die Vereinigten Staaten folgen sehr ähnlichen Mustern mit vergleichsweise stabilen Arbeitsstunden pro Person ab 1980. Die Muster des Vereinigten Königreichs sind denen der nordamerikanischen Staaten sehr ähnlich. Die Zahl der in Japan von den angestellten Beschäftigten geleisteten Arbeitsstunden ist stark zurückgegangen und konvergiert in Richtung des Niveaus der Vereinigten Staaten, Kanadas und des Vereinigten Königreichs. Aus den Daten für Frankreich und Norwegen geht für die 1980er und 1990er Jahre keine Stabilisierung hervor, für die Zeit nach der Jahrtausendwende ist dies jedoch in gewissem Maße der Fall.

\section{Abbildung 2.1. 1970-2006: langfristiger Rückgang der geleisteten Jahresarbeitsstunden}

Geleistete Jahresarbeitsstunden der gesamten Erwerbsbevölkerung in ausgewählten OECD-Ländern

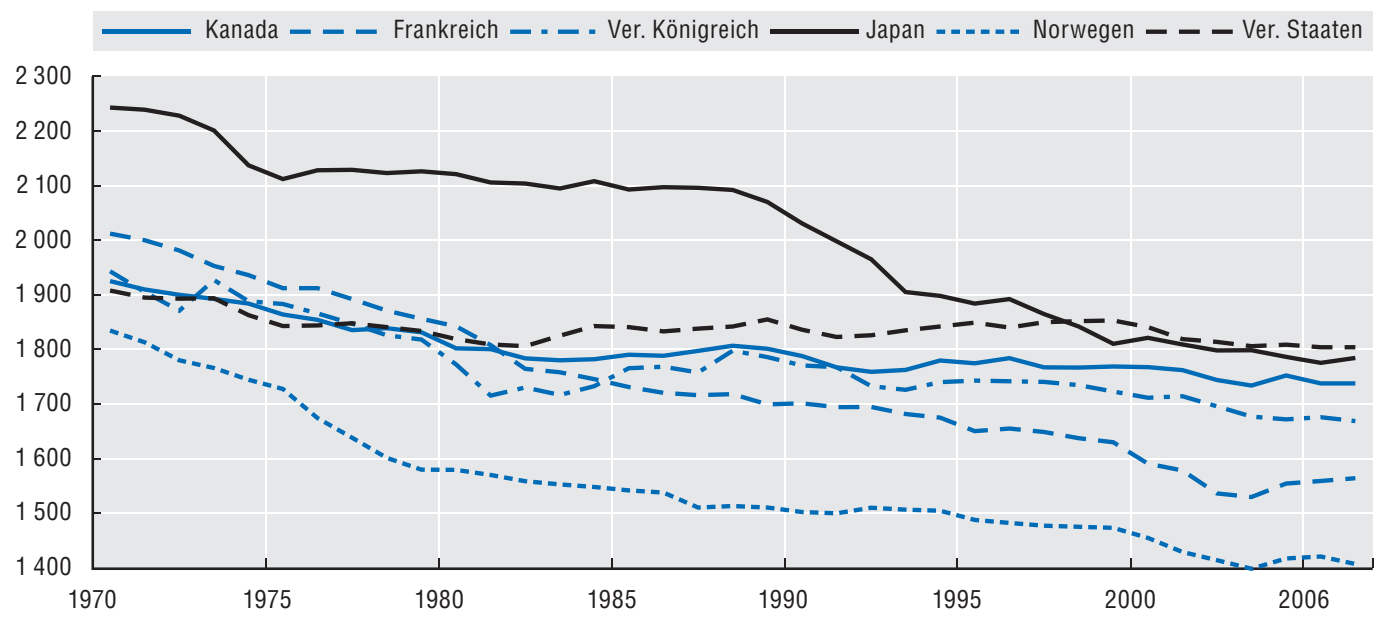

Quelle: Schätzungen des OECD-Sekretariats auf der Grundlage des OECD-Beschäftigungsausblicks 2006.

\section{Zeitallokation über den Lebenszyklus}

Eine zweite Kontextualisierung für die Analyse der Freizeitmuster anhand von Erhebungen zur Zeitnutzung ist die Betrachtung der Anzahl der Jahre, die Personen mit unterschiedlichen Merkmalen ihrer „Hauptaktivität“ in den verschiedenen Lebensphasen widmen. Diese Kontextualisierung beruht zwar auf Querschnittsdaten, kann jedoch unter der Annahme, dass die Erfahrungsmuster einer Person eines bestimmten Alters (z.B. 
15 Jahre) im Hinblick auf Arbeitsmarktergebnisse und Reproduktionsverhalten während eines bestimmten Zeitraums ihres künftigen Lebens (z.B. 15-64 Jahre) dem heutigen Verhalten der Bevölkerung dieser Altersgruppe (15-64 Jahre) entsprechen, die Muster der Zeitnutzung über den Lebenszyklus bis zu einem gewissen Grad erhellen. Die zu Grunde liegende Annahme ist dieselbe, auf der die Berechnung der Lebenserwartung oder der zusammengefassten Geburtenziffern basiert.

Die wichtigsten Ergebnisse sind in Abbildung 2.2 wiedergegeben, in der dargestellt ist, wie der Lebenszyklus einer Person nach in den unterschiedlichen Hauptaktivitätsphasen verbrachten Jahren aufgeschlüsselt werden kann. Diese Daten, die auf der Grundlage von Durchschnittswerten nach Männern und Frauen getrennt für die OECD-Länder dargestellt sind, für die genügend Daten verfügbar waren, verdeutlichen mehrere fest etablierte Muster. Das vielleicht bekannteste Muster in der Darstellung ist der stetige Rückgang der Anzahl der mit Erwerbsarbeit verbrachten Jahre bei den Männern und parallel dazu der entsprechende Anstieg bei den Frauen. Der Darstellung ist die Verlängerung der Ruhestandsphase infolge der steigenden Lebenserwartung zu entnehmen. Das frühere Renteneintrittsalter der Frauen und ihre längere Verweildauer im Ruhestand auf Grund des früheren Renteneintrittsalters und der längeren Lebenserwartung sind ebenfalls dargestellt. Der wahrscheinliche Anstieg der Verweildauer von Frauen in der Ausbildung ist hingegen nicht abgebildet. Zur isolierten Betrachtung des Aufholens der Frauen im Bildungsbereich sind weitere Arbeiten geplant.

\section{Abbildung 2.2. Anzahl der in einem typischen OECD-Land mit den verschiedenen Aktivitäten verbrachten Jahre, aufgeschlüsselt nach Männern und Frauen}
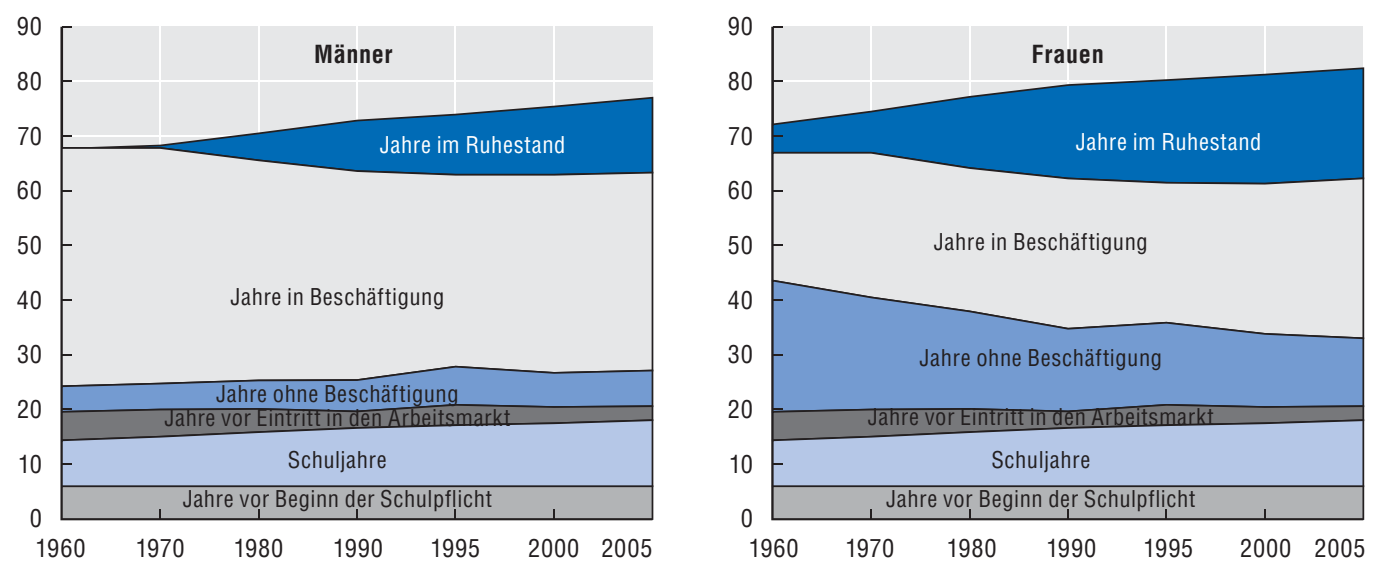

Quelle: Schätzungen des OECD-Sekretariats auf der Grundlage des OECD-Beschäftigungsausblicks 2006.

StatLink काIS http://dx.doi.org/10.1787/548526737374

\section{Freizeit an einem durchschnittlichen Tag}

Die vorstehende Analyse zeigt beträchtliche Unterschiede bei der durchschnittlich geleisteten Erwerbsarbeit in den verschiedenen OECD-Ländern pro Jahr. Des Weiteren ist die durchschnittliche Jahresarbeitszeit in den vergangenen dreißig Jahren in der Regel zurückgegangen. Aber ist der allgemeine Rückgang der geleisteten Jahresarbeitsstunden notwendigerweise mit einem symmetrischen Anstieg der verfügbaren Freizeit verbunden? Die Antwortet lautet nein. Die Schwächen des Restzeit-Ansatzes in Bezug auf die Erfassung der Bevölkerung wie auf die Annahme, dass die gesamte Restzeit in Form von Freizeit verbracht wird, sind offensichtlich. Diese Aufschlüsselung bietet letztlich nur einen 
teilweisen Einblick in die Elemente, aus denen sich ein typisches Jahr zusammensetzt. Für eine konkrete Schätzung der Zeit, die die Menschen speziell auf Freizeitaktivitäten verwenden, werden Daten benötigt, die nur durch Erhebungen zur Zeitnutzung verfügbar gemacht werden können.

Um ein besseres Verständnis für den Zeitanteil zu erlangen, den ein Mensch im Laufe eines typischen Jahres durchschnittlich pro Tag auf Freizeitaktivitäten verwendet, ist es wichtig, zunächst zu betrachten, wie Erwachsene diese 24 Stunden auf bestimmte Hauptaktivitäten aufteilen. Der hier gewählte Ansatz besteht darin, die Zeit entsprechend dem Tagesverlauf fünf Hauptkategorien zuzuordnen. Diese fünf Kategorien sind 1. Freizeit, eng definiert, 2. Erwerbsarbeit, 3. unentgeltliche Arbeit, 4. persönliche Pflege, 5. sonstige Zeitnutzung (Zeit, deren Nutzung entweder nicht erfasst oder nicht definiert ist). In dem Maße, wie die vollständige methodische Standardisierung und Vergleichbarkeit in Erhebungen zur Zeitnutzung erreicht werden kann, können im Ländervergleich bemerkenswerte Unterschiede in der Art und Weise beobachtet werden, wie die Menschen ihre tägliche Zeit im Durchschnitt einteilen. Dabei ist zu bedenken, dass die Ergebnisse von Erhebungen zur Zeitnutzung in Bezug auf die Messung von Tendenzen bei der Zeitallokation während Krankheits- und/oder Urlaubszeiten in unterschiedlichem Maße nicht als völlig exakt betrachtet werden können. Aktuelle Erhebungen zur Zeitnutzung mit genügend Daten für diese Studie sind ferner lediglich für 60\% der OECD-Länder verfügbar (die 18 in diesem Kapitel analysierten Länder), und ihre methodischen Ansätze sind relativ unterschiedlich. Leider waren keine ausreichenden Daten vorhanden, um die existierenden Erhebungen zur Zeitnutzung aus Island, den Niederlanden und Ungarn zu nutzen.

„Erwerbsarbeit“ umfasst Voll- und Teilzeitbeschäftigungen, Pausen am Arbeitsplatz, das Berufspendeln, die für die Arbeitsplatzsuche aufgewendete Zeit, die in einer Bildungseinrichtung verbrachte Zeit, die Fahrt zur Bildungseinrichtung und zurück sowie die zu Hause mit Erwerbsarbeit verbrachte Zeit. „Unentgeltliche Arbeit“ umfasst alle Arbeiten im Haushalt (Hausarbeit, Kochen, Putzen, Pflege von Kindern und anderen Familienmitgliedern und sonstigen Personen, ehrenamtliche Tätigkeiten, Einkaufen usw.). „Persönliche Pflege“ umfasst Schlafen, Essen und Trinken sowie sonstige Haushalts-, medizinische und persönliche Dienstleistungen (Hygiene, Körperpflege, Arzt- und Friseurbesuche usw.). „Freizeit“ umfasst Hobbys, Spiele, Fernsehen, Computernutzung, Hobbygärtnern, Sport, soziale Begegnungen mit Freunden und Familie, Besuch von Veranstaltungen usw. „Sonstige Zeitnutzung“ umfasst alle Aktivitäten, die nicht in einer anderen Kategorie aufgeführt sind.

Aus Abbildung 2.3 geht hervor, dass die Menschen in allen 18 OECD-Ländern am meisten Zeit für Aktivitäten der persönlichen Pflege aufwenden. Die Unterschiede bei den Zeitanteilen, die in diesen Ländern auf die persönliche Pflege verwendet werden, sind mit 6 Prozentpunkten vergleichsweise gering, wobei die Anteile von $43 \%$ der gesamten Zeit in Kanada, Schweden, Mexiko und Norwegen bis zu 49\% in Frankreich reichen.

Aus welchen Aktivitäten setzt sich die persönliche Pflege hauptsächlich zusammen? Die Hauptkomponente der persönlichen Pflege ist in allen Ländern effektiv der Schlaf. Im Durchschnitt der OECD-Länder schlafen die Menschen 8 Stunden und 22 Minuten pro Tag. Auf Schlaf entfallen somit etwa 77\% der durchschnittlich im OECD-Raum für die persönliche Pflege aufgewendeten Zeit. Die zweite große Komponente ist Essen, worauf 


\section{Abbildung 2.3. Zeitanteile für Freizeit und andere Aktivitäten im Laufe eines Durchschnittstags}

Aufschlüsselung der über 24 Stunden mit den Hauptaktivitäten verbrachten Zeit für alle Befragten ab 15 Jahre in 18 OECD-Ländern

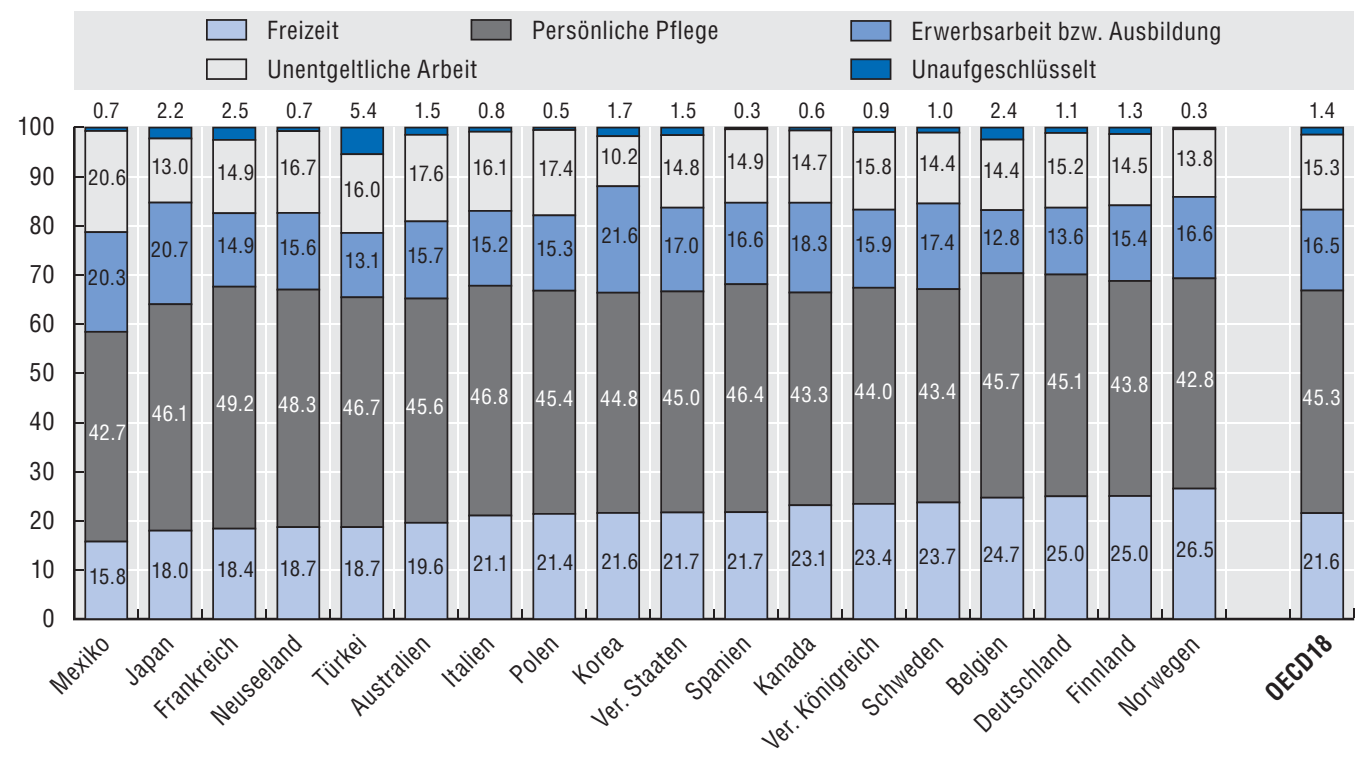

Quelle: Schätzungen des OECD-Sekretariats auf der Grundlage nationaler und multinationaler Erhebungen zur Zeitnutzung (2006, sofern verfügbar).

StatLink त्ञाज http://dx.doi.org/10.1787/548528164155

weitere 14\% der für die persönliche Pflege aufgewendeten Zeit bzw. - im Durchschnitt der OECD-Länder - 1 Stunde und 37 Minuten pro Tag entfallen (die Türkei ist hierin und in den nachfolgenden Berechnungen nicht enthalten, da die Essenszeit nicht von anderen Zeiten für die persönliche Pflege getrennt werden kann). Schlafen und Essen nehmen demnach im Durchschnitt über $90 \%$ der Zeit für die persönliche Pflege ein. Die restliche Zeit für die persönliche Pflege deckt „persönliche, medizinische und Haushaltsdienstleistungen“ ab. In dieser letztgenannten Kategorie sind unterschiedliche Aktivitäten wie persönliche Hygiene, Arzt- und Friseurbesuche, Autoreparaturen usw. erfasst. Da ein gewisser Anteil der Zeit für Schlafen, Essen und Trinken sowie für die persönliche Hygiene alternativ als Freizeit eingestuft werden könnte (z.B. für Ausschlafen, ein langes Mittagessen mit Freunden bzw. Familie oder einen Friseurbesuch), besteht ein erheblicher Grad von Willkür bei der Trennung zwischen persönlicher Pflege und Freizeit.

Nach der persönlichen Pflege ist Freizeit im Allgemeinen die nächstgrößte Kategorie, auf die im Durchschnitt der OECD18 ein Anteil von 22\% der Zeit entfällt. Der Freizeitanteil ist mit 27\% der Zeit in Norwegen am höchsten, in Mexiko mit 16\% der Zeit am niedrigsten. Auch in Belgien, Deutschland und Finnland sind die Freizeitanteile hoch. Am anderen Ende des Spektrums ist die Freizeit in Japan, Frankreich und Neuseeland ebenfalls vergleichsweise gering.

Japan und Mexiko sind die einzigen beiden Länder, in denen größere Zeitanteile auf Erwerbsarbeit entfallen als auf Freizeit, während Erwerbsarbeit und Freizeit sich in Korea die Waage halten. Die anderen 15 Länder weisen allesamt mehr Freizeit als Erwerbsarbeitszeit aus. Im Durchschnitt der OECD18 steht die Erwerbsarbeit in der Rangfolge unter der Freizeit, die Differenz ist jedoch gering. In vielen Ländern, z.B. in Mexiko, Neuseeland, Australien, Italien, Polen, Belgien und Deutschland nimmt 
unentgeltliche Arbeit effektiv mehr Zeit ein als Erwerbsarbeit. Mexiko ist das einzige Land, in dem unentgeltliche Arbeit auch mehr Zeit einnimmt als Freizeit.

Unter den vier größten Zeitkategorien (Freizeit, persönliche Pflege, Erwerbsarbeit und unentgeltliche Arbeit) bestehen die deutlichsten Unterschiede im Ländervergleich bei dem Anteil, der auf die Freizeit entfällt: 11 Prozentpunkte beträgt hier die Differenz zwischen Mexiko und Norwegen. Ebenso hoch ist der Unterschied bei der unentgeltlichen Arbeit mit einer Differenz von 11 Prozentpunkten zwischen Korea (niedrig) und Mexiko (hoch). Bei der Erwerbsarbeit ist die Differenz geringer und beträgt 9 Prozentpunkte zwischen Belgien (niedrig) und Korea (hoch).

In Anbetracht des vorstehend bereits erörterten willkürlichen Charakters der Grenze zwischen persönlicher Pflege und Freizeit besteht eine alternative Möglichkeit zur Messung der mit Freizeitaktivitäten verbrachten Zeit darin, die persönliche Pflege auf den niedrigsten Wert auf Länderebene festzusetzen (im Durchschnitt 42,7\% des Tages in Mexiko). Es ließe sich argumentieren, dass dieser niedrigste Länderwert für die persönliche Pflege dem notwendigen Minimum entspricht. Was als „breit abgegrenzte“ Definition der Freizeit beschrieben wird, kann dann als bereits gemessene und in Abbildung 2.3 oben dargestellte „eng abgegrenzte“ Freizeit plus der zusätzlichen „überschüssigen“ Zeit für die persönliche Pflege, die über dem Wert des Landes mit dem niedrigsten Anteil liegt, berechnet werden. Die Ergebnisse dieser Berechnung der „breit abgegrenzten Freizeit“ sind in Abbildung 2.4 dargestellt. Die durchschnittliche Freizeit erhöht sich für die OECD18 von 21,6\% der Zeit („eng abgegrenzte“ Freizeit) auf 24\% der Zeit („breit abgegrenzte“ Freizeit). Die Differenz beträgt nach wie vor 11 Prozentpunkte zwischen dem Tiefstwert von im Durchschnitt $16 \%$ des Tages in Mexiko, die mit Freizeit verbracht werden, und dem Höchstwert von im Durchschnitt 27\% des Tages in Belgien, doch bei anderen Ländern innerhalb dieser Bandbreite ist eine größere Homogenität vorhanden.

\section{Abbildung 2.4. Eine breiter abgegrenzte Definition der Freizeit erhöht die Freizeitanteile und verändert die Länderrangfolge}

Prozentsatz der Freizeit an einem Durchschnittstag

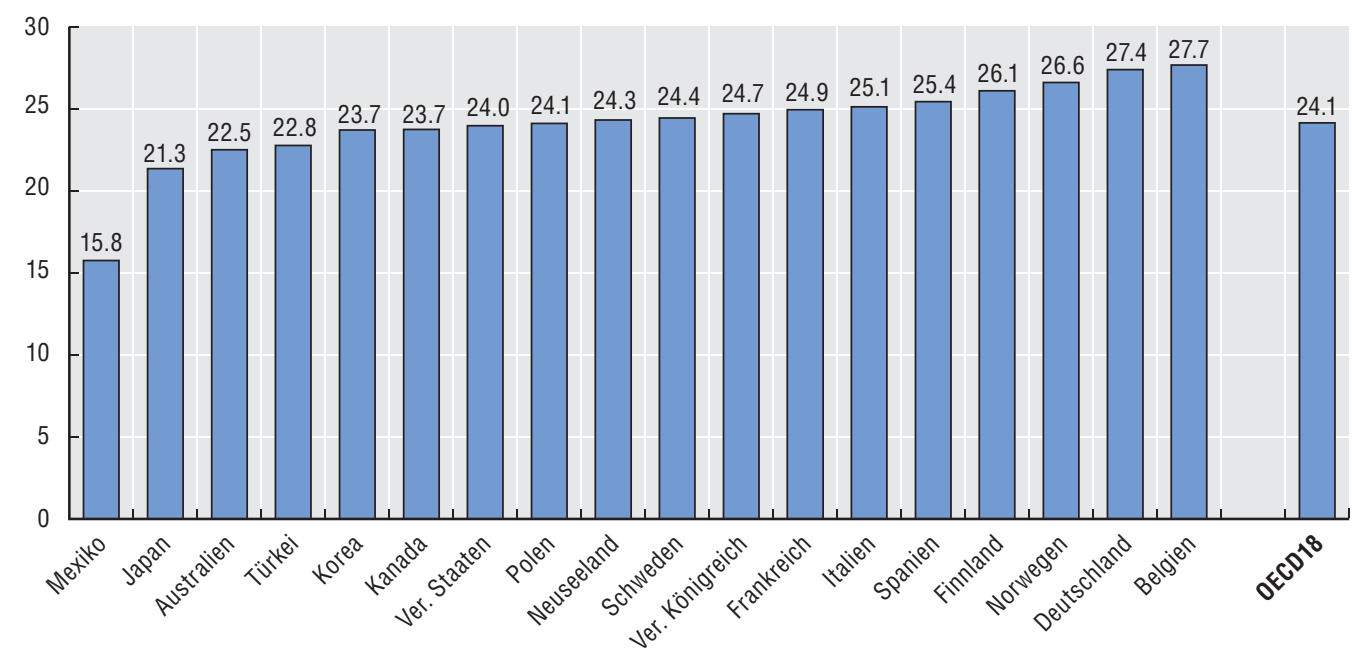

Anmerkung: „Breit abgegrenzte Freizeit“ bezieht sich auf den auf das niedrigste Niveau auf Länderebene normierten Tagesumfang der persönlichen Pflege. Die darüber hinausgehend auf die persönliche Pflege verwendete Zeit wird auf den ursprünglichen Wert für die Freizeit angerechnet.

Quelle: Schätzungen des OECD-Sekretariats auf der Grundlage nationaler und multinationaler Erhebungen zur Zeitnutzung (2006, sofern verfügbar). 
Einige Länder gewinnen bei Verwendung der breit abgegrenzten Definition mehr Freizeit als andere. In der Folge ergeben sich bei den Länderrangfolgen ebenfalls deutliche Veränderungen. Die Länder, die in der Rangfolge am stärksten aufrücken, sind Frankreich (um neun Plätze), Italien (um sechs Plätze) und Neuseeland (um fünf Plätze). Diese drei Länder bewegen sich von unterdurchschnittlichen Werten zu Werten um oder über dem OECD-Durchschnitt im Bereich der Freizeit. Zu den Ländern, die in Bezug auf die breit abgegrenzte Freizeit in der Rangfolge am stärksten abfallen, zählen Kanada (um sechs Plätze) sowie Schweden und Korea (jeweils um vier Plätze). Im Fall Frankreichs ist der unmittelbare Grund für die große Veränderung der sehr hohe Zeitanteil für die persönliche Pflege - mit 49\% der Zeit der höchste im OECD-Raum -, von dem ein Teil nun der Freizeit zugeordnet wird. Im Hinblick auf die Zusammensetzung dieses hohen Zeitanteils für die persönliche Pflege ist das hohe Maß an nächtlichem Schlaf der Franzosen von Interesse (der, wie oben erwähnt, der persönlichen Pflege zugerechnet wird). Die Daten über das Schlafgebaren in den OECD-Ländern sind in Abbildung 2.5 dargestellt. Im Durchschnitt schlafen die Franzosen pro Tag mehr als eine Stunde länger als die Koreaner, die im OECDRaum mit dem wenigsten Schlaf auskommen.

\section{Abbildung 2.5. Die Franzosen schlafen länger}

Schlafdauer an einem Durchschnittstag in Minuten

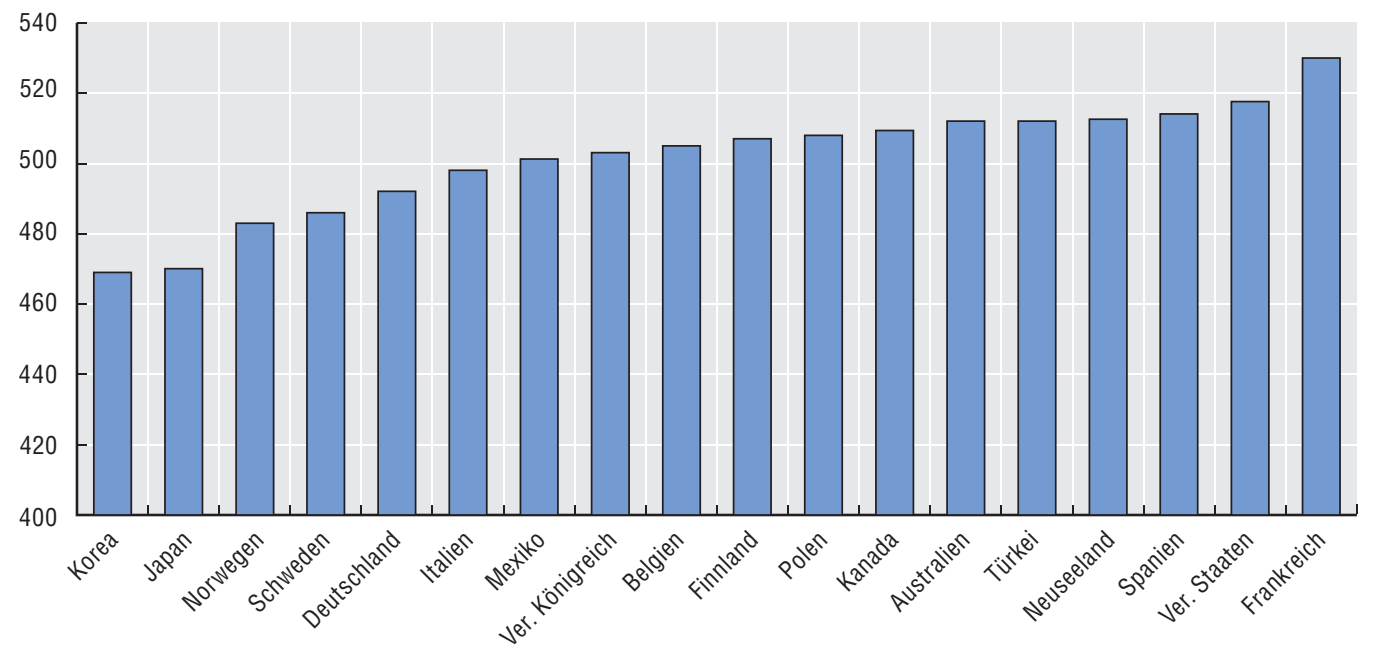

Quelle: Schätzungen des OECD-Sekretariats auf der Grundlage nationaler und multinationaler Erhebungen zur Zeitnutzung (2006, sofern verfügbar).

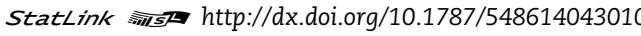

Eine weitere wichtige Aktivität im Bereich der persönlichen Pflege, die - wie bereits erwähnt - in vielen Kulturen eine freizeitartige Qualität hat, ist das Essen. Abbildung 2.6 verdeutlicht, dass die Differenz bei der mit Essen zugebrachten Zeit zwischen dem Land mit dem höchsten und jenem mit dem niedrigsten Wert nahezu eineinhalb Stunden beträgt. Die Länder, die in der Rangfolge der breit abgegrenzten Freizeit den deutlichsten Sprung nach oben gemacht haben, d.h. Frankreich und Neuseeland, weisen beide auch lange Essenszeiten auf. Die Franzosen verwenden täglich nahezu doppelt so viel Zeit auf das Essen wie die Menschen in Mexiko, Kanada und den Vereinigten Staaten. 


\section{Abbildung 2.6. Die Franzosen essen und trinken am längsten}

Essensdauer an einem Durchschnittstag in Minuten

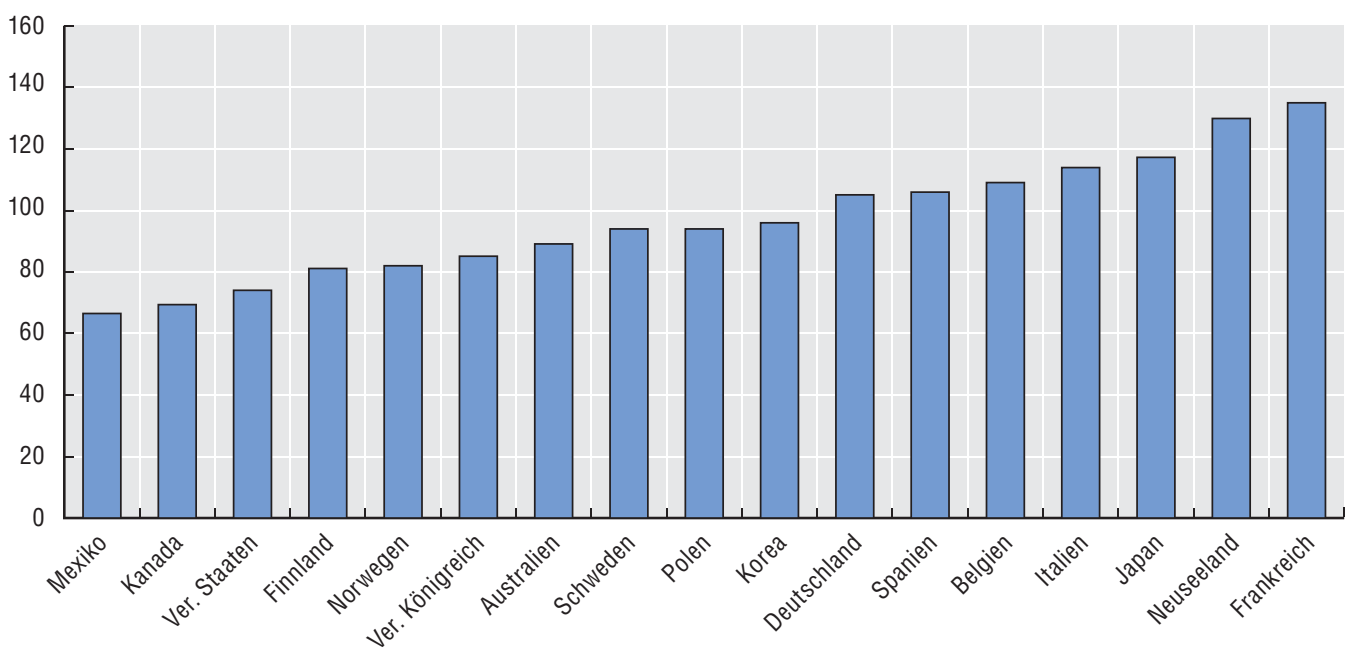

Anmerkung: Bei den verfügbaren Daten aus Erhebungen zur Zeitnutzung für die Türkei erfolgt keine Trennung zwischen persönlicher, medizinischer und Haushaltspflege sowie Essen und Trinken. Die Zahlen für die Türkei sind daher hier unberücksichtigt. Eine Ad-hoc-Abtrennung der Zeiten für Essen und Trinken auf der Grundlage der Durchschnittsanteile der OECD-Länder ergäbe für die Türkei einen Wert, der ungefähr auf dem italienischen Niveau läge.

Quelle: Schätzungen des OECD-Sekretariats auf der Grundlage nationaler und multinationaler Erhebungen zur Zeitnutzung (2006, sofern verfügbar).

StatLink ताISL http://dx.doi.org/10.1787/548621131285

Die letzte und kleinste Kategorie der persönlichen Pflege stellen die „persönlichen, medizinischen und Haushaltsdienstleistungen“ dar. Die für diese Aktivitäten aufgewendete Zeit schwankt wiederum erheblich und reicht von 43 Minuten pro Tag in Finnland bis zu 77 Minuten pro Tag in Korea (Abbildung 2.7).

\section{Abbildung 2.7. In Japan und Korea wird mehr Zeit auf persönliche, medizinische und Haushaltsdienstleistungen verwendet}

Auf persönliche, medizinische und Haushaltsdienstleistungen verwendete Zeit an einem Durchschnittstag in Minuten

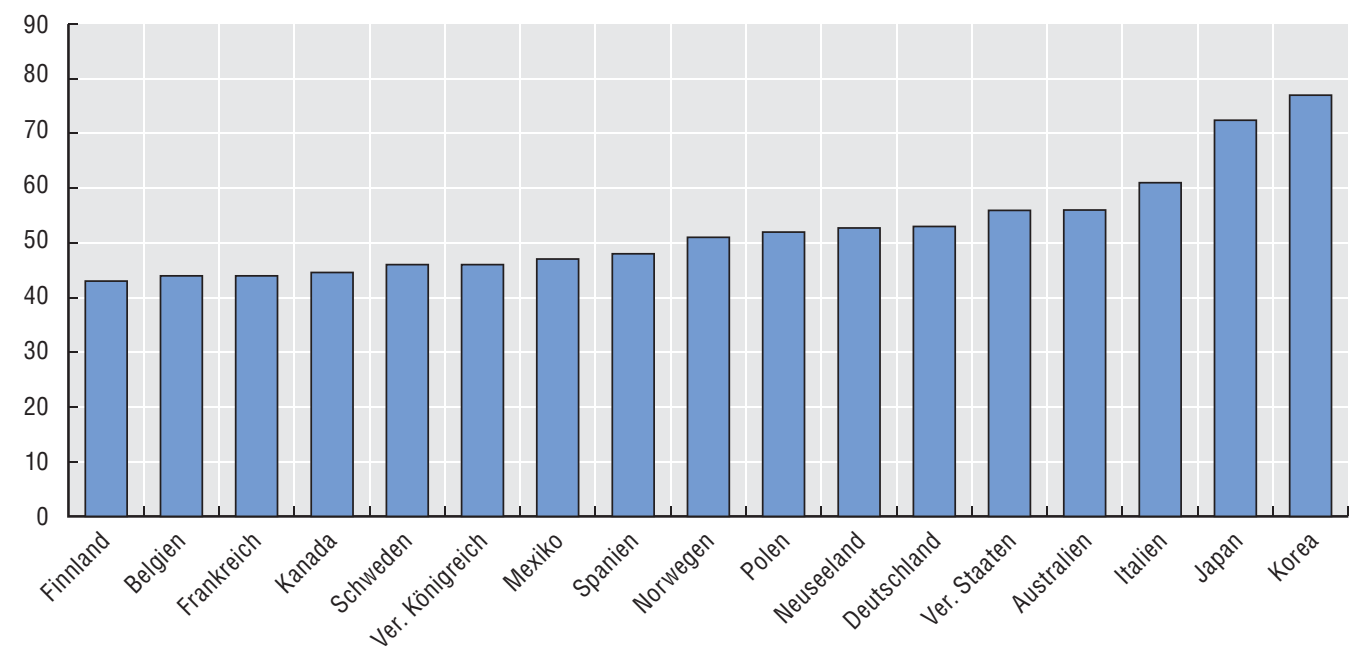

Quelle: Schätzungen des OECD-Sekretariats auf der Grundlage nationaler und multinationaler Erhebungen zur Zeitnutzung (2006, sofern verfügbar). 


\section{Entwicklungen im Zeitverlauf im Bereich Freizeit auf der Grundlage der Erhebungen zur Zeitnutzung}

Eine weitere interessante Frage betrifft die Muster der Veränderung der Freizeit im Zeitverlauf für alle Erwachsenen (unter Verwendung einer eng abgegrenzten Messgröße). Diese Frage kann für die wenigen Länder behandelt werden, die über einen ausreichend langen Zeitraum Erhebungen zur Zeitnutzung durchgeführt haben. Bei diesen Ländern handelt es sich um Kanada, die Niederlande, Norwegen, das Vereinigte Königreich und die Vereinigten Staaten ${ }^{2}$. Für jedes Land sind Langzeitdaten für Zeiträume unterschiedlicher Höchstdauer und Frequenz vorhanden. In allen Fällen ist die Frequenz niedrig, so dass Schlussfolgerungen über langfristige Entwicklungen im Zeitverlauf mit Vorsicht zu ziehen sind. Abbildung 2.8 zeigt, dass es in den vorstehend genannten Ländern in den vergangenen vierzig Jahren unterschiedliche Entwicklungen bezüglich der auf die Freizeit verwendeten Zeitanteile gegeben hat. Der auf Freizeit entfallende Zeitanteil scheint in den Niederlanden von Mitte der 1970er Jahre bis Anfang des 21. Jahrhunderts zurückgegangen zu sein, und im Vereinigten Königreich scheint sich im Hinblick auf die Freizeit eine ähnliche, wenn auch weniger stark ausgeprägte rückläufige Entwicklung vollzogen zu haben. In Norwegen ist die Freizeit über den gesamten Zeitraum, für den Daten vorhanden sind, im Wesentlichen unverändert geblieben. In Kanada und den Vereinigten Staaten schließlich, für die Daten über die längsten Zeiträume verfügbar sind, nimmt der Freizeitanteil zu, wenngleich das Ausgangsniveau für diese Entwicklung wesentlich niedriger ist als in den europäischen OECD-Ländern. Leider sind in Bezug auf Quantität und Frequenz keine ausreichenden Daten vorhanden, um Freizeit im Zusammenhang mit dem Konjunkturzyklus zu betrachten, was es erlaubt hätte, freiwillige und unfreiwillige Veränderungen im Bereich der Freizeit zu erhellen.

\section{Abbildung 2.8. Entwicklungen im Zeitverlauf im Bereich Freizeit auf der Grundlage von Zeitnutzungserhebungen}

Langfristige Entwicklungen im Bereich der Freizeit an einem Durchschnittstag in fünf ausgewählten OECD-Ländern, in Prozent

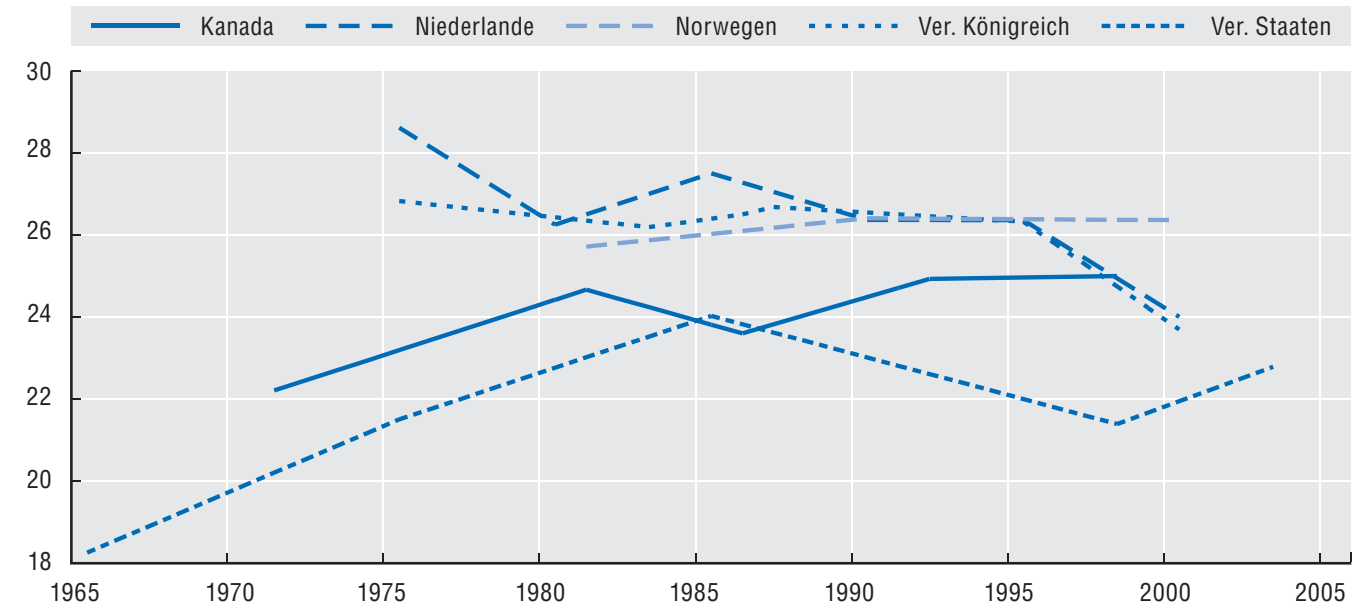

Quelle: Schätzungen des OECD-Sekretariats auf der Grundlage nationaler und multinationaler Erhebungen zur Zeitnutzung (2006, sofern verfügbar). 


\section{Muster der Freizeitverteilung}

\section{Demografische Merkmale und Freizeit}

Auf welche Weise unterscheidet sich die mit Freizeitaktivitäten verbrachte Freizeit in den verschiedenen gesellschaftlichen Gruppen? In diesem Abschnitt werden unterschiedliche Freizeitmuster für verschiedene gesellschaftliche Gruppen nach Geschlecht und Alter getrennt betrachtet.

\section{Geschlecht}

Was für Unterschiede bestehen in Bezug auf den Umfang der Freizeit zwischen Männern und Frauen im OECD-Raum? Geschlechtsspezifische Unterschiede im Hinblick auf Erwerbsarbeit, unentgeltliche Arbeit und Arbeit insgesamt sind bereits in hohem Maße Gegenstand vergleichender Arbeiten gewesen. Geschlechtsspezifischen Unterschieden im Freizeitbereich ist jedoch weitaus weniger Aufmerksamkeit zuteil geworden. Burda et al. (2007) stellen nach der Auswertung von Daten zur Zeitnutzung für Belgien, Dänemark, Deutschland, Finnland, Frankreich, Italien, die Niederlande, Norwegen, Schweden, das Vereinigte Königreich und die Vereinigten Staaten fest, dass „die geschlechtsspezifischen Unterschiede hinsichtlich des Umfangs der in Anspruch genommenen Freizeit in den meisten reichen Volkswirtschaften verschwindend gering sind“ (S. 23). Es ließe sich darüber streiten, ob die in der Studie von Burda et al. ausgewiesenen Unterschiede zwischen Männern und Frauen tatsächlich so gering sind. Die Autoren erfassen in der Größenordnung von Minuten geschlechtsspezifische Unterschiede für einen Durchschnittstag, der repräsentativ für ein ganzes Jahr sein soll. Auf Tagesbasis erscheint die Differenz von Minuten in der Regel gering. Aufs Jahr gesehen, sieht die Sache jedoch anders aus. Die geringste Differenz beläuft sich demnach auf 55 Stunden im Jahr zu Gunsten der Freizeit der Männer in Norwegen. Es ist unwahrscheinlich, dass eine zusätzliche arbeitsfreie Zeit von mehr als einer Woche im Jahr für die meisten vollzeitbeschäftigten Arbeitnehmer von „verschwindend geringer“ Bedeutung wäre. Die Hochrechnung der bei Burda et al. (Tabelle 1.1 und 1.2) auf Tagesbasis angegebenen geschlechtsspezifischen Freizeitdifferenzen - alle zu Gunsten der Männer - auf das Jahr liefert Zahlen von 116 Stunden pro Jahr in den Niederlanden, 128 Stunden in den Vereinigten Staaten, 134 Stunden in Schweden, 170 Stunden im Vereinigten Königreich, 176 Stunden in Deutschland, 195 Stunden in Dänemark, 213 Stunden in Belgien, 225 Stunden in Finnland, 280 Stunden in Frankreich und 444 Stunden in Italien.

Auf der Grundlage der Erhebungen zur Zeitnutzung für die in Abbildung 2.9 wiedergegebenen 18 OECD-Länder geben Männer durchweg an, mehr Zeit mit Aktivitäten zu verbringen, die im engeren Sinne als Freizeit definiert sind, als dies bei Frauen der Fall ist, was den Ergebnissen von Burda et al. entspricht. Die geschlechtsspezifischen Unterschiede sind in Norwegen diesbezüglich statistisch gesehen trivial (einige Minuten pro Tag). Im Gegensatz hierzu verfügen die Frauen in Italien über nahezu 80 Minuten weniger Freizeit pro Tag als die Männer. Burda et al. (2007, S. 4-5) haben bereits den großen Umfang an unentgeltlicher Arbeit bemerkt, die die italienischen Frauen verrichten, ebenso wie die viele Zeit, die die italienischen Männer vor dem Fernseher verbringen. Dementsprechend wird offenbar ein Großteil der zusätzlichen Arbeit der italienischen Frauen auf das Putzen zu Hause verwandt.

Allerdings schwanken diese geschlechtsspezifischen Unterschiede bei der Freizeit, je nachdem auf welche Art und Weise Zeit als „Freizeit“ oder „persönliche Pflege“ eingestuft 


\section{Abbildung 2.9. Männer verfügen über mehr Freizeit als Frauen}

Geschlechtsspezifische Unterschiede im Bereich Freizeit, Minuten pro Tag, positive Zahlen stellen einen Vorteil zu Gunsten der Männer dar

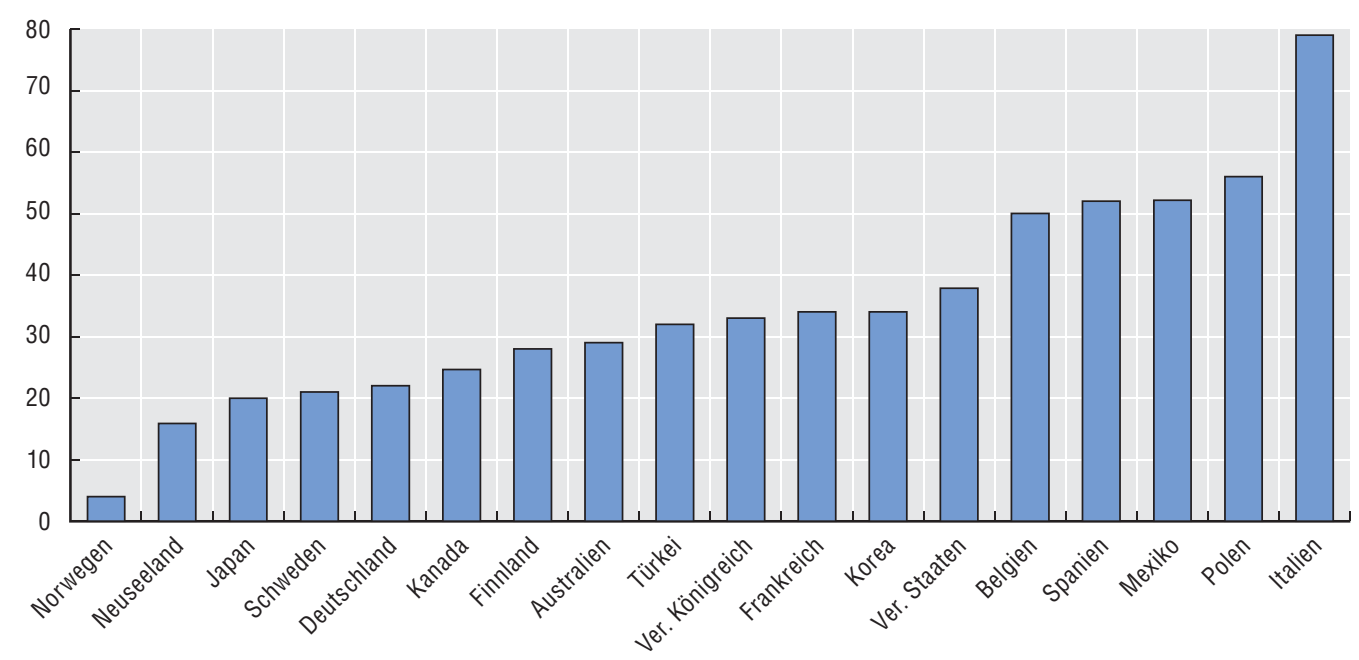

Anmerkung: Die eng abgegrenzte Definition der Freizeit wurde verwendet.

Quelle: Schätzungen des OECD-Sekretariats auf der Grundlage nationaler und multinationaler Erhebungen zur Zeitnutzung (2006, sofern verfügbar).

StatLink काजिए http://dx.doi.org/10.1787/548724153767

\section{Abbildung 2.10. Männer verwenden im Allgemeinen weniger Zeit auf die persönliche Pflege als Frauen}

Geschlechtsspezifische Unterschiede bei den Zeitanteilen für die persönliche Pflege, Minuten pro Tag, positive Zahlen stellen einen Vorteil zu Gunsten der Männer dar

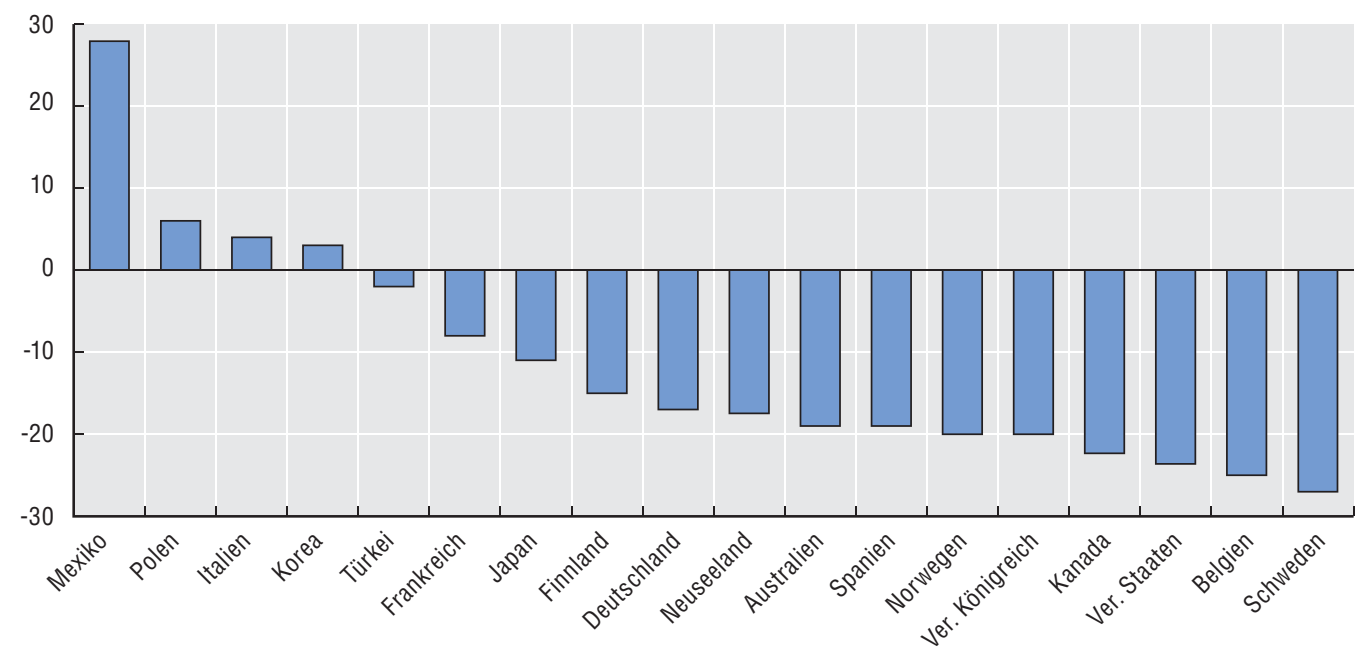

Quelle: Schätzungen des OECD-Sekretariats auf der Grundlage nationaler und multinationaler Erhebungen zur Zeitnutzung (2006, sofern verfügbar).

StatLink तiाs $h$ ttp://dx.doi.org/10.1787/548733815678

wird. Welche geschlechtsspezifischen Unterschiede bestehen also im Hinblick auf die persönliche Pflege? Abbildung 2.10 zeigt, dass Frauen in den meisten OECD-Ländern mehr Minuten pro Tag auf die persönliche Pflege verwenden als Männer, in einigen Fällen - etwa in Schweden, Belgien und den Vereinigten Staaten - sogar wesentlich mehr. Die Länder, in denen die Männer mehr Zeit auf die persönliche Pflege verwenden als Frauen, sind Italien, 
Polen, Korea und Mexiko. Mit nahezu einer halben Stunde pro Tag ist die Differenz im Fall Mexikos besonders groß. Der Großteil der Differenz in Mexiko ist darauf zurückzuführen, dass die Männer pro Tag 25 Minuten länger schlafen als die Frauen (die Schlafdauer der Mexikaner - Männer und Frauen zusammen - liegt leicht unter dem OECD-Tagesdurchschnitt).

Um die Unterschiede im Rahmen einer breiter abgegrenzten Definition der Freizeit zu untersuchen, wird die täglich für die persönliche Pflege aufgewendete Zeit wiederum auf den niedrigsten Landeswert normiert (602 Minuten bei mexikanischen Frauen). Die darüber hinaus von Männern wie Frauen auf die persönliche Pflege aufgewendete Zeit wird dann wiederum dem Freizeitbereich zugerechnet. Diese Neuverteilung führt zu einer breiteren und möglicherweise besseren Messung der Freizeitunterschiede zwischen Männern und Frauen (Abbildung 2.11).

\section{Abbildung 2.11. Männer verfügen im Allgemeinen über mehr breit abgegrenzte Freizeit als Frauen}

Geschlechtsspezifische Unterschiede bei der breit abgegrenzten Freizeit, Minuten pro Tag, positive Zahlen stellen einen Vorteil zu Gunsten der Männer dar

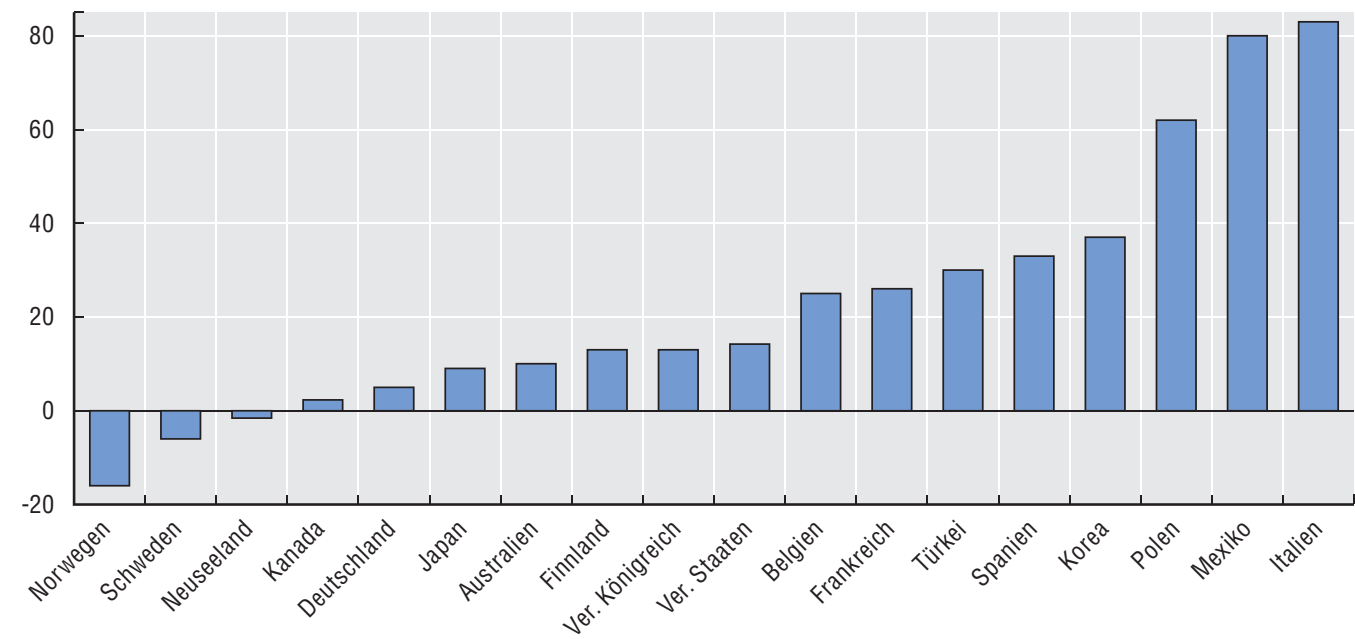

Anmerkung: „Breit abgegrenzte Freizeit“ bezieht sich auf die auf das niedrigste Niveau auf Länderebene normierten täglichen geschlechtsspezifischen Anteile der persönlichen Pflege, und die darüber hinaus auf die persönliche Pflege verwendete Zeit wird auf den ursprünglichen Wert für die Freizeit angerechnet.

Quelle: Schätzungen des OECD-Sekretariats auf der Grundlage nationaler und multinationaler Erhebungen zur Zeitnutzung (2006, sofern verfügbar).

StatLink ants http://dx.doi.org/10.1787/548741477728

Trotz dieser Bereinigung um die freizeitartige persönliche Pflege verwenden die Männer in den meisten Ländern nach wie vor mehr Zeit auf Freizeitaktivitäten im weiteren Sinne als Frauen. In Italien und Mexiko ist der Abstand besonders groß. Allerdings gibt es nun auch drei Länder, in denen die Frauen über mehr Freizeit im Sinne der breiter abgegrenzten Definition verfügen: Neuseeland, Norwegen und Schweden. Von praktischer Bedeutung ist der Vorsprung nur für die Frauen in Norwegen, die im Durchschnitt täglich 16 Minuten mehr Freizeit haben als Männer. Die Freizeitdifferenz zu Gunsten der Männer ist in mehreren Ländern jedoch nach wie vor sehr erheblich, insbesondere in Italien, Mexiko, Polen und Korea, und in anderen Ländern ist sie zumindest immer noch groß. Es ist bemerkenswert, dass Italien, Mexiko, Polen und Korea Länder sind, in denen die bereits bei der eng abgegrenzten Freizeit bestehende Differenz nach Geschlecht sich bei Berücksichtigung der geschlechtsspezifischen Differenz bei der persönlichen Pflege noch 
vergrößert. Somit verfügen Männer - unabhängig davon, ob Freizeit breit oder eng abgegrenzt wird - in den meisten Ländern in der Regel über mehr Freizeit als Frauen ${ }^{3}$.

Eine verbleibende Einschränkung bei der Betrachtung geschlechtsspezifischer Differenzen im Bereich der Freizeit ergibt sich aus dem möglicherweise geschlechtsspezifischen Charakter des Einkaufens als Freizeitaktivität. Bei der vorstehenden Analyse wird das Einkaufen vollständig der unentgeltlichen Arbeit zugerechnet. Unter sonst gleichen Bedingungen wird auf diese Weise die Freizeit verringert. Aus den Erhebungen zur Zeitnutzung ist ebenfalls bekannt, dass Frauen länger einkaufen als Männer. In den Vereinigten Staaten etwa verbringen Männer 43 Minuten pro Tag mit dem Einkaufen, Frauen hingegen 59 Minuten pro Tag. Die entsprechenden Zahlen lauten für Deutschland 49 Minuten bei den Männern und 66 Minuten bei den Frauen, für Italien 33 Minuten bei den Männern und 53 Minuten bei den Frauen und für die Niederlande 36 Minuten bei den

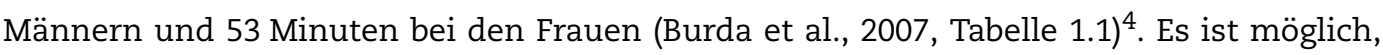
dass ein Teil dieser Einkaufszeit eine gewisse Freizeitkomponente aufweist und dieses freizeitartige Einkaufen bei Frauen in der Regel mehr Raum einnimmt.

\section{Freizeitmuster nach Alter}

Um ein reales Bild der Freizeit im Lebensverlauf zu erhalten, wären Langzeitdaten erforderlich, die den gesamten Lebenszyklus des Menschen erfassen. Solche Daten sind jedoch nicht verfügbar. An ihrer Stelle können Querschnittsdaten zur Zeitnutzung nach Alterskohorten einigen Aufschluss darüber geben, wie die Freizeit in den verschiedenen Abschnitten des Lebenszyklus einer Person variieren kann. Es überrascht nicht, dass junge und insbesondere ältere Menschen mehr Zeit auf Freizeitaktivitäten verwenden als Personen im Erwerbsalter. In allen 18 in Tabelle 2.3 dargestellten OECD-Ländern verbringen Personen ab 65 Jahre im Durchschnitt durchweg mehr Zeit mit Freizeitaktivitäten als alle anderen Altersgruppen. Der Höchstwert für die Zeitanteile, die auf Freizeitaktivitäten verwendet werden, beläuft sich auf 39\% in Kanada, Norwegen und Polen. Der niedrigste Freizeitanteil bei über 65-Jährigen wird mit 25\% der gesamten Zeit in Mexiko verzeichnet.

Der Freizeitanteil der Bevölkerung im Alter von 15 bis 24 Jahren ist im Allgemeinen höher als der der Kohorten im Erwerbsalter. Wie vorherzusehen war, haben junge Menschen stets mehr Freizeit als Personen der Altersgruppe 25-44 Jahre. Etwas überraschender ist vielleicht, dass die Unterschiede im Vergleich zur Altersgruppe 4564 Jahre wesentlich geringer sind, obgleich die wenigsten bzw. keine Personen dieser höheren Altersgruppe bereits das gesetzliche Rentenalter erreicht haben. Die geringere Präsenz von kleinen Kindern in den Haushalten der älteren Gruppe im Erwerbsalter dürfte ein wichtiger Faktor für den Zugewinn an Freizeit gegenüber der jüngeren Gruppe im Erwerbsalter sein. Finnland, Italien und Neuseeland stechen durch hohe Freizeitanteile bei jungen Menschen hervor, die im Durchschnitt 30\% der Zeit oder mehr am Tag mit Freizeitaktivitäten verbringen. Der niedrigste Freizeitanteil bei jungen Menschen 9 Prozentpunkte unter dem Durchschnitt der OECD18 - ist in Mexiko zu finden.

Die 25- bis 44-Jährigen verfügen in jenen Ländern über mehr Freizeit, bei denen sich argumentieren ließe, dass gezielte Politikmaßnahmen den Anstoß zu einem ausgeglicheneren Ansatz gegenüber dem Berufsleben gegeben haben, bzw. in denen die Grenzsteuersätze sehr hoch sind: Norwegen (24\%), Finnland, Belgien und Deutschland (bei allen dreien 23\%) sind in dieser Altersgruppe führend (Parnanen et al., 2005). 


\section{Tabelle 2.3. Junge und ältere Menschen verfügen über mehr breit abgegrenzte} Freizeit als die Bevölkerung im Erwerbsalter

Freizeitanteile der Menschen nach Alter, prozentuale Anteile an der Gesamtzeit je Tag

\begin{tabular}{lcccc}
\hline & $15-24$ Jahre & $25-44$ Jahre & 45-64 Jahre & 65 Jahre und älter \\
\hline Australien & 27 & 17 & 22 & 34 \\
Belgien & 28 & 23 & 29 & 38 \\
Deutschland & 29 & 23 & 29 & 37 \\
Finnland & 30 & 23 & 27 & 38 \\
Frankreich & 27 & 22 & 25 & 37 \\
Italien & 30 & 21 & 25 & 37 \\
Japan & 21 & 16 & 19 & 34 \\
Kanada & 27 & 18 & 23 & 39 \\
Korea & 24 & 22 & 25 & 33 \\
Mexiko & 18 & 11 & 16 & 25 \\
Neuseeland & 30 & 20 & 22 & 35 \\
Norwegen & 29 & 28 & 39 \\
Polen & 28 & 22 & 26 & 39 \\
Schweden & 29 & 25 & 35 \\
Spanien & 29 & 21 & 26 &.. \\
Türkei & 28 &.. &.. & 36 \\
Ver. Königreich &.. & 22 & 26 & 37 \\
Ver. Staaten & 27 & 20 & 23 & $\mathbf{3 6}$ \\
OECD18 & 27 & $\mathbf{2 0}$ & $\mathbf{2 5}$ & \\
\hline
\end{tabular}

Anmerkung: In der Tabelle werden die Anteile der breit abgegrenzten Freizeit angegeben, die sich aus der Festsetzung des norwegischen Niveaus der persönlichen Pflege als Mindestniveau und der Einstufung der darüber hinausgehenden Anteile für die persönliche Pflege als Freizeit ergeben.

Quelle: Schätzungen des OECD-Sekretariats auf der Grundlage nationaler und multinationaler Erhebungen zur Zeitnutzung (2006, sofern verfügbar).

StatLink काजम $h t t p: / / d x . d o i . o r g / 10.1787 / 551073760502$

\section{Arten von Freizeitaktivitåten}

Welche Freizeitaktivitäten erfreuen sich besonderer Beliebtheit? Bestehen große Unterschiede bei den Freizeitaktivitäten, mit denen die Menschen in den einzelnen OECDLändern ihre Zeit verbringen? In Tabelle 2.4 ist die Freizeit nach fünf Hauptkategorien aufgeschlüsselt dargestellt: Multimedia-Unterhaltung zu Hause (Fernsehen oder Radiohören zu Hause), sonstige Freizeitaktivitäten (verschiedene Hobbys, Internetnutzung, Telefonate usw.), Freunde besuchen und/oder empfangen (sowohl zu Hause als auch im öffentlichen Raum), Teilnahme an bzw. Besuch von gesellschaftlichen Ereignissen (z.B. Konzerte, Kino, Museen usw.) und Sport (aktive Teilnahme an regelmäßigen körperlichen Aktivitäten, einzeln oder organisiert).

Im Durchschnitt der OECD18 stellt Fernsehen bzw. Radiohören knapp die beliebteste Freizeitaktivität dar, die 40\% der Zeit in Anspruch nimmt. Der Fernsehkonsum erreicht seinen Höchstwert mit 48\% in Mexiko, während sich sein Anteil am anderen Ende des Spektrums in Neuseeland hingegen auf lediglich $25 \%$ beläuft.

Die „sonstigen Freizeitaktivitäten“ sind im Durchschnitt ebenfalls sehr beliebt. Diese Beliebtheit ist z.T. auf die Tatsache zurückzuführen, dass es sich um eine breite Sammelkategorie handelt, die Hobbys, Computerspiele, Internetnutzung zu Freizeitzwecken, Telefonate, Kunst- und Handarbeiten, Haustiere ausführen usw. umfasst. Die sonstigen Aktivitäten nehmen in Italien bis zu 48\% der Freizeit ein, in der Türkei beträgt dieser Wert hingegen lediglich 25\%. Angesichts des Umfangs dieser Kategorie wäre es von großem Interesse gewesen, die Kategorie „sonstige Freizeitaktivitäten“ nach wichtigen 


\section{Tabelle 2.4. Fernsehen ist in allen betrachteten OECD-Ländern die beliebteste Freizeitbeschäftigung}

Prävalenz der verschiedenen Freizeitaktivitäten, prozentuale Anteile an der Freizeit insgesamt

\begin{tabular}{|c|c|c|c|c|c|}
\hline & $\begin{array}{c}\text { Fernsehen/Radiohören } \\
\text { zu Hause }\end{array}$ & $\begin{array}{l}\text { Sonstige Freizeit- } \\
\text { aktivitäten }\end{array}$ & $\begin{array}{l}\text { Freunde besuchen/ } \\
\text { empfangen }\end{array}$ & $\begin{array}{l}\text { Teilnahme an/Besuch } \\
\text { von Veranstaltungen }\end{array}$ & Sport \\
\hline Australien & 41 & 47 & 3 & 2 & 6 \\
\hline Belgien & 36 & 42 & 8 & 8 & 5 \\
\hline Deutschland & 28 & 46 & 4 & 15 & 7 \\
\hline Finnland & 37 & 40 & 7 & 8 & 8 \\
\hline Frankreich & 34 & 45 & 6 & 7 & 8 \\
\hline Italien & 28 & 48 & 6 & 10 & 8 \\
\hline Japan & 47 & 42 & 4 & 0 & 6 \\
\hline Kanada & 34 & 34 & 21 & 2 & 8 \\
\hline Korea & 35 & 41 & 16 & 1 & 7 \\
\hline Mexiko & 48 & 33 & 10 & 4 & 5 \\
\hline Neuseeland & 25 & 45 & 24 & 2 & 5 \\
\hline Norwegen & 31 & 33 & 14 & 15 & 8 \\
\hline Polen & 41 & 38 & 6 & 8 & 6 \\
\hline Schweden & 31 & 42 & 7 & 11 & 8 \\
\hline Spanien & 31 & 41 & 4 & 12 & 12 \\
\hline Türkei & 40 & 25 & 34 & 0 & 2 \\
\hline Ver. Königreich & 41 & 39 & 7 & 10 & 4 \\
\hline Ver. Staaten & 44 & 32 & 16 & 2 & 5 \\
\hline OECD18 & 36 & 40 & 11 & 6 & 7 \\
\hline
\end{tabular}

Quelle: Schätzungen des OECD-Sekretariats auf der Grundlage nationaler und multinationaler Erhebungen zur Zeitnutzung (2006, sofern verfügbar). Es ist hervorzuheben, dass Schlussfolgerungen auf Grund dieser Zahlen mit Vorsicht zu ziehen sind: Die methodischen Ansätze der nationalen Erhebungen zur Zeitnutzung unterscheiden sich in Bezug auf die Art und Weise, wie sie die Messgröße der sekundären Aktivitäten einbeziehen bzw. ausschließen. StatLink काज्ञा http://dx.doi.org/10.1787/551081652177

Unterkategorien aufzuschlüsseln. Leider war diese Aufschlüsselung auf Grund unzureichender Konsistenz im Ländervergleich in Bezug auf die Definitionen wichtiger Unterkategorien nicht möglich.

Bei der Kategorie „Freunde besuchen und/oder empfangen“, deren Anteile sich zwischen $34 \%$ in der Türkei und 3\% in Australien bewegen, gibt es zwischen den einzelnen Ländern sehr große Unterschiede 5 . „Aktivere“ Arten der Freizeitbeschäftigungen wie der Besuch kultureller Veranstaltungen und die Teilnahme an Sportaktivitäten sind in allen untersuchten OECD-Ländern weitaus weniger verbreitet. Der Besuch bzw. die Organisation kultureller Veranstaltungen ist in Deutschland und Norwegen relativ verbreitet, während dies in Japan, Korea und der Türkei weitaus seltener der Fall ist. Dasselbe ließe sich über Sportaktivitäten sagen, mit denen die Menschen in Spanien 12\% ihrer Freizeit verbringen, während der Zeitanteil in Belgien, Mexiko, Neuseeland und den Vereinigten Staaten lediglich $5 \%$ beträgt.

\section{Zufriedenheit mit der Zeit, die auf die verschiedenen Aktivitåten verwendet wird}

Die verschiedenen vorstehend aufgeführten Aktivitäten bieten zwar umfangreiche Einblicke in die Art und Weise, in der die Menschen ihre Zeit verbringen, erlauben jedoch keine unmittelbaren Rückschlüsse auf die Zufriedenheit, die sie auf Grund der Durchführung der verschiedenen Aktivitäten empfinden (entsprechend dem in der Einleitung zu diesem Kapitel erwähnten Ansatz des „Gemütszustands“ in Bezug auf die 
Freizeit). Für die Untersuchung der Beziehung zwischen Wohlergehen und Allokation von Zeit auf die verschiedenen Freizeitaktivitäten ist die Kombination von Daten aus zwei Quellen erforderlich: auf der einen Seite Daten aus Erhebungen zur Zeitnutzung, auf der anderen solche aus Erhebungen zur Zufriedenheit (Krueger et al., 2008). In diesen Erhebungen stufen die Befragten ihre Zufriedenheit nach der Ausführung bestimmter Aktivitäten anhand verschiedener Bewertungskriterien ein. In Abbildung 2.9 werden die Unterschiede bei der Rangfolge der Aktivitäten veranschaulicht, je nachdem ob die Befragten aufgefordert sind, eine objektive Bewertung einer ausgeführten Aktivität

\section{Abbildung 2.12. Freizeitbezogene Aktivitäten werden als angenehmer empfunden als arbeitsbezogene Aktivitäten (Ver. Staaten)}

Rangfolge der Aktivitäten in absteigender Reihenfolge der in der Momentaufnahme im Durchschnitt empfundenen Zufriedenheit

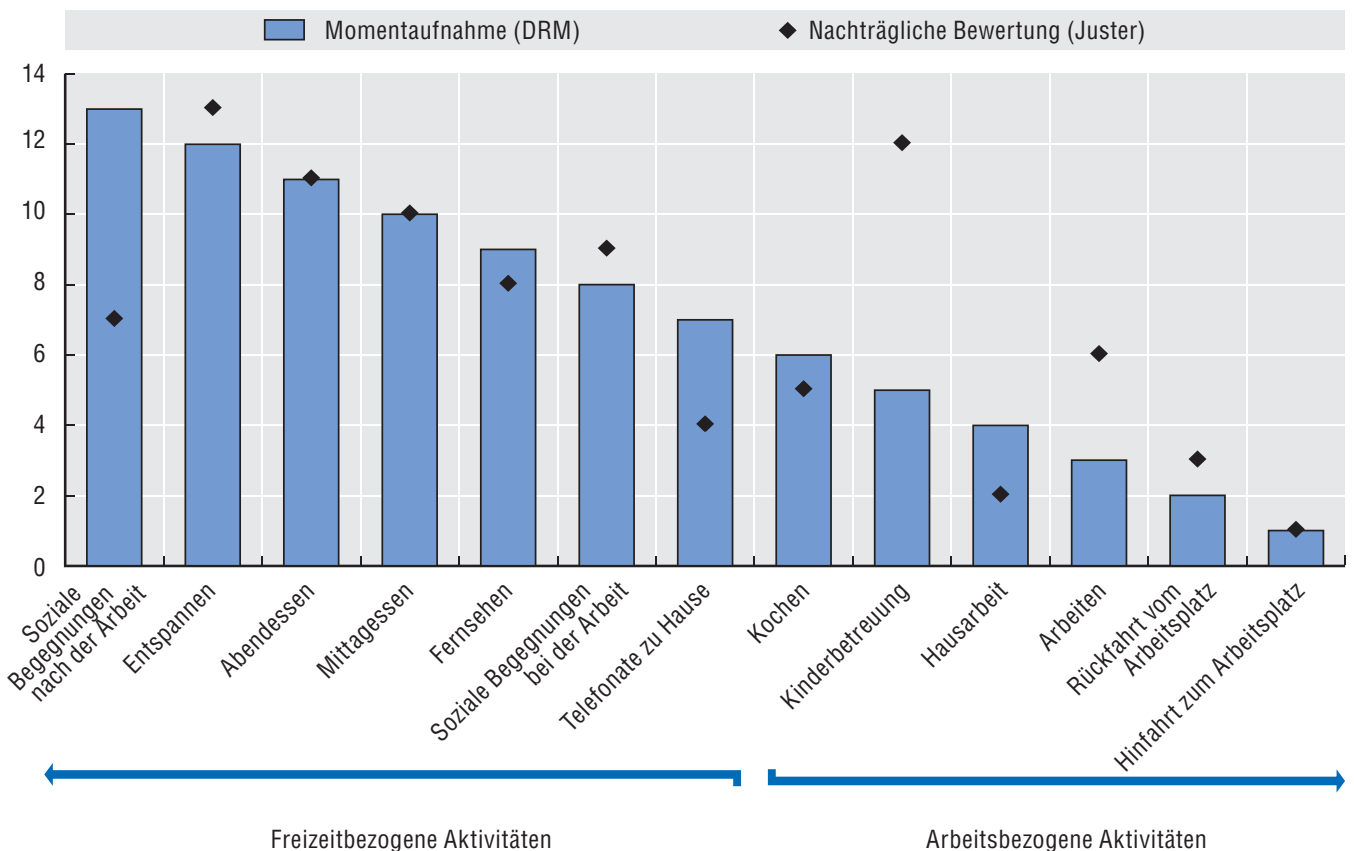

Anmerkung: Der obige Ansatz stützt sich auf Justers (1985, S. 333) wegweisende Beobachtung, dass „eine wichtige Zutat für die Erzeugung und Verteilung von Wohlergehen in dem Grad der Zufriedenheit besteht, die durch die Aktivitäten selbst hervorgerufen wird“. Zur Beurteilung der durch verschiedene Aktivitäten hervorgerufenen Zufriedenheit forderte Juster die Teilnehmer an seiner Erhebung auf, auf einer Skala von 0 bis 10 anzugeben, wie angenehm sie eine bestimmte Art von Aktivität im Allgemeinen empfanden, etwa ihre Arbeit oder die Betreuung ihrer Kinder. Spätere Forschungsarbeiten ergaben, dass solche allgemeinen Zufriedenheitsrangfolgen stark und auf theoretisch signifikante Weise von unmittelbaren Bewertungen abweichen können, die sich auf einzelne Erfahrungen der jeweiligen Aktivität beziehen. Um dieses Problem zu überwinden, verwenden Krueger et al. eine Zeittagebuch-Methode, die in größerem Zusammenhang mit den erinnerten emotionalen Erfahrungen der tatsächlichen Ereignisse und Umstände eines Tages steht, die Tagesrekonstruktionsmethode (Day Reconstruction Method, DRM). Die DRM ist ein Papier-und-Bleistift-Fragebogen, in dem zunächst Zeittagebuchdaten über den Vortag bei den Teilnehmern abgefragt werden. Für jede notierte Episode geben die Befragten die Art der Aktivität an, wer anwesend war und in welchem Ausmaß sie diverse Emotionen verspürten bzw. nicht verspürten. Die Befragten beschreiben ihren emotionalen Zustand während jeder Episode in Form von Intensitätseinstufungen verschiedener Dimensionen von Gefühlen, von denen einige positiv sind (z.B. „Zufrieden“, „Amüsiere mich“, „Freundlich“) und andere negativ (z.B. „Deprimiert“, „Ärgerlich“, „Frustriert“). Somit kombiniert die DRM Elemente von Erfahrungsstichproben und Zeittagebüchern und ist speziell darauf ausgelegt, die korrekte Erinnerung an Emotionen zu erleichtern.

Quelle: OECD-Berechnungen auf Grund von Daten aus Krueger et al. (2008). 
abzugeben („nachträgliche Bewertung“) oder ihre subjektiven Empfindungen zu beschreiben, während sie noch mit der Ausführung dieser bestimmten Aktivität beschäftigt sind („Momentaufnahme“). Sowohl die Daten als auch die Erhebung beziehen sich auf die Vereinigten Staaten, so dass unklar ist, in welchem Maße die anderen OECDLänder ähnlichen Mustern folgen.

Auf Grund der obigen Messgrößen ist klar ersichtlich, dass bestimmte Aktivitäten wie Entspannen und soziale Begegnungen nach der Arbeit als wesentlich angenehmer empfunden werden als etwa das Berufspendeln. Es ist nicht überraschend, dass in höherem Maße freizeitbezogene Aktivitäten wie Fernsehen, das Verzehren von Mahlzeiten (obgleich Mahlzeiten beim Zeitnutzungsansatz als persönliche Pflege eingestuft sind), Entspannen und soziale Begegnungen mit Kollegen in der Momentaufnahme durchgängig als sehr angenehm erfasst werden. Umgekehrt rangieren alle Aktivitäten, die unmittelbar oder mittelbar mit Arbeits- und Familienpflichten im Zusammenhang stehen, auf der Zufriedenheitsskala in der Momentaufnahme auf sehr niedrigen Plätzen.

Bei den meisten Aktivitäten bestehen im Vergleich zu den nachträglichen Bewertungen durch die Befragten lediglich geringe Unterschiede bei den Rangfolgen. Eine deutliche Ausnahme bildet in diesem Zusammenhang die Kinderbetreuung, die rückwirkend als angenehmer empfunden wird als in der Momentaufnahme. Arbeit weist ähnliche, aber weniger stark ausgeprägte Merkmale auf. Bei einigen Aktivitäten, etwa sozialen Begegnungen nach der Arbeit oder Hausarbeit, sind ebenfalls große Diskrepanzen vorhanden. Allerdings werden diese in der Momentaufnahme als angenehmer empfunden als rückwirkend in der nachträglichen Bewertung.

\section{Freizeit im Vergleich zu Messgrößen der Lebenszufriedenheit und des Markteinkommens}

Ein weiterer interessanter Aspekt der Freizeit ist der Umfang, in dem Freizeit mit anderen Messgrößen des Wohlergehens auf Länderebene korreliert. Zur Beantwortung dieser Frage werden zwei Hilfsindikatoren des globalen Wohlergehens mit zwei Messgrößen der Freizeit verglichen. Die zwei gewählten Messgrößen des Wohlergehens sind eine traditionelle Messgröße des Markteinkommens (in diesem Fall das Pro-KopfNettonationaleinkommen) und eine subjektive Messgröße des Wohlergehens (die Daten zur Lebenszufriedenheit aus der Erhebung Gallup World Poll 2006). Die zwei betrachteten Hauptmessgrößen der Freizeit sind die einfach durch Abzug der jährlich geleisteten Arbeitsstunden von der jährlichen Gesamtstundenzahl berechnete Restgröße und die breit abgegrenzte Messgröße der Zeitnutzung.

Aus Abbildung 2.13 und 2.14 geht hervor, dass das Niveau der Lebenszufriedenheit auf Länderebene durchaus positiv mit Freizeit korreliert ist, sowohl im Hinblick auf die Restgröße als auch auf die tatsächliche Freizeit. Zu den Ländern, in denen die Lebenszufriedenheit in Anbetracht ihres Niveaus der Restgröße der Freizeit erheblich niedriger ist, zählen Ungarn, Portugal und die Slowakische Republik. Auf der anderen Seite schneiden die Vereinigten Staaten und Australien angesichts ihres jeweiligen Freizeitniveaus bemerkenswert gut ab. In Bezug auf die Messgröße der Freizeit auf der Grundlage der Erhebungen zur Zeitnutzung scheinen die Australier erneut trotz relativ geringer Freizeitanteile zufrieden mit ihrem Leben zu sein. In Anbetracht ihrer Messgröße der Freizeit auf der Grundlage der Zeitnutzung ist das Niveau der Lebenszufriedenheit in Polen, der Türkei und Korea besonders niedrig. 


\section{Abbildung 2.13. Die Restgröße der Erwerbsarbeitszeit ist positiv mit der Lebenszufriedenheit korreliert}

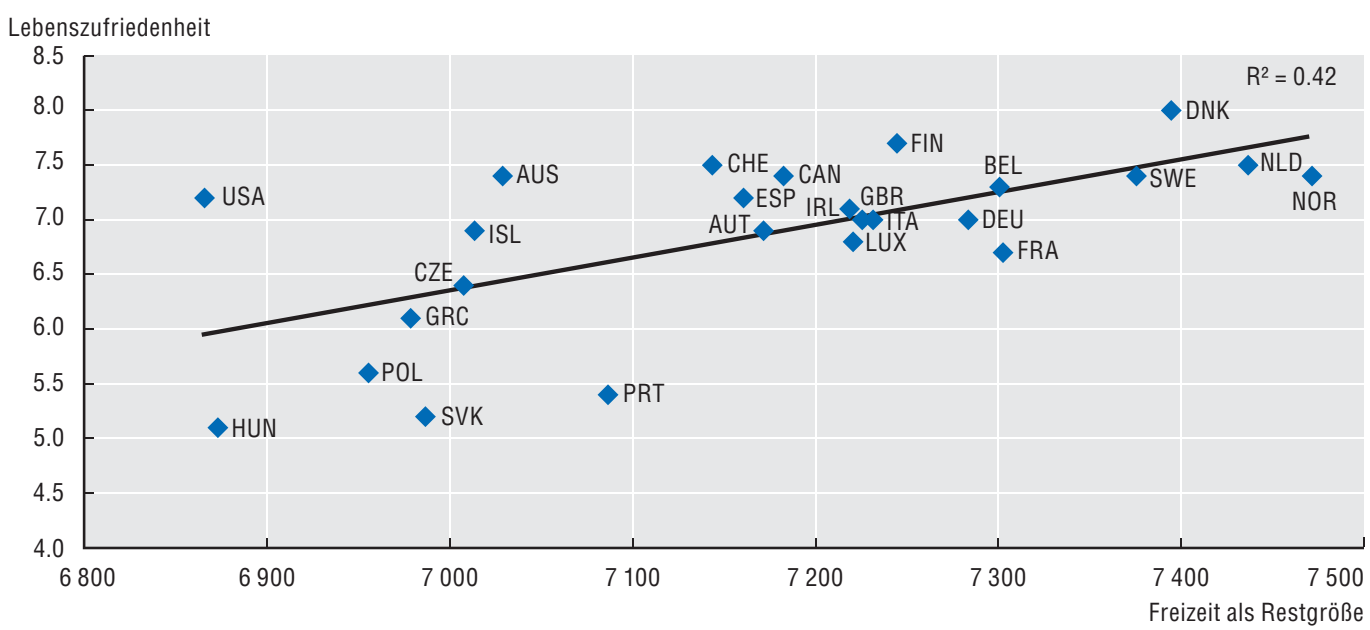

Quelle: Daten aus der Erhebung Gallup Life-satisfaction Survey 2006 und andere OECD-Daten. Schätzungen des OECD-Sekretariats auf der Grundlage der Ergebnisse der Europäischen Arbeitskräfteerhebungen und EIRO (2006, sofern verfügbar).

StatLink Aits $h t t p: / / d x . d o i . o r g / 10.1787 / 548761055333$

\section{Abbildung 2.14. Die breit abgegrenzte Freizeit ist positiv mit der Lebenszufriedenheit korreliert}

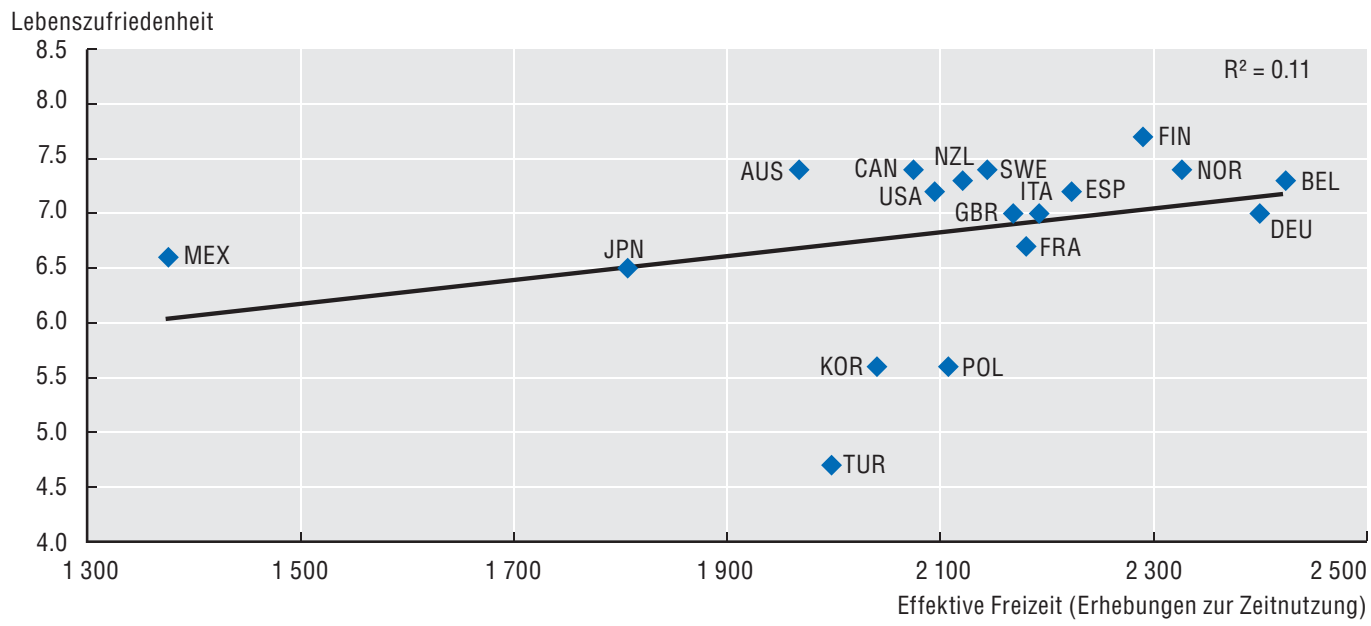

Quelle: Daten aus der Erhebung Gallup Life-satisfaction Survey 2006 und andere OECD-Daten. Schätzungen des OECD-Sekretariats auf der Grundlage nationaler und multinationaler Erhebungen zur Zeitnutzung (2006, sofern verfügbar).

StatLink क्ञाSस http://dx.doi.org/10.1787/548770544734

In Bezug auf die traditionelleren markteinkommensbasierten Messgrößen des Wohlergehens lässt sich aus Abbildung 2.15 und 2.16 die positive Korrelation zwischen Freizeit und der Höhe des Pro-Kopf-Nettonationaleinkommens ablesen. Die Korrelation ist wiederum sowohl in Bezug auf die Restgröße der Freizeit als auch auf die tatsächliche Freizeit positiv, was darauf hindeutet, dass Freizeit die Merkmale eines herkömmlichen Gutes besitzt: Mit zunehmendem Einkommen steigt die Nachfrage. 


\section{Abbildung 2.15. Die Restgröße der Erwerbsarbeitszeit ist positiv mit dem Pro-Kopf-NNE korreliert}

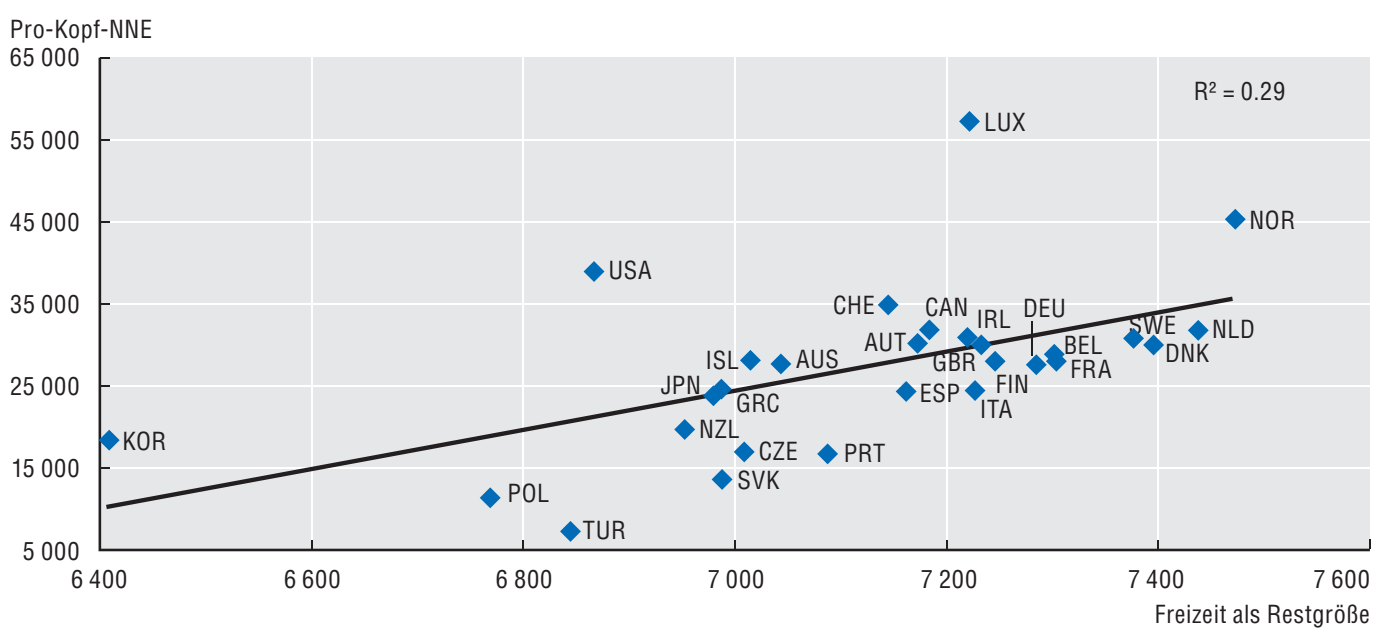

Anmerkung: Australien, Japan, Korea, Neuseeland, Polen und die Türkei verwenden Daten von 2005.

Quelle: Schätzungen des OECD-Sekretariats auf der Grundlage der OECD Annual National Accounts und Social Expenditure database (2006, sofern verfügbar), der Daten aus der Erhebung Gallup Life-satisfaction Survey 2006, der Ergebnisse der Europäischen Arbeitskräfteerhebungen und EIRO (2006, sofern verfügbar).

StatLink sils http://dx.doi.org/10.1787/548775647222

\section{Abbildung 2.16. Die breit abgegrenzte Freizeit ist positiv mit dem Pro-Kopf-NNE korreliert}

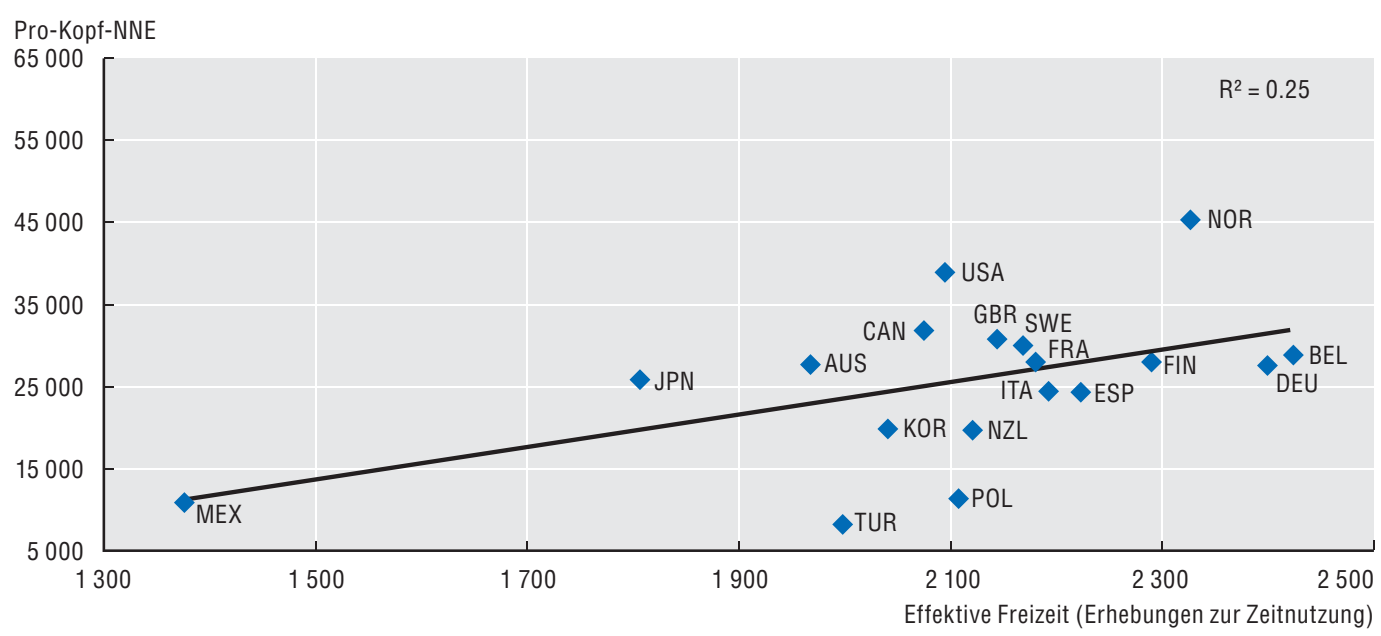

Anmerkung: Australien, Neuseeland und Polen verwenden Daten von 2005. NNE-Daten für Mexiko seit 2005 sind nicht verfügbar. Sie wurden für 2006 anhand des Wachstums des Pro-Kopf-BIP im Zeitraum 2004-06 geschätzt.

Quelle: Schätzungen des OECD-Sekretariats auf der Grundlage nationaler und multinationaler Erhebungen zur Zeitnutzung (2006, sofern verfügbar). OECD Annual National Accounts und Social Expenditure database (2006, sofern verfügbar). Schätzungen des OECD-Sekretariats auf der Grundlage der Ergebnisse der Europäischen Arbeitskräfteerhebungen und EIRO (2006, sofern verfügbar).

StatLink ants http://dx.doi.org/10.1787/548783364748

\section{Gesetzlicher Urlaubsanspruch und Freizeit}

Von größerem politischen Interesse ist die Beziehung zwischen gesetzlich geregeltem bezahlten Mindesturlaub sowie bezahlten Feiertagen und der Menge an Freizeit (als Restgröße oder abgeleitet aus Messgrößen der Zeitnutzung). Das vorrangige Ziel der gesetzlichen Regelung bezahlter Feiertage besteht darin, die verfügbare Freizeit nach 
Möglichkeit zu erhöhen und die Gesellschaft zu koordinieren, so dass Familien und Freunde ihre Freizeit leichter gemeinsam verbringen können. Im Hinblick auf den Umfang des gesetzlich festgelegten bezahlten Jahresurlaubs und die Anzahl der bezahlten gesetzlichen Feiertage bestehen zwischen den einzelnen OECD-Ländern erhebliche Unterschiede: von keinem einzigen Tag in den Vereinigten Staaten bis zu nahezu $10 \%$ des Jahres in Österreich, Portugal und Spanien (Abbildung 2.17) ${ }^{6}$.

\section{Abbildung 2.17. Bezahlter Jahresurlaub und bezahlte gesetzliche Feiertage im OECD-Raum}

Jahresurlaub und bezahlte gesetzliche Feiertage, Tage pro Jahr

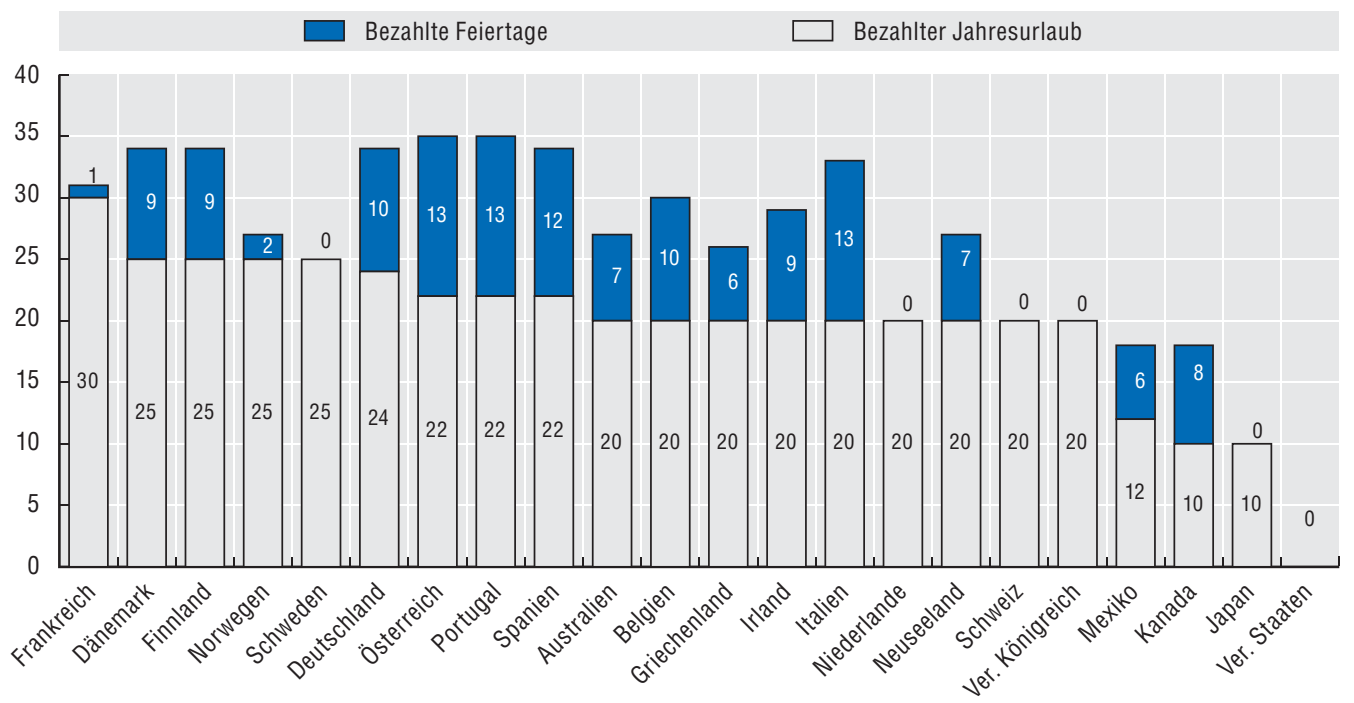

Anmerkung: In den Gesetzen mehrerer Staaten wird auf Werktage Bezug genommen, während sich andere auf Kalendertage oder Wochen beziehen. Für den Vergleich wurde eine Fünftagewoche zu Grunde gelegt. Die Vereinigten Staaten sind das einzige Land in der Gruppe, in dem die Arbeitgeber nicht gesetzlich verpflichtet sind, einen bezahlten Jahresurlaub zu gewähren. Naturgemäß gewähren allerdings viele Arbeitgeber in den in Abbildung 2.17 dargestellten Ländern auf der Grundlage von Tarif- und/oder individuellen Verträgen bezahlten Urlaub sowie gesetzliche Feiertage über das gesetzlich vorgeschriebene Mindestmaß hinaus. Dieser Faktor ist in den Vereinigten Staaten von besonderer Bedeutung, da im Gesetz keinerlei Mindestvorgaben für diese beiden Leistungen vorgesehen sind. Im Recht der Vereinigten Staaten gibt es keine Bestimmungen über bezahlte gesetzliche Feiertage, was auch für Japan, die Niederlande, Schweden und das Vereinigte Königreich gilt. Wegen weiterer Informationen vgl. Quellenangabe.

Quelle: Schmitt und Ray (2007), mit der Ausnahme Mexikos, dessen Zahlen vom OECD-Sekretariat erhoben wurden. StatLink क्ञाs http://dx.doi.org/10.1787/548802823410

Diese Politikdiskrepanzen können zwar die Unterschiede beim Umfang der Freizeit auf Länderebene erklären, es ist jedoch unklar, ob Personen in einem Land, in dem der Gesetzgeber ein gewisses Mindestmaß an bezahltem Jahresurlaub und/oder gesetzlichen Feiertagen garantiert, automatisch über mehr Freizeit verfügen. Abbildung 2.18 zeigt, dass der Umfang des gesamten Jahresurlaubs (bezahlter Jahresurlaub plus bezahlte Feiertage) und die Restgröße der Freizeit positiv korreliert sind, was darauf hindeutet, dass Politikmaßnahmen zur Regelung der Urlaubs- bzw. Feiertage wohl relativ erfolgreich sind. Darüber hinaus bleibt die positive Beziehung beim Vergleich des Gesamtjahresurlaubs mit den höherwertigen Messgrößen der Freizeit auf der Grundlage der Erhebungen zur Zeitnutzung in Abbildung 2.19 für die OECD18 bestehen, sie ist effektiv sogar leicht stärker. Die Regulierungspolitik im Bereich der bezahlten Feiertage kann den Umfang der Freizeit beeinflussen, über die die Menschen 
verfügen, obgleich offensichtliche Einschränkungen im Hinblick darauf bestehen, in der Korrelation notwendigerweise einen kausalen Effekt zu sehen.

\section{Abbildung 2.18. Es besteht eine relativ enge Beziehung zwischen der Restgröße der Erwerbsarbeitszeit und dem gesetzlich geregelten bezahlten Jahresurlaub}

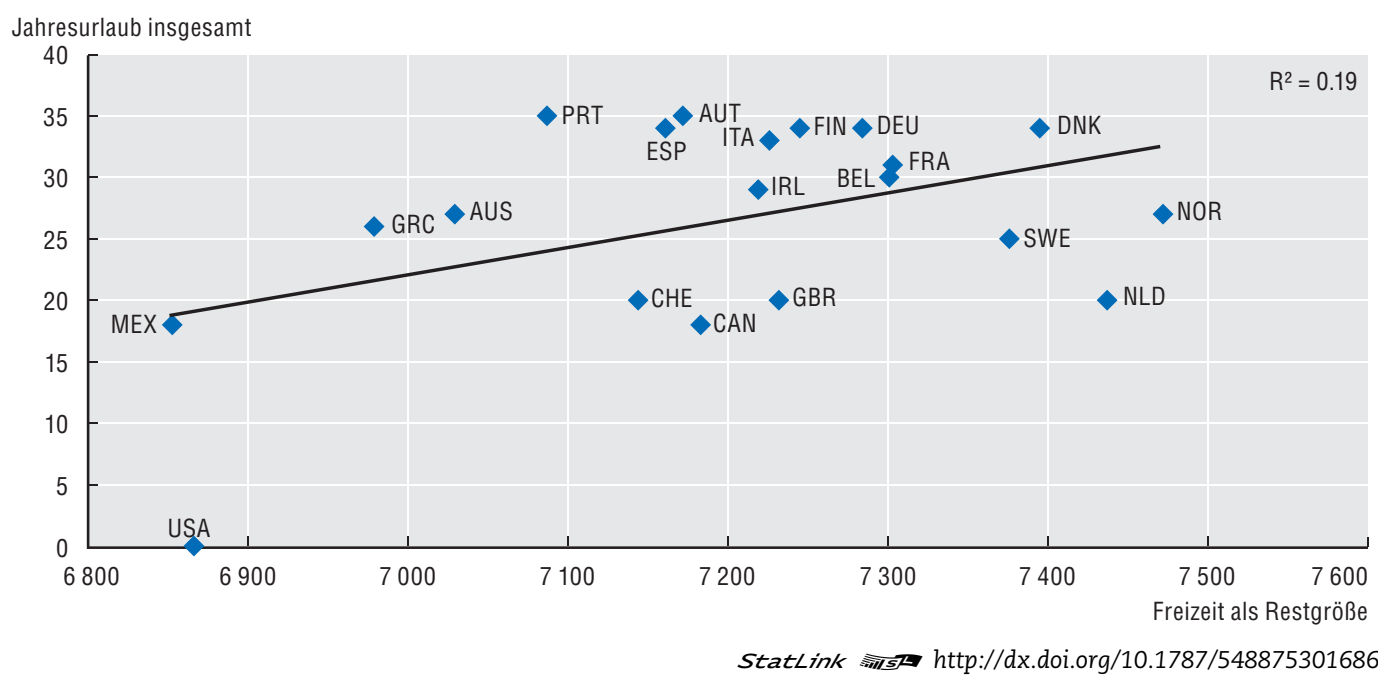

\section{Abbildung 2.19. Es besteht eine engere Beziehung zwischen der breit abgegrenzten Freizeit und dem gesetzlich geregelten bezahlten Jahresurlaub}

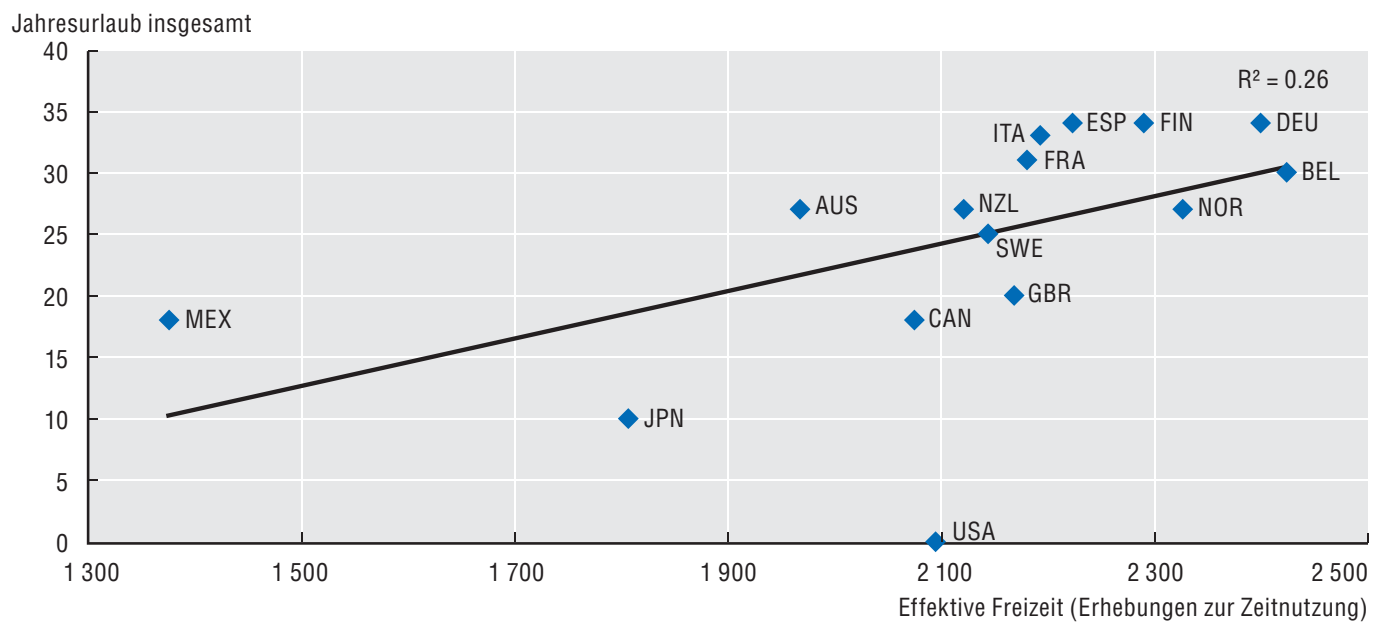

Anmerkung: In den Gesetzen mehrerer Staaten wird auf Werktage Bezug genommen, während sich andere auf Kalendertage oder Wochen beziehen. Für den Vergleich wurde eine Fünftagewoche zu Grunde gelegt.

Quelle: Schmitt und Ray (2007) sowie Schätzungen des OECD-Sekretariats auf der Grundlage nationaler und multinationaler Erhebungen zur Zeitnutzung (2006, sofern verfügbar).

StatLink क्ताsस http://dx.doi.org/10.1787/548881462071

\section{Schlussbetrachtungen}

In diesem Kapitel wurde untersucht, auf welche Art und Weise zwischen den einzelnen OECD-Ländern und innerhalb dieser Länder, ebenso wie im Zeitverlauf, Unterschiede im Hinblick auf die Freizeit bestehen. Dabei wurde den Schwierigkeiten bei der Begriffsdefinition im Zusammenhang mit der empirischen Messung der Freizeit Aufmerksamkeit geschenkt, wobei die Analyse eingangs anhand einer absichtlich 
vereinfachten Definition von Freizeit als „Zeit, in der nicht gearbeitet wird,“ erfolgte und dann schrittweise durch vergleichbare Daten aus Erhebungen zur Zeitnutzung bereichert wurde. Besonderes Augenmerk lag auf der Veränderlichkeit der Freizeitniveaus und -trends, je nach Verwendung unterschiedlicher Definitionen von „Freizeit“ (breiter und enger abgegrenzte Definition). Ein wichtiges Ergebnis dieses Kapitels ist, dass Begriffsdefinitionen bei der Analyse schwer fassbarer Gegenstände wie „Freizeit“ äußerst wichtig sind und die Gesamtländerrangfolgen sowie bestimmte soziodemografische Muster verändern können.

Die Daten aus den Erhebungen zur Zeitnutzung tragen zur Beobachtung der tatsächlichen Lebensbedingungen der OECD-Bevölkerungen bei. Mit Hilfe dieser Statistiken können die Lebensstile verschiedener Gruppen sowie ihre Entscheidungen für bestimmte Aktivitäten anstelle von anderen beobachtet und die Interpretation sowie das Verständnis verschiedener gesellschaftlicher und wirtschaftlicher Phänomene verbessert werden. Somit können sie den staatlichen Stellen von großem Nutzen sein, insbesondere denjenigen, die im Bereich der Politikberatung, -umsetzung und -überwachung tätig sind (Callister, 2004).

Die Politikverantwortlichen betrachten die Sozialpolitik in der Regel im Hinblick auf Effizienz und Gerechtigkeit. Freizeitbezogene Politikmaßnahmen sollten keine Ausnahme hiervon bilden. Die Politikentscheidungen beeinflussen die Freizeit gegenwärtig auf unmittelbare wie subtile Art und Weise. Der wichtigste Punkt ist, dass die Differenz zwischen Arbeit und Aktivitäten ohne Erwerbscharakter und damit das Maximum an Zeit, die für Freizeitaktivitäten zur Verfügung steht, vom Niveau des verfügbaren Einkommens (durch Einkommenseffekte) und den effektiven Grenzsteuersätzen (die sich auf die Substitution von Erwerbsarbeit durch Aktivitäten ohne Erwerbscharakter auswirken) beeinflusst wird. Generell ist die Palette von Politikmaßnahmen, die das Arbeitsangebot beeinflussen, vom Angebot an Kinder- und Schülerbetreuung bis zur öffentlichen Förderung der Hochschulen, für die Wahl zwischen Erwerbsarbeit und Aktivitäten ohne Erwerbscharakter von Bedeutung. Zusätzlich zum Abgaben- und Leistungssystem ist sowohl die Arbeitsmarkt- als auch die Produktmarktregulierung darauf ausgelegt, die verfügbare Menge an Freizeit in den OECD-Ländern zu beeinflussen. Im Hinblick auf den Arbeitsmarkt bestehen oftmals Regelungen für die gesetzlichen Feiertage und die Mindestdauer des Jahresurlaubs. Im Hinblick auf den Produktmarkt sind die Ladenöffnungszeiten und -tage ebenfalls reguliert, um die Freizeitmöglichkeiten der Menschen zu verbessern. Ob solche Politikmaßnahmen sich in der gewünschten Weise auf die Ziele auswirken, für die sie ergriffen wurden, ist nach wie vor ungeklärt.

Internationale Vergleiche der Freizeit anhand von Studien zur Zeitnutzung, die eine Vielzahl von Ländern abdecken, befinden sich noch in den Anfängen. In diesem Zusammenhang könnte eine Art von OECD-weitem Archiv für Erhebungen zur Zeitnutzung es den Forschern erlauben, ihre Vergleiche der Freizeit zwischen den einzelnen Mitgliedsländern und innerhalb dieser Länder zu verbessern. Dies könnte der naheliegendste nächste Schritt sein, um Freizeit im OECD-Vergleich besser zu verstehen, was die Mitgliedsländer, in denen derzeit noch keine solche Erhebung durchgeführt wird, dazu ermutigen könnte, die Beteiligung an regelmäßig durchgeführten international vergleichbaren Erhebungen zur Zeitnutzung in Erwägung zu ziehen. 


\section{Anmerkungen}

1. Ein Vergleich der Zeitnutzung nach Einkommensniveau wäre von großem Interesse gewesen. Die Standardisierung der Einkommensmessgrößen zwischen den einzelnen Ländern war jedoch nicht ausreichend, um den Versuch einer solchen Analyse zu unternehmen.

2. Vgl. auch Engler und Staubli (2008) wegen einer jüngeren und detaillierteren Analyse der Freizeit im Zeitverlauf, bei der Anpassungen im Hinblick auf die sich verändernde Altersstruktur, die Bildungsverteilung in der Bevölkerung sowie Veränderungen bei der Anzahl der Kinder vorgenommen wurden. Die Autoren verwenden Daten für dieselben fünf Länder wie die vorliegende Arbeit, allerdings über einen Zeitraum von 25 Jahren. Eine Hauptschlussfolgerung ihrer Studie ist, dass sich die Länder im Hinblick auf die Freizeit während des Untersuchungszeitraums aufeinander zubewegt haben.

3. Dieses Ergebnis widerspricht demjenigen von Burda et al. (2007), die hervorheben, dass die Geschlechter in den reichen Ländern in Bezug auf die Freizeit effektiv gleichgestellt seien. Es steht darüber hinaus auch zu einer anderen vor kurzem veröffentlichten Studie von Engler und Staubli (2008) im Widerspruch, in der geschlechtsspezifische Unterschiede im Hinblick auf die Freizeit ausgewiesen werden, die anhand von Erhebungen zur Zeitnutzung in Kanada, den Niederlanden, Norwegen, dem Vereinigten Königreich und den Vereinigten Staaten gemessen wurden. Die Autoren kommen zu dem Schluss, dass im Hinblick auf die wöchentliche Freizeit in allen dieser fünf Länder effektiv die Frauen im Vorteil sind. In ihrer Studie wird der Begriff „Freizeit“ zweifach definiert: Die erste Definition ist die Restzeit nach Abzug der Erwerbsarbeit (einschließlich der Zeit für das Berufspendeln) und unentgeltlicher Arbeit von der Gesamtzeit. Für die zweite Messgröße der Freizeit wird die Zeit für Ausbildung, das Erhalten persönlicher Dienstleistungen sowie für religiöse/Gemeinde-/freiwillige Aktivitäten abgezogen und die Zeit für Gartenarbeiten hinzugerechnet.

4. Engler und Staubli (2008) weisen für die Vereinigten Staaten im Vergleich zu anderen Ländern sowohl bei Männern als auch bei Frauen wesentlich höhere Einkaufszeiten aus (über zwei Stunden mehr pro Woche) als Burda et al. (2007).

5. Die Tatsache, dass das kulturell Australien nahestehende Neuseeland Freizeitanteile für das Besuchen von Freunden und Familie aufweist, die eher denen der Türkei nahekommen, deutet auf Probleme bei der Vergleichbarkeit der Daten zu den Arten der Freizeitaktivitäten hin.

6. Der Hauptunterschied zwischen gesetzlich vorgegebenem Jahresurlaub und gesetzlichen Feiertagen besteht darin, dass es beim Jahresurlaub im Allgemeinen einen gewissen zeitlichen Spielraum gibt, wann dieser genommen werden kann, während die Daten der gesetzlichen Feiertage in der Regel festgelegt sind. Darüber hinaus fallen die gesetzlichen Feiertage auf Grund des Kalenderzyklus gelegentlich auf Wochenenden und stellen in diesem Fall, wenigstens in einigen Ländern, keine arbeitsfreien Tage dar, wenn die Wochenendtage in der Regel arbeitsfrei sind.

\section{Literaturverzeichnis}

Aguiar, M. und E. Hurst (2006), „Measuring Trends in Leisure: The Allocation of Time Over FiveDecades“, National Bureau of Economic Research, Working Paper No. 12082, Cambridge MA, März.

Aliaga, C. und K. Winqvist (2003), „How Women and Men Spend their Time“, Statistics in Focus: Population und Social Conditions, Eurostat, Luxemburg.

Apps, P.F. (2003), „Gender, Time Use and Models of the Household“, IZA Discussion Paper No. 796, Forschungsinstitut zur Zukunft der Arbeit, Bonn, Juni.

Apps, P.F. und R. Rees (1996), „Labour Supply, Household Production and Intra-family Welfare Distribution“, Journal of Public Economics, Vol. 60, S. 199-209.

Apps, P.F. und R. Rees (1997), „Collective Labour Supply and Household Production“, Journal of Political Economy, Vol. 105, S. 178-190.

Apps, P.F. und R. Rees (2002), „Household Production, Full Consumption and the Costs of Children“, Labour Economics, Vol. 8, S. 621-648.

Biddle, J. und D. Hamermesh (1990), „Sleep and the Allocation of Time“, Journal of Political Economy, Vol. 98, No. 5, S. 922-943. 
Burda, M.C., D.S. Hamermesh und P. Weil (2007), „Total Work, Gender, and Social Norms“, IZA Discussion Paper No. 2705, Forschungsinstitut zur Zukunft der Arbeit, Bonn, März.

Callister, P. (2004), „Time-Use Data and Work-Life Policy Development“, Social Policy Journal of New Zealand, No. 22, Juli.

Causa, O. (2008), „Explaining Differences in Hours Worked among OECD Countries: An Empirical Analysis“, OECD Economics Department Working Papers, No. 596, OECD, Paris.

Chiappori, A. (1997), „Introducing Household Production in Collective Models of Labour Supply“, Journal of Political Economy, Vol. 105, No. 1, S. 191-209.

Engler, M. und S. Staubli (2008), „The Distribution of Leisure Time across Countries and Over Time“, Department of Economics Discussion Paper No. 2008-14, Universität St. Gallen, Schweiz.

Gronau, R. (1976), „Leisure, Home Production and Work - The Theory of the Allocation of Time Revisited“, NBER Working Paper No. 137, Cambridge, MA, Mai.

Koreman, P. und A. Kapteyn (1987), „A Disaggregated Analysis of the Allocation of Time within the Household“, Journal of Political Economy, Vol. 95, No. 2, S. 223-249.

Krueger, A.B., D. Kahneman und D. Schkade (2008), „National Time Accounting: The Currency of Life“, Dritter Entwurf eines unveröffentlichten Dokuments, 31. März.

Parnanen, A., H. Sutela und S. Mahler (2005), Combining Family and Full-Time Work, European Foundation for the Improvement of Living and Working Conditions, Report No. TN0510TR02, Dublin.

Ramey, V.A. und F. Neville (2006), „A Century of Work and Leisure“, NBER Working Paper No. 12264, Cambridge, MA, Mai.

Schmitt, J. und R. Ray (2007), No Vacation Nation USA - A Comparison of Leave and Holiday in OECD Countries, Center for Economic und Policy Research, European Economic and Employment Policy Brief No. 3-2007, Washington, Mai.

Solberg, E. und D. Wong (1992), „Family Time-use, Home Production, Market Work and Work-Related Travel“, Journal of Human Resources, Vol. 27, No. 3, S. 485-510.

Veblen, T. (1899), The Theory of the Leisure Class, Penguin Classics. 


\section{ANHANG 2.A1}

\section{Wesentliche Merkmale der Erhebungen zur Zeitnutzung}

In diesem Anhang werden einige Merkmale der Erhebungen zur Zeitnutzung beschrieben, die vom OECD-Sekretariat für die Berücksichtigung in diesem Kapitel für geeignet erachtet wurden.

\section{Kontext}

Erhebungen zur Zeitnutzung sind das vorrangige statistische Instrument zur Erfassung von Daten darüber, wie genau die Menschen ihre Zeit auf die verschiedenen Aktivitäten des Alltags aufteilen. Die Erhebungen bestehen hauptsächlich aus Tagebüchern, die von einer großen Anzahl von Personen über alle Aktivitäten während eines oder mehrerer für einen bestimmten Zeitraum repräsentativer Tage geführt werden. Die Erhebungsteilnehmer beschreiben die von ihnen ausgeführten Aktivitäten, die dann von den nationalen statistischen Ämtern in eine Reihe deskriptiver Kategorien umkodiert werden. Bei einer gut konzipierten Erhebung werden die Aktivitäten über einen Zeitraum von insgesamt 24 Stunden am Tag (bzw. 1440 Minuten) erfasst.

Das Interesse an Zeitnutzungserhebungen hat in den vergangenen zwanzig Jahren deutlich zugenommen. Eine Reihe nationaler statistischer Ämter hat in den letzten Jahrzehnten groß angelegte Erhebungen zur Zeitnutzung durchgeführt.

Die meisten Datensätze zur Zeitnutzung sind umfangreich genug, um daraus verlässliche Messgrößen der Zeitallokation über das ganze Jahr zu generieren, die Treffsicherheit dieser Schätzwerte variiert von einem Land zum anderen jedoch erheblich. Unterschiede bei den Erhebungsmerkmalen, der Anzahl der in der Stichprobe erfassten Tage, über die Tagebuch geführt wurde, und der gewählten Kategorisierung der Aktivitäten wirken sich u.U. auf die Vergleichbarkeit der Ergebnisse zwischen den einzelnen Ländern aus.

Im Folgenden werden die wichtigsten Dimensionen beschrieben, in denen sich die Erhebungen zur Zeitnutzung voneinander unterscheiden:

- Konzeption der Stichprobe. Alle in diesem Kapitel berücksichtigten Erhebungen zur Zeitnutzung beruhen auf Stichproben der gebietsansässigen Bevölkerung (ohne in Gemeinschaftsunterkünften, Heimen oder Anstalten lebende Personen), die für die jeweiligen Länder repräsentativ sind. Die nationalen Erhebungen unterscheiden sich jedoch im Hinblick auf die Konzeption der Stichprobe, wobei einige Erhebungen auf einer Zufallsstichprobe beruhen, andere hingegen eine festgelegte Stichprobe verwenden, die von anderen groß angelegten Bevölkerungserhebungen übernommen wurde. Die Erhebungen zur Zeitnutzung unterscheiden sich auch in Bezug auf den Stichprobenumfang (von rd. 4000 bis rd. 200000 Personen), das Alter der in die Stichprobe 
einbezogenen Befragten (im Allgemeinen Personen ab 15 Jahre, wobei es jedoch mehrere Ausnahmen gibt) und die Antwortquoten (auf Grund der hohen Nichtbeantwortungsquoten wird die tatsächliche Anzahl der fertiggestellten Zeitnutzungstagebücher bei manchen Erhebungen neu gewichtet, um potenziell nicht teilnehmenden Personen Rechnung zu tragen). Weitere Unterschiede zwischen den verschiedenen Erhebungen zur Zeitnutzung bestehen im Hinblick auf die erhobenen Daten über demografische Merkmale, die Art und Weise, wie diese Merkmale definiert werden (z.B. Erwerbsstatus), und in Bezug auf die Kontextinformationen, die für jede Aktivität angegeben werden (z.B. wo diese ausgeführt wurden, ob weitere Personen zu diesem Zeitpunkt anwesend waren usw.).

- Klassifizierung der Aktivitäten. Bei allen Erhebungen werden die durch die Befragten mündlich und/oder schriftlich abgegebenen Beschreibungen ihrer Aktivitäten einer Reihe breiter gefasster Kategorien zugeordnet. Da diese Kodierungssysteme entsprechend den Zielen und Ambitionen der jeweiligen Erhebung variieren, führen sie zu unterschiedlich detaillierten Klassifikationen*. Die Unterschiede bei der Kategorisierung ergeben sich hauptsächlich aus den hinsichtlich der Einteilung bestimmter Aktivitäten in weiter gefasste Kategorien getroffenen Entscheidungen. So werden z.B. in manchen Erhebungen alle Einkaufsaktivitäten in einer Kategorie „Einkäufe“ zusammengefasst, während in anderen nach dem Zweck der Einkäufe (z.B. Einkäufe von Lebensmitteln, Bürobedarf, Haushaltsgegenständen/-dienstleistungen usw.) differenziert wird. Bei einigen Erhebungen werden Sport und ehrenamtliche Tätigkeiten einer allgemeinen Kategorie "Soziale Begegnungen und Freizeit" zugeordnet, während in anderen zwischen allein ausgeführten Freizeitaktivitäten (Computerspielen) und gemeinsam ausgeführten Freizeitaktivitäten (Teilnahme an einem sportlichen Wettkampf) unterschieden wird. Bei einigen Erhebungen werden staatsbürgerliche und religiöse Aktivitäten den „Sonstigen Aktivitäten“ zugerechnet, während diese bei anderen keinerlei Berücksichtigung finden. Bei einigen Erhebungen wird auch die auf die Teilnahme an der Erhebung verwendete Zeit erfasst, während dies bei anderen nicht der Fall ist. Und schließlich umfassen einige Erhebungen eine separate Kategorie für Fahrzeiten, manchmal unterteilt nach dem Fahrzweck (d.h. die Fahrzeit zur Arbeit und zurück wird in der Kategorie „Arbeitsbezogene Aktivitäten“ berücksichtigt, und Fahrzeiten zu Urlaubszwecken werden der Kategorie „Soziale Begegnungen und Freizeit“ zugeordnet), während solche Fahrzeiten in anderen Erhebungen in die breiter gefasste Kategorie eingeordnet werden, die sie betreffen.

- Anzahl der Tage, an denen Tagebuch geführt wird. Im Hinblick auf die Anzahl der Tage, an denen jeder Teilnehmer Tagebuch führen soll, werden unterschiedliche methodische Entscheidungen getroffen. Beispielsweise sind die Teilnehmer bei der in den Vereinigten

* Die Erhebung American Time Use Survey (ATUS) beispielsweise beginnt mit einem sechsstelligen Kodierungssystem mit drei Gliederungsebenen, aus denen grundlegende Kodizes in 17 Oberkategorien aggregiert werden: 1. Aktivitäten im Bereich der persönlichen Pflege (hauptsächlich Schlaf), 2. Aktivitäten im Haushalt, 3. Pflege und Unterstützung von Haushaltsmitgliedern, 4. Pflege und Unterstützung von nicht dem Haushalt angehörigen Personen, 5. Erwerbsarbeit und arbeitsbezogene Aktivitäten, 6. Bildung, 7. Einkäufe von Verbrauchsgütern (z.B. Nahrungsmitteleinkäufe), 8. Entgegennahme von Dienstleistungen von Angehörigen der freien Berufe oder im Bereich der persönlichen Pflege gegen Entgelt (z.B. Arztbesuche), 9. Entgegennahme von Haushaltsdienstleistungen gegen Entgelt, 10. Entgegennahme von öffentlichen Dienstleistungen sowie staatsbürgerliche Pflichten, 11. Essen und Trinken, 12. Soziale Begegnungen, Entspannung und Freizeit, 13. Sport, Bewegung und Erholung, 14. Religiöse und spirituelle Aktivitäten, 15. Ehrenamtliche Aktivitäten, 16. Telefonate und 17. Reisen. 
Staaten durchgeführten Erhebung ATUS aufgefordert, das Zeittagebuch lediglich einen Tag lang zu führen, bei den meisten Erhebungen werden jedoch üblicherweise Daten über zwei Tage erhoben. Beide Möglichkeiten haben Vor- und Nachteile. Die Zeit, die an einem beliebigen Tag auf verschiedene Aktivitäten verwendet wird, ist u.U. nicht repräsentativ für die Art und Weise, wie die Befragten üblicherweise ihre Zeit verbringen, obgleich solche Anomalien sich über die gesamte Stichprobe der Befragten ausgleichen dürften. Umgekehrt ermöglichen Zeitnutzungsdaten $\mathrm{zu}$ mehreren Tagen die Behandlung von Fragen im Zusammenhang mit der Art und Weise, wie Aktivitäten über mehrere Tage miteinander kombiniert werden, obgleich der Preis hierfür niedrigere Antwortquoten sind. Welchen relativen Wert es hat, mehrere Zeittagebücher von jedem einzelnen Befragten anstelle jeweils eines Zeittagebuchs von einer größeren Anzahl von Befragten zu bekommen, ist in der Regel vom generellen Ziel der Erhebung abhängig.

- Zeitraum, in dem die Erhebung durchgeführt wird. Die Angaben zur Zeitnutzung sind im Allgemeinen repräsentativ für Aktivitäten, mit denen die Befragten an den Wochentagen beschäftigt sind, für die sie die Zeitnutzung erfassen. Die auf dieser Grundlage berechneten Schätzwerte können jedoch u.U. nicht für das ganze Jahr als repräsentativ gelten. Daher unterscheiden sich die Erhebungen zur Zeitnutzung in Bezug auf den Zeitraum, der von den einzelnen Erhebungen abgedeckt wird. Die in den Vereinigten Staaten durchgeführte Erhebung etwa wird über das ganze Jahr verteilt durchgeführt und liefert genaue Schätzwerte für das ganze Jahr. Andere Erhebungen decken bestimmte Zeiträume im Jahr ab, die in der Regel so gewählt werden, dass saisonal bedingte Verzerrungen auf Grund gesetzlicher Feiertage oder Jahresurlaub bei Arbeitskräften vermieden werden. Bei einigen Ländern kann der für die Feldarbeit gewählte Zeitraum jedoch u.U. nicht für das ganze Jahr als repräsentativ gelten. Die verschiedenen Entscheidungen im Hinblick auf den für die Feldarbeit gewählten Zeitraum sind in der Regel von den Zielen der Erhebung, den praktischen Möglichkeiten der statistischen Ämter und der Verfügbarkeit von Finanzmitteln abhängig.

- Erfassung sekundärer Aktivitäten. Die Erhebungen unterscheiden sich ebenfalls darin, wie und ob sie Aktivitäten erfassen, die gleichzeitig ausgeübt werden. Im Allgemeinen werden die Daten so kodiert, dass sie Personen jeweils bei der Ausübung einer einzigen Aktivität abbilden. In manchen Fällen umfassen die Erhebungen jedoch separate Fragen, mit denen Informationen über gleichzeitig erfolgende Aktivitäten gewonnen werden sollen (z.B. Fernsehen neben dem Kochen oder die Betreuung von Kindern neben anderen Beschäftigungen), was eine Unterscheidung zwischen „primären“ und „sekundären“ Aktivitäten ermöglicht. Selbst wenn Daten über gleichzeitig erfolgende Aktivitäten erhoben werden, stellen die meisten statistischen Ämter Einheitlichkeit bei der Kodierung der Beschreibung ihrer Hauptaktivitäten durch die Befragten sicher und erstellen dann detailliertere Basiskodizes für Unterkategorien. Eine Einschränkung in Bezug auf die auf diese Weise gewonnenen Daten ist, dass die „primären“ Aktivitäten sorgfältig aufgezeichnet werden, während die „sekundären“ in der Regel übersehen werden. Ein weiteres Element, das die Vergleichbarkeit der Schätzwerte für sekundäre Aktivitäten beeinträchtigt, ist die Frage, ob Aktivitäten, die im Allgemeinen nur wenige Minuten in Anspruch nehmen - etwa das Umfüllen einer Wäscheladung aus der Waschmaschine in den Wäschetrockner -, mit ausreichender Konsistenz angegeben werden, um vergleichbare Schätzwerte für die auf diese Aktivitäten verwendete Zeit zu gewinnen. Auf Grund der Nichtberücksichtigung sekundärer Aktivitäten wird die Zeit, 
die auf die einzelnen Aufgaben entfällt, die gleichzeitig mit anderen Aufgaben ausgeführt werden können, in der Regel untererfasst.

- Erfassung von Aktivitäten nach Ehepartnern. Die nationalen Erhebungen unterscheiden sich ebenfalls in Bezug auf den Umfang, in dem Daten über verschiedene Mitglieder desselben Haushalts erhoben werden. Während bei einigen Erhebungen Daten über jeweils eine Person je Haushalt erfasst werden, stützen sich andere (z.B. in Australien, Deutschland und Korea) auf Tagebücher, die bei verheirateten Paaren von beiden Ehepartnern ausgefüllt werden. Tagebücher von beiden Ehepartnern erhellen bis zu einem gewissen Grad bestimmte Wechselwirkungen zwischen der jeweiligen Zeitnutzung der Partner (z.B. im Hinblick auf die Zeit, die Eltern zusammengenommen auf die Betreuung ihrer Kinder verwenden), obgleich diese Daten zum Zweck der Messung der Zeitallokation der Bevölkerung irrelevant sind. Wie in anderen Fällen auch, müssen die Vorteile dieser zusätzlichen Daten gegen den potenziellen Preis in Bezug auf die Antwortquoten und die Datengenauigkeit abgewogen werden. 
Table 2.A1.1. Dokumentation der Methodiken nationaler Erhebungen zur Zeitnutzung

\begin{tabular}{|c|c|c|c|c|c|c|c|c|c|}
\hline & Name der Erhebung & Behörde & Jahr & $\begin{array}{l}\text { Website (Daten und } \\
\text { Dokumentation) }\end{array}$ & Erhebungszeitraum & Erfasste Bevölkerung & Stichprobenumfang & $\begin{array}{l}\text { Anzahl und Art } \\
\text { der Tagebuchtage }\end{array}$ & $\begin{array}{l}\text { Sonstige } \\
\text { Erhebungsmerkmale }\end{array}$ \\
\hline Australien & Time Use Survey & $\begin{array}{l}\text { Australian } \\
\text { Bureau of } \\
\text { Statistics }\end{array}$ & 2006 & $\begin{array}{l}\text { www.abs.gov.au/ } \\
\text { AUSSTATS/ } \\
\text { abs@.nsf/ } \\
\text { Latestproducts/ } \\
\text { 4153.0Main\%20Feat } \\
\text { ures22006?opendoc } \\
\text { ument\&tabname=Su } \\
\text { mmary\&prodno=415 } \\
\text { 3.0\&issue=2006\&nu } \\
\text { m=\&view }=\end{array}$ & $\begin{array}{l}\text { Vier Zeiträume von je } \\
13 \text { Tagen, die einen } \\
\text { repräsentativen Anteil } \\
\text { an gesetzlichen } \\
\text { Feiertagen und } \\
\text { Schulferien umfassen }\end{array}$ & $\begin{array}{l}\text { Personen ab } 15 \text { Jahre, wohnhaft in } \\
\text { privaten Wohneinheiten (ohne } \\
\text { Personen, die in sehr abgelegenen } \\
\text { und nicht privaten Wohneinheiten } \\
\text { leben, Haushalte, in denen } \\
\text { Personen nicht australischer } \\
\text { Staatsangehörigkeit leben, und } \\
\text { indigene Bevölkerungsgruppen) }\end{array}$ & Rd. 3900 Haushalte & $\begin{array}{l}\text { Tagebuch über zwei } \\
\text { separate Tage, mit } \\
\text { festgelegten Intervallen } \\
\text { von } 5 \text { Minuten }\end{array}$ & $\begin{array}{l}\text { Daten werden z.T. durch } \\
\text { Interviews und z.T. durch } \\
\text { selbst ausgefüllte } \\
\text { Tagebücher erhoben } \\
\text { Klassifizierung in primäre } \\
\text { und sekundäre Aktivitäten, } \\
\text { für wen die Aktivität } \\
\text { ausgeführt wird, wer } \\
\text { ansonsten anwesend ist und } \\
\text { wo die Aktivität stattfindet }\end{array}$ \\
\hline Belgien & $\begin{array}{l}\text { Harmonised } \\
\text { European Time Use } \\
\text { Survey (HETUS) }\end{array}$ & $\begin{array}{l}\text { Eurostat und } \\
\text { nationales } \\
\text { statistisches } \\
\text { Amt }\end{array}$ & 2005 & $\begin{array}{l}\text { www.testh2.scb.se/ } \\
\text { tus/tus/ }\end{array}$ & Ein Jahr & $\begin{array}{l}\text { Zwei Erhebungspopulationen } \\
\text { werden betrachtet: } 1 \text {. Personen ab } \\
12 \text { Jahre, die } 2005 \text { der belgischen } \\
\text { Bevölkerung angehörten. In } \\
\text { Gemeinschaftsunterkünften, } \\
\text { Heimen oder Anstalten lebende } \\
\text { Personen wurden nicht als Teil der } \\
\text { Erhebungspopulation betrachtet. } \\
\text { 2. Haushalte: private Haushalte } \\
\text { (jeweils einschl. aller darin } \\
\text { lebenden Personen). } \\
\text { Entsprechend den beiden } \\
\text { vorstehend definierten } \\
\text { Erhebungspopulationen werden } \\
\text { zwei Rahmen vom Nationalregister } \\
\text { der natürlichen Personen } \\
\text { abgeleitet. }\end{array}$ & $\begin{array}{l}35000 \text { Haushalte in } \\
\text { der ursprünglichen } \\
\text { Stichprobe (vor } \\
\text { Nichtbeantwortung) }\end{array}$ & $\begin{array}{l}\text { Jeder Befragte füllt } \\
\text { Tagebücher für zwei } \\
\text { Tagebuchtage aus, die } \\
\text { jeweils } 24 \text { Stunden } \\
\text { abdecken }\end{array}$ & $\begin{array}{l}\text { Kurze, willkürliche Momente } \\
\text { im Leben der Menschen } \\
\text { werden beobachtet, die daher } \\
\text { nicht als repräsentativ } \\
\text { betrachtet werden können }\end{array}$ \\
\hline Deutschland & $\begin{array}{l}\text { Harmonised } \\
\text { European Time Use } \\
\text { Survey (HETUS) }\end{array}$ & $\begin{array}{l}\text { Eurostat und } \\
\text { nationales } \\
\text { statistisches } \\
\text { Amt }\end{array}$ & 2002 & $\begin{array}{l}\text { www.testh2.scb.se/ } \\
\text { tus/tus/ }\end{array}$ & Ein Jahr & $\begin{array}{l}\text { Alle privaten Haushalte mit } \\
\text { Personen ab } 10 \text { Jahre, ohne } \\
\text { Personen ohne festen Wohnsitz } \\
\text { und Personen in Gemeinschafts- } \\
\text { unterkünften und ähnlichen } \\
\text { Einrichtungen (Kasernen, } \\
\text { Altersheime usw.) }\end{array}$ & $\begin{array}{l}\text { Rd. } 5443 \text { Haushalte } \\
\text { in der endgültigen } \\
\text { Stichprobe }\end{array}$ & $\begin{array}{l}\text { Jeder Befragte füllt } \\
\text { Tagebücher für zwei } \\
\text { Tagebuchtage aus, die } \\
\text { jeweils } 24 \text { Stunden } \\
\text { abdecken }\end{array}$ & $\begin{array}{l}\text { Kurze, willkürliche Momente } \\
\text { im Leben der Menschen } \\
\text { werden beobachtet, die daher } \\
\text { nicht als repräsentativ } \\
\text { betrachtet werden können }\end{array}$ \\
\hline Finnland & $\begin{array}{l}\text { Harmonised } \\
\text { European Time Use } \\
\text { Survey (HETUS) }\end{array}$ & $\begin{array}{l}\text { Eurostat und } \\
\text { nationales } \\
\text { statistisches } \\
\text { Amt }\end{array}$ & 1998 & $\begin{array}{l}\text { www.testh2.scb.se/ } \\
\text { tus/tus/ }\end{array}$ & Ein Jahr & $\begin{array}{l}\text { Personen ab } 10 \text { Jahre, die in } \\
\text { privaten Haushalten leben, und alle } \\
\text { Haushaltsmitglieder }\end{array}$ & $\begin{array}{l}4800 \text { Haushalte mit } \\
12512 \text { Personen, } \\
\text { darunter } 10978 \text { über } \\
10 \text { Jahre }\end{array}$ & $\begin{array}{l}\text { Jeder Befragte füllt } \\
\text { Tagebücher für zwei } \\
\text { Tagebuchtage aus, die } \\
\text { jeweils } 24 \text { Stunden } \\
\text { abdecken }\end{array}$ & $\begin{array}{l}\text { Kurze, willkürliche Momente } \\
\text { im Leben der Menschen } \\
\text { werden beobachtet, die daher } \\
\text { nicht als repräsentativ } \\
\text { betrachtet werden können }\end{array}$ \\
\hline
\end{tabular}


Table 2.A1.1. Dokumentation der Methodiken nationaler Erhebungen zur Zeitnutzung (Forts.)

\begin{tabular}{|c|c|c|c|c|c|c|c|c|c|}
\hline & Name der Erhebung & Behörde & Jahr & $\begin{array}{l}\text { Website (Daten und } \\
\text { Dokumentation) }\end{array}$ & Erhebungszeitraum & Erfasste Bevölkerung & Stichprobenumfang & $\begin{array}{l}\text { Anzahl und Art } \\
\text { der Tagebuchtage }\end{array}$ & $\begin{array}{l}\text { Sonstige } \\
\text { Erhebungsmerkmale }\end{array}$ \\
\hline Frankreich & $\begin{array}{l}\text { Harmonised } \\
\text { European Time Use } \\
\text { Survey (HETUS) }\end{array}$ & $\begin{array}{l}\text { Eurostat und } \\
\text { nationales } \\
\text { statistisches } \\
\text { Amt }\end{array}$ & 1998 & $\begin{array}{l}\text { www.testh2.scb.se/ } \\
\text { tus/tus/ }\end{array}$ & Ein Jahr & $\begin{array}{l}\text { Personen ab } 15 \text { Jahre, die in } \\
\text { privaten Haushalten leben, ohne in } \\
\text { Gemeinschaftsunterkünften, } \\
\text { Heimen oder Anstalten lebende } \\
\text { Personen }\end{array}$ & $\begin{array}{l}12045 \text { Wohneinhei- } \\
\text { ten, von denen } \\
10330 \text { in der end- } \\
\text { gültigen Stichprobe } \\
\text { berücksichtigt } \\
\text { wurden, was } \\
\text { potenzielle } 16462 \text { in } \\
\text { Betracht kommenden } \\
\text { Personen entspricht }\end{array}$ & $\begin{array}{l}\text { Jeder Befragte füllt } \\
\text { Tagebücher für zwei } \\
\text { Tagebuchtage aus, die } \\
\text { jeweils } 24 \text { Stunden } \\
\text { abdecken }\end{array}$ & $\begin{array}{l}\text { Kurze, willkürliche Momente } \\
\text { im Leben der Menschen } \\
\text { werden beobachtet, die daher } \\
\text { nicht als repräsentativ } \\
\text { betrachtet werden können }\end{array}$ \\
\hline Italien & $\begin{array}{l}\text { Harmonised } \\
\text { European Time Use } \\
\text { Survey (HETUS) }\end{array}$ & $\begin{array}{l}\text { Eurostat und } \\
\text { nationales } \\
\text { statistisches } \\
\text { Amt }\end{array}$ & 2003 & $\begin{array}{l}\text { www.testh2.scb.se/ } \\
\text { tus/tus/ }\end{array}$ & Ein Jahr & $\begin{array}{l}\text { Alle in Italien ansässigen } \\
\text { Mitglieder privater Haushalte ab } \\
3 \text { Jahre, einschl. älterer Personen } \\
\text { (keine Altersobergrenze) }\end{array}$ & $\begin{array}{l}21075 \text { Haushalte mit } \\
55760 \text { Personen }\end{array}$ & $\begin{array}{l}\text { Jeder Befragte füllt } \\
\text { Tagebücher für zwei } \\
\text { Tagebuchtage aus, die } \\
\text { jeweils } 24 \text { Stunden } \\
\text { abdecken }\end{array}$ & $\begin{array}{l}\text { Kurze, willkürliche Momente } \\
\text { im Leben der Menschen } \\
\text { werden beobachtet, die daher } \\
\text { nicht als repräsentativ } \\
\text { betrachtet werden können }\end{array}$ \\
\hline Japan & $\begin{array}{l}\text { Survey on Time Use } \\
\text { and Leisure Activities }\end{array}$ & $\begin{array}{l}\text { Statistics } \\
\text { Bureau und } \\
\text { Statistical } \\
\text { Research } \\
\text { Training } \\
\text { Institute }\end{array}$ & 2006 & $\begin{array}{l}\text { www.stat.go.jp/ } \\
\text { English/data/shakai/ }\end{array}$ & $\begin{array}{l}\text { Zwei aufeinan- } \\
\text { derfolgende Tage } \\
\text { vom 14.-22. } \\
\text { Oktober } 2006\end{array}$ & $\begin{array}{l}\text { Alle Personen ab } 10 \text { Jahre, } \\
\text { einschl. in Japan lebender } \\
\text { Ausländer }\end{array}$ & $\begin{array}{l}80000 \text { Haushalte mit } \\
\text { rd. } 200000 \text { Personen }\end{array}$ & $\begin{array}{l}\text { Zwei Fragebogen: Bei } \\
\text { Fragebogen A wird eine } \\
\text { Vorkodierungsmethode } \\
\text { angewandt und in } \\
\text { Fragebogen B wird die } \\
\text { Zeitnutzung stärker im } \\
\text { Detail abgefragt }\end{array}$ & $\begin{array}{l}\text { Die Befragten erhalten in } \\
\text { Viertelstunden gegliederte } \\
\text { Tabellen zur Erfassung der } \\
\text { Zeitnutzung }\end{array}$ \\
\hline Kanada & $\begin{array}{l}\text { General Social } \\
\text { Survey } \\
\text { (Sondermodul) }\end{array}$ & $\begin{array}{l}\text { Statistics } \\
\text { Canada }\end{array}$ & 2005 & $\begin{array}{l}\text { http://cansim2. } \\
\text { statcan.ca/cgi-win/ } \\
\text { cnsmcgi.exe?Lang= } \\
\text { E\&RootDir=CII/ } \\
\text { \&ResultTemplate= } \\
\text { CII/CII_pick\& } \\
\text { Array_Pick= } \\
\text { 1\&Arrayld= } \\
1130001\end{array}$ & $\begin{array}{l}11 \text { gleich große } \\
\text { monatl. Stichproben } \\
\text { von Januar bis } \\
\text { November } \\
\text { (verlängert bis Mitte } \\
\text { Dezember) }\end{array}$ & $\begin{array}{l}\text { Nicht in Gemeinschafts- } \\
\text { unterkünften, Heimen oder } \\
\text { Anstalten lebende Personen ab } \\
15 \text { Jahre, die in den kanadischen } \\
\text { Provinzen leben, ohne Personen } \\
\text { ohne Telefon (2\% der } \\
\text { Bevölkerung) und Personen, die } \\
\text { lediglich ein Mobiltelefon besitzen } \\
\text { (rd. } 5 \% \text { ) }\end{array}$ & Rd. 25000 Personen & $\begin{array}{l}\text { Computergestützte } \\
\text { Telefonbefragung }\end{array}$ & $\begin{array}{l}\text { Teilstichproben füllen } \\
\text { besondere Module zu } \\
\text { „Teilnahme an Kultur-, Sport- } \\
\text { und Bewegungsaktivitäten“, } \\
\text { „Soziales Netzwerk und } \\
\text { Vertrauen“ und } \\
\text { „Verkehrsmittel“ aus }\end{array}$ \\
\hline Korea & Time Use Survey & $\begin{array}{l}\text { Korea National } \\
\text { Statistical Office }\end{array}$ & 2004 & $\begin{array}{l}\text { www.nso.go.kr/ } \\
\text { eng2006/ } \\
\text { e02_0000/ } \\
\text { e02c_0000/ } \\
\text { e02cb_0000/ } \\
\text { e02cb_0000.html }\end{array}$ & $\begin{array}{l}12 \text { Tage vom 2.-13. } \\
\text { September }\end{array}$ & Personen ab 10 Jahre & Rd. 12750 Haushalte & $\begin{array}{l}\text { Tagebuch für alle } \\
\text { Haushaltsmitglieder ab } \\
10 \text { Jahre (Erfassung der } \\
\text { Haupt- und gleichzeitig } \\
\text { erfolgenden Aktivitäten, } \\
\text { für die betreffenden } \\
\text { beiden Tage in 10- } \\
\text { Minuten-Intervalle } \\
\text { eingeteilt) }\end{array}$ & $\begin{array}{l}\text { Der Stichprobenrahmen wird } \\
\text { aus der Vielzweck- } \\
\text { Haushaltsstichprobe (HAF- } \\
\text { MP) erzeugt, die sich aus } \\
\text { dem Population and Housing } \\
\text { Census aus dem Jahr } 2000 \\
\text { ableitet }\end{array}$ \\
\hline
\end{tabular}




\begin{tabular}{|c|c|c|c|c|c|c|c|c|c|}
\hline & Name der Erhebung & Behörde & Jahr & $\begin{array}{l}\text { Website (Daten und } \\
\text { Dokumentation) }\end{array}$ & Erhebungszeitraum & Erfasste Bevölkerung & Stichprobenumfang & $\begin{array}{l}\text { Anzahl und Art } \\
\text { der Tagebuchtage }\end{array}$ & $\begin{array}{l}\text { Sonstige } \\
\text { Erhebungsmerkmale }\end{array}$ \\
\hline Mexiko & $\begin{array}{l}\text { Encuesta Nacional } \\
\text { sobre Uso del Tiempo } \\
\text { (ENUT) }\end{array}$ & $\begin{array}{l}\text { Instituto } \\
\text { Nacional de } \\
\text { Estadística, } \\
\text { Geografía e } \\
\text { Informática } \\
\text { (INEGI) }\end{array}$ & 2002 & $\begin{array}{l}\text { www.inegi.gob.mx/ } \\
\text { est/contenidos/ } \\
\text { espanol/proyectos/ } \\
\text { metadatos/ } \\
\text { encuestas/ } \\
\text { enut_2310.asp?s=est } \\
\& c=5440\end{array}$ & $\begin{array}{l}28 \text { Tage, verteilt auf } \\
4 \text { Runden im Umfang } \\
\text { von jeweils } 7 \text { Tagen }\end{array}$ & $\begin{array}{l}\text { Inländische Haushalte, die sich } \\
\text { regelmäßig in privaten } \\
\text { Wohnunterkünften im Staatsgebiet } \\
\text { aufhalten }\end{array}$ & $\begin{array}{l}5450 \text { tatsächlich } \\
\text { aufgesuchte und } \\
\text { interviewte Haushalte }\end{array}$ & & $\begin{array}{l}\text { ENUT ist ein Modul der } \\
\text { nationalen Erhebung zu den } \\
\text { Haushaltseinkommen und } \\
\text {-ausgaben (Encuesta Nacional } \\
\text { de Ingresos y Gastos de los } \\
\text { Hogares, ENIGH) }\end{array}$ \\
\hline Neuseeland & $\begin{array}{l}\text { Time Use Survey } \\
\text { (TUS) }\end{array}$ & $\begin{array}{l}\text { Statistics New } \\
\text { Zealand (SNZ) }\end{array}$ & $\begin{array}{l}1999 \\
\text { (ein- } \\
\text { malig) }\end{array}$ & $\begin{array}{l}\text { www2.stats.govt.nz/ } \\
\text { domino/external/ } \\
\text { omni/omni.nsf/ } \\
\text { outputs/ } \\
\text { Time+Use+Survey }\end{array}$ & $\begin{array}{l}\text { Zwischen Juli } 1998 \\
\text { und Juni } 1999\end{array}$ & $\begin{array}{l}\text { Alle nicht in Gemeinschafts- } \\
\text { unterkünften oder in Heimen bzw. } \\
\text { Anstalten lebenden Personen aus } \\
\text { der Zivilbevölkerung ab } 12 \text { Jahre, } \\
\text { die sich in privaten Haushalten } \\
\text { aufhalten }\end{array}$ & $\begin{array}{l}7200 \text { ausgewählte } \\
\text { Haushalte mit einem } \\
\text { erwart. Stichproben- } \\
\text { umfang von insg. rd. } \\
8500 \text { Personen }\end{array}$ & $\begin{array}{l}\text { Im Mittelpunkt der } \\
\text { Daten stehen die vier } \\
\text { Grundkategorien der } \\
\text { Zeit (verpflichtete Zeit, } \\
\text { verplante Zeit, nötige } \\
\text { Zeit, freie Zeit) }\end{array}$ & \\
\hline Norwegen & $\begin{array}{l}\text { Harmonised } \\
\text { European Time Use } \\
\text { Survey (HETUS) }\end{array}$ & $\begin{array}{l}\text { Eurostat und } \\
\text { nationales } \\
\text { statistisches } \\
\text { Amt }\end{array}$ & 2001 & $\begin{array}{l}\text { www.testh2.scb.se/ } \\
\text { tus/tus/ }\end{array}$ & Ein Jahr & $\begin{array}{l}\text { Alle Personen im Alter von 9- } \\
79 \text { Jahren (mit einer zusätzl. } \\
\text { Stichprobe der 60- bis 66- } \\
\text { Jährigen), die in Norwegen } \\
\text { gemeldet sind }\end{array}$ & $\begin{array}{l}\text { Hauptstichprobe von } \\
6470 \text { Personen }\end{array}$ & $\begin{array}{l}\text { Jeder Befragte füllt } \\
\text { Tagebücher für zwei } \\
\text { Tagebuchtage aus, die } \\
\text { jeweils } 24 \text { Stunden } \\
\text { abdecken }\end{array}$ & $\begin{array}{l}\text { Kurze, willkürliche Momente } \\
\text { im Leben der Menschen } \\
\text { werden beobachtet, die daher } \\
\text { nicht als repräsentativ } \\
\text { betrachtet werden können }\end{array}$ \\
\hline Polen & $\begin{array}{l}\text { Harmonised } \\
\text { European Time Use } \\
\text { Survey (HETUS) }\end{array}$ & $\begin{array}{l}\text { Eurostat und } \\
\text { nationales } \\
\text { statistisches } \\
\text { Amt }\end{array}$ & $\begin{array}{l}2004 \\
\text { (ein- } \\
\text { malig) }\end{array}$ & $\begin{array}{l}\text { www.testh2.scb.se/ } \\
\text { tus/tus/ }\end{array}$ & Ein Jahr & $\begin{array}{l}\text { Personen ab } 15 \text { Jahre, wobei die } \\
\text { Mitglieder der ausgewählten } \\
\text { Haushalte sechs sozioökono- } \\
\text { mische Gruppen repräsentieren }\end{array}$ & $\begin{array}{l}10256 \text { ausgewählte } \\
\text { Haushalte }\end{array}$ & $\begin{array}{l}\text { Jeder Befragte füllt } \\
\text { Tagebücher für zwei } \\
\text { Tagebuchtage aus, die } \\
\text { jeweils } 24 \text { Stunden } \\
\text { abdecken }\end{array}$ & $\begin{array}{l}\text { Ein Werktag und ein } \\
\text { Wochenendtag (Samstag } \\
\text { oder Sonntag) vor oder nach } \\
\text { dem betreffenden Werktag } \\
\text { werden den einzelnen } \\
\text { Wohneinheiten der } \\
\text { Hauptstichprobe nach dem } \\
\text { Zufallsprinzip zugewiesen }\end{array}$ \\
\hline Schweden & $\begin{array}{l}\text { Harmonised } \\
\text { European Time Use } \\
\text { Survey (HETUS) }\end{array}$ & $\begin{array}{l}\text { Eurostat und } \\
\text { nationales } \\
\text { statistisches } \\
\text { Amt }\end{array}$ & 2001 & $\begin{array}{l}\text { www.testh2.scb.se/ } \\
\text { tus/tus/ }\end{array}$ & Ein Jahr & $\begin{array}{l}\text { Personen im Alter von 20- } \\
84 \text { Jahre, die im Erhebungs- } \\
\text { zeitraum in Schweden gemeldet } \\
\text { sind }\end{array}$ & $\begin{array}{l}2138 \text { Haushalte mit } \\
3980 \text { Personen }\end{array}$ & $\begin{array}{l}\text { Jeder Befragte füllt } \\
\text { Tagebücher für zwei } \\
\text { Tagebuchtage aus, die } \\
\text { jeweils } 24 \text { Stunden } \\
\text { abdecken }\end{array}$ & $\begin{array}{l}\text { Die Haushaltsstichprobe wird } \\
\text { erzeugt, indem eine Stich- } \\
\text { probe von Einzelpersonen } \\
\text { gezogen und diesen } \\
\text { ausgewählten Personen ein } \\
\text { Partner hinzugefügt wird, } \\
\text { was bedeutet, dass wir nicht } \\
\text { wissen, wie viele Personen } \\
\text { die Haushaltsstichprobe } \\
\text { umfasste, sondern lediglich, } \\
\text { wie viele Personen sich an } \\
\text { der Erhebung beteiligt haben }\end{array}$ \\
\hline
\end{tabular}


Table 2.A1.1. Dokumentation der Methodiken nationaler Erhebungen zur Zeitnutzung (Forts.)

\begin{tabular}{|c|c|c|c|c|c|c|c|c|c|}
\hline & Name der Erhebung & Behörde & Jahr & $\begin{array}{l}\text { Website (Daten und } \\
\text { Dokumentation) }\end{array}$ & Erhebungszeitraum & Erfasste Bevölkerung & Stichprobenumfang & $\begin{array}{l}\text { Anzahl und Art } \\
\text { der Tagebuchtage }\end{array}$ & $\begin{array}{l}\text { Sonstige } \\
\text { Erhebungsmerkmale }\end{array}$ \\
\hline Spanien & $\begin{array}{l}\text { Harmonised } \\
\text { European Time Use } \\
\text { Survey (HETUS) }\end{array}$ & $\begin{array}{l}\text { Eurostat und } \\
\text { nationales } \\
\text { statistisches } \\
\text { Amt }\end{array}$ & 2003 & $\begin{array}{l}\text { www.testh2.scb.se/ } \\
\text { tus/tus/ }\end{array}$ & Ein Jahr & $\begin{array}{l}\text { Alle Mitglieder regulärer privater } \\
\text { Haushalte von Gebietsansässigen } \\
\text { ab } 10 \text { Jahre }\end{array}$ & $\begin{array}{l}20603 \text { Haushalte mit } \\
46774 \text { Personen }\end{array}$ & $\begin{array}{l}\text { Jeder Befragte füllt } \\
\text { Tagebücher für zwei } \\
\text { Tagebuchtage aus, die } \\
\text { jeweils } 24 \text { Stunden } \\
\text { abdecken }\end{array}$ & $\begin{array}{l}\text { Alle Tage des Jahres werden } \\
\text { erfasst }\end{array}$ \\
\hline Türkei & Time Use Survey & $\begin{array}{l}\text { Turkish } \\
\text { Statistical } \\
\text { Institute } \\
\text { (Turkstat) }\end{array}$ & 2006 & $\begin{array}{l}\text { www.turkstat.gov.tr/ } \\
\text { PreHaberBultenleri.d } \\
\text { o?id=528 }\end{array}$ & Ein Jahr & Haushaltsmitglieder ab 15 Jahre & $\begin{array}{l}5070 \text { ausgewählte } \\
\text { Haushalte, aus denen } \\
11815 \text { Haushalts- } \\
\text { mitglieder ab } \\
15 \text { Jahre interviewt } \\
\text { werden }\end{array}$ & $\begin{array}{l}\text { Zwei Tagebücher: eines } \\
\text { für einen Werktag und } \\
\text { eines für einen Wochen- } \\
\text { endtag, die täglichen } \\
\text { Aktivitäten werden über } \\
24 \text { Stunden in Zehn- } \\
\text { Minuten-Intervallen } \\
\text { erfasst }\end{array}$ & \\
\hline $\begin{array}{l}\text { Vereinigtes } \\
\text { Königreich }\end{array}$ & $\begin{array}{l}\text { Harmonised } \\
\text { European Time Use } \\
\text { Survey (HETUS) }\end{array}$ & $\begin{array}{l}\text { Eurostat und } \\
\text { nationales } \\
\text { statistisches } \\
\text { Amt }\end{array}$ & 2001 & $\begin{array}{l}\text { www.testh2.scb.se/ } \\
\text { tus/tus/ }\end{array}$ & Juni 2000 - Juli 2001 & $\begin{array}{l}\text { Alle Mitglieder eines ausgewählten } \\
\text { Haushalts ab } 8 \text { Jahre (obgleich in } \\
\text { der Datenbank letztlich lediglich } \\
\text { die über 10-Jährigen } \\
\text { berücksichtigt sind) }\end{array}$ & $\begin{array}{l}11854 \text { in die Stich- } \\
\text { probe einbezogene } \\
\text { Haushalte, woraus } \\
\text { sich } 20991 \text { Tage- } \\
\text { bücher ergeben }\end{array}$ & $\begin{array}{l}\text { Jeder Befragte füllt } \\
\text { Tagebücher für zwei } \\
\text { Tagebuchtage aus, die } \\
\text { jeweils } 24 \text { Stunden } \\
\text { abdecken }\end{array}$ & $\begin{array}{l}\text { Kurze, willkürliche Momente } \\
\text { im Leben der Menschen } \\
\text { werden beobachtet, die daher } \\
\text { nicht als repräsentativ } \\
\text { betrachtet werden können }\end{array}$ \\
\hline $\begin{array}{l}\text { Vereinigte } \\
\text { Staaten }\end{array}$ & $\begin{array}{l}\text { American Time Use } \\
\text { Survey (ATUS) }\end{array}$ & $\begin{array}{l}\text { Bureau of Labor } \\
\text { Statistics (BLS) }\end{array}$ & 2005 & www.bls.gov/tus/ & Volles Kalenderjahr & $\begin{array}{l}\text { Personen ab } 15 \text { Jahre, die in } \\
\text { privaten Haushalten leben }\end{array}$ & Rd. 13000 Personen & $\begin{array}{l}\text { Den ausgewählten } \\
\text { Personen wird ein } \\
\text { Wochentag zugewiesen, } \\
\text { an dem die Aktivitäten } \\
\text { erfasst werden. 25\% } \\
\text { der Stichprobe wird ein } \\
\text { Wochenendtag } \\
\text { zugewiesen }\end{array}$ & $\begin{array}{l}\text { Die zu erfassenden Tage } \\
\text { werden zugewiesen, um } \\
\text { Verzerrungen auszu- } \\
\text { schließen, die sich u.U. } \\
\text { ergeben würden, wenn die } \\
\text { Befragten die Zeit nach } \\
\text { eigenem Gutdünken erfassen } \\
\text { würden }\end{array}$ \\
\hline Multinational & $\begin{array}{l}\text { Multinational Time } \\
\text { Use Survey (MTUS) }\end{array}$ & $\begin{array}{l}\text { Centre for Time } \\
\text { Use Research }\end{array}$ & 2006 & $\begin{array}{l}\text { www.timeuse.org/ } \\
\text { mtus/ }\end{array}$ & Ein Jahr & $\begin{array}{l}\text { Die Bevölkerung im Alter von } \\
\text { 20-59 Jahren }\end{array}$ & & $\begin{array}{l}\text { Jeder Eintrag erfolgt auf } \\
\text { der Grundlage von 7- } \\
\text { Tages-Tagebüchern, für } \\
\text { die Durchschnittswerte } \\
\text { ermittelt werden }\end{array}$ & $\begin{array}{l}\text { Der MTUS-Datensatz } \\
\text { umfasst rd. } 20 \text { Länder und } \\
\text { wird regelmäßig erweitert }\end{array}$ \\
\hline
\end{tabular}



Kapitel 3

\section{Interpretation der OECD-Indikatoren}




\section{Der Zweck von Sozialindikatoren}

Gesellschaft auf einen Blick 2009 trägt zur Beantwortung von zwei Fragen bei:

- Welche Fortschritte haben die OECD-Länder gegenüber ihrer eigenen Vergangenheit und im Vergleich zu anderen Ländern im Hinblick auf ihre soziale Entwicklung erreicht?

- Wie wirksam waren die Maßnahmen der Gesellschaft, um die soziale Entwicklung voranzubringen?

Zur Beantwortung der ersten Frage bedarf es Indikatoren, die länderübergreifend und im Zeitverlauf ein breites Spektrum von Ergebnissen im Sozialbereich abdecken. Da die soziale Entwicklung Verbesserungen in den Bereichen Gesundheit, Bildung und Wirtschaftsressourcen sowie eine stabile Grundlage für soziale Interaktionen voraussetzt, müssen für all diese Dimensionen Indikatoren gefunden werden.

Die zweite Frage zur Wirksamkeit gesellschaftlicher Maßnahmen lässt sich sogar noch schwerer beantworten. Gesellschaften versuchen, die Ergebnisse im Sozialbereich - häufig über die staatliche Politik - zu beeinflussen. Eine entscheidende Frage ist, ob diese staatlichen Maßnahmen wirksam sind, um die Ziele zu erreichen. Indikatoren helfen diese Frage zu prüfen. Ein erster Schritt besteht darin, die Ressourcen zu vergleichen, mit denen in den einzelnen Ländern versucht wird, eine Veränderung der Ergebnisse zu bewirken, und diese Ressourcen den Ergebnissen im Sozialbereich gegenüberzustellen. Auch wenn ein derartiger Vergleich weit von einer umfassenden Evaluierung der Wirksamkeit der Maßnahmen entfernt ist, können die Indikatoren dennoch dazu beitragen, jene Bereiche hervorzuheben, in denen mehr Analysearbeit erforderlich sein könnte.

\section{Der Rahmen der OECD-Sozialindikatoren}

Auch wenn die hier gewählte Struktur keinen umfassenden Rahmen für Sozialindikatoren bietet, handelt es sich dennoch um mehr als um eine einfache Auflistung von Indikatoren. Dieser Rahmen orientiert sich an den in anderen OECDTätigkeitsfeldern gesammelten Erfahrungen und Informationen im Hinblick auf die Beurteilung der Politikmaßnahmen und Ergebnisse in einer Vielzahl von Bereichen. Dieser Rahmen macht sich insbesondere die von der OECD gesammelten Erfahrungen mit Umweltindikatoren zu Nutze. Diese Indikatoren sind in einen als PSR-Modell (PressureState-Response) bekannten Rahmen integriert*. Nach diesem Modell üben menschliche Aktivitäten Druck (pressure) auf die Umwelt aus, der seinerseits die Naturressourcen und den Umweltzustand (state) beeinflusst und die Gesellschaft dazu bewegt, auf diese Veränderungen mittels verschiedener Maßnahmen zu reagieren (societal response). Der PSRRahmen hebt diese sequenziellen Verknüpfungen hervor, was wiederum den

\footnotetext{
* Der PSR-Rahmen ist wiederum eine Variante eines Konzepts, das dem von der VN-Kommission für nachhaltige Entwicklung verwendeten „Driving force-State-Response“-Modell (DSR) und dem von der Europäischen Umweltbehörde verwendeten „Driving force-Pressure-State-Impact-Response“-Modell (DPSIR) zu Grunde liegt.
} 
Entscheidungsträgern und der Öffentlichkeit hilft, die oft übersehenen Zusammenhänge zwischen Umwelt- und sonstigen Problemen zu erkennen.

Ein ähnliches Konzept findet im vorliegenden Bericht auf die Sozialindikatoren Anwendung. Die Indikatoren werden anhand von zwei Dimensionen klassifiziert, von denen die erste die Natur dieser Indikatoren betrachtet und sie wiederum in drei Bereiche unterteilt:

- Die Indikatoren für den sozialen Kontext beziehen sich auf Variablen, die, ohne unmittelbare Politikziele zu sein, für das Verständnis des sozialpolitischen Kontextes entscheidend sind. So ist beispielsweise der Anteil älterer Menschen an der Gesamtbevölkerung kein Politikziel. Er liefert aber wichtige Informationen über das soziale Umfeld, in dem z.B. gesundheits-, steuer- oder rentenpolitische Maßnahmen getroffen werden. Im Unterschied $z u$ anderen Indikatoren können Trends der Indikatoren für den sozialen Kontext nicht eindeutig als "gut" oder "schlecht" interpretiert werden.

- Die Indikatoren für den sozialen Status beschreiben die Ergebnisse im Sozialbereich, die Politikmaßnahmen zu beeinflussen suchen. Diese Indikatoren beschreiben die allgemeinen Bedingungen für die Bevölkerung. Im Idealfall werden Indikatoren gewählt, die einfach und eindeutig interpretiert werden können - so hätten beispielsweise alle Länder lieber niedrige als hohe Armutsquoten.

- Die Indikatoren für die Reaktion der Gesellschaft liefern Informationen über die konkreten Maßnahmen, die die Gesellschaft zur Beeinflussung des sozialen Status ergreift. Hierzu gehören Indikatoren für die Weichenstellung der staatlichen Politik. Aber auch zusätzliche Aktivitäten von Nichtregierungsorganisationen (NRO), Familien und der Zivilgesellschaft im weiteren Sinne zählen zur Reaktion der Gesellschaft. Eine Gegenüberstellung der Indikatoren für die Reaktion der Gesellschaft und den Indikatoren für den sozialen Status kann erste Aufschlüsse über die Wirksamkeit der staatlichen Politik geben.

Bei der Einordnung der Sozialindikatoren in eine der drei oben genannten Gruppen ist die Wahl zwischen Kontext und Status nicht immer eindeutig. Die Geburtenziffern können beispielsweise in Ländern wie Frankreich das Ziel geburtenfreundlicher Maßnahmen sein, in anderen Ländern wie im Vereinigten Königreich dagegen ein Indikator für den sozialpolitischen Kontext. Entsprechend kann Familienzerfall in einigen Ländern als Ergebnis einer gescheiterten öffentlichen Politik betrachtet werden, während er in anderen Ländern gar kein ausdrückliches Politikthema ist.

Ein die Aussagekraft der hier verwendeten Indikatoren für den sozialen Kontext, den sozialen Status und die Reaktion der Gesellschaft stark einschränkender Faktor ist die Tatsache, dass sich die Indikatoren in diesem Bericht auf die nationale Ebene beziehen. In Mitgliedsländern mit in hohem Maße föderalistisch geprägter Verwaltungsstruktur, wie z.B. den Vereinigten Staaten, Kanada und Australien spiegeln die Indikatoren möglicherweise nicht die einzelnen Regionen wider, die einen anderen sozialen Kontext, unterschiedliche Ergebnisse und Reaktionen der Gesellschaft aufweisen können. Dies sollte bei der Untersuchung der hier vorgestellten Indikatoren berücksichtigt werden.

Die zweite Dimension des OECD-Rahmens gruppiert die Indikatoren anhand der von ihnen abgedeckten globalen Politikfelder. Die Klassifizierung der Indikatoren des sozialen 
Status und der Reaktionen der Gesellschaft erfolgt nach vier allgemeinen Zielen der Sozialpolitik:

- Autonomie ist ein grundlegendes Ziel der Sozialpolitik, das auch im Kommuniqué der OECD-Tagung der Sozial- und Gesundheitsminister (www.oecd.org/socmin2005) ausdrücklich formuliert wurde. Die Autonomie des Einzelnen wird erhöht, indem seine aktive Teilnahme am Wirtschafts- und Gesellschaftsleben wie auch die Selbstständigkeit in Aktivitäten des täglichen Lebens gefördert wird.

- Soziale Gerechtigkeit ist ein weiteres seit langem verfolgtes Ziel der Sozialpolitik. Die Ergebnisse im Hinblick auf die soziale Gerechtigkeit werden vor allem anhand des Zugangs von Einzelpersonen und Familien zu Ressourcen gemessen.

- Die Gesundheit ist zwar ein fundamentales Anliegen der Gesundheitsversorgungssysteme, doch ist für eine Verbesserung des Gesundheitszustands auch ein breiterer, die sozialen Bestimmungsfaktoren mit einbeziehender Fokus erforderlich, was die Gesundheit zu einem zentralen Ziel der Sozialpolitik macht.

- Der soziale Zusammenhalt wird häufig als übergeordnetes Ziel der Sozialpolitik eines Landes identifiziert. Während wenig Übereinstimmung hinsichtlich der genauen Bedeutung dieses Begriffs besteht, gibt es dennoch eine Reihe von Anhaltspunkten, die Aufschluss über einen Mangel an sozialem Zusammenhalt liefern. Eher positiv wird der soziale Zusammenhalt durch den Grad erkennbar, in dem sich der Einzelne am Leben der Gemeinschaft beteiligt, in der er lebt.

Der den OECD-Sozialindikatoren zu Grunde liegende Rahmen lässt sich als eine summarische „Matrix“ darstellen (Tabelle 3.1). Tabelle 3.1 liefert zusätzliche Informationen über die in den vorigen Ausgaben von Gesellschaft auf einen Blick enthaltenen Sozialindikatoren sowie die Indikatoren der vorliegenden Ausgabe.

\section{Auswahl und Beschreibung der Indikatoren}

Die Erfassung und Veröffentlichung der Sozialindikatoren weicht in den OECDLändern erheblich voneinander ab. Bei der Auswahl der Indikatoren für diesen Bericht wurden folgende Punkte berücksichtigt:

- Welches ist das Mindestmaß an Vergleichbarkeit der Indikatoren zwischen den Ländern? Dieser Bericht ist bestrebt, für jeden untersuchten Bereich die am besten vergleichbaren Informationen zu liefern. Die hier dargelegten Indikatoren beschränken sich jedoch nicht auf jene mit „absoluter“ Vergleichbarkeit. Der Leser wird aber auf die Natur der verwendeten Daten und die jeweils mit ihnen verbundene Problematik aufmerksam gemacht.

- Welches ist die Mindestanzahl von Ländern, für die die entsprechenden Daten verfügbar sein müssen? Generell gilt, dass diese Ausgabe nur jene Indikatoren enthält, die in der Mehrzahl der OECD-Länder verfügbar sind.

- Welche Datenaufschlüsselung sollte auf Länderebene verwendet werden? Sozialindikatoren können auf Länderebene häufig in Ergebnisse nach Unterkategorien aufgeteilt werden, wie beispielsweise Alter, Geschlecht und Familienstand von Personen. Aus pragmatischen Gründen ist die hier vorgenommene Aufschlüsselung je nach dem betrachteten Indikator unterschiedlich.

Für jeden ausgewählten Indikator enthalten die Kapitel 4 bis 8 dieses Berichts eine Beschreibung der Hauptmerkmale und allgemeine Informationen zu Definition und 
Tabelle 3.1. In den fünf Ausgaben von Gesellschaft auf einen Blick (2001, 2003, 2005, 2006, 2009) erfasste Sozialindikatoren

\begin{tabular}{|c|c|c|c|c|c|c|}
\hline & & \multicolumn{5}{|c|}{ Inhalt } \\
\hline & & & Autonomie (SS) & Soziale Gerechtigkeit (EQ) & Gesundheit (HE) & Sozialer Zusammenhalt (CO) \\
\hline \multirow{3}{*}{ 衰 } & 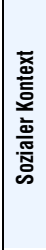 & $\begin{array}{l}\text { - Nationaleinkommen } \\
\text { - Migration } \\
\text { - Geburtenziffern (2001, 2005, 2006, 2009) } \\
\text { - Eheschließungen und Scheidungen (2001, } \\
\text { 2005, 2006, 2009) } \\
\text { - Abhängigenquotienten (2001, 2003, 2005, 2006) } \\
\text { - Flüchtlinge und Asylbewerber (2001) } \\
\text { - Alleinerziehende Eltern (2001) }\end{array}$ & & & & \\
\hline & 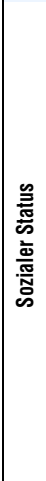 & & $\begin{array}{l}\text { - Beschäftigung } \\
\text { - Arbeitslosigkeit } \\
\text { - Erwerbstätige Mütter }(2001,2003,2005,2006) \\
\text { - Erwerbslosenhaushalte }(2001,2003,2005,2006) \\
\text { - Schülerleistungen (2001, 2003, 2005, 2009) } \\
\text { - Nicht in Beschäftigung, Bildung oder } \\
\text { Ausbildung (2001, 2005, 2009) } \\
\text { - Alter des Ausscheidens aus dem Erwerbsleben } \\
\text { (2001, 2005, 2009) } \\
\text { - Kinderbetreuungskosten (2006) } \\
\text { - Kinderbetreuung (2001, 2009) }\end{array}$ & $\begin{array}{l}\text { - Einkommensungleichheit (2001, 2005, 2009) } \\
\text { - Armut (2001, 2005, 2009) } \\
\text { - Kinderarmut (2005, 2009) } \\
\text { - Einkommen älterer Personen (2003, 2005) } \\
\text { - Niedriglohnbeschäftigung (2001) } \\
\text { - Lohngefällle zwischen Männern und Frauen } \\
\text { (2001, 2006) } \\
\text { - Materielle Entbehrung (2006) } \\
\text { - Armutspersistenz (2006) } \\
\text { - Generationenmobilität (2006) } \\
\text { - Wohnkosten (2006) }\end{array}$ & $\begin{array}{l}\text { - Lebenserwartung (2001, 2005, 2006, 2009) } \\
\text { - Lebenserwartung nach Gesundheitszustand } \\
\text { (2003, 2005) } \\
\text { - Selbsteinschätzung des Gesundheitszustands (2009) } \\
\text { - Säuglingssterblichkeit (2001, 2005, 2009) } \\
\text { - Niedriges Geburtsgewicht (2003, 2006, 2009) } \\
\text { - Fettleibigkeit (2003, 2009) } \\
\text { - Körpergröße (2009) } \\
\text { - Seelische Gesundheit (2009) } \\
\text { - Potenziell verlorene Lebensjahre (2001, 2003) } \\
\text { - Behinderungsfreie Lebenserwartung (2001) } \\
\text { - Unfälle (2001) } \\
\text { - Krankheitsbedingte Abwesenheit vom Arbeitsplatz } \\
\text { (2006) } \\
\text { - Gesundheitl. Ungleichheiten (2006) }\end{array}$ & $\begin{array}{l}\text { - Suizide } \\
\text { - Lebenszufriedenheit (2005, 2006, 2009) } \\
\text { - Kriminalitätsbedingte Viktimisierung (2001, 2003, 2009) } \\
\text { - Arbeitszufriedenheit (2009) } \\
\text { - Aggressives Verhalten (Bullying) in der Schule (2009) } \\
\text { - Risikoverhalten (2009) } \\
\text { - Soziale Isolierung (2001, 2005) } \\
\text { - Mitgliedschaft in Vereinen (2001, 2005) } \\
\text { - Geburten im Teenageralter (2003, 2005) } \\
\text { - Suchtverhalten und damit zusammen- hängende } \\
\text { Todesfälle (2001, 2005) } \\
\text { - Streiks und Aussperrungen (2001, 2003, 2006) } \\
\text { - Wahlbeteiligung (2001, 2006) } \\
\text { - Jugendkriminalität (2003) } \\
\text { - Vertrauen in den öffentlichen Dienst (2006) } \\
\text { - Arbeitsunfällle (2001, 2006) }\end{array}$ \\
\hline & 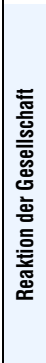 & & $\begin{array}{l}\text { - Angemessenheit der } \\
\text { Mindestsicherungsleistungen } \\
\text { - Aktivierungsmaßnahmen (2001) } \\
\text { - Bildungsausgaben (2001, 2009) } \\
\text { - Frühkindliche Bildung und Erziehung (2001) } \\
\text { - Lesekompetenz Erwachsener (2001) } \\
\text { - Steuer- und Abgabenkeil auf den Faktor Arbeit } \\
\text { (2001, 2006) } \\
\text { - Schüler mit Benachteiligungen (2003) } \\
\text { - Ressourcen behinderter Erwachsener (2002) } \\
\text { - Erwerbstätige mit Behinderungen (2003) } \\
\text { - Mindestsicherungsleistungen (2005) }\end{array}$ & $\begin{array}{l}\text { - Öffentliche Sozialausgaben } \\
\text { - Gesamtsozialausgaben } \\
\text { - Private Sozialausgaben }(2001,2005) \\
\text { - Leistungsempfänger }(2001,2005) \\
\text { - Verdienstungleichheiten }(2006) \\
\text { - Mindestlöhne (2001) } \\
\text { - Ersatzquoten der Altersrenten }(2005,2006) \\
\text { - Rentenversprechen (2005) }\end{array}$ & $\begin{array}{l}\text { - Gesundheitsausgaben } \\
\text { - Verantwortung für die Finanzierung der } \\
\text { Gesundheitsversorgung }(2001,2003) \\
\text { - Langzeitpflegebedürttige }(2001,2005,2006,2009) \\
\text { - Gesundheitsinfrastruktur }(2001)\end{array}$ & • Gefängnisbevö|kerung $(2001,2003)$ \\
\hline
\end{tabular}

Anmerkung: Die Daten beziehen sich auf die in Gesellschaft auf einen Blick erfassten „Bereiche“. Die kursiv gedruckten Indikatoren sind in allen fünf Ausgaben von Gesellschaft auf einen Blick erschienen. Die Bezeichnungen für die einzelnen Indikatorkategorien sind der Ausgabe 2009 entnommen; zugleich sind einige Indikatoren von einer Kategorie in eine andere verlagert worden. 
Messung. Die meisten Indikatoren existieren bereits in der einen oder anderen Form. Einige werden auf regelmäßiger Basis in anderen OECD-Veröffentlichungen publiziert (z.B. Labour Force Statistics, Social Expenditure Database und OECD-Gesundheitsdaten); andere wurden auf Ad-hoc-Basis zusammengetragen.

Einzelne Indikatoren können für mehrere Bereiche der Sozialpolitik von Bedeutung sein, d.h. sie können mehr als einer Kategorie zugeordnet werden. So ist z.B. die Fähigkeit, Aktivitäten des täglichen Lebens ohne Hilfe durchzuführen, potenziell ein Indikator für sozialen Zusammenhalt, Autonomie und Gesundheit. Die Indikatoren sind hier in der Kategorie aufgeführt, für die sie offenbar die größte Rolle spielen.

Im gesamten Bericht dient der in Klammern stehende Code hinter jedem einzelnen Indikator (z.B. GE1) der Zuordnung desselben zu einem Politikfeld (wie in den unten stehenden Tabellen aufgeführt), und die Nummerierung der Indikatoren dient der Vereinfachung von Querverweisen. Obwohl Bezeichnung und Code der in dieser Ausgabe erfassten Indikatoren von denen in früheren Ausgaben von Gesellschaft auf einen Blick abweichen können, werden Anstrengungen unternommen, um in den untersuchten Politikfeldern Kontinuität zu gewährleisten.

\section{Indikatoren für den allgemeinen sozialen Kontext (GE)}

Beim Vergleich der Indikatoren für den sozialen Status und die Reaktion der Gesellschaft kann leicht der Schluss gezogen werden, dass ein Land im Vergleich zu anderen schlecht abschneidet oder dass wieder ein anderes Land im Vergleich zu anderen sehr viel Geld für ein spezifisches Politikziel ausgibt. Wichtig ist es, derartige Aussagen in einen globaleren Kontext einzuordnen. So ist beispielsweise das Nationaleinkommen in den OECD-Ländern unterschiedlich. Besteht also ein Zusammenhang zwischen Einkommen und Gesundheit, könnten die gesundheitlichen Bedingungen in reicheren Ländern unabhängig von der Reaktion der Gesellschaft besser sein als in ärmeren. Falls die Nachfrage nach Gesundheitsleistungen mit dem Einkommen steigt (was der Fall zu sein scheint), könnten reichere Länder (in Prozent des Nationaleinkommens) mehr Geld für die Gesundheitsversorgung aufwenden als ärmere. Das bedeutet aber nicht, dass die Indikatoren für den Gesundheitszustand und die Gesundheitsausgaben irreführend sind. Es bedeutet nur, dass bei Überlegungen über die Politikimplikationen der den Daten zu Grunde liegende allgemeine Kontext berücksichtigt werden sollte.

Sozialkontextindikatoren sind für die Interpretation vieler in dieser Veröffentlichung enthaltener Indikatoren von Bedeutung. Hierzu zählen das Pro-Kopf-Nationaleinkommen (GE1), das Auswirkung auf Qualität, Quantität und Natur der Leistungen in den Bereichen soziale Sicherung und Bildung hat, die eine Gesellschaft bieten kann, aber auch Geburtenziffern (GE2), Migration (GE3) sowie Eheschließungen und Scheidungen (GE4).

Liste der allgemeinen Kontextindikatoren (GE)

\begin{tabular}{ll}
\hline GE1. & Pro-Kopf-Nationaleinkommen \\
GE2. & Geburtenziffern \\
GE3. & Migration \\
GE4. & Eheschließungen und Scheidungen \\
\hline
\end{tabular}




\section{Autonomie (SS)}

Eine bezahlte Beschäftigung (SS1) ist für einen Großteil der Bevölkerung im erwerbsfähigen Alter ein wichtiger Faktor, um Geld, eine Identität, gesellschaftliche Interaktionen und einen sozialen Status zu erwerben. Darüber hinaus werden alle sozialen Sicherungssysteme durch die Beitragszahlungen der Erwerbstätigen finanziert. So ist auch die Förderung einer höher entlohnten Beschäftigung für alle OECD-Länder eine Priorität. Bei Arbeitslosigkeit (SS2) ist es dem Betreffenden, obwohl er dem Arbeitsmarkt zur Verfügung steht, nicht immer möglich, sich und seine Familie durch eine Erwerbstätigkeit $\mathrm{zu}$ versorgen. Die frühen Grundlagen spielen in Bezug auf die kognitive und soziale Entwicklung von Kindern eine wichtige Rolle, was wiederum im Hinblick auf die künftige Autonomie des Einzelnen von Bedeutung ist. Die Kinderbetreuungsrate (SS3) liefert Informationen über den Grad, in dem Kinder Zugang zu Einrichtungen frühkindlichen Lernens haben. Schülerleistungen (SS4) sind ein Indikator für eine wichtige Dimension der Humankapitalakkumulation am anderen Ende des Kindheitszyklus. Gute Schülerleistungen ermöglichen in der Zukunft längerfristige Autonomie, u.a. in bezahlter Beschäftigung. Weil Nachteile im frühen Erwachsenenalter häufig ein Indikator für langfristige Arbeitsmarktnachteile sind, kann eine Messgröße der Inaktivität von Jugendlichen Informationen über die Chancen für einen erfolgreichen Übergang in ein autonomes Erwerbsleben liefern (SS5).

Die Reaktionen der Gesellschaft auf Schülerleistungen und Inaktivität der Jugendlichen galten häufig der Gestaltung der Strukturen und Anreize in Schulsystemen (was schwer durch einen Sozialindikator zusammenzufassen ist), kamen aber auch in hohen öffentlichen und privaten Ausgaben für Bildung (SS7) zum Ausdruck.

Die nachstehende Tabelle enthält eine Auflistung der Indikatoren des sozialen Status und der Reaktion der Gesellschaft, die für die Beurteilung der Frage, ob OECD-Länder die Ziele zur Gewährleistung der Autonomie von Einzelnen und ihren Familien erreicht haben, besonders relevant sind.

Liste der Autonomie-Indikatoren (SS)

\begin{tabular}{llll}
\hline \multicolumn{2}{l}{ Sozialer Status } & \multicolumn{2}{l}{ Reaktion der Gesellschaft } \\
\hline SS1. & Beschäftigung & SS5. & Nicht in Beschäftigung, Bildung oder Ausbildung \\
SS2. & Arbeitslosigkeit & SS6. & Alter bei Austritt aus dem Erwerbsleben \\
SS3. & Kinderbetreuung & SS7. & Bildungsausgaben \\
SS4. & Schülerleistungen & & \\
EQ1. & Einkommensungleichheit & EQ4. & Angemessenheit von Mindestsicherungsleistungen \\
EQ2. & Armut & EQ5. & Öffentliche Sozialausgaben \\
EQ3. & Kinderarmut & EQ6. & Gesamtsozialausgaben \\
\hline
\end{tabular}

Anmerkung: Kursiv gedruckt sind jene Indikatoren, die trotz ihres Erscheinens in einer anderen Unterkategorie auch für die Beurteilung der Autonomie relevant sind.

\section{Soziale Gerechtigkeit (EQ)}

Dieser Begriff hat zahlreiche Dimensionen und kann sich u.a. auf die Zugangsmöglichkeiten zu sozialen Diensten, aber auch auf wirtschaftliche Chancen bzw. Gerechtigkeit im Hinblick auf die tatsächlichen Ergebnisse beziehen. Die Auffassungen darüber, was unter einer gerechten Verteilung der Ergebnisse oder Chancen genau zu verstehen ist, gehen weit auseinander. Da es zudem schwierig ist, Informationen über alle 
Dimensionen der sozialen Gerechtigkeit zu erhalten, beschränken sich die Indikatoren des sozialen Status auf Ungleichheiten bei den finanziellen Ressourcen.

Die Einkommensungleichheit (EQ1) ist ein natürlicher Ausgangspunkt für die Beurteilung der sozialen Gerechtigkeit der Gesellschaft insgesamt gesehen. Oft konzentrieren sich verteilungspolitische Probleme jedoch stärker auf Personen im unteren Bereich der Einkommensverteilung. Dies erklärt die Verwendung von Armutsmessgrößen (EQ2). Besonders besorgniserregend ist diesbezüglich häufig die Situation von Kindern, weil sie auf ihre materiellen Bedingungen keinen Einfluss haben und die Armut zudem längerfristige Konsequenzen für ihre Entwicklung hat. Daher ist es besonders sinnvoll, eine Armutsmessgröße aufzunehmen, die ausschließlich auf Kinder bezogen ist (EQ3).

Soziale Schutzsysteme sind ein wichtiges Instrument, mit dessen Hilfe die staatliche Politik auf Probleme der sozialen Gerechtigkeit reagiert. Alle OECD-Länder haben soziale Schutzsysteme entwickelt (oder sind dabei, es zu tun), die es in unterschiedlichem Ausmaß erlauben, Ressourcen innerhalb der Gesellschaft umzuverteilen und deren Mitglieder gegen diverse Risiken abzusichern. Der Indikator Angemessenheit der Mindestsicherungsleistungen (EQ4) bezieht sich auf Maßnahmen der staatlichen Politik, die speziell auf einige der am meisten benachteiligten Mitglieder der Bevölkerung abzielen. Diese Maßnahmen sind im Indikator öffentliche Sozialausgaben (EQ5) zusammengefasst. Der Indikator Gesamtsozialausgaben (EQ6) trägt zusätzlich auch Steuervorteilen und durch die Einkommensbesteuerung bedingten Rückflüssen an den Staat Rechnung.

Die Indikatoren für soziale Gerechtigkeit und Autonomie hängen eng miteinander zusammen. Gemeinsam betrachtet zeigen sie, wie die nationalen sozialen Sicherungssysteme einem chronischen politischen Dilemma gerecht zu werden versuchen, dem Interessenkonflikt zwischen der Bereitstellung angemessener Ressourcen auf der einen und der Nachhaltigkeit des Systems und Förderung der Autonomie des Einzelnen auf der anderen Seite.

\section{Liste der Indikatoren der sozialen Gerechtigkeit (EQ)}

\begin{tabular}{|c|c|c|c|}
\hline \multicolumn{2}{|c|}{ Sozialer Status } & \multicolumn{2}{|c|}{ Reaktion der Gesellschaft } \\
\hline EQ1. & Einkommensungleichheit & EQ4. & Angemessenheit von Mindestsicherungsleistungen \\
\hline EQ2. & Armut & EQ5. & Öffentliche Sozialausgaben \\
\hline EQ3. & Kinderarmut & EQ6. & Gesamtsozialausgaben \\
\hline SS1. & Beschäftigung & HE8. & Gesundheitsausgaben \\
\hline SS2. & Arbeitslosigkeit & & \\
\hline
\end{tabular}

Anmerkung: Kursiv gedruckt sind jene Indikatoren, die trotz ihres Erscheinens in einer anderen Unterkategorie auch für die Beurteilung der Ergebnisse im Bereich der sozialen Gerechtigkeit relevant sind.

\section{Gesundheit (HE)}

Es besteht ein enger Zusammenhang zwischen sozialen und gesundheitlichen Bedingungen. So hat denn auch der Anstieg des Lebensstandards und des Bildungsniveaus gemeinsam mit dem besseren Zugang zu Gesundheitsversorgungsleistungen und kontinuierlichen Fortschritten der medizinischen Technik zu einer signifikanten Verbesserung des Gesundheitszustands beigetragen, wie sie sich anhand des Indikators der Lebenserwartung messen lässt (HE1). Ein ebenso wichtiger Indikator, der die Messgrößen der Lebenserwartung zudem ergänzt, ist die Selbsteinschätzung des 
Gesundheitszustands (HE2). Die Fortschritte in Bezug auf die Lebenserwartung sind in erheblichem Maße auf Verbesserungen bei Indikatoren der Säuglingsgesundheit (HE3) wie der Säuglingssterblichkeit und dem Geburtsgewicht zurückzuführen. Fettleibigkeit (HE4) verringert die soziale und wirtschaftliche Funktionstüchtigkeit und ist ein Frühindikator für Gesundheitsprobleme und -kosten. Ein weiterer Indikator für den physischen Gesamtgesundheitszustand in der Kindheit, dem zunehmendes Augenmerk gilt, ist die erreichte Körpergröße von Erwachsenen (HE5). Im Gesundheitsbereich steht häufig die körperliche Gesundheit im Mittelpunkt, während die seelische Gesundheit (HE6) vielfach außer Acht gelassen wird. Zum Teil ist dies durch Mess- und Datenprobleme bedingt. Psychische Gesundheitsprobleme sind jedoch für den Einzelnen und die Gesellschaft mit hohen Kosten verbunden, und oft sind ein schlechter psychischer und physischer Gesundheitszustand miteinander verbunden.

Im Bereich Gesundheitsfragen ist eine Maßnahme die Bereitstellung verschiedener Arten von Langzeitpflegeleistungen für ältere Menschen in entsprechenden Einrichtungen und im gewohnten häuslichen Umfeld (HE7). Die Gesundheitsausgaben (HE8) sind ein allgemeinerer wichtiger Bestandteil des Politikinstrumentariums der Gesundheitssysteme, um auf Anliegen im Zusammenhang mit den gesundheitlichen Bedingungen einzugehen. Dennoch haben Gesundheitsprobleme ihre Wurzeln manchmal in anderen sozialen Bedingungen - wie Arbeitslosigkeit, Armut, unzulängliche Wohnverhältnisse -, die miteinander verknüpft sind, aber außerhalb der Reichweite der Gesundheitspolitik liegen. Darüber hinaus hängt die Effizienz gesundheitspolitischer Interventionen häufig stärker von anderen Merkmalen des Gesundheitssystems ab als vom Ausgabenniveau selbst, wie beispielsweise ein geringer Versicherungsschutz bzw. Zuzahlungen, die den Einzelnen davon abhalten können, medizinische Hilfe zu suchen. Ein breiteres Spektrum an Indikatoren für die gesundheitlichen Bedingungen und Interventionen findet sich in OECDGesundheitsdaten und dem dazugehörigen Begleitband Gesundheit auf einen Blick, der im zweijährigen Turnus veröffentlicht wird.

\section{Liste der Gesundheitsindikatoren (HE)}

\begin{tabular}{llll}
\hline \multicolumn{2}{l}{ Sozialer Status } & \multicolumn{2}{l}{ Reaktion der Gesellschaft } \\
\hline HE1. & Lebenserwartung & HE5. & Körpergröße \\
HE2. & Selbsteinschätzung des Gesundheitszustands & HE6. & Seelische Gesundheit \\
HE3. & Gesundheitszustand von Säuglingen & HE7. & Langzeitpflegebedürttige \\
HE4. & Fettleibigkeit & HE8. & Gesundheitsausgaben \\
C04. & Suizide & EQ4. & Angemessenheit von Mindestsicherungsleistungen \\
& & EQ5. & Öffentliche Sozialausgaben \\
& & EQ6. & Gesamtsozialausgaben \\
\hline
\end{tabular}

Anmerkung: Kursiv gedruckt sind jene Indikatoren, die trotz ihres Erscheinens in einer anderen Unterkategorie auch für die Beurteilung der Gesundheitsergebnisse relevant sind.

\section{Sozialer Zusammenhalt (CO)}

Die Förderung des sozialen Zusammenhalts ist in vielen OECD-Ländern ein wichtiges Ziel der Sozialpolitik. Auf Grund des Fehlens einer allgemein anerkannten Definition des Begriffs ist die Erstellung geeigneter Indikatoren besonders schwierig. Das in Gesellschaft auf einen Blick gewählte Konzept besteht darin, den sozialen Zusammenhalt anhand von Indikatoren zu beurteilen, die beschreiben, bis zu welchem Grad die Bürger am gesellschaftlichen Leben teilhaben und mit den Aktivitäten ihres täglichen Lebens 
zufrieden sind, Informationen über verschiedene Pathologien und Bedingungen liefern, die von ihnen betroffene Personen der Gefahr sozialer Ausgrenzung aussetzen, bzw. Aufschluss über das Ausmaß der sozialen Konflikte geben.

Erhebungsdaten über die subjektive Lebenszufriedenheit (CO1) liefern wichtige direkte Messgrößen für das Wohlbefinden des Einzelnen und den Zusammenhalt der Gesellschaft insgesamt. Der Arbeitsplatz ist ein Umfeld, in dem die meisten Menschen einen erheblichen Teil ihres Lebens verbringen. Die Arbeitszufriedenheit (CO2) ist daher eine wichtige Teildimension des Wohlbefindens und somit ein Indikator für den sozialen Zusammenhalt in einem entscheidenden Lebensbereich. Die Kriminalität ist eine Messgröße des sozialen Verfalls, und auch die kriminalitätsbedingte Viktimisierung (CO3) dürfte das Vertrauen in die Gesellschaft untergraben, das im Hinblick auf den sozialen Zusammenhalt eine wichtige Rolle spielt. Suizide (CO4) können als die endgültige Abkehr des Einzelnen von der Gesellschaft betrachtet werden, wenn auch soziale Bindungen nicht ausreichen, ihn davon abzuhalten, sich das Leben zu nehmen. Für jüngere Mitglieder der Gesellschaft ist die Schule vielleicht das wichtigste Umfeld außerhalb der Familie. Aggressives Verhalten (Bullying) in der Schule (CO5) ist ein wichtiger negativer Indikator für den sozialen Zusammenhalt im Schulumfeld. Ein weiterer Indikator für den sozialen Zusammenhalt ist bei Jugendlichen das Risikoverhalten (CO6). Ein gewisses Maß an Risikoverhalten ist natürlich normal und gehört, wenn es in bestimmte Richtungen geht, mit zum Erwachsenwerden. Gesellschaften, in denen der Zusammenhalt zwischen den Generationen fehlt, könnten jedoch ungewollt eine hohe Rate an Risikoverhalten erzeugen, das nicht in die erwünschte Richtung geht.

Über diese Indikatoren des sozialen Status hinaus lässt sich die Existenz verschiedener Gruppen und Familien in der Gesellschaft, die der speziellen Gefahr der sozialen Ausgrenzung ausgesetzt sind, auch durch Kontextindikatoren beleuchten. Letztlich sollte beachtet werden, dass es sehr viel schwieriger ist, geeignete Indikatoren für Maßnahmen zu finden, die bei Problemen des sozialen Zusammenhalts getroffen werden. Alle Maßnahmen, die für andere Dimensionen der Sozialpolitik (Autonomie, soziale Gerechtigkeit und Gesundheit) von Bedeutung sind, können den sozialen Zusammenhalt ebenfalls beeinflussen.

\begin{tabular}{llll}
\multicolumn{2}{l}{ Liste der Indikatoren } & für den sozialen Zusammenhalt (CO) \\
\hline Sozialer Status & \multicolumn{3}{l}{ Reaktion der Gesellschaft } \\
\hline C01. & Lebenszufriedenheit & CO4. & Suizide \\
C02. & Arbeitszufriedenheit & C05. & Bulllying \\
C03. & Kriminalitätsbedingte Viktimisierung & CO6. & Risikoverhalten \\
SS2. & Arbeitslosigkeit & & \\
EQ1. & Einkommensungleichheit & EQ5. & Öffentliche Sozialausgaben \\
EQ2. & Armut & EQ6. & Gesamtsozialausgaben \\
EQ3. & Kinderarmut & & \\
HE6. & Seelische Gesundheit & HE8. & Gesundheitsausgaben
\end{tabular}

Anmerkung: Kursiv gedruckt sind jene Indikatoren, die trotz ihres Erscheinens in einer anderen Unterkategorie auch für die Beurteilung der Ergebnisse des sozialen Zusammenhalts von Bedeutung sind. 


\section{Was bietet diese Veröffentlichung}

Für jeden in den Kapiteln 4 bis 8 dieses Berichts behandelten Bereich gibt zu Beginn ein Kasten unter der Überschrift „Definition und Messung“ die Definition des oder der einschlägigen Indikatoren sowie Informationen zu Messproblemen. Der Schwerpunkt liegt zudem auf Problemen der Vergleichbarkeit zwischen den Ländern. Einige Indikatoren sind nicht präzise miteinander vergleichbar. In den Fällen, in denen die Vergleichbarkeit ein potenzielles Problem darstellt, enthält der Kasten Informationen über diesen Sachverhalt. Nach dem Kasten zu „Definition und Messung“ wird dann das Hauptthema behandelt. In der Regel wird zunächst auf die länderspezifischen Unterschiede eingegangen, danach werden dann die Entwicklungen im Zeitverlauf für den OECD-Durchschnitt und eine Auswahl interessanter länderspezifischer Fälle untersucht. Soweit möglich werden demografische und sozioökonomische Aufschlüsselungen vorgenommen. Die entsprechenden Daten werden in Form von Abbildungen und Tabellen dargestellt, mit ausgewählten Verweisen „auf weitere Informationen“ und die Titel der Veröffentlichungen, denen die Indikatoren entnommen wurden.

Um die wichtigsten Daten in komprimierter Form vorzustellen, konnten in dieser Veröffentlichung nicht alle Dimensionen der erfassten Indikatoren berücksichtigt werden. Die jedem Indikator zu Grunde liegenden Daten finden sich auf der OECD-Website (www.oecd.org/els/social/indicators/SAG) oder sind über die StatLinks abrufbar (wo auch die Daten für andere Länder verfügbar sind). 


\section{Definition und Messung}

Unter den verschiedenen Messgrößen des Systems der Volkswirtschaftlichen Gesamtrechnungen (SNA) ist das Pro-Kopf-Nettonationaleinkommen (NNE) der am besten geeignete Indikator für den Vergleich des wirtschaftlichen Wohlergehens in verschiedenen Ländern. Das Nettonationaleinkommen ist definiert als das Bruttoinlandsprodukt (BIP) zuzüglich Nettoerwerbs- und Vermögenseinkommen aus dem Ausland, abzüglich der Wertminderung des Anlagevermögens (Wohnungen, Gebäude, Maschinen, Transportmittel und materielle Infrastruktur) durch Verschleiß, Abnutzung und Alterung. Schätzungen des Pro-Kopf-NNE sind jedoch mit größerer Unsicherheit behaftet als solche des Pro-Kopf-BIP, des am häufigsten verwendeten Indikators des Nationaleinkommens. Dies erklärt sich aus den Schwierigkeiten, die bei der Messung der internationalen Einkommensströme und der Wertminderung des Anlagevermögens auftreten.

Für die Zwecke des Ländervergleichs werden die NNE-Werte in Landeswährung anhand von Kaufkraftparitäten (KKP) in eine gemeinsame Messgröße umgerechnet. Die Kaufkraftparitäten entsprechen dem Betrag in nationaler Währung, der in den einzelnen Ländern erforderlich ist, um den gleichen Warenund Dienstleistungskorb zu erstehen, der in den Vereinigten Staaten für 1 US-\$ zu haben ist. Die KKPSchätzungen werden von OECD und Eurostat gemeinsam auf der Grundlage eines Vergleichs der Preise von rd. 2500 Posten in verschiedenen Ländern berechnet (Schreyer und Koechlin, 2002). Zur Berechnung des Pro-Kopf-NNE wird das NNE durch die Zahl der Einwohner des jeweiligen Landes geteilt, was sowohl die in Privathaushalten als auch die in Einrichtungen lebende Bevölkerung umfasst. Das NNE ebenso wie die KKP unterliegen statistischen Fehlern, weshalb Unterschiede zwischen den Ländern von bis zu 5\% nicht als signifikant zu betrachten sind.

Das Pro-Kopf-Nationaleinkommen betrug 2006 im OECDDurchschnitt etwa 26000 US-\$. In drei Ländern überstieg es in diesem Jahr 35000 US-\$ - in Luxemburg, Norwegen und den Vereinigten Staaten -, während es in acht Ländern unter 20000 US-\$ und in zwei Ländern (Mexiko und der Türkei) bei etwa 11000 US-\$ lag (Abbildung GE1.1) (die Zahl für Mexiko bezieht sich auf 2004).
Im Durchschnitt der 17 OECD-Länder, für die Daten zum realen Nettonationaleinkommen vorliegen, ist das ProKopf-NNE seit der Jahrtausendwende um 2,6\% jährlich gestiegen (Abbildung GE1.2). In der Slowakischen Republik belief sich der Anstieg auf über 5\% und in Griechenland auf fast $4,5 \%$ jährlich. In Italien und Deutschland fielen die jahresdurchschnittlichen Zuwachsraten des Pro-Kopf-NNE mit $1 \%$ hingegen bescheiden aus. Zwischen den Wachstumsraten im Zeitraum 2000-2006 zum einen und der Höhe des NNE im Jahr 2000 zum anderen sind kaum Korrelationen zu erkennen.

OECD-Länder mit höherem durchschnittlichem Pro-KopfNNE geben in der Tendenz einen größeren Prozentsatz ihres Nationaleinkommens für den sozialen Schutz aus. Dieser Zusammenhang ist indessen nicht sehr stark und erklärt sich zu einem großen Teil aus den Ausreißerpositionen Mexikos, der Türkei und Koreas (Abbildung GE1.3). Österreich, Dänemark, Frankreich und Schweden geben mehr für soziale Bereiche aus als angesichts ihres Pro-KopfEinkommens zu erwarten wäre. In Mexiko, Korea, der Türkei sowie in geringerem Maße den Vereinigten Staaten sind die Sozialausgaben im Verhältnis zum Pro-Kopf-Einkommen hingegen geringer. Es gibt mehrere mögliche Erklärungen für diese Unterschiede. Mit einigen Sozialausgaben werden von Dritten erbrachte Dienstleistungen bezahlt, z.B. Krankenpflege und Kinderbetreuung. Insofern der Verdienst dieser Dienstleistungserbringer parallel zum Gesamtverdienst der Bevölkerung steigt, ohne aber mit einem entsprechenden Produktionsanstieg verbunden $\mathrm{zu}$ sein, erhöht sich der Anteil der Sozialausgaben (Arjona et al., 2001). Außerdem können die vom Wohlfahrtsstaat erbrachten Leistungen stärker einkommensabhängig sein als andere Güter und Dienstleistungen.

\section{Weitere Informationen}

Arjona, R., M. Ladaique und M. Pearson (2001), „Growth, Inequality and Social Protection“, Labour Market and Social Policy Occasional Paper, No. 51, OECD, Paris.

OECD (2008), National Accounts, OECD, Paris.

Schreyer, P. und F. Koechlin (2002), „Purchasing Power Parities - Measurement and Uses“, OECD Statistics Brief, No. 3, OECD, Paris, März.

\section{Anmerkungen zu den Abbildungen}

Abbildung GE1.2: Viele Länder erstellen keine Preisdeflatoren für das NNE. Die Daten zum realen NNE-Wachstum beziehen sich daher nur auf 17 Länder.

NNE: Nettonationaleinkommen. 
GE1.1. Das Nettonationaleinkommen pro Kopf variiert in den OECD-Ländern zwischen 11000 US-\$ und 55000 US-\$

Pro-Kopf-NNE in US-\$ zu jeweiligen Preisen und KKP von 2006, OECD-Durchschnitt = 26500 US-\$

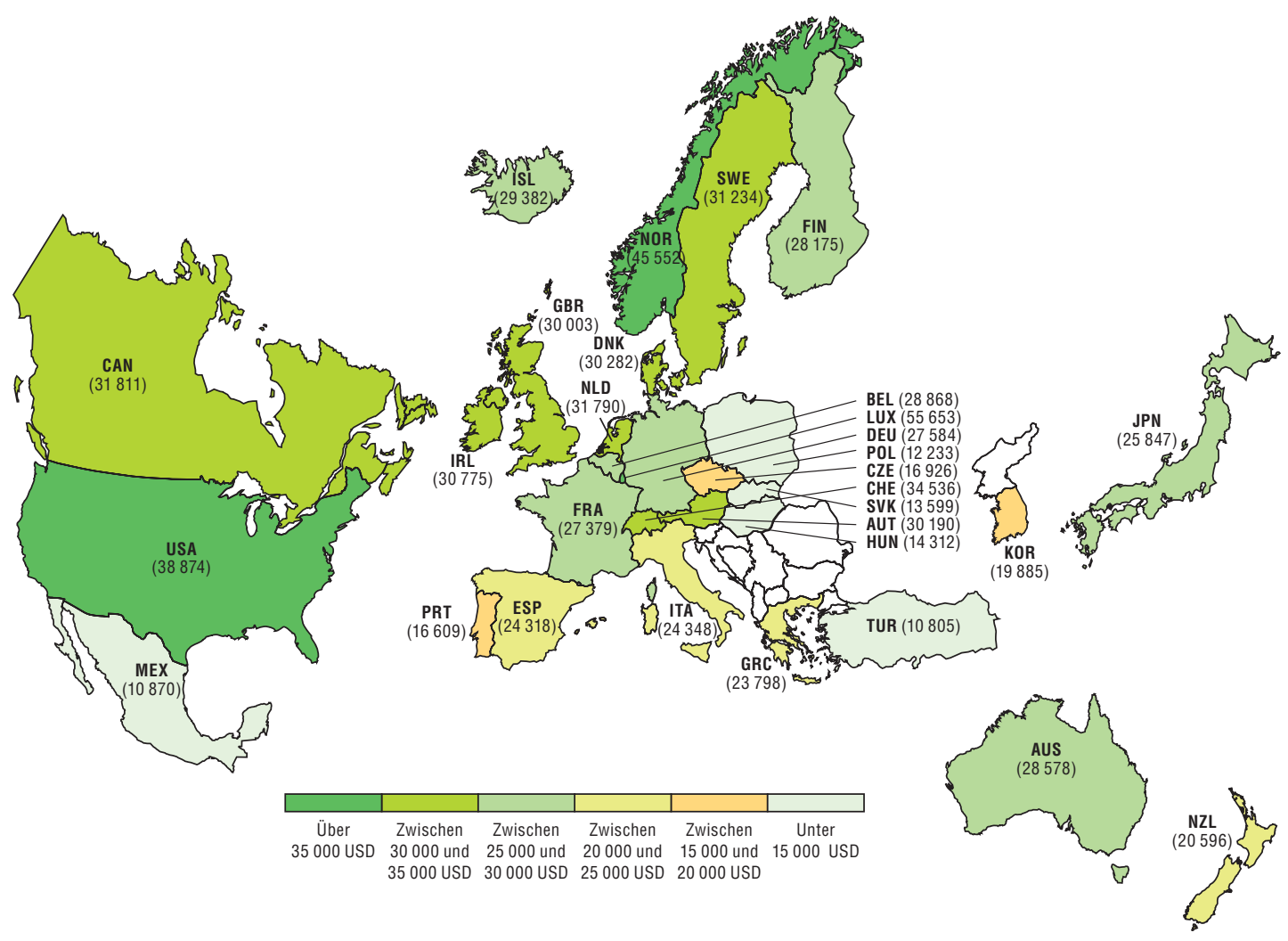

GE1.2. Das NNE-Wachstum schwankte zuletzt zwischen $1 \%$ und $5 \%$ jährlich

Reales jahresdurchschnittliches Wachstum des Pro-Kopf-NNE im Zeitraum 2000-2006, in \%

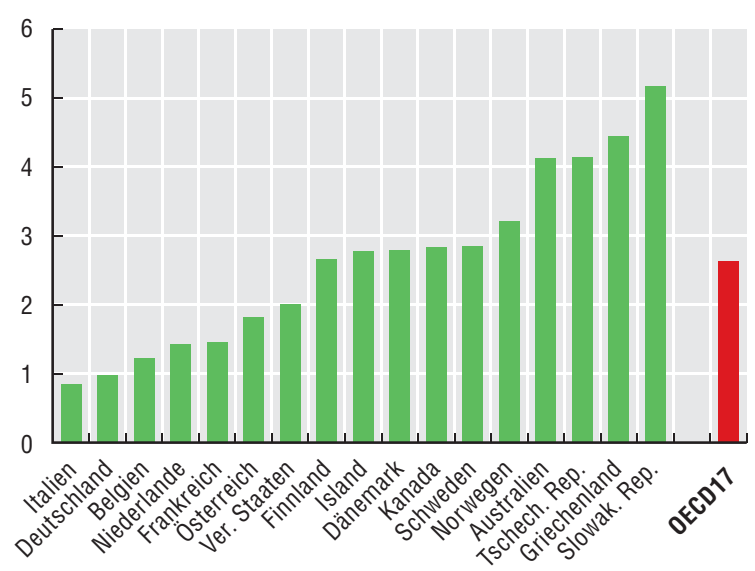

Quelle: OECD Annual National Accounts (www.oecd.org/statistics/ national-accounts) und Social Expenditure Database (www.oecd.org/ els/social/expenditure).
GE1.3. Länder mit höherem Nettonationaleinkommen verwenden einen größeren Prozentsatz davon für öffentliche Sozialausgaben

Öffentliche Sozialausgaben in \% des NNE und Pro-Kopf-NNE in US-\$ und KKP, 2005

Öffentliche Sozialausgaben in \% des NNE

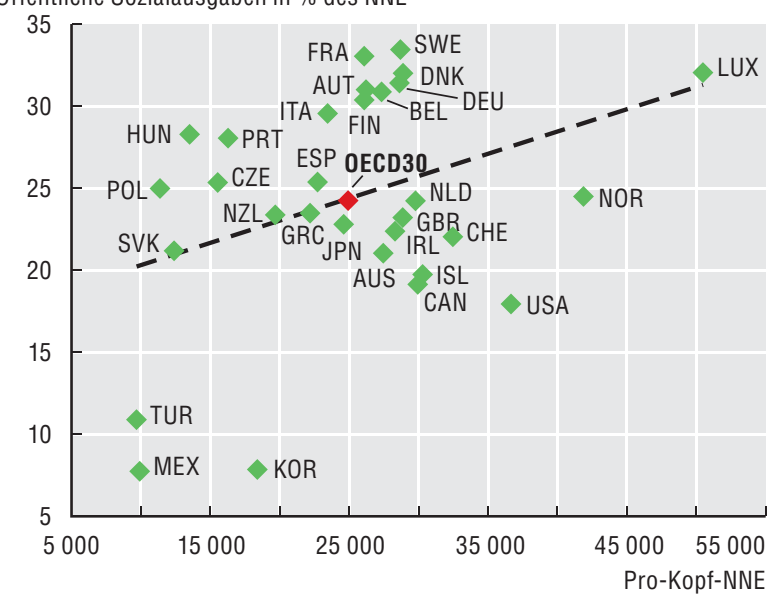

StatLink क्ञाs http://dx.doi.org/10.1787/550002185115 


\section{Definition und Messung}

Die zusammengefasste Geburtenziffer ist die Gesamtzahl der Kinder, die jede Frau bis zum Ende ihres gebärfähigen Alters zur Welt bringen würde, wenn ihre Geburtenwahrscheinlichkeit in jedem Alter der jeweiligen altersspezifischen Geburtenziffer entspräche. Sie wird durch Addition der über Fünfjahreszeiträume berechneten altersspezifischen Geburtenziffern ermittelt. Ohne Migration und bei unveränderten Sterberaten ist bei einer zusammengefassten Geburtenziffer von 2,1 Kindern je Frau (Bestandserhaltungsniveau) gewährleistet, dass die Bevölkerungszahl weitgehend stabil bleibt.

Das durchschnittliche Alter bei Geburt des ersten Kindes errechnet sich aus dem Mittelwert der verschiedenen Altersgruppen, gewichtet mit der Geburtenziffer der jeweiligen Altersgruppen. Der Anteil der nichtehelichen Geburten entspricht der Zahl der in einem bestimmten Jahr außerhalb der Ehe geborenen Kinder im Verhältnis zur Gesamtzahl der Lebendgeburten des gleichen Jahres.

Die Daten stammen in der Regel aus standesamtlichen Registern, Einwohnerregistern oder anderen amtlichen Quellen und wurden entsprechend den Empfehlungen der Vereinten Nationen und von Eurostat harmonisiert. Die Türkei bildet eine Ausnahme, dort werden die Geburtenziffern auf der Basis von Erhebungsdaten ermittelt.

Die meisten OECD-Länder verzeichnen niedrige Geburtenziffern. Dafür gibt es eine Reihe von Gründen, darunter veränderte Lebensgewohnheiten, Arbeitsmarktunsicherheit, Wohnungsprobleme, unerschwingliche, fehlende oder schlechte Kinderbetreuungsdienste, ein gestiegenes Bildungsniveau der Frauen, weniger Benachteiligung der Frauen in der Arbeitswelt sowie ein Unvermögen seitens der Politik, Familien in geeigneter Weise dabei zu helfen, Kindererziehung und Beruf miteinander zu verbinden. Viele dieser Probleme können den langfristigen Rückgang der Geburtenziffern erklären, der in den OECD-Ländern zu beobachten ist (D'Addio und Mira D'Ercole, 2005).

In den meisten OECD-Ländern liegen die Geburtenziffern unter dem Bestandserhaltungsniveau. 2006 betrug die durchschnittliche Geburtenziffer im OECD-Raum 1,65. Bei den wenigen Ländern, in denen die Geburtenziffern über dem Bestandserhaltungsniveau liegen, handelt es sich um Mexiko und die Türkei $(2,2)$ sowie Island und die Vereinigten Staaten (etwa 2,1).

Der Rückgang der Geburtenziffern kann die Politik vor Herausforderungen stellen. Sie muss insbesondere Antworten auf Fragen finden wie den Rückgang der Zahl der Personen, die sich um pflegebedürftige ältere Familienangehörige kümmern können, die wachsende Steuerbelastung der Personen im Erwerbsalter, die Renten und Gesundheitsversorgung finanzieren müssen, die Alterung der Erwerbsbevölkerung sowie die Abnahme der Inlandsersparnis.
Seit 2002 ist wieder eine leichte Zunahme der Geburtenziffern zu verzeichnen. Lag die durchschnittliche Geburtenziffer im OECD-Raum 2002 noch bei 1,60, war sie 2006 auf 1,65 angestiegen (Abbildung GE2.1). Diese Zunahme ist in 17 Ländern zu beobachten, wobei der stärkste Anstieg im Vereinigten Königreich, in Frankreich, Schweden, Spanien und in der Tschechischen Republik stattfand. Eine mögliche Erklärung könnte die Zunahme der Zahl der Geburten in der Altersgruppe der über 30-Jährigen sein, die ihrerseits auf die Verschiebung des Alters der Frauen bei der Geburt des ersten Kindes zurückzuführen ist (Sardon, 2006). Politikmaßnahmen wie die Ausdehnung der Unterstützung für Familien und erwerbstätige Mütter könnten ebenfalls eine Rolle gespielt haben.

Frauen sind bei der Geburt des ersten Kindes heute älter als in der Vergangenheit. Das durchschnittliche Alter bei der ersten Geburt ist seit 1970 im Durchschnitt pro Jahrzehnt um ein Jahr gestiegen (Tabelle GE2.2). Abgesehen davon, dass sie zu einem Rückgang der Geburtenziffern führt, hat die Erhöhung des durchschnittlichen Erstgebärendenalters noch andere dauerhafte Konsequenzen: Mit ihr steigt die Wahrscheinlichkeit, dass Frauen kinderlos bleiben oder weniger Kinder haben als sie eigentlich möchten; zudem erhöhen sich damit die Gesundheitsrisiken für Mutter und Kind.

Das durchschnittliche Heiratsalter der Frauen ist gestiegen. In den Ländern, die 2005 höhere Geburtenziffern verzeichneten, ist auch der Anteil der außerehelichen Geburten gestiegen (Abbildung GE2.3). Im Ländervergleich sind hier jedoch große Unterschiede festzustellen. In Ländern wie Japan und Korea sowie in mehreren südeuropäischen Ländern, wo noch immer ein enger Zusammenhang zwischen Ehe und Kinderkriegen besteht, sind z.B. sowohl die Geburtenziffern als auch der Anteil der außerehelichen Geburten gering.

\section{Weitere Informationen}

D'Addio, A.C. und M. Mira d'Ercole (2005), „Trends and Determinants of Fertility Rates in OECD Countries: the Role of Policies“, OECD Social, Employment and Migration Working Paper, No. 27, OECD, Paris.

OECD (2008), „SF3. Fertility rates“, OECD Family database, verfügbar unter www.oecd.org/els/social/family/database.

Sardon, J. (2006), „Recent Demographic Trends in Europe and the Other Developed Countries“, Population, Vol. 61, No. 3, S. 197-266.

\section{Anmerkungen zu den Abbildungen und Tabellen}

Tabelle GE2.2: Anmerkung 1: 1992 für Mexiko; Anmerkung 2: 2001 für Neuseeland; 2003 für Mexiko. Anmerkung 3: 2003 für Finnland, Griechenland, Spanien und das Vereinigte Königreich; 2002 für die Vereinigten Staaten; 2004 für Neuseeland; 2006 für Mexiko.

Abbildung GE2.3: Anmerkung 1: 2005 für Australien und 2007 für Belgien. 
GE2.1. Die Geburtenziffern liegen im OECD-Raum in der Regel unter dem Bestandserhaltungsniveau, seit Mitte der 1990er Jahre ist in einigen Ländern aber eine leichte Zunahme zu beobachten

Zusammengefasste Geburtenziffern 1970-2006

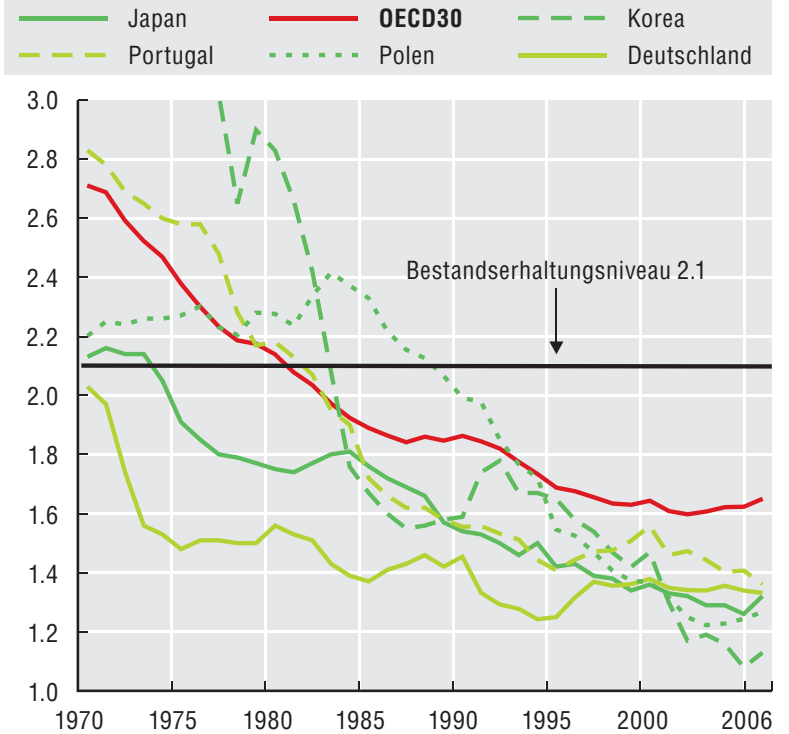

GE2.2. Das Durchschnittsalter der Mütter bei Geburt des ersten Kindes steigt

\begin{tabular}{|c|c|c|c|c|}
\hline & \multicolumn{4}{|c|}{ Durchschnittsalter bei Geburt des ersten Kindes } \\
\hline & 1970 & $1995^{1}$ & $2000^{2}$ & $2005^{3}$ \\
\hline Australien & 23.2 & 26.8 & . & 28.0 \\
\hline Belgien & 24.3 & 27.3 & .. & 27.4 \\
\hline Dänemark & 23.8 & 27.4 & 27.7 & 28.4 \\
\hline Deutschland & 24.0 & 27.5 & 28.2 & 28.1 \\
\hline Finnland & 24.4 & 27.2 & 27.4 & 27.9 \\
\hline Frankreich & 24.4 & 28.1 & 27.9 & 28.5 \\
\hline Griechenland & 25.0 & 26.6 & 27.5 & 28.5 \\
\hline Irland & .. & 27.3 & 27.6 & 28.5 \\
\hline Island & 21.3 & 25.0 & 25.5 & 26.3 \\
\hline Italien & 25.0 & 28.0 & .. & 28.7 \\
\hline Japan & 25.6 & 27.5 & 28.0 & 29.1 \\
\hline Korea & .. & .. & .. & 29.1 \\
\hline Luxemburg & 24.7 & 27.4 & 28.4 & 29.0 \\
\hline Mexiko & $\ldots$ & 20.9 & 21.0 & 21.3 \\
\hline Neuseeland & .. & . & 28.0 & 28.0 \\
\hline Niederlande & 24.8 & 28.4 & 28.6 & 28.9 \\
\hline Norwegen & $\ldots$ & 26.4 & 26.9 & 27.7 \\
\hline Österreich & .. & 25.6 & 26.4 & 27.2 \\
\hline Polen & 22.8 & 23.8 & 24.5 & 25.8 \\
\hline Portugal & .. & 25.8 & 26.5 & 27.4 \\
\hline Schweden & 25.9 & 27.2 & 27.9 & 28.7 \\
\hline Schweiz & 25.3 & 28.1 & 28.7 & 29.5 \\
\hline Slowak. Rep. & 22.6 & 23.0 & 24.2 & 25.7 \\
\hline Spanien & $\ldots$ & 28.4 & 29.1 & 29.3 \\
\hline Tschech. Rep. & 22.5 & 23.3 & 25.0 & 26.6 \\
\hline Ungarn & 22.8 & 23.8 & 25.1 & 26.7 \\
\hline Ver. Königreich & $\ldots$ & 28.3 & 29.1 & 29.8 \\
\hline Ver. Staaten & 24.1 & 24.5 & 24.9 & 25.1 \\
\hline 0ECD16 & 24.0 & 25.9 & 26.3 & 27.2 \\
\hline
\end{tabular}

Quelle: Eurostat und nationale Statistikämter.

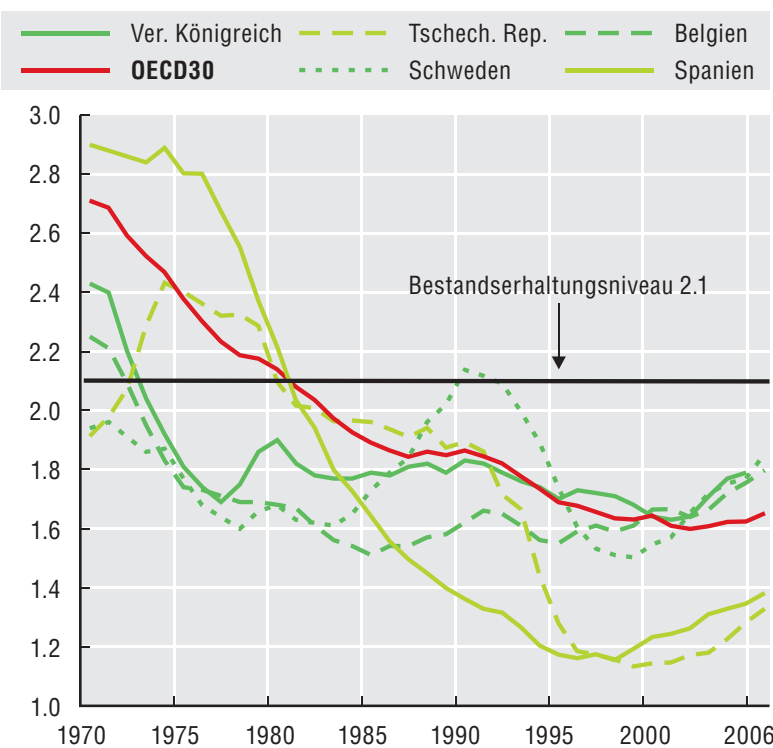

GE2.3. Positiver Zusammenhang zwischen Geburtenziffern und Anteil der außerehelichen Geburten

Anteil der außerehelichen Geburten ${ }^{1}$ und zusammengefasste Geburtenziffer, 2006

Zusammengefasste Geburtenziffer

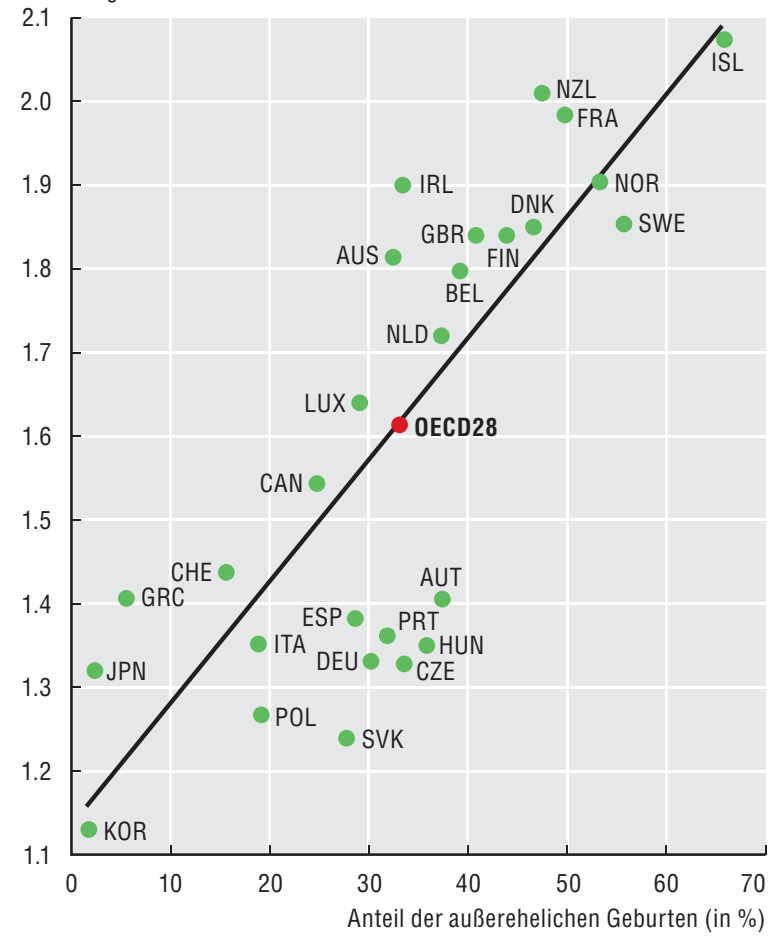

StatLink Alst http://dx.doi.org/10.1787/550015445534 


\section{Definition und Messung}

Geburtsland und Staatsangehörigkeit sind die beiden von den OECD-Ländern üblicherweise zur Definition der „zugewanderten“ Bevölkerung verwendeten Kriterien. Gemäß dem ersten Kriterium sind Zuwanderer in einem Land ansässige, aber dort nicht geborene Personen. Gemäß dem zweiten Kriterium sind Zuwanderer Gebietsansässige, die die Staatsangehörigkeit eines anderen Landes besitzen, aber u.U. im Aufnahmeland geboren sind. Im Ländervergleich festzustellende Unterschiede zwischen dem Umfang der im Ausland geborenen Bevölkerung und der ausländischen Bevölkerung hängen mit den jeweils geltenden Regelungen für den Erwerb der Staatsbürgerschaft zusammen. Die im Ausland geborene Bevölkerung ist im Allgemeinen wesentlich größer als die ausländische Bevölkerung. Weitere Informationen über Herkunft und Zusammensetzung der Zuwandererbevölkerung im OECD-Raum (nach Alter, Geschlecht, Bildungsniveau, Aufenthaltsdauer und Arbeitsmarktergebnissen) können der Publikation A Profile of Immigrant Populations in the 21st Century entnommen werden.

Der Wanderungssaldo entspricht der Summe der zuziehenden Ausländer und der aus dem Ausland zurückkehrenden Staatsangehörigen in einem gegebenen Jahr, abzüglich der Fortzüge von Aus- und Inländern im selben Jahr. Die Zuzugs- und Fortzugsdaten sind zwar im Allgemeinen nicht vergleichbar, in den Statistiken zum Wanderungssaldo, bei dem es sich um die Differenz zwischen Zuzügen und Fortzügen handelt, werden die Hauptursachen der Nichtvergleichbarkeit, d.h. die kurzfristigen Wanderungsbewegungen, aber in der Regel herausgerechnet. Der jährlich erscheinende International Migration Outlook der OECD liefert eine Gesamtanalyse der jüngsten Trends und Zuwanderungspolitiken in den OECD-Ländern.

2006 waren durchschnittlich rd. 12\% der Bevölkerung des OECD-Raums im Ausland geboren. Zwischen den OECDLändern bestehen erhebliche Unterschiede in Bezug auf den Umfang ihrer Zuwandererbevölkerung. In über der Hälfte der OECD-Länder beläuft sich der Anteil der Zuwandererbevölkerung an der Gesamtbevölkerung auf über $10 \%$ (Abbildung GE3.1). In Frankreich (8\%) und im Vereinigten Königreich (10\%) ist die Zuwandererbevölkerung relativ klein verglichen mit der Situation in den jeweiligen unmittelbaren Nachbarländern Spanien und Irland. Am höchsten ist der Anteil der im Ausland geborenen Bevölkerung mit 20\% und mehr in Australien, Kanada, Luxemburg, Neuseeland und der Schweiz. In Mexiko und der Türkei, zwei Ländern, die eine hohe Auswanderung verzeichnen, ist der Anteil der im Ausland geborenen Bevölkerung unerheblich. Im OECD-Raum ist ein rascher Anstieg des Anteils der im Ausland geborenen Bevölkerung zu beobachten. Der Anteil der im Ausland geborenen Bevölkerung hat sich seit dem Jahr 2000 um 2 Prozentpunkte erhöht. Einige Länder, namentlich Spanien (7\%), Irland (6\%) sowie Neuseeland und Österreich (beide $4 \%$ ), verzeichneten eine sehr starke Veränderung des Anteils der im Ausland geborenen Bevölkerung.

Im Durchschnitt erklärt sich bis zur Hälfte des in den OECD-Ländern in den letzten 10 Jahren verzeichneten Bevölkerungswachstums aus dem Wanderungssaldo. In einigen Ländern war der Großteil (z.B. in Spanien) bzw. das gesamte Bevölkerungswachstum (z.B. in Italien) der letzten zehn Jahre auf Migration zurückzuführen (Abbildung GE3.2). Am höchsten war der Wanderungssaldo in Irland, Luxemburg und Spanien.

Die Bruttozuwanderung hat im OECD-Raum in den letzten 15 Jahren zugenommen (Abbildung GE3.3). Hinter dem Wanderungssaldo verbirgt sich eine wesentlich stärkere Bruttozuwanderung bzw. -auswanderung. Viele OECDLänder stehen vor der Herausforderung der wirtschaftlichen und sozialen Integration der Zuwanderer und ihrer Kinder. Zugleich muss sich die Politik in einigen nichteuropäischen OECD-Ländern auch mit der Frage der Abwanderung junger, qualifizierter Arbeitskräfte - dem sogenannten „Braindrain“- auseinandersetzen.

\section{Weitere Informationen}

OECD (2007), Jobs for Immigrants (Vol. 1): Labour Market Integration in Australia, Denmark, Germany and Sweden, Paris.

OECD (2008a), International Migration Outlook, Paris.

OECD (2008b), A Profile of Immigrant Populations in the 21st Century: Data from OECD Countries, Paris.

OECD (2008c), Jobs for Immigrants (Vol. 2): Labour Market Integration in Belgium, France, Netherlands and Portugal, Paris.

\section{Anmerkungen zu den Abbildungen}

Abbildung GE3.3: EWR: Der Europäische Wirtschaftsraum umfasst die Europäische Union (hier EU15) sowie Island und Norwegen. 
GE3.1. Rascher Anstieg des Anteils der im Ausland geborenen Bevölkerung im OECD-Raum Im Ausland geborene Bevölkerung in Prozent der Gesamtbevölkerung, 2000 und 2006

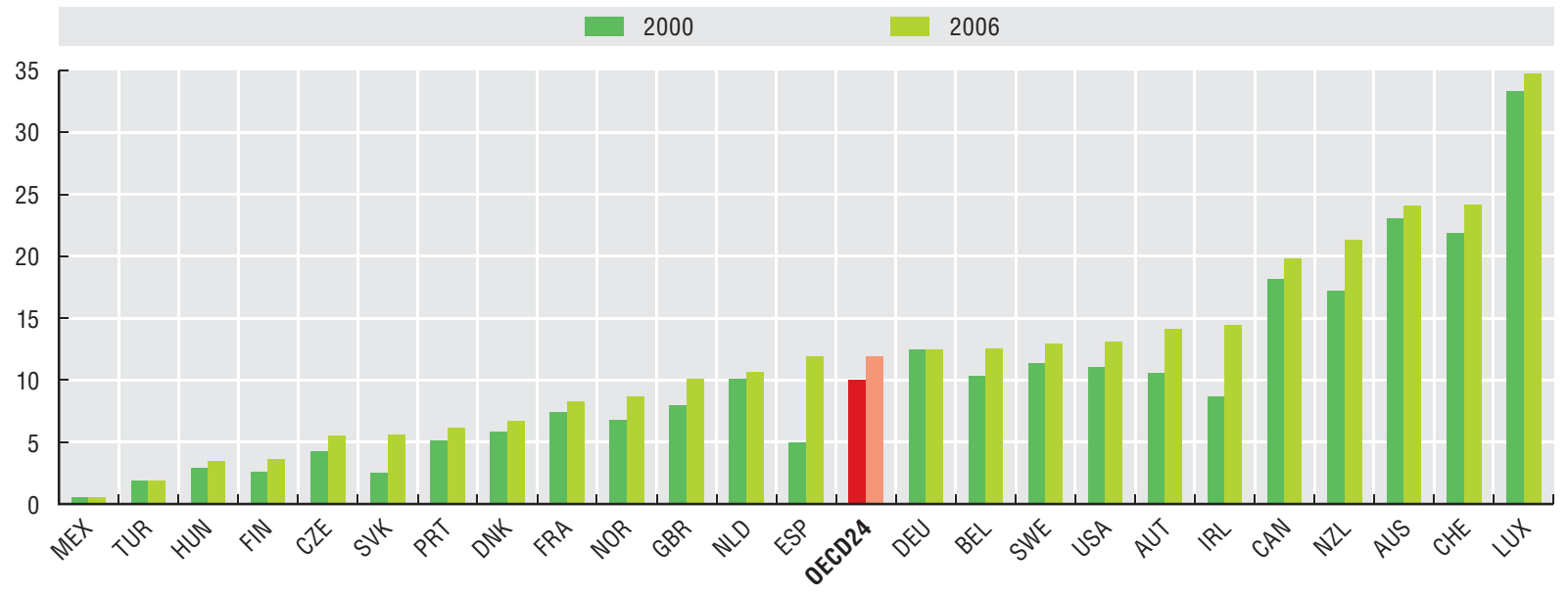

GE3.2. Bis zur Hälfte des Bevölkerungswachstums der letzten 10 Jahre erklärte sich im OECD-Raum aus dem Wanderungssaldo

Prozentuale Veränderung der Einwohnerzahlen im Zeitraum 1996-2006 und Beitrag des Wanderungssaldos

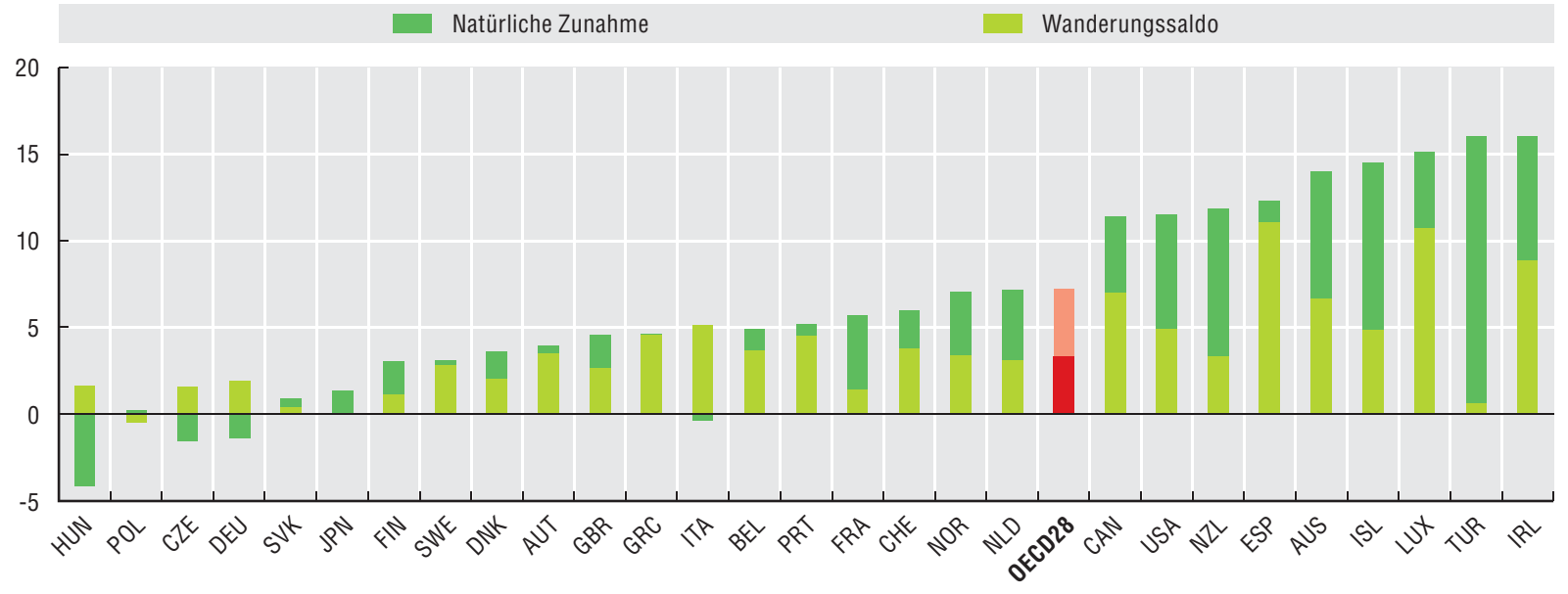

GE3.3. Die Bruttozuwanderung hat im OECD-Raum in den letzten 10 Jahren zugenommen

Zuzüge ausländischer Staatsangehöriger in ausgewählten OECD-Ländern, 1990-2006, 1990=100

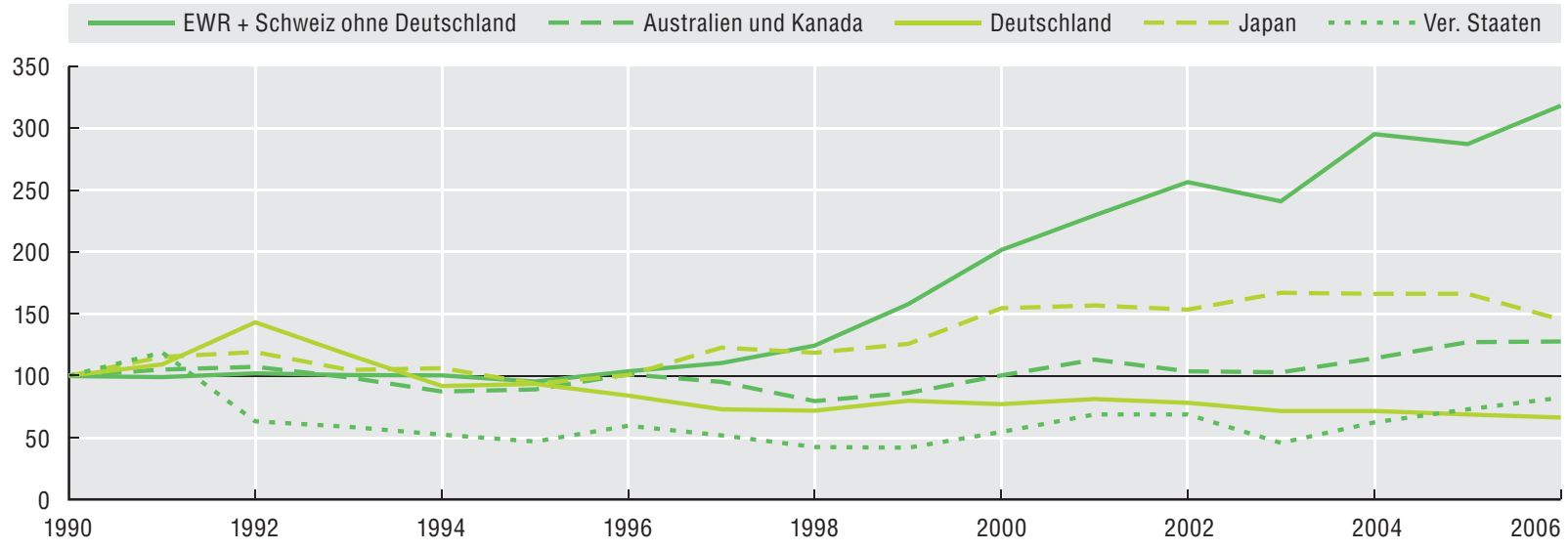

Quelle: OECD (2008), International Migration Outlook, Paris. 


\section{Definition und Messung}

Die rohe Heiratsrate entspricht der jährlichen Zahl der Eheschließungen im Verhältnis zur Gesamtbevölkerung. Bei der rohen Scheidungsrate handelt es sich um die Zahl der Ehen, die in einem Jahr rechtskräftig aufgelöst wurden, im Verhältnis zur Gesamtbevölkerung. Die Daten zu Eheschließungen und Scheidungen stammen aus amtlichen Registern. In den Heiratsraten nicht erfasst sind informelle Familiengründungen sowie andere Formen rechtskräftiger Lebensgemeinschaften. In den Scheidungsraten nicht berücksichtigt sind die Auflösungen solcher nicht erfassten Lebensgemeinschaften sowie Trennungen ohne Scheidung.

Die Heirats- und Scheidungsraten messen die Zugänge zur bzw. Abgänge aus der verheirateten Bevölkerung. Die Informationen zum Familienstand der Bevölkerung sind für 17 OECD-Länder dem European Social Survey 2006-2007 entnommen. In dieser Erhebung werden nicht nur zusammenlebende und rechtskräftig verheiratete Paare erfasst, sondern wird auch die Häufigkeit von eingetragenen Lebenspartnerschaften, die ein neues Merkmal der sozialen Landschaft mehrerer OECD-Länder sind, sowie von informellen eheähnlichen Gemeinschaften gemessen. Erfasst werden gleich- ebenso wie gemischtgeschlechtliche Paare, die zusammenleben oder eingetragene Lebenspartnerschaften eingegangen sind. Zwischen diesen Erhebungsdaten und anderen Informationsquellen zum Familienstand der Bevölkerung in einzelnen Ländern können Abweichungen auf Grund von Stichprobenvarianz und unterschiedlichen Beteiligungsquoten bestehen.

\begin{abstract}
Die Heiratsraten sind in den meisten OECD-Ländern gesunken. 2006 lag die rohe Heiratsrate im Durchschnitt von 26 OECD-Ländern bei 5,1 je 1000 Einwohner, über ein Drittel unter ihrem Niveau von 1970. Das Tempo des Rückgangs der Heiratsraten schwankt im OECD-Vergleich. In der Zeit nach 1990 war in der Tschechischen Republik, Korea und den Vereinigten Staaten ein sehr starker Rückgang festzustellen, während die Raten in Spanien und Schweden seit Ende der 1990er Jahre unverändert geblieben oder sogar gestiegen sind (Abbildung GE4.1).
\end{abstract}

Die Scheidungsraten haben in den meisten OECDLändern zugenommen. 2005 belief sich die rohe Scheidungsrate im Durchschnitt auf 2,3 je 1000 Einwohner, womit sie doppelt so hoch wie 1970 und um 0,2 Prozentpunkte höher war als im Jahr 2000. In der Zeit seit 1990 sind die Scheidungsraten in den Vereinigten Staaten gesunken, in Portugal, Polen, Spanien und Japan jedoch gestiegen (Abbildung GE4.2).

Die Ehe ist die bei weitem häufigste Form erwachsenen Zusammenlebens im OECD-Raum, ein großer Teil der
Bevölkerung lebt jedoch auch in eheähnlichen Gemeinschaften oder eingetragenen Lebenspartnerschaften (Tabelle GE4.3). In Portugal und der Schweiz sind über 60\% der Erwachsenenbevölkerung verheiratet, in Schweden jedoch nur $44 \%$. Die eingetragene Lebenspartnerschaft ist wo im Gesetz vorgesehen - wesentlich weniger beliebt als die Ehe; in Belgien (dort leben effektiv mehr Menschen in eingetragenen Lebenspartnerschaften als in informellen eheähnlichen Gemeinschaften), in den Niederlanden und in Ungarn beläuft sich ihr Anteil aber auf über 5\% der Erwachsenenbevölkerung. Die nordischen Länder, insbesondere Schweden, sowie Frankreich weisen einen hohen Anteil eheähnlicher Lebensgemeinschaften auf. In der Slowakischen Republik, Ungarn und Polen ist dieser Anteil hingegen sehr niedrig. Der Anteil der Alleinstehenden ist in Frankreich mit 28\% am geringsten und in Polen mit $42 \%$ am höchsten.

Männer sind bei der ersten Heirat zwar in der Regel älter als Frauen, dieses Altersgefälle zwischen Männern und Frauen ist in den einzelnen Ländern aber unterschiedlich groß. In Luxemburg und Österreich hat sich die Altersdifferenz zwischen Männern und Frauen bei der ersten Heirat im Zeitraum 1990-2003 erhöht (Abbildung GE4.4). In Irland ging der Anstieg des Heiratsalters der Frauen hingegen mit einer Abnahme des Altersgefälles zwischen Männern und Frauen bei der ersten Eheschließung einher.

\section{Weitere Informationen}

Dickmann, A. und K. Schmidheiny (2006), „The Intergenerational Transmission of Divorce - A Fifteen-Country Study with the Fertility and Family Survey“, Vorlage für 2004 Annual Meeting of the Population Association of America.

Morrison, D. und M. Coiro (1999), „Parental Conflict and Marital Disruption: Do Children Benefit When HighConflict Marriages are Dissolved?", Journal of Marriage and the Family, Vol. 61, No. 3, S. 626-637.

OECD (2008), „SF8. Marriage and divorce rates“, OECD Family Database, verfügbar unter www.oecd.org/els/social/family/ database.

Smock, P. (2004), „The Wax and Wane of Marriage: Prospects for Marriage in the 21st Century“, Journal of Marriage and the Family, Vol. 66, No. 4, S. 966-973.

Sobotka, T. und L. Toulemon (2008), „Changing Family and Partnership Behaviour: Common Trends and Persistent Diversity across Europe“, Demographic Research, Vol. 19, No. 6, S. 85-138.

\section{Anmerkungen zu den Abbildungen und Tabellen}

Tabelle GE4.3: Für Österreich liegen keine Daten zu anderen Kategorien vor als der Ehe. Fehlen Angaben zu eingetragenen Lebenspartnerschaften, heißt dies, dass diese Form des Zusammenlebens im jeweiligen Land nicht vorgesehen ist. 
GE4.1. Die Heiratsraten sind im Allgemeinen rückläufig Eheschließungen je 1000 Einwohner, 1970-2006

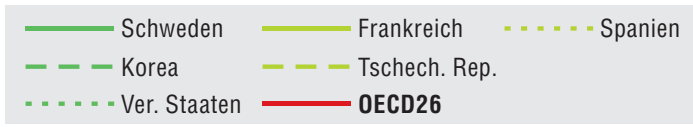

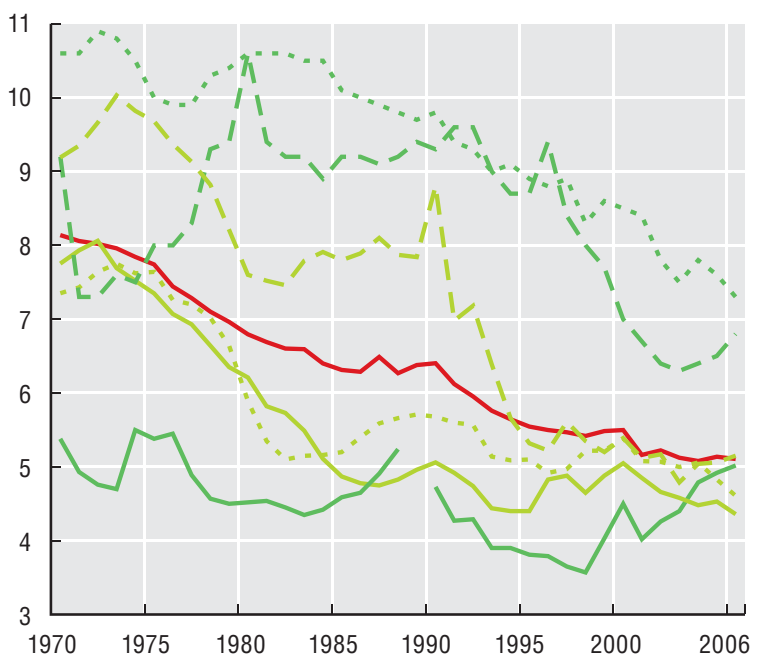

GE4.3. Ehe, eingetragene Lebenspartnerschaft oder informelle eheähnliche Gemeinschaft

In Prozent der Erwachsenenbevölkerung, 2006-2007

\begin{tabular}{|c|c|c|c|c|c|}
\hline & Verheiratet & $\begin{array}{c}\text { Eingetr. } \\
\text { Partner- } \\
\text { schaft }\end{array}$ & $\begin{array}{c}\text { Eheähnl. } \\
\text { Gemein- } \\
\text { schaft }\end{array}$ & $\begin{array}{l}\text { Allein- } \\
\text { stehend }\end{array}$ & Insgesamt \\
\hline Belgien & 54 & 7 & 6 & 34 & 100 \\
\hline Dänemark & 57 & 1 & 13 & 30 & 100 \\
\hline Deutschland & 54 & 1 & 8 & 36 & 100 \\
\hline Finnland & 51 & 0 & 15 & 34 & 100 \\
\hline Frankreich & 56 & 2 & 14 & 28 & 100 \\
\hline Irland & 55 & 0 & 7 & 37 & 100 \\
\hline Niederlande & 56 & 6 & 8 & 30 & 100 \\
\hline Norwegen & 50 & 3 & 14 & 33 & 100 \\
\hline Österreich & 52 & & & & 0 \\
\hline Polen & 56 & 0 & 2 & 42 & 100 \\
\hline Portugal & 62 & 0 & 4 & 34 & 100 \\
\hline Schweden & 44 & 1 & 21 & 35 & 100 \\
\hline Schweiz & 61 & 2 & 6 & 31 & 100 \\
\hline Slowak. Rep. & 56 & 5 & 1 & 39 & 100 \\
\hline Spanien & 55 & 3 & 4 & 38 & 100 \\
\hline Ungarn & 53 & 6 & 3 & 37 & 100 \\
\hline Ver. Königreich & 54 & 4 & 6 & 36 & 100 \\
\hline OECD17 & 54 & 3 & 8 & 35 & 100 \\
\hline
\end{tabular}

Quelle: Abb. GE4.1., GE4.2 und GE4.4. Eurostat und nationale Quellen; Abb. GE4.3 European Social Survey 2006/7
GE4.2. Die Scheidungsraten steigen im Allgemeinen Scheidungen je 1000 Einwohner, 1970-2006
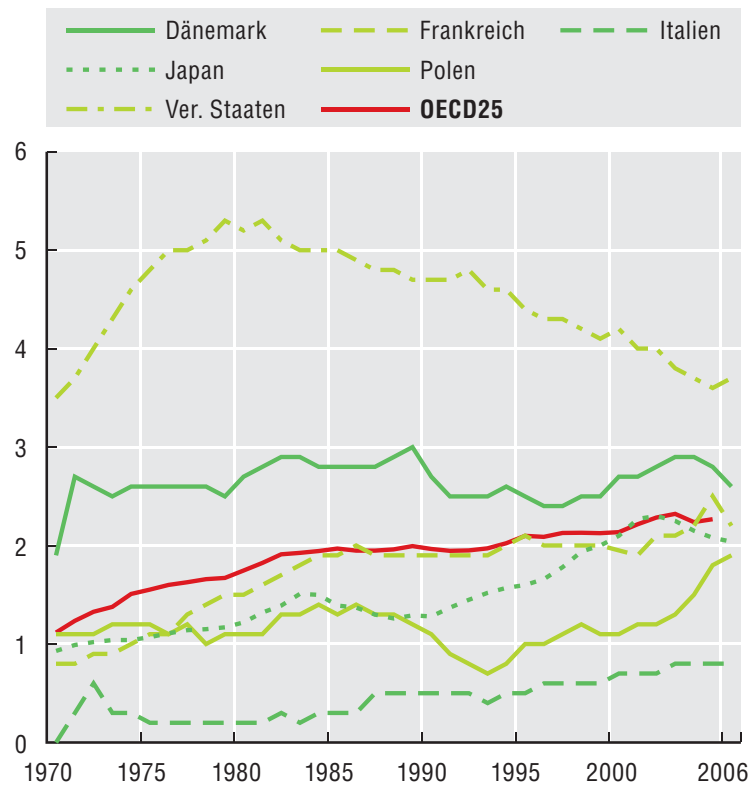

GE4.4. Altersgefälle zwischen Männern und Frauen bei der ersten Heirat

Veränderung der Differenz des durchschnittlichen Alters von Männern und Frauen bei der ersten Heirat, 1990-2003

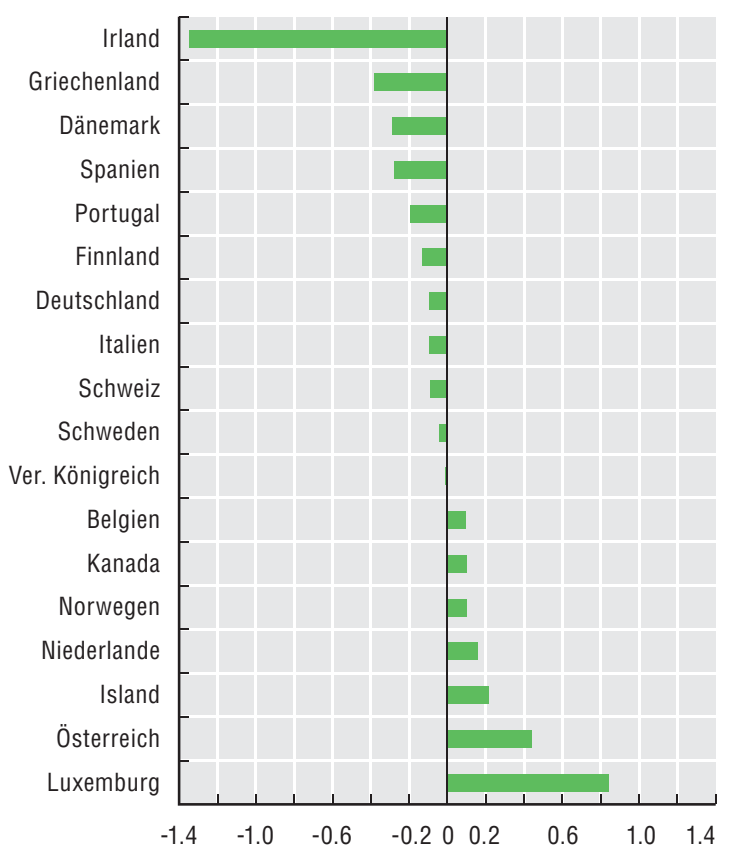

StatLink 에sस http://dx.doi.org/10.1787/550077543104 


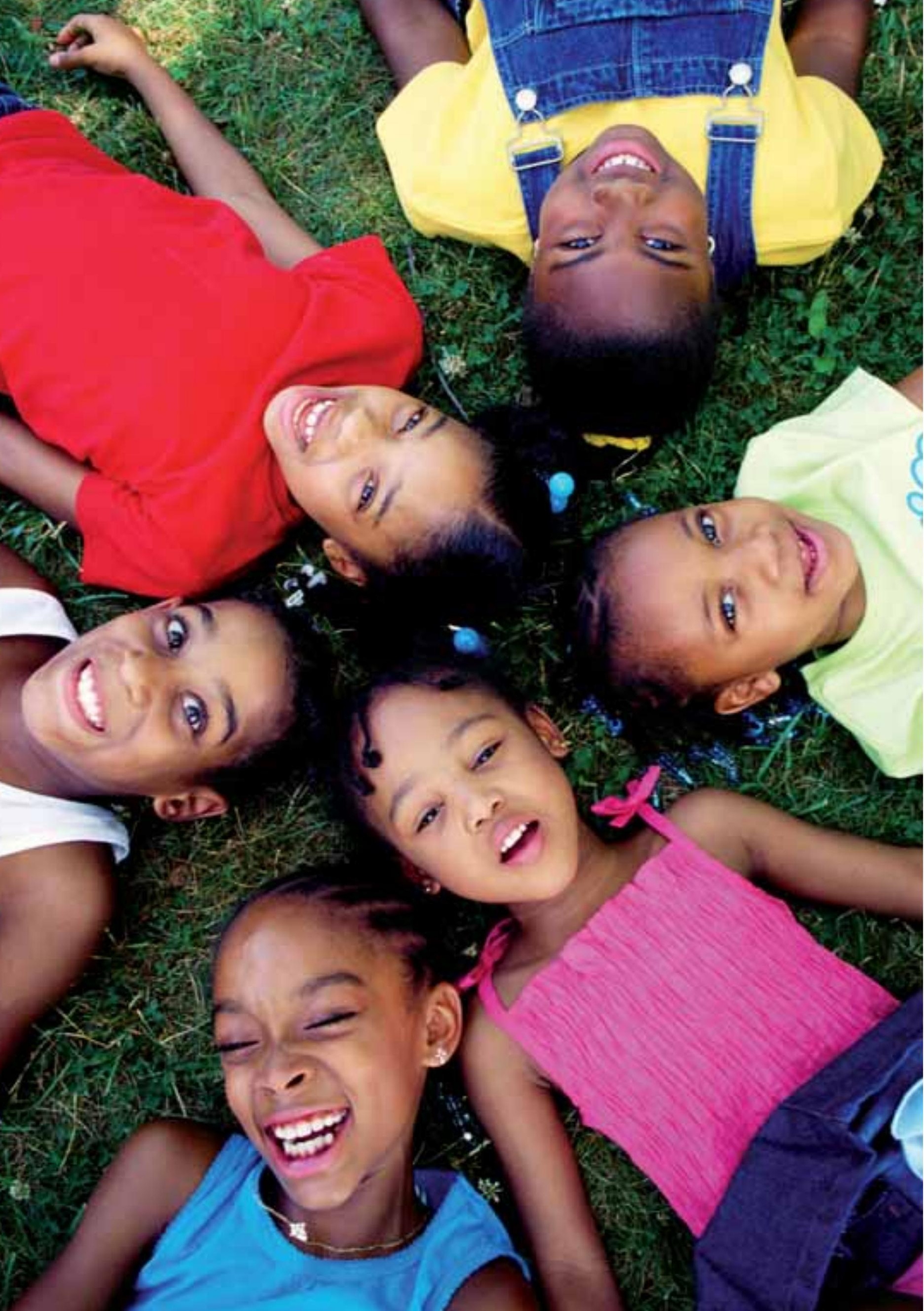





\section{Definition und Messung}

Eine Person gilt als erwerbstätig, wenn sie mindestens eine Stunde pro Woche gegen Entgelt oder im Rahmen einer selbstständigen oder mithelfenden Tätigkeit arbeitet oder sich in einem Arbeitsverhältnis befindet, das sie wegen Krankheit, Urlaub oder Arbeitskonflikten vorübergehend nicht ausübt. Die Daten aus Arbeitskräfteerhebungen der OECDLänder basieren auf dieser Arbeitsdefinition für eine jeweilige Berichtswoche. Der hier für die Beschäftigung herangezogene Basisindikator gibt den Anteil der beschäftigten Personen im erwerbsfähigen Alter zwischen 15 und 64 Jahren wieder. Diese Beschäftigungsquoten sind nach Alter, Geschlecht, Bildungsabschluss und Migrantenstatus aufgeschlüsselt.

Dieser Abschnitt enthält auch Daten über die Inzidenz der Teilzeitbeschäftigung in Prozent der Gesamtbeschäftigung. Der Begriff Teilzeiterwerbstätigkeit bezieht sich auf Personen, die gewöhnlich weniger als 30 Wochenstunden an ihrem Hauptarbeitsplatz verbringen. Die Daten erfassen nur Personen, die Fragen zu ihrer normalen Arbeitszeit beantworteten. Die OECD-Daten über die Beschäftigung stützen sich auf nationale Arbeitskräfteerhebungen.

Die Beschäftigungsquoten unter der Bevölkerung im erwerbsfähigen Alter sind in den meisten OECD-Ländern seit Mitte der 1990er Jahre gestiegen. In vielen OECDLändern lagen die Beschäftigungsquoten im Jahr 2007 höher als in den 1980er Jahren (Abbildung SS1.1). Die Zunahme der Beschäftigungsquoten seit 1995 betrug in Australien, Belgien, Finnland, Griechenland, Island, Italien, Kanada und den Niederlanden mehr als 5 Prozentpunkte und in Irland und Spanien sogar über 10 Prozentpunkte. Demgegenüber liegen die Beschäftigungsquoten in Polen und der Tschechischen Republik 1-3 Punkte niedriger als Mitte der 1990er Jahre und in der Türkei über 5 Punkte niedriger. Nach wie vor sind bei den Beschäftigungsquoten beträchtliche Unterschiede zwischen den Ländern auszumachen. Die Beschäftigungsquoten liegen in vierzehn Ländern, darunter Australien, Kanada, den Niederlanden, Schweden, dem Vereinigten Königreich und den Vereinigten Staaten bei $70 \%$ oder mehr und sinken in Italien, Polen, der Türkei und Ungarn unter 60\% (Tabelle SS1.3).

Die Wahrscheinlichkeit einer Erwerbstätigkeit liegt bei Jugendlichen und älteren Menschen wesentlich niedriger als bei Personen im Haupterwerbsalter (Abbildung SS1.2,
Tabelle SS1.3). Dieses Altersmuster findet sich in allen OECD-Ländern und spiegelt die Tatsache wider, dass sich viele junge Menschen im Bildungsprozess befinden, dass diejenigen Jugendlichen, die erwerbstätig sind, über wenig Arbeitserfahrung verfügen und dass viele ältere Menschen noch vor dem 65. Lebensjahr in den Ruhestand treten.

Bei Frauen liegt die Wahrscheinlichkeit einer Erwerbstätigkeit deutlich niedriger als bei Männern. Die Beschäftigungsquoten der Frauen blieben im Jahr 2007 um 16 Prozentpunkte hinter denen der Männer zurück. Es sind zwar Schwankungen zu beobachten, doch ist das Grundmuster in allen Ländern mehr oder minder gleich. In Griechenland, Italien, Mexiko und der Türkei erreichten die Beschäftigungsquoten der Frauen weniger als 50\%, in Dänemark, Island, Kanada, Norwegen, Schweden und der Schweiz dagegen über $70 \%$. Die Beschäftigungsquoten von Männern sind im Ländervergleich ähnlicher. Zu den Ländern mit geringen Unterschieden zwischen den Beschäftigungsquoten von Männern und Frauen gehören Finnland und Schweden. Eine große Lücke klafft in der Türkei und in Mexiko.

Die Wahrscheinlichkeit einer Erwerbstätigkeit ist bei Menschen mit geringer Schulbildung wesentlich niedriger. Dieses Muster ist in sämtlichen OECD-Ländern eindeutig und ohne Ausnahme vorhanden. Deshalb ist den Regierungen der OECD-Länder die Förderung der Bildung zur Sicherung der Autonomie ein großes Anliegen.

Bei Migranten ist die Wahrscheinlichkeit einer Erwerbstätigkeit etwas geringer als bei der im Inland geborenen Bevölkerung. Dennoch ist das durchschnittliche Gefälle bei der Beschäftigung in den OECD-Ländern im Vergleich zu den weiteren in Tabelle SS1.3 untersuchten Aspekten niedrig. In Italien, Luxemburg und Ungarn ist die Beschäftigungswahrscheinlichkeit bei Migranten sogar höher als bei im Inland Geborenen (um 7 oder mehr Prozentpunkte), genau umgekehrt ist es jedoch in den Niederlanden, Dänemark und Polen (um 15 oder mehr Prozentpunkte niedriger).

Die jüngste Finanzkrise wird wahrscheinlich die Beschäftigungsquoten Ende 2008 und Anfang 2009 nach unten drücken. Noch sind Ausmaß und Dauer eines Rückgangs nicht abzuschätzen.

\section{Anmerkungen zu den Abbildungen und Tabellen}

Abbildung SS1.2: Die Daten sind in aufsteigender Reihenfolge der weiblichen Beschäftigungsquote angeordnet. Daten für Geringqualifizierte, definiert als Personen, die nicht mindestens über einen Schulabschluss der Sekundarstufe II verfügen, stammen aus dem Jahr 2006.

Tabelle SS1.3: Anmerkung 1: 2006 nach Bildungsabschluss. Anmerkung 2: 2006 für Island, Kanada und die Vereinigten Staaten. 
SS1.1. Es gibt mehr Beschäftigte

Beschäftigungsquote, Personen zwischen 15 und 64 Jahren

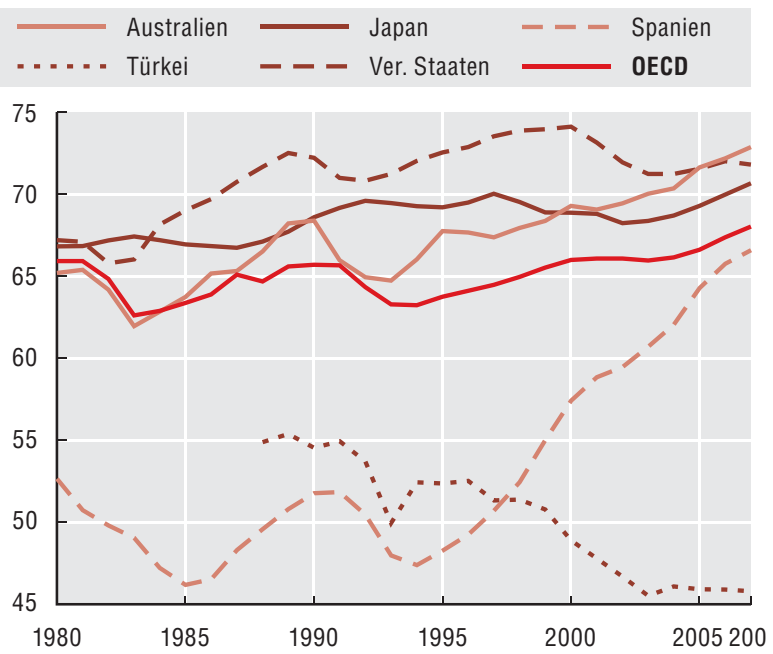

\section{SS1.2. Weiterhin erhebliche Unterschiede bei den Erwerbstätigenquoten zwischen soziodemografischen Gruppen}

Beschäftigungsquote nach Geschlecht und Altersgruppe, 2007

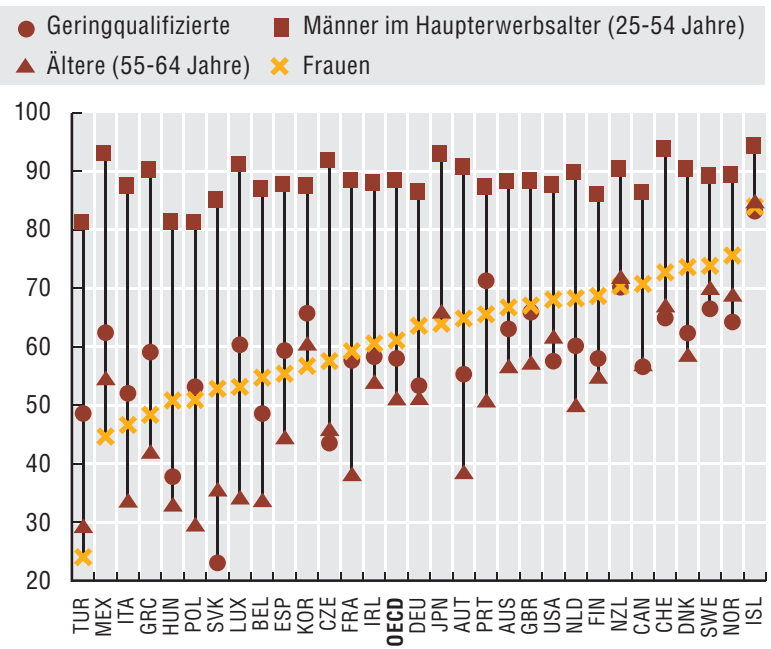

SS1.3 Beschäftigungsindikatoren, 2007

\begin{tabular}{|c|c|c|c|c|c|c|c|c|c|c|c|c|c|c|}
\hline & \multirow{3}{*}{$\begin{array}{l}\text { Beschäftigungs- } \\
\text { quote für die } \\
\text { Bevölkerung } \\
\text { zwischen } 15 \\
\text { und } 64 \text { Jahren }\end{array}$} & \multicolumn{10}{|c|}{ Beschäftigungsquote nach: } & \multirow{2}{*}{\multicolumn{3}{|c|}{$\begin{array}{c}\text { Inzidenz der } \\
\text { Teilzeitbeschäftigung } \\
\text { in \% der Beschäftigung }\end{array}$}} \\
\hline & & \multicolumn{3}{|c|}{ Alter } & \multicolumn{2}{|c|}{ Geschlecht } & \multicolumn{3}{|c|}{ Bildungsabschluss ${ }^{1}$} & \multicolumn{2}{|c|}{ Migrantenstatus $^{2}$} & & & \\
\hline & & 15-24 J. & $25-54 \mathrm{~J}$ & $55-64 \mathrm{~J}$. & Männer & Frauen & $\begin{array}{l}\text { Ohne } \\
\text { Sekundar- } \\
\text { stufe-II- } \\
\text { Abschluss }\end{array}$ & $\begin{array}{l}\text { Sekundar- } \\
\text { stufe-II- } \\
\text { Abschluss }\end{array}$ & $\begin{array}{c}\text { Tertiär- } \\
\text { abschluss }\end{array}$ & $\begin{array}{l}\text { Im Inland } \\
\text { geboren }\end{array}$ & $\begin{array}{c}\text { Im } \\
\text { Ausland } \\
\text { geboren }\end{array}$ & $\begin{array}{c}\text { Ins- } \\
\text { gesamt }\end{array}$ & Männer & Frauen \\
\hline Australien & 72.9 & 64.2 & 80.0 & 56.7 & 79.6 & 66.1 & 63.5 & 80.4 & 84.4 & 74.9 & 67.5 & 24.1 & 12.4 & 38.5 \\
\hline Belgien & 61.6 & 26.8 & 79.3 & 33.8 & 68.2 & 54.9 & 49.0 & 73.2 & 83.6 & 63.5 & 50.9 & 18.3 & 6.3 & 32.9 \\
\hline Dänemark & 77.3 & 67.4 & 86.1 & 58.7 & 81.3 & 73.3 & 62.8 & 81.3 & 87.4 & 78.8 & 62.7 & 17.7 & 12.4 & 23.9 \\
\hline Deutschland & 69.0 & 45.9 & 80.3 & 51.3 & 74.7 & 63.2 & 53.8 & 72.5 & 84.3 & 70.9 & 61.1 & 22.2 & 7.9 & 39.2 \\
\hline Finnland & 70.5 & 46.4 & 83.3 & 55.0 & 72.4 & 68.5 & 58.4 & 75.6 & 85.0 & 70.5 & 63.5 & 11.7 & 8.2 & 15.5 \\
\hline Frankreich & 64.0 & 30.1 & 82.1 & 38.3 & 68.6 & 59.4 & 58.1 & 75.6 & 83.0 & 65.3 & 58.5 & 13.4 & 5.0 & 23.1 \\
\hline Griechenland & 61.5 & 24.2 & 75.7 & 42.1 & 74.9 & 48.1 & 59.5 & 69.7 & 83.3 & 60.9 & 66.6 & 7.8 & 4.1 & 13.6 \\
\hline Irland & 69.0 & 48.8 & 78.8 & 54.1 & 77.4 & 60.3 & 58.7 & 77.3 & 86.5 & 68.2 & 72.9 & 20.3 & 7.6 & 35.6 \\
\hline Island & 85.7 & 74.3 & 89.4 & 84.9 & 89.5 & 81.7 & 83.6 & 88.6 & 92.0 & 84.6 & 84.2 & 15.9 & 8.0 & 25.4 \\
\hline Italien & 58.7 & 24.7 & 73.5 & 33.8 & 70.7 & 46.6 & 52.5 & 74.4 & 80.6 & 58.0 & 65.9 & 15.1 & 5.4 & 29.9 \\
\hline Japan & 70.7 & 41.5 & 80.2 & 66.1 & 81.7 & 59.5 & & 73.1 & 79.8 & .. & .. & 18.9 & 9.2 & 32.6 \\
\hline Kanada & 73.6 & 59.5 & 82.2 & 57.1 & 77.2 & 70.1 & 57.0 & 76.0 & 82.8 & 73.0 & 70.5 & 18.2 & 11.0 & 26.1 \\
\hline Korea & 63.9 & 25.7 & 74.0 & 60.6 & 74.7 & 53.2 & 66.2 & 70.3 & 77.2 & .. & .. & 8.9 & 6.3 & 12.5 \\
\hline Luxemburg & 63.0 & 22.1 & 80.1 & 34.3 & 72.4 & 53.5 & 60.8 & 73.4 & 85.2 & 59.2 & 71.1 & 13.1 & 1.6 & 28.8 \\
\hline Mexiko & 61.1 & 44.2 & 70.3 & 54.7 & 80.9 & 43.6 & 62.8 & 73.1 & 83.3 & .. & .. & 15.1 & 8.1 & 27.6 \\
\hline Neuseeland & 75.4 & 58.7 & 82.2 & 72.0 & 82.1 & 69.0 & 70.6 & 84.5 & 84.6 & .. & .. & 22.0 & 11.2 & 34.7 \\
\hline Niederlande & 74.1 & 65.4 & 83.6 & 50.1 & 80.0 & 68.1 & 60.6 & 79.1 & 86.4 & 77.0 & 62.4 & 36.1 & 16.2 & 60.0 \\
\hline Norwegen & 77.5 & 56.0 & 85.8 & 69.0 & 80.4 & 74.6 & 64.7 & 83.1 & 89.2 & 77.2 & 69.9 & 20.4 & 10.5 & 31.6 \\
\hline Österreich & 71.4 & 55.5 & 84.0 & 38.6 & 78.4 & 64.4 & 55.7 & 75.8 & 85.9 & 72.7 & 65.0 & 17.2 & 5.2 & 31.5 \\
\hline Polen & 57.0 & 25.8 & 74.9 & 29.7 & 63.6 & 50.6 & 53.6 & 65.6 & 83.5 & 57.1 & 36.8 & 10.1 & 6.0 & 15.0 \\
\hline Portugal & 67.8 & 34.9 & 81.0 & 50.9 & 73.9 & 61.9 & 71.7 & 80.2 & 86.4 & 67.3 & 73.1 & 10.0 & 6.3 & 14.3 \\
\hline Schweden & 75.7 & 46.3 & 86.1 & 70.1 & 78.0 & 73.2 & 66.9 & 81.9 & 87.3 & 76.2 & 63.1 & 14.4 & 9.5 & 19.7 \\
\hline Schweiz & 78.6 & 62.6 & 86.1 & 67.2 & 85.6 & 71.6 & 65.3 & 80.1 & 90.2 & 80.3 & 73.7 & 25.4 & 8.7 & 45.6 \\
\hline Slowak. Rep. & 60.7 & 27.6 & 78.0 & 35.7 & 68.4 & 53.0 & 23.5 & 71.9 & 84.9 & 60.7 & 66.0 & 2.6 & 1.2 & 4.4 \\
\hline Spanien & 66.6 & 42.9 & 76.8 & 44.6 & 77.4 & 55.5 & 59.8 & 75.9 & 83.4 & 64.4 & 70.3 & 10.9 & 3.8 & 20.9 \\
\hline Tschech. Rep. & 66.1 & 28.5 & 83.5 & 46.0 & 74.8 & 57.3 & 43.9 & 75.6 & 85.1 & 66.1 & 67.3 & 3.5 & 1.7 & 5.9 \\
\hline Türkei & 45.8 & 30.4 & 54.2 & 29.4 & 67.9 & 23.8 & 49.0 & 62.7 & 75.5 & 45.7 & 48.7 & 8.4 & 4.6 & 19.2 \\
\hline Ungarn & 57.3 & 21.0 & 74.6 & 33.1 & 64.0 & 50.9 & 38.2 & 70.4 & 81.8 & 57.2 & 64.6 & 2.8 & 1.6 & 4.2 \\
\hline Ver. Königreich & 72.3 & 55.9 & 81.3 & 57.4 & 78.4 & 66.3 & 66.3 & 80.7 & 88.1 & 71.8 & 66.3 & 23.3 & 9.9 & 38.6 \\
\hline Ver. Staaten & 71.8 & 53.1 & 79.9 & 61.8 & 77.8 & 65.9 & 58.0 & 73.3 & 82.7 & 69.5 & 70.8 & 12.6 & 7.6 & 17.9 \\
\hline OECD & 68.0 & 43.7 & 79.6 & 51.2 & 75.8 & 60.3 & 58.4 & 75.9 & 84.4 & 68.1 & 65.1 & 15.3 & 7.2 & 25.6 \\
\hline
\end{tabular}

Quelle: OECD (2008), OECD Employment Outlook, OECD, Paris (www.oecd.org/els/employment/outlook); und OECD (2008), International Migration Outlook (www.oecd.org/els/migration/imo). 


\section{Definition und Messung}

Die Arbeitslosenquote ist der Anteil der Personen ohne Beschäftigung, die aktiv Arbeit suchen, an der Bevölkerung im erwerbsfähigen Alter (15-64 Jahre), d.h. an der Zahl der Personen, die eine Beschäftigung haben oder aktiv suchen. Die Daten stammen aus Arbeitskräfteerhebungen der Mitgliedsländer. Gemäß der ILO-Standarddefinition, die diesen Erhebungen zu Grunde gelegt wird, gelten als arbeitslose Personen diejenigen, die in der Berichtswoche nicht mindestens eine Stunde gearbeitet haben, die aber dem Arbeitsmarkt zur Verfügung stehen und in den vier Wochen vor der Erhebung aktive Schritte der Arbeitsuche unternommen haben. Demzufolge werden beispielsweise Personen, die auf Grund physischer Behinderungen nicht arbeiten können oder die nicht aktiv Arbeit suchen, weil sie geringe Hoffnung haben, eine Beschäftigung zu finden, nicht als arbeitslos betrachtet.

Dieser Abschnitt enthält auch Daten über die Inzidenz der Langzeitarbeitslosigkeit unter allen Arbeitslosen. Für die Langzeitarbeitslosen gibt es zwei alternative Definitionen: Personen, die seit mindestens sechs Monaten arbeitslos sind, und Personen mit einer Arbeitslosigkeitsdauer von mindestens zwölf Monaten.

Die durchschnittliche Arbeitslosenquote im OECD-Raum betrug im Jahr 2007 5,7\%. In Island und Norwegen lagen die Arbeitslosenquoten unter 3\%. Über 10\% verblieben sie hingegen in der Slowakischen Republik und der Türkei (Tabelle SS2.3).

Von 2005 bis 2007 sank die durchschnittliche Arbeitslosenquote im OECD-Raum um etwa 1 Prozentpunkt. Die Arbeitslosigkeit hatte zuvor in vier aufeinander folgenden Jahren seit 2001 geringfügig zugenommen (Abbildung SS2.1). Die Entwicklung gestaltete sich jedoch in dieser Zeit in den einzelnen Ländern recht unterschiedlich. Rückgänge wurden in 15 Ländern registriert (insbesondere in der Slowakischen Republik, in Spanien und Polen), und in weiteren 15 Ländern wurde ein Anstieg verzeichnet (dar- unter Deutschland, Portugal und Schweden). Die jüngste Finanzkrise dürfte ebenfalls in vielen OECD-Ländern für steigende Arbeitslosenquoten sorgen.

Bei jungen Menschen, Migranten und Personen mit niedrigerem Bildungsstand ist die Wahrscheinlichkeit der Arbeitslosigkeit höher. Das Altersmuster findet sich durchweg in allen 30 OECD-Ländern. Die durchschnittliche Arbeitslosenquote junger Menschen im OECD-Raum (12\%) liegt wesentlich höher als die der Bevölkerung im Erwerbsalter zwischen 25 und 54 Jahren (5\%) und der älteren Personen (4\%). Die Arbeitslosenquote der Migranten liegt etwas höher als die der im Inland Geborenen (im Durchschnitt 3 Prozentpunkte höher). Dieses Muster gilt nicht für die Türkei, Ungarn und die Vereinigten Staaten, wo die Arbeitslosenquote bei Migranten niedriger liegt. In der Schweiz, Österreich, Belgien, den Niederlanden und den nordischen Ländern ist die Arbeitslosenquote der Migranten hingegen mehr als doppelt so hoch wie die der im Inland geborenen Bevölkerung. Das Bildungsmuster ist in allen Ländern außer Mexiko, Korea und der Türkei zu beobachten, dort liegt die Wahrscheinlichkeit der Arbeitslosigkeit für Menschen mit höherem Bildungsniveau geringfügig höher.

Eine erfreuliche Konvergenz war in vielen Ländern bei den Arbeitslosenquoten von Frauen und Männern festzustellen. Derzeit liegt die Wahrscheinlichkeit der Arbeitslosigkeit bei Frauen nur marginal höher als bei Männern. Die vier Mittelmeerländer - Griechenland, Spanien, Italien und Portugal - wiesen jedoch im Jahr 2007 weiterhin Arbeitslosigkeitslücken von über 3 Prozentpunkten zu Lasten der Frauen auf. Abbildung SS2.2 zeigt, dass die geschlechtsspezifischen Unterschiede bei den Beschäftigungsquoten Anfang der 1990er Jahre tatsächlich in drei Vierteln dieser Länder wesentlich größer waren als 2007. Griechenland bildet hier eine Ausnahme mit einer geringen Verbesserung der geschlechtsspezifischen Ungleichheit.

\section{Anmerkungen zu Abbildungen und Tabellen}

Abbildung SS2.2: Die 1990 am nächsten liegenden Zahlen waren aus 1991 für Island, Mexiko und die Schweiz, aus 1992 für Ungarn und Polen, aus 1993 für die Tschechische Republik, aus 1994 für Österreich und die Slowakische Republik.

Tabelle SS2.3: Anmerkung 1: 2006 nach Bildungsabschluss. Anmerkung 2: 2006 für Kanada, Island und die Vereinigten Staaten. 
SS2.1. Die OECD-weite Arbeitslosenquote sinkt Arbeitslosenquote der 15- bis 64-Jährigen, in Prozent der Erwerbsbevölkerung, 1980-2007

$$
\begin{aligned}
& \text { - Slowak. Rep. - Spanien - - Korea } \\
& \text {..... Mexiko - - - Polen — OECD }
\end{aligned}
$$

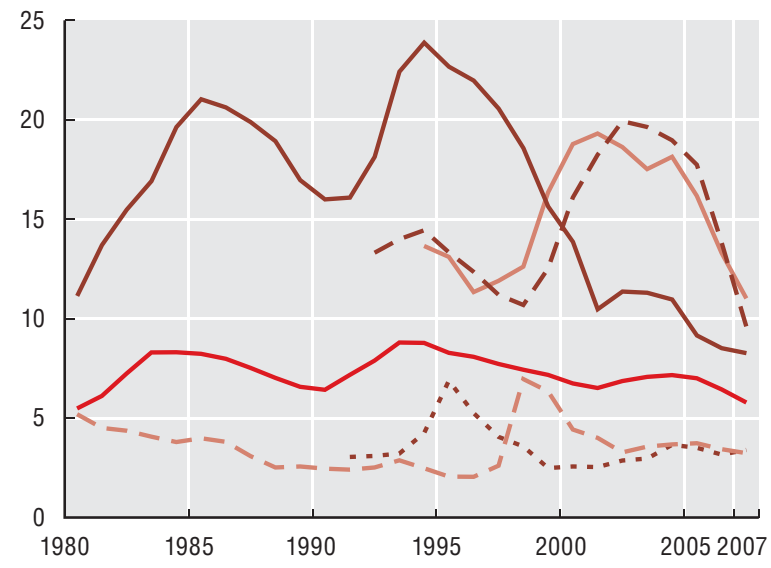

SS2.2. Geschlechtsspezifische Arbeitslosigkeitslücken zu Lasten der Frauen allgemein rückläufig

Prozentuale Unterschiede zwischen Männern und Frauen bei den Arbeitslosenquoten, 1990 oder letztes verfügbares Jahr und 2007

$$
1990 \text { oder letztes verfügbares Jahr } 2007
$$

\begin{tabular}{|c|c|c|c|c|c|c|c|c|c|c|c|c|c|}
\hline \multirow{3}{*}{ 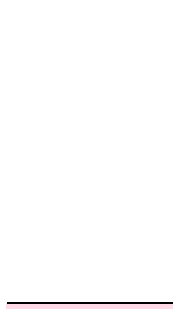 } & \multirow{3}{*}{$\begin{array}{c}\text { Arbeitslosen- } \\
\text { quote für die } \\
\text { Bevölkerung } \\
\text { zwischen } \\
15 \text { und } \\
64 \text { Jahren }\end{array}$} & \multicolumn{10}{|c|}{ Arbeitslosenquote nach: } & \multirow{2}{*}{\multicolumn{2}{|c|}{$\begin{array}{l}\text { Inzidenz der Langzeit- } \\
\text { arbeitslosigkeit } \\
\text { in \% der Gesamt- } \\
\text { arbeitslosigkeit }\end{array}$}} \\
\hline & & \multicolumn{3}{|c|}{ Alter } & \multicolumn{2}{|c|}{ Geschlecht } & \multicolumn{3}{|c|}{ Bildungsabschluss ${ }^{1}$} & \multicolumn{2}{|c|}{ Migrantenstatus $^{2}$} & & \\
\hline & & $15-24$ & $25-54 \mathrm{~J}$. & $55-64 \mathrm{~J}$. & Männer & Frauen & $\begin{array}{l}\text { Ohne- } \\
\text { Sekundar- } \\
\text { stufe-II- } \\
\text { Abschluss }\end{array}$ & $\begin{array}{l}\text { Sekundar- } \\
\text { stufe-II- } \\
\text { Abschluss }\end{array}$ & $\begin{array}{c}\text { Tertiär- } \\
\text { abschluss }\end{array}$ & $\begin{array}{l}\text { Im } \\
\text { Inland } \\
\text { geboren }\end{array}$ & $\begin{array}{l}\operatorname{lm} \\
\text { Ausland } \\
\text { geboren }\end{array}$ & $\begin{array}{c}\text { Mind. } \\
6 \text { Monate }\end{array}$ & $\begin{array}{c}\text { Mind. } \\
12 \text { Monate }\end{array}$ \\
\hline Australien & 4.4 & 9.4 & 3.4 & 2.7 & 4.1 & 4.8 & 6.2 & 3.9 & 2.8 & 4.1 & 4.7 & 27.1 & 15.5 \\
\hline Belgien & 7.7 & 19.2 & 6.8 & 3.8 & 6.7 & 8.8 & 11.7 & 6.9 & 3.9 & 6.4 & 16.4 & 68.1 & 50.0 \\
\hline Dänemark & 3.6 & 7.2 & 2.7 & 4.2 & 3.3 & 4.1 & 7.8 & 4.8 & 3.9 & 3.4 & 8.2 & 29.5 & 18.2 \\
\hline Deutschland & 8.7 & 11.7 & 8.0 & 10.3 & 8.6 & 8.9 & 20.5 & 11.2 & 5.5 & 7.8 & 14.3 & 71.3 & 56.6 \\
\hline Finnland & 6.9 & 15.7 & 5.3 & 6.5 & 6.5 & 7.3 & 12.0 & 8.2 & 4.7 & 6.7 & 14.4 & 37.9 & 23.0 \\
\hline Frankreich & 8.0 & 18.7 & 6.9 & 5.1 & 7.5 & 8.6 & 12.1 & 7.6 & 6.2 & 7.4 & 13.4 & 58.5 & 40.4 \\
\hline Griechenland & 8.2 & 22.0 & 7.6 & 3.4 & 5.0 & 12.8 & 8.4 & 9.7 & 6.9 & 8.4 & 8.7 & 68.2 & 50.3 \\
\hline Irland & 4.6 & 8.7 & 4.1 & 2.6 & 4.9 & 4.3 & 6.4 & 3.2 & 2.1 & 4.4 & 5.9 & 50.1 & 30.3 \\
\hline Island & 2.3 & 7.2 & 1.3 & 0.9 & 2.3 & 2.4 & 3.1 & 2.8 & 1.0 & 2.9 & - & 11.1 & 8.0 \\
\hline Italien & 6.2 & 20.3 & 5.3 & 2.4 & 5.0 & 7.9 & 7.8 & 5.3 & 4.8 & 6.0 & 7.9 & 65.4 & 49.9 \\
\hline Japan & 4.1 & 7.7 & 3.7 & 3.4 & 4.1 & 3.9 & 6.7 & 5.4 & 3.7 & .. & .. & 47.5 & 32.0 \\
\hline Kanada & 6.1 & 11.2 & 5.1 & 5.0 & 6.4 & 5.7 & 9.9 & 6.1 & 4.7 & 6.5 & 7.0 & 14.8 & 7.5 \\
\hline Korea & 3.4 & 8.8 & 3.1 & 2.2 & 3.8 & 2.8 & 2.6 & 3.5 & 2.9 & .. & .. & 11.7 & 0.6 \\
\hline Luxemburg & 3.9 & 14.9 & 3.3 & 0.7 & 4.1 & 3.5 & 5.0 & 3.8 & 3.0 & 3.6 & 4.6 & 54.7 & 33.5 \\
\hline Mexiko & 3.5 & 6.7 & 2.7 & 1.6 & 3.3 & 3.8 & 1.9 & 2.8 & 3.0 & & & 5.4 & 2.7 \\
\hline Neuseeland & 3.7 & 9.7 & 2.5 & 1.4 & 3.4 & 3.9 & 4.2 & 2.4 & 2.4 & 4.8 & 6.0 & 16.7 & 5.7 \\
\hline Niederlande & 3.7 & 7.3 & 2.7 & 4.1 & 3.2 & 4.2 & 5.7 & 3.9 & 2.8 & 3.1 & 7.6 & 59.1 & 41.7 \\
\hline Norwegen & 2.5 & 7.5 & 1.9 & 1.0 & 2.6 & 2.5 & 3.6 & 3.8 & 2.4 & 2.3 & 5.1 & 25.1 & 8.5 \\
\hline Österreich & 4.5 & 8.7 & 3.8 & 3.0 & 4.0 & 5.1 & 7.8 & 3.8 & 2.9 & 3.5 & 9.0 & 44.2 & 26.8 \\
\hline Polen & 9.7 & 21.7 & 8.4 & 6.8 & 9.1 & 10.4 & 27.8 & 17.4 & 6.2 & 9.7 & 9.5 & 64.3 & 45.9 \\
\hline Portugal & 8.5 & 16.6 & 7.8 & 6.5 & 7.0 & 10.1 & 6.4 & 5.6 & 4.4 & 8.4 & 9.6 & 67.6 & 47.3 \\
\hline Schweden & 6.2 & 18.9 & 4.4 & 3.9 & 5.9 & 6.4 & 6.5 & 5.8 & 4.3 & 5.3 & 12.1 & 27.3 & 13.0 \\
\hline Schweiz & 3.7 & 7.1 & 3.1 & 3.1 & 3.0 & 4.6 & 7.2 & 3.7 & 2.8 & 2.6 & 7.1 & 56.6 & 40.8 \\
\hline Slowak. Rep. & 11.0 & 20.1 & 10.1 & 8.1 & 9.8 & 12.6 & 47.7 & 14.6 & 4.8 & 11.2 & - & 82.3 & 70.8 \\
\hline Spanien & 8.3 & 18.2 & 7.2 & 5.9 & 6.4 & 10.9 & 11.0 & 9.5 & 7.3 & 7.9 & 10.3 & 42.6 & 27.6 \\
\hline Tschech. Rep. & 5.4 & 10.7 & 4.9 & 4.6 & 4.3 & 6.8 & 23.0 & 6.4 & 2.0 & 5.3 & 9.0 & 71.6 & 53.4 \\
\hline Türkei & 10.1 & 19.6 & 8.3 & 3.8 & 10.0 & 10.5 & 8.1 & 10.1 & 8.2 & 8.7 & 8.3 & 46.3 & 30.4 \\
\hline Ungarn & 7.4 & 18.0 & 6.8 & 4.2 & 7.2 & 7.7 & 10.8 & 5.0 & 1.9 & 7.5 & 4.3 & 64.0 & 47.5 \\
\hline Ver. Königreich & 5.3 & 14.4 & 3.7 & 3.3 & 5.6 & 4.9 & 6.6 & 3.7 & 2.2 & 4.9 & 7.6 & 41.5 & 24.7 \\
\hline Ver. Staaten & 4.7 & 10.5 & 3.7 & 3.1 & 4.8 & 4.6 & 10.5 & 5.6 & 3.3 & 5.3 & 4.4 & 17.6 & 10.0 \\
\hline OECD & 5.7 & 11.9 & 4.9 & 4.0 & 5.5 & 6.0 & 10.3 & 6.2 & 3.9 & 5.7 & 8.6 & 42.3 & 29.1 \\
\hline
\end{tabular}

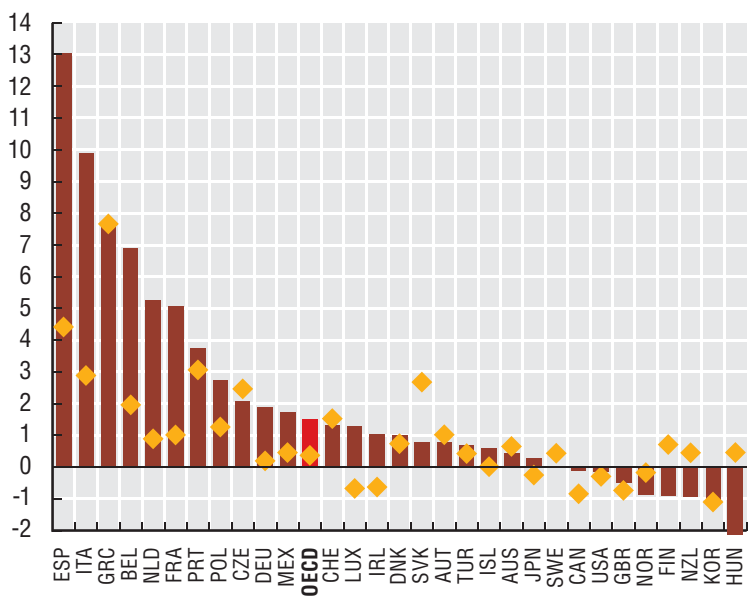

SS2.3. Arbeitslosigkeitsindikatoren, 2007

Quelle: OECD (2008), Employment Outlook, OECD, Paris (www.oecd.org/els/employment/outlook); und OECD (2008), International Migration Outlook, OECD, Paris (www.oecd.org/els/migration/imo). 


\section{Definition und Messung}

Die Unterbringung der 0- bis 2-Jährigen in Kinderbetreuungsstrukturen beinhaltet die Teilnahme an formalen Betreuungsformen wie Krippen, registrierte Kinderbetreuer, aber auch Betreuung durch eine Person, die kein Familienmitglied ist. Die Betreuungsquoten für Kinder von drei bis fünf Jahren beziehen sich auf die Teilnahme an formalen Vorschulstrukturen und in manchen Ländern hinsichtlich der 4- und 5-Jährigen auf die Einschreibung in Grundschulen.

Die Daten zur Teilnahme an Kinderbetreuungsstrukturen unter den 0- bis 2-Jährigen stammen aus verschiedenen Quellen, wodurch die Vergleichbarkeit eingeschränkt wird. Zu den Quellen gehören die OECD Babies and Bosses reviews, die OECD Education database, die Eurydice-Datenbank, NOSOSCOBerichte und nationale Statistikämter. Die Teilnahme der 3- bis 5-Jährigen wurde auf der Basis der OECD Education database dargestellt. Diese Informationen beziehen sich auf die tatsächliche Anzahl der Schüler, die an formalen Vorschulprogrammen teilnehmen, woraus ein Prozentsatz errechnet wird, indem die Bevölkerungsdaten als Nenner verwendet werden.

Im OECD-Raum ist im Durchschnitt jedes fünfte Kind unter drei Jahren in formalen Kinderbetreuungsstrukturen untergebracht (Abbildung SS3.1). Die Quote für Kinder dieser Altersgruppe liegt in Deutschland, Griechenland, Italien, Mexiko, Österreich, Polen, der Tschechischen Republik und Ungarn bei unter $10 \%$, in den nordischen Ländern hingegen bei mindestens $40 \%$. Besonders hoch liegen die Teilnahmequoten in Dänemark (62\%) und Island (59\%). Diese Länderunterschiede spiegeln Unterschiede bei der Verfügbarkeit von öffentlichen Betreuungseinrichtungen, bei den Elternzeitsystemen, bei weiteren Anreizen zur Arbeitsaufnahme der Frauen sowie bei der Kultur und den Familienstrukturen wider. Die Teilnahmezahlen geben keine Auskunft zur Dauer der Betreuung. Auch die in formalen Betreuungseinrichtungen verbrachte Zeit variiert wahrscheinlich stark von Land zu Land. Diese Unterschiede können von Bedeutung sein, denn die Intensität der Betreuung spielt eine wichtige Rolle sowohl bei den Entwicklungsergebnissen der Kinder (die Zeitspanne kann zu lang oder zu kurz sein) als auch bei der Teilnahme der Mütter am Arbeitsmarkt.

Ländervergleiche sind jedoch auf Grund von Datenproblemen schwierig. Möglicherweise wird die formale Kinderbetreuung in den Ländern zu niedrig ausgewiesen, in denen ein beträchtlicher Anteil der Betreuung privat in Zentren oder daheim durch familienfremde Betreuer geleistet wird
(z.B. in Australien, Irland, Kanada, Mexiko und den Vereinigten Staaten) oder in denen die Bildung und Betreuung der Vorschulkinder von nachgeordneten Gebietskörperschaften wahrgenommen und finanziert wird (z.B. in Kanada, Mexiko, der Schweiz und den Vereinigten Staaten). Diese Untererfassung kann zu einer Unterschätzung der Teilnahmequoten führen. Des Weiteren sind die Kinder in manchen Ländern u.U. in mehreren Teilzeitprogrammen gleichzeitig eingeschrieben, was zu Doppelzählungsproblemen und folglich zu einer Überschätzung der Teilnahmequoten führt.

Im OECD-Durchschnitt sind über $70 \%$ der 3- bis 5-Jährigen in Vorschulen eingeschrieben (Abbildung SS3.2). Der Anteil liegt wesentlich höher als die Teilnahme jüngerer Kinder an Betreuungssystemen. In der Hälfte der OECDLänder waren im Jahr 2006 mindestens 80\% der Kinder dieser Altersgruppe in Vorschulprogrammen eingeschrieben. Quasi universell sind die Teilnahmequoten in Frankreich, Belgien, Italien und Spanien. Hingegen sind in der Türkei, Polen und Korea wenige Kinder beteiligt, was eine erhebliche Nutzung der familiären Betreuung widerspiegelt.

Der Anteil der Vorschulkinder ist gestiegen. Von 20002005 stiegen die durchschnittlichen Vorschulteilnahmequoten von Kindern zwischen drei und fünf Jahren um 4 Prozentpunkte, von $69 \%$ auf $73 \%$. Zu den Ländern mit hohen Zuwächsen gehören Deutschland, Mexiko und Norwegen.

Die nordischen Länder, Frankreich und Belgien geben den höchsten Anteil ihres Nettonationaleinkommens für Kinderbetreuung aus (Abbildung SS3.3). Am unteren Ende der Skala befinden sich die Schweiz, Korea, Kanada und Griechenland. Die Länderdifferenzen bei den Ausgaben ergeben sich aus den unterschiedlichen Anteilen von Kleinkindern an der Gesamtbevölkerung, aus den Teilnahmequoten und aus den Ausgaben pro Kind.

\section{Weitere Informationen}

OECD (verschiedene Ausgaben), Babies and Bosses, Paris (www.oecd.org/els/social/family).

OECD (2008), Bildung auf einen Blick 2008: OECD-Indikatoren, W. Bertelsmann Verlag.

\section{Anmerkungen zu den Abbildungen}

Abbildung SS3.1: Die Länder sind in absteigender Reihenfolge der Teilnahmequoten von 3- bis 5-Jährigen geordnet. Für die Schweiz und die Türkei liegen keine Daten vor, 2000 für Irland und Italien, 2001 für Deutschland und Polen, 2002 für Frankreich, 2003 für Griechenland, Island, Luxemburg, Mexiko, Norwegen und die Slowakische Republik, 2005 für Australien, Dänemark, Korea und die Vereinigten Staaten, 2004 für andere Länder. 
SS3.1. Teilnahmequoten an formalen Kinderbetreuungsstrukturen je nach Land sehr unterschiedlich

Durchschnittliche Teilnahmequote der Kinder unter 3 Jahren an formaler Betreuung, 2005 oder letztes verfügbares Jahr, in Prozent

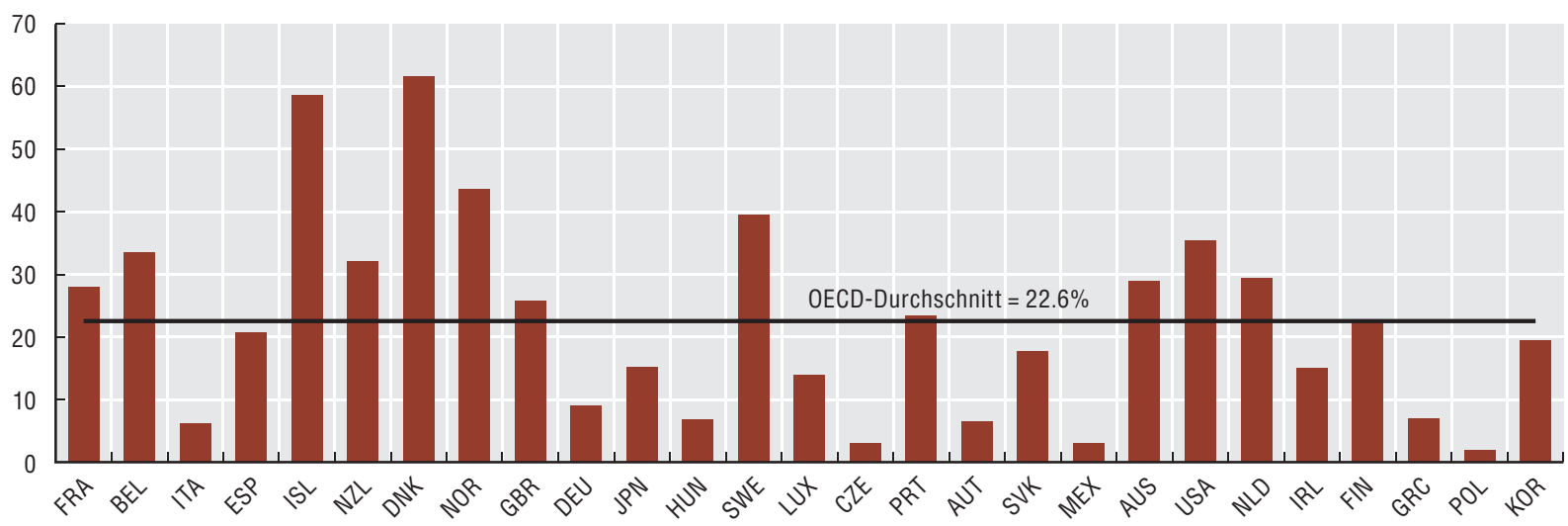

SS3.2. Die Mehrzahl der Vorschulkinder zwischen 3 und 5 Jahren ist in Kinderbetreuung

Durchschnittliche Teilnahmequote der Kinder zwischen 3 und 5 Jahren an Vorschulbildungsprogrammen, in Prozent, 2000 und 2005

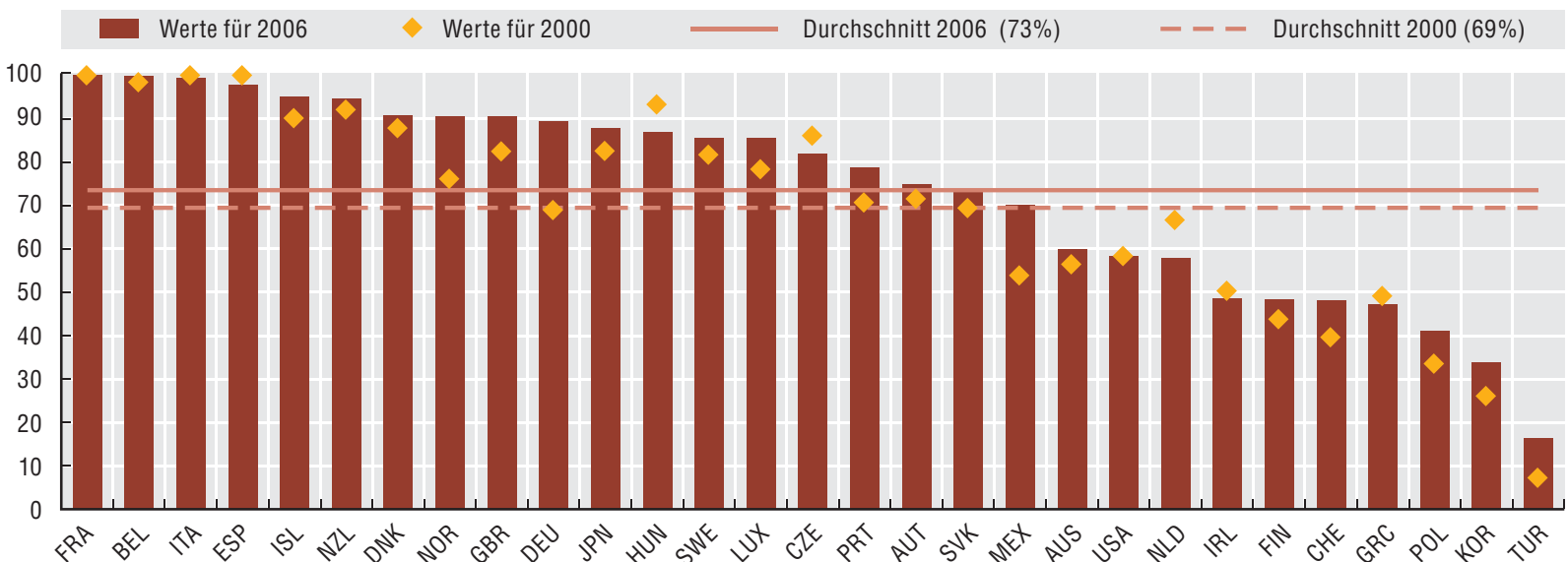

SS3.3. Die nordischen Länder haben wesentlich höhere Ausgaben für Kinderbetreuungsprogramme Öffentliche Ausgaben für Kinderbetreuung, einschließlich Vorschule, in Prozent des NNE, 2005

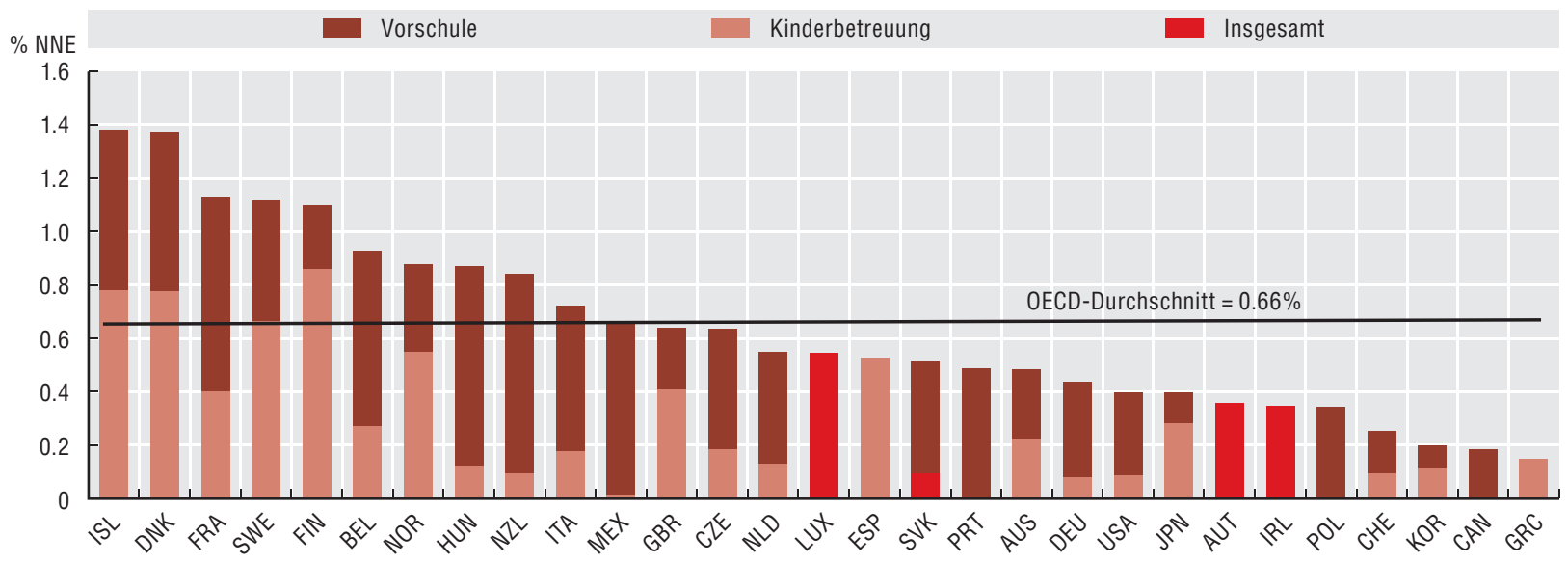




\section{Definition und Messung}

Die Schülerleistungen lassen sich anhand der Ergebnisse der Internationalen Schulleistungsstudie der OECD (PISA) beurteilen. PISA ist das bisher umfassendste und weitreichendste internationale Projekt zur Messung der Kenntnisse und Fähigkeiten von Schülerinnen und Schülern gegen Ende ihrer Pflichtschulzeit. In der letzten Erhebungsrunde wurden 15jährige Schülerinnen und Schüler im Jahr 2006 in allen OECD-Ländern in Lesekompetenz, Mathematik und Naturwissenschaften getestet (die Vereinigten Staaten sind im Lesekompetenztest nicht berücksichtigt). Bei PISA werden standardisierte Tests unter unabhängiger Aufsicht durchgeführt, um die Kompetenzen der Schüler zu evaluieren. Die PISA-Tests sind nicht an bestimmte nationale Lehrpläne gekoppelt. Vielmehr wenden die Schülerinnen und Schüler ihr Wissen auf Situationen an, denen sie in der realen Welt begegnen könnten, wie die Planung einer Reiseroute, die Interpretation der Gebrauchsanleitung für ein elektrisches Gerät oder das Auffinden von Informationen in einer grafischen Darstellung. Für jedes Fach, das zum ersten Mal zu einem Hauptbereich in PISA wird, liegt die mittlere Punktzahl bei 500 . Danach ergibt sich die Leistung der OECD-Länder aus dem OECD-Durchschnitt.

Im Bereich Lesekompetenz sind Ergebnisvergleiche zwischen PISA 2006 und PISA 2000 möglich, was den längsten Betrachtungszeitraum für Zeittrends darstellt (leider sind die Ergebnisse in Naturwissenschaften und Mathematik nicht über diesen längeren Zeitraum vergleichbar).

Neben den mittleren Punktzahlen, die die Schüler in jedem Land in den drei Grundbildungsbereichen erzielten, wird außerdem eine Messgröße der Ungleichheit bei den Testergebnissen innerhalb der Länder verwendet: der Variationskoeffizient (definiert als die landesspezifische Standardabweichung geteilt durch die mittlere Punktzahl).

Schwache Schülerleistungen bei 15-Jährigen können sich dauerhaft auf das Leben der jungen Menschen auswirken. Schwache Lernergebnisse führen zu einer höheren Wahrscheinlichkeit des Schulabbruchs, zu schlechteren längerfristigen Karriereaussichten und Beziehungen sowie einer größeren Wahrscheinlichkeit langfristiger Abhängigkeit von öffentlichen Sozialleistungen. Deshalb ist es in allen OECD-Ländern eine Politikpriorität, dafür Sorge zu tragen, dass Kinder eine gute Bildung erhalten. Sämtliche Länder geben große, wenngleich unterschiedliche Summen an öffentlichen Geldern aus, um sicherzustellen, dass Kinder und Jugendliche gebildet werden. Die Schülerleistungen der PISA-Tests deuten bis zum Alter von 15 Jahren auf einen kumulativen Effekt der Bildungsinputs der Familie, der Schulen, der Gleichaltrigen und der Gemeinschaft hin. Die Testergebnisse sind zwar nicht der einzige
Indikator einer erfolgreichen Schulbildung (die Sozialisierung ist zum Beispiel ganz eindeutig ein wichtiges Ziel), sie werden jedoch fast einvernehmlich als eine sehr wichtige Messgröße betrachtet.

Es gibt erhebliche Länderunterschiede bei den Schülerleistungen gegen Ende der Pflichtschulzeit (Abbildung SS4.1). Die Unterschiede zwischen dem bestplatzierten Land (Korea für Lesekompetenz, Finnland für Mathematik und Naturwissenschaften) und dem am unteren Ende der Skala angesiedelten (Mexiko) betragen über 140 Punkte, was fast eineinhalb Standardabweichungen entspricht. Abbildung SS4.1 zeugt ebenfalls von einer starken Tendenz der Länder, die gute Ergebnisse bei der Lesekompetenz erzielen, bei den Naturwissenschaften und der Mathematik ebenfalls gut abzuschneiden. Die Korrelation zwischen den Ergebnissen der Länder bei der Lesekompetenz sowie in Mathematik und den Naturwissenschaften liegt durchweg über 0,87 .

Der Geschlechterunterschied zu Gunsten der Mädchen bei der Lesekompetenz hat sich mit der Zeit vergrößert. Die Geschlechterunterschiede bei der Lesekompetenz haben sich im Zeitraum 2000-2006 in fast allen OECD-Ländern vergrößert (Abbildung SS4.2). In den meisten Ländern ist dieser Anstieg zwar in statistischer Hinsicht nicht erheblich, doch die Tatsache, dass dieser Trend bis auf zwei Ausnahmen in allen Ländern festgestellt wird, deutet darauf hin, dass sich möglicherweise systematische Veränderungen zu Gunsten der Mädchen abzeichnen. Die Ergebnisse von 2006 bestätigen außerdem, entgegen den Resultaten bei der Lesekompetenz, dass Jungen in Mathematik bessere Leistungen erzielen als Mädchen. Bei den Naturwissenschaften ist das Bild uneinheitlich. Leider ist ein Vergleich der Geschlechterunterschiede bei Mathematik und Naturwissenschaften über den gleichen Zeitraum nicht möglich.

Länder, in denen die durchschnittlichen Ergebnisse bei der Lesekompetenz von 2000-2006 gewachsen sind, haben außerdem die Ungleichheit bei den Testergebnissen verringert (Abbildung SS4.3). Die Korrelation zwischen der Veränderung der Lesekompetenzergebnisse und der Veränderung der Ungleichheit bei der Lesekompetenz (gemessen am dazugehörigen Veränderungskoeffizienten) liegt bei -0,52. Frühere Ausgaben von Gesellschaft auf einen Blick hatten gezeigt, dass Länder mit hohen durchschnittlichen PISA-Leistungen auch eine niedrige Ungleichheit bei den Testergebnissen aufwiesen, und umgekehrt.

\section{Weitere Informationen}

OECD (2003), Literacy Skills for the World of Tomorrow: Further Results from PISA 2000, Paris.

OECD (2007), PISA 2006: Naturwissenschaftliche Kompetenzen für die Welt von morgen, W. Bertelsmann Verlag (www.pisa.oecd.org)

\section{Anmerkungen zu den Abbildungen}

Abbildung SS4.1: Die Länder sind in absteigender Reihenfolge nach den Schülerleistungen in Mathematik geordnet. 
SS4.1. Große Unterschiede bei den Schülerleistungen in den OECD-Ländern

Mittelwerte auf den Skalen Mathematik, Lesekompetenz und Naturwissenschaften, PISA 2006

Mathematik

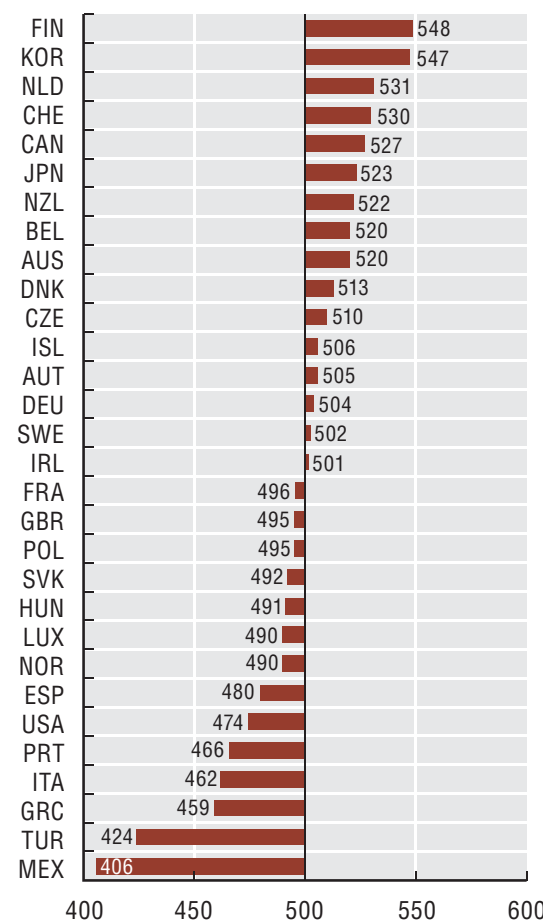

Lesekompetenz

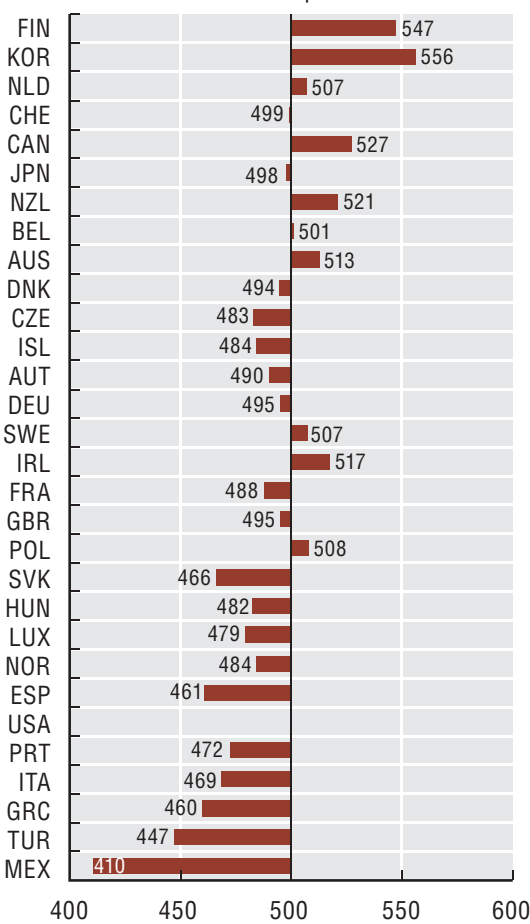

Naturwissenschaften

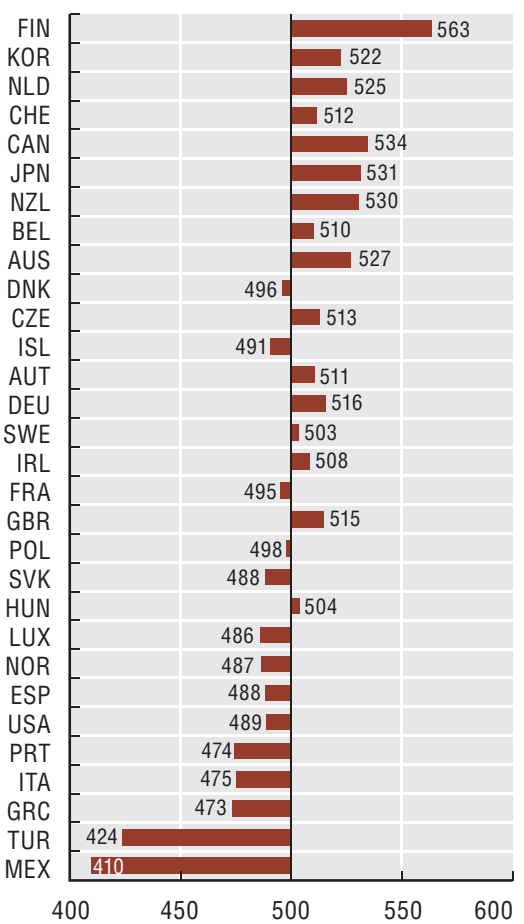

SS4.2. Die Geschlechterunterschiede zu Gunsten der Mädchen bei der Lesekompetenz nehmen zu

Veränderungen der Geschlechterunterschiede bei der Lesekompetenz (weibliche Ergebnisse minus männliche) zwischen 2000 und 2006,

$$
\text { in Punkten }
$$

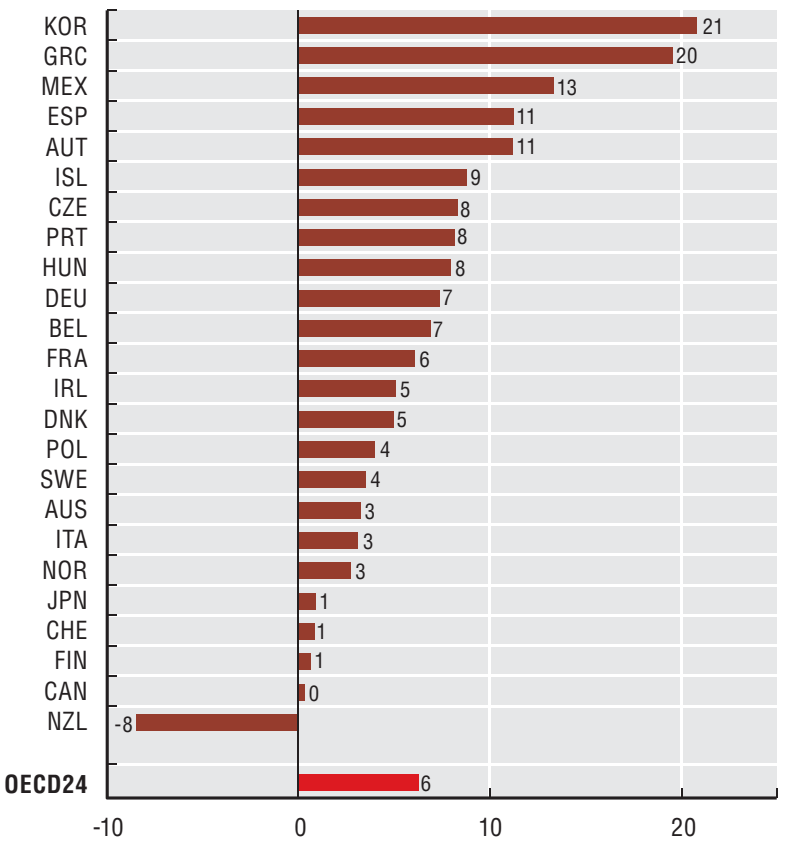

Quelle: OECD (2007), PISA 2006: Naturwissenschaftliche Kompetenzen für die Welt von morgen, W. Bertelsmann Verlag (www.pisa.oecd.org).
SS4.3. Länder mit steigenden durchschnittlichen Ergebnissen bei der Lesekompetenz reduzieren die Ungleichheiten bei den Lernergebnissen

Veränderungen der durchschnittlichen Ergebnisse bei der Lesekompetenz und Veränderungen des Variationskoeffizienten der Lesekompetenz

Veränderung (2006-2000) der Lesekompetenz

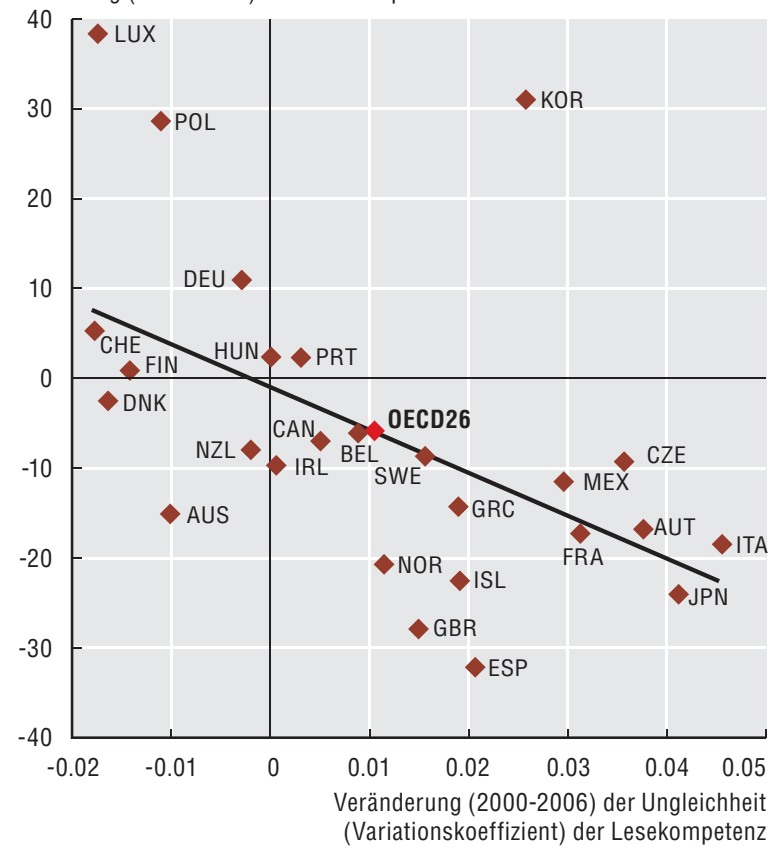

StatLink Aाst http://dx.doi.org/10.1787/550180640382 


\section{Definition und Messung}

Dieser Indikator erfasst die Jugendlichen im Alter von 15-19 Jahren, die sich weder in Beschäftigung noch in Bildung oder Ausbildung befinden, als Anteil an der restlichen Bevölkerung derselben Altersgruppe. Die einzige Ausnahme zur Kohorte der 15- bis 19-Jährigen bildet Japan, wo die Jugendlichen im Alter von 15-24 Jahren berücksichtigt wurden. Unter Bildung fällt sowohl der Teilzeit- als auch der Vollzeitbesuch einer Bildungseinrichtung, informelle und sehr kurzfristige Bildungsangebote sind hingegen ausgeschlossen. Die Daten stammen aus Arbeitskräfteerhebungen und beziehen sich üblicherweise auf einen Zeitraum von vier Wochen vor der Erhebung. Bedingt durch Stichprobenfehler ist eine gewisse zeitliche Datenvolatilität möglich. Die Quoten beziehen sich auf die Jahre 1998-2006. Die Daten stammen aus dem Bericht Bildung auf einen Blick 2008: OECD-Indikatoren.

\section{Nur eine Minderheit von Jugendlichen befindet sich} weder in Beschäftigung noch in Bildung oder Ausbildung. Im Durchschnitt waren im Jahr 2006 im OECD-Raum etwa $6 \%$ der Jugendlichen nicht in Beschäftigung, Bildung oder Ausbildung. Dieser Durchschnitt kaschiert erhebliche Unterschiede zwischen den einzelnen Ländern. Die Quoten liegen in Italien, Neuseeland, Spanien und dem Vereinigten Königreich fast doppelt so hoch wie im OECD-Durchschnitt. Etwa halb so hoch liegen sie dagegen in Norwegen und Polen.

Die Anteile der weder an Beschäftigung noch Bildung oder Ausbildung teilnehmenden Jugendlichen sind in allen OECD-Ländern rückläufig. Bei den männlichen Jugendlichen sanken die Quoten von etwa 8\% im Jahr 1998 auf etwas über $6 \%$ im Jahr 2006, bei den jungen Frauen war der Rückgang vergleichbar. Allerdings fand der Großteil dieser Entwicklung zwischen 1998 und 2002 statt. Danach haben sich die durchschnittlichen Quoten in den OECDLändern weitgehend stabilisiert. Die wichtigsten Motoren des Rückgangs waren die sinkenden Quoten in der Slowakischen Republik (15 Prozentpunkte weniger bei den jungen Männern und 8 Prozentpunkte weniger bei den jungen Frauen zwischen 1998 und 2000) und Ungarn (6 Prozentpunkte weniger sowohl bei jungen Männern als auch bei jungen Frauen). $\mathrm{Zu}$ den Ländern, in denen der Anteil der weder an Beschäftigung noch Bildung oder Ausbildung teilnehmenden Jugendlichen dem OECD-weiten
Trend zuwiderlief und im Beobachtungszeitraum gestiegen ist, gehören Dänemark, Frankreich, Japan, die Schweiz und das Vereinigte Königreich.

Die Anteile der weder an Beschäftigung noch Bildung oder Ausbildung teilnehmenden Jugendlichen konvergieren im gesamten OECD-Raum. Die Anteile der jungen Männer sind insgesamt weniger breit gestreut als die der jungen Frauen.

Die Mehrzahl der weder an Beschäftigung noch Bildung oder Ausbildung teilnehmenden Jugendlichen ist nicht auf Arbeitsuche (Abbildung SS5.3). Lediglich in der Slowakischen Republik und der Tschechischen Republik, in Frankreich, Polen und Österreich sucht mehr als die Hälfte der betroffenen Jugendlichen aktiv eine Beschäftigung. Am entgegengesetzten Ende des Spektrums sind in Mexiko und der Türkei weniger als 15\% der Jugendlichen, die nicht in Beschäftigung, Bildung oder Ausbildung sind, in der Lage und bereit zu arbeiten.

Die weder an Beschäftigung noch Bildung oder Ausbildung teilnehmenden jungen Männer suchen mit höherer Wahrscheinlichkeit eine Arbeit als die inaktiven jungen Frauen. Nur in drei Ländern - Polen, Österreich und Dänemark - gibt es mehr arbeitsuchende junge Frauen als arbeitsuchende junge Männer. Eine Erklärung für die geringere Wahrscheinlichkeit der Arbeitsuche bei Frauen ist, dass sie eher für die Betreuung eines Kindes sorgen. Polen, Österreich und Dänemark, wo die jungen Frauen mit höherer Wahrscheinlichkeit Arbeit suchen, sind Länder mit relativ niedrigen Teenager-Geburtenraten. Dort wo die geschlechtsspezifischen Unterschiede bei der Arbeitsuche am deutlichsten sind, wie in Mexiko und der Türkei, liegen die Teenager-Geburtenraten entsprechend hoch.

\section{Anmerkungen zu den Abbildungen und Tabellen}

Tabellen SS5.1 und SS5.2: In die Vergleiche wurden nur Länder mit aktualisierten Ergebnissen für das Jahr 2006 einbezogen. Die japanischen Daten beziehen sich auf Jugendliche im Alter von 15-24 Jahren. Es fehlen Island, Mexiko und die Türkei.

Abbildung SS5.3: Die japanischen Daten beziehen sich auf Jugendliche im Alter von 15-24 Jahren. Es fehlen Island, Luxemburg und Norwegen. Der Begriff „für Beschäftigung verfügbare inaktive Jugendliche" bezieht sich auf die, die aktiv Arbeit suchen oder annehmen können. Die Daten für Männer in der Slowakischen Republik und für Frauen in Finnland, Schweden und Griechenland sind Schätzungen auf der Basis der gemeldeten Daten für Frauen in der Slowakischen Republik und Männer in Finnland, Schweden und Griechenland sowie der gemeldeten Gesamtergebnisse. 


\section{Nicht in Beschäftigung, Bildung oder Ausbildung}

SS5.1. Anzahl der nicht in Beschäftigung, Bildung oder Ausbildung befindlichen jungen Männer im OECD-Raum relativ stabil

Junge Männer, in Bildung sowie nicht in Bildung, in Prozent, 1998-2006

\begin{tabular}{lrrrrr}
\hline & 1998 & 2000 & 2002 & 2004 & 2006 \\
\hline Australien & 9.0 & 6.4 & 6.9 & 7.6 & 6.7 \\
Belgien & 10.8 & 6.7 & 7.3 & 5.8 & 7.5 \\
Dänemark &.. & 1.9 & 2.4 & 1.9 & 4.8 \\
Deutschland &.. & 5.2 & 4.3 & 3.5 & 4.1 \\
Finnland &.. &.. &.. & 5.4 & 4.1 \\
\hline Frankreich & 3.5 & 3.4 & 3.7 & 5.7 & 6.7 \\
Griechenland & 6.9 & 6.9 & 5.2 & 8.7 & 7.9 \\
\hline Irland &.. & 4.5 & 5.2 & 5.2 & 5.3 \\
Italien & 14.5 & 12.2 & 10.8 & 11.1 & 12.2 \\
Japan & 5.5 & 7.3 & 8.3 & 8.1 & 7.5 \\
Kanada & 9.1 & 8.7 & 8.7 & 9.8 & 8.0 \\
Luxemburg & 6.4 &.. &.. &.. & 4.4 \\
Neuseeland &.. &.. &.. &.. & 9.9 \\
Niederlande & 2.6 & 3.8 & 4.0 & 3.5 & 3.3 \\
Norwegen &.. &.. &.. &.. & 3.5 \\
Österreich &.. &.. & 8.1 & 7.2 & 7.1 \\
Polen & 4.7 & 5.0 & 3.5 & 3.0 & 3.8 \\
Portugal & 6.9 & 6.2 & 7.7 & 9.0 & 7.8 \\
Schweden & 6.4 & 4.7 & 5.9 & 6.4 & 6.2 \\
\hline Schweiz & 4.0 & 7.3 & 5.8 & 7.6 & 7.7 \\
\hline Slowak. Rep. & 21.7 & 27.8 & 17.7 & 8.6 & 6.5 \\
Spanien & 10.1 & 7.7 & 6.9 & 7.3 & 9.6 \\
Tschech. Rep. & 6.7 & 7.3 & 5.8 & 5.0 & 4.7 \\
\hline Ungarn & 12.4 & 8.6 & 8.3 & 6.6 & 6.4 \\
Ver. Königreich &.. & 8.2 & 8.2 & 9.0 & 11.5 \\
Ver. Staaten & 6.5 & 6.8 & 6.4 & 6.5 & 6.0 \\
\hline & & & & & \\
\hline
\end{tabular}

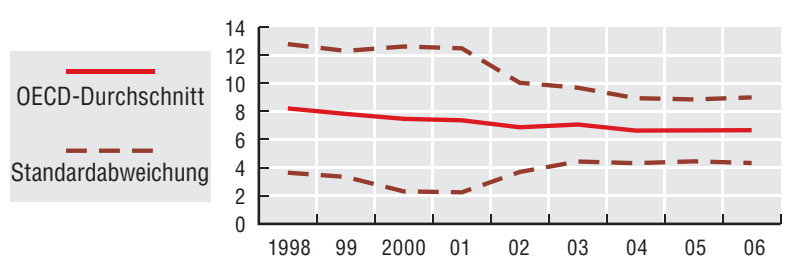

SS5.2. Abweichungen bei Frauen, die nicht in Beschäftigung, Bildung oder Ausbildung sind, im OECD-Raum durchweg größer als bei Männern Junge Frauen, in Bildung sowie nicht in Bildung, in Prozent, 1998-2006

\begin{tabular}{lrrrrr}
\hline & 1998 & 2000 & 2002 & 2004 & 2006 \\
\hline Australien & 8.7 & 7.3 & 7.2 & 7.4 & 7.4 \\
Belgien & 10.8 & 6.3 & 6.4 & 3.9 & 6.7 \\
Dänemark & 2.1 & 3.6 & 2.4 & 2.3 & 4.1 \\
Deutschland &.. & 6.3 & 5.1 & 3.7 & 4.3 \\
Finnland &.. &.. &.. & 6.4 & 3.0 \\
Frankreich & 2.6 & 3.2 & 3.2 & 4.5 & 6.2 \\
Griechenland & 12.7 & 11.2 & 7.5 & 11.3 & 9.8 \\
Irland &.. & 4.3 & 4.5 & 4.6 & 4.7 \\
Italien & 15.9 & 14.1 & 10.3 & 10.9 & 11.4 \\
Japan & 9.8 & 10.3 & 10.6 & 10.4 & 10.5 \\
Kanada & 7.9 & 7.7 & 7.2 & 7.6 & 6.7
\end{tabular}

\begin{tabular}{lrrrrr} 
Neuseeland &.. &.. &.. &.. & 12.7 \\
Niederlande & 2.9 & 3.6 & 3.7 & 3.4 & 2.6 \\
\hline Norwegen &.. &.. &.. &.. & 3.4 \\
Österreich &.. &.. & 4.4 & 7.5 & 6.0 \\
Polen & 4.9 & 4.0 & 2.6 & 2.1 & 3.7 \\
\hline Portugal & 9.7 & 9.2 & 6.8 & 10.6 & 7.7 \\
Schweden & 2.9 & 2.4 & 3.3 & 3.2 & 4.3 \\
\hline Schweiz & 5.7 & 8.5 & 5.8 & 6.8 & 7.5 \\
Slowak. Rep. & 14.9 & 24.7 & 13.5 & 7.1 & 6.8 \\
\hline Spanien & 9.6 & 8.2 & 7.5 & 7.9 & 10.6 \\
Tschech. Rep. & 7.7 & 8.5 & 6.3 & 6.4 & 4.3 \\
\hline Ungarn & 11.1 & 8.6 & 7.8 & 5.8 & 5.6 \\
\hline Ver. Königreich &.. & 7.9 & 8.9 & 9.0 & 10.3 \\
\hline Ver. Staaten & 8.2 & 7.3 & 7.5 & 7.3 & 6.7 \\
\hline
\end{tabular}

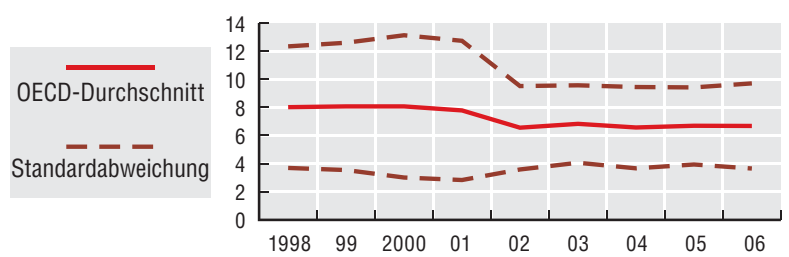

SS5.3. Die Mehrzahl der nicht in Beschäftigung, Bildung oder Ausbildung befindlichen jungen Frauen steht in der Regel nicht für Beschäftigung zur Verfügung

Für Arbeit zur Verfügung stehend oder auf Arbeitsuche, nach Geschlecht, in Prozent, 2006

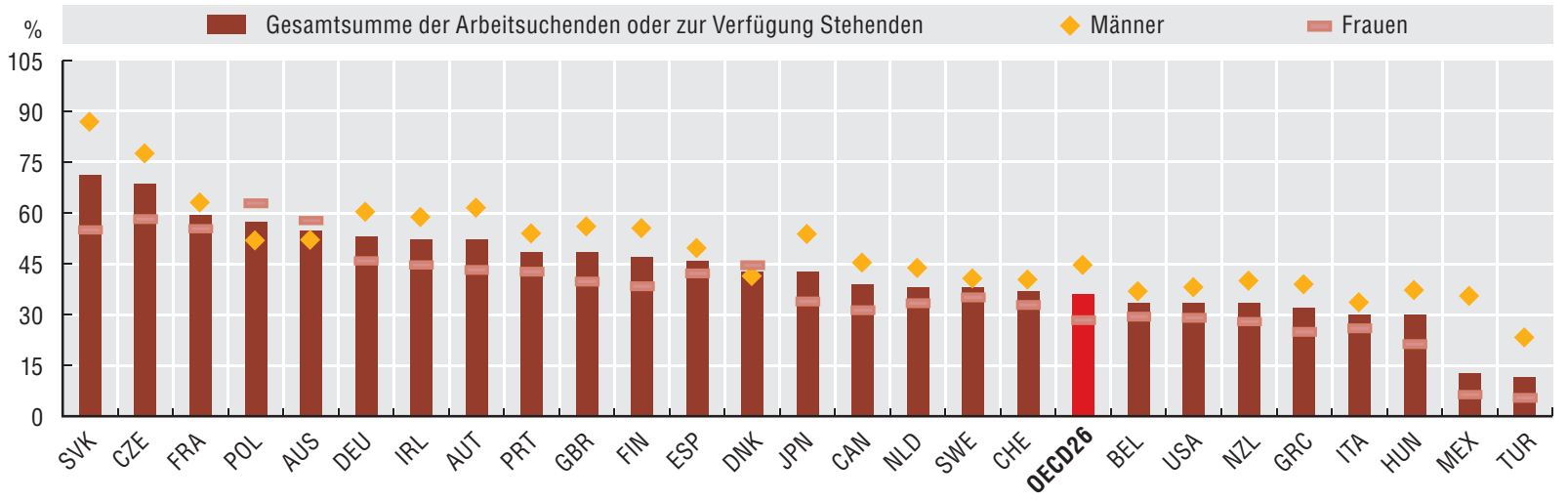

Quelle: OECD (2008), Bildung auf einen Blick, W. Bertelsmann Verlag (www.oecd.org/edu/EAG2008) 


\section{Definition und Messung}

Der Eintritt in den Ruhestand wird mit der Beendigung der Erwerbstätigkeit und dem Erhalt einer Rente in Verbindung gebracht. Das tatsächliche Renteneintrittsalter ist ohne international vergleichbare längere Zeitreihen nur schwer direkt zu messen, deshalb müssen sich internationale Vergleiche auf indirekte Messungen aus Querschnittsdaten stützen. Indirekte Messungen betrachten Personen ab einem bestimmten Alter als „im Ruhestand“, wenn sie zum Zeitpunkt der Erhebung nicht zur Erwerbsbevölkerung gehören (durchschnittliches Alter bei Austritt aus dem Erwerbsleben). Die Nettozahlen der in den Ruhestand tretenden Personen ergeben sich aus Berechnungen anhand von Veränderungen im Zeitverlauf des Anteils der älteren Bevölkerung, die nicht erwerbstätig ist. Diese indirekte Messgröße wird in derzeitigen OECD-Studien über ältere Arbeitnehmer verwendet. Gemessen wird das durchschnittliche tatsächliche Renteneintrittsalter. Das gesetzliche Renteneintrittsalter ist ebenfalls schwierig $\mathrm{zu}$ bestimmen, insbesondere wenn der Renteneintritt an festgelegte Beitragsjahre geknüpft ist. Für nähere Informationen vgl. OECD (2007).

In den OECD-Ländern liegt das gesetzliche Renteneintrittsalter sowohl für Männer als auch für Frauen am häufigsten bei 65 Jahren. Höhere und niedrigere Mindestalter gibt es in mehreren Ländern (Abbildung SS6.1). Obwohl die Lebenserwartung der Frauen höher ist und Männer im Durchschnitt höhere Beiträge zahlen, ist im OECD-Durchschnitt das gesetzliche Renteneintrittsalter für Frauen niedriger (62,7 Jahre) als für Männer (63,6 Jahre).

Die Entscheidung zum Eintritt in den Ruhestand hängt nicht nur vom gesetzlichen Renteneintrittsalter $\mathrm{ab}$. $\mathrm{Zu}$ den relevanten Faktoren gehören körperliche Gesundheit, Bedingungen am Arbeitsmarkt, Großzügigkeit und Steuerbelastung des Renteneinkommens, private Ersparnisse, Familienverpflichtungen, Arbeitsmöglichkeiten für den Ehepartner und Einstellung am Arbeitsplatz gegenüber älteren Menschen.

Das durchschnittliche effektive Renteneintrittsalter liegt unter dem gesetzlichen Rentenalter (Abbildung SS6.1). Es gibt Ausnahmen. In Japan, Korea und Mexiko liegt das durchschnittliche Renteneintrittsalter mehr als fünf Jahre über dem gesetzlichen Alter. Mexikanische Frauen arbeiten zehn Jahre über das gesetzliche Alter hinaus und mexikanische Männer acht Jahre. Koreanische Männer arbeiten ebenfalls mehr als zehn Jahre über das Renteneintrittsalter hinaus. Desgleichen in der Türkei und Griechenland, wo Frauen und Männer einige Jahre über das gesetzliche Alter hinaus arbeiten.
Das durchschnittliche effektive Renteneintrittsalter variiert im Ländervergleich mehr als das gesetzliche Rentenalter. Das effektive Renteneintrittsalter liegt in Mexiko und Korea mit bei oder über 70 Jahren am höchsten. Am niedrigsten liegt es in Ungarn, Belgien, der Slowakischen Republik, Luxemburg, Österreich und Frankreich, wo die Menschen gegen Ende 50 aus dem Arbeitsleben ausscheiden und in Rente gehen.

Das effektive Renteneintrittsalter steigt in vielen Ländern, insbesondere für Frauen (Abbildung SS6.2). In den vergangenen 30 Jahren war das effektive Alter des Eintritts in den Ruhestand in den meisten OECD-Ländern deutlich gesunken, außer in Japan und Korea. Dieser Trend wurde bedingt durch die steigende Lebenserwartung, ein dynamisches Arbeitsmarktumfeld und stärkere finanzielle Arbeitsanreize für ältere Menschen unterbrochen oder umgekehrt. Politikveränderungen zur Erhöhung des gesetzlichen Rentenalters könnten in manchen Ländern eine Rolle gespielt haben. Zwischen 1999 und 2002 sowie 2002 und 2007 ist das effektive Alter des Eintritts in den Ruhestand für Frauen in 13 OECD-Ländern und für Männer in sieben OECD-Ländern um mehr als ein Jahr gestiegen. Zu den Ländern mit den höchsten Anstiegsraten beim effektiven Renteneintritt für Frauen gehören Mexiko, Neuseeland und die Türkei. Bei den Männern stieg das Alter deutlich in Korea, Neuseeland und Schweden. Es gibt jedoch Länder, in denen die Lebenserwartung steigt und das effektive Rentenalter sinkt (zum Beispiel Island bei den Frauen und Dänemark bei den Männern). Dieses Muster kann Probleme im Hinblick auf die dauerhafte fiskalische Tragfähigkeit nach sich ziehen.

Es gibt kaum eine Beziehung zwischen dem tatsächlichen Alter bei Austritt aus dem Erwerbsleben und der Lebenserwartung der Menschen auf Länderebene. Selbst das Verhältnis zwischen dem gesetzlichen Mindestalter - ein wesentliches Politikinstrument - und dem tatsächlichen Alter des Eintritts in den Ruhestand ist im Ländervergleich alles andere als deutlich (die Korrelation beträgt lediglich $0,35)$.

\section{Weitere Informationen}

OECD (2007), Renten auf einen Blick 2007: Staatliche Politik im OECD-Ländervergleich, Paris.

\section{Anmerkungen zu den Abbildungen}

Abbildungen S56.1 und SS6.2: Das durchschnittliche effektive Renteneintrittsalter wird von den beobachteten Veränderungen bei den Erwerbsquoten über einen Zeitraum von fünf Jahren für aufeinanderfolgende Kohorten von Arbeitnehmern ab dem Alter von 40 Jahren abgeleitet (nach Altersgruppen von jeweils fünf Jahren). Die Lebenserwartung bezieht sich auf das Jahr 2005, und Veränderungen betreffen den Zeitraum 20002005. 
SS6.1. Das Alter bei Austritt aus dem Erwerbsleben liegt generell niedriger als das gesetzliche Renteneintrittsalter

Alter bei Austritt aus dem Erwerbsleben im Vergleich zum gesetzlichen Rentenalter, 2007

Frauen

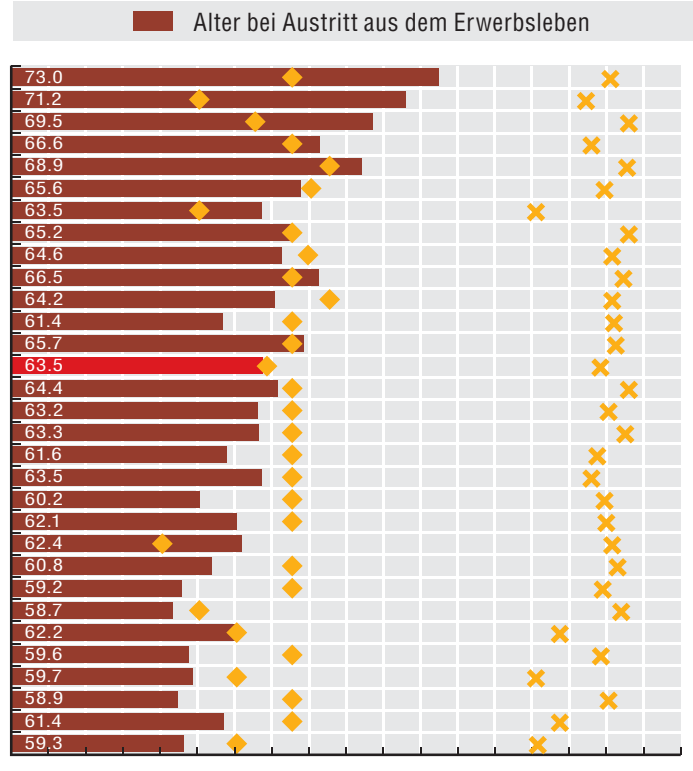

50525456586062646668707274767880828486
Lebenserwartung mit 65 Jahren, 2005

Männer

Lebenserwartung mit 65 Jahren $\quad$ Gesetzliches Rentenalter

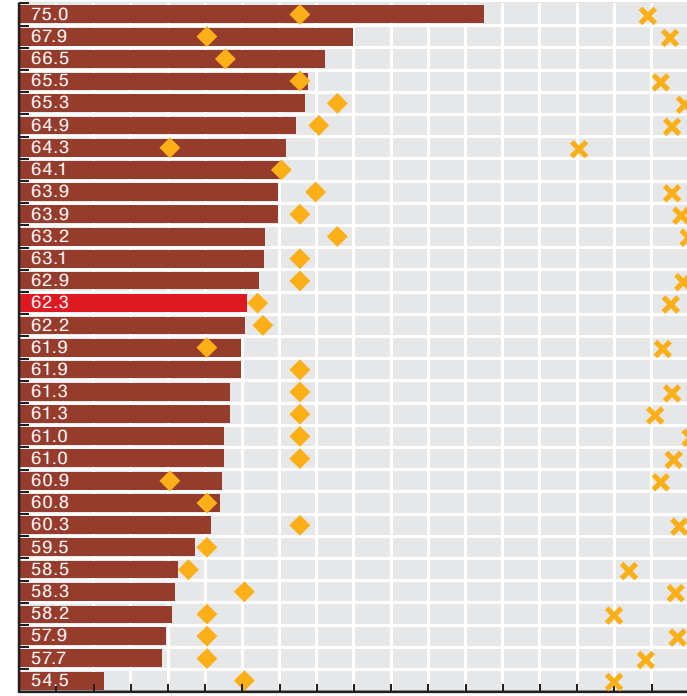

$505254565860626466 \quad 687072747678 \quad 80828486$

Mexiko
Korea
Japan
Portugal
Island
Irland
Türkei
Schweiz
Ver. Staaten
Neuseeland
Norwegen
Spanien
Schweden
OECD
Australien
Ver. Königreich
Kanada
Niederlande
Dänemark
Finnland
Deutschland
Griechenland
Italien
Luxemburg
Frankreich
Tschech. Rep.
Belgien
Ungarn
Österreich
Polen
Slowak. Rep.

Slowak. Rep.

SS6.2. Steigende Lebenserwartung im Vergleich zur Veränderung des gesetzlichen Rentenalters und des Alters bei Austritt aus dem Erwerbsleben

Alter bei Austritt aus dem Erwerbsleben und gesetzliches Rentenalter, 2002-2007 Frauen

Veränderung des Austrittsalters
Lebenserwartung mit 65 Jahren, 2000-2005 Männer

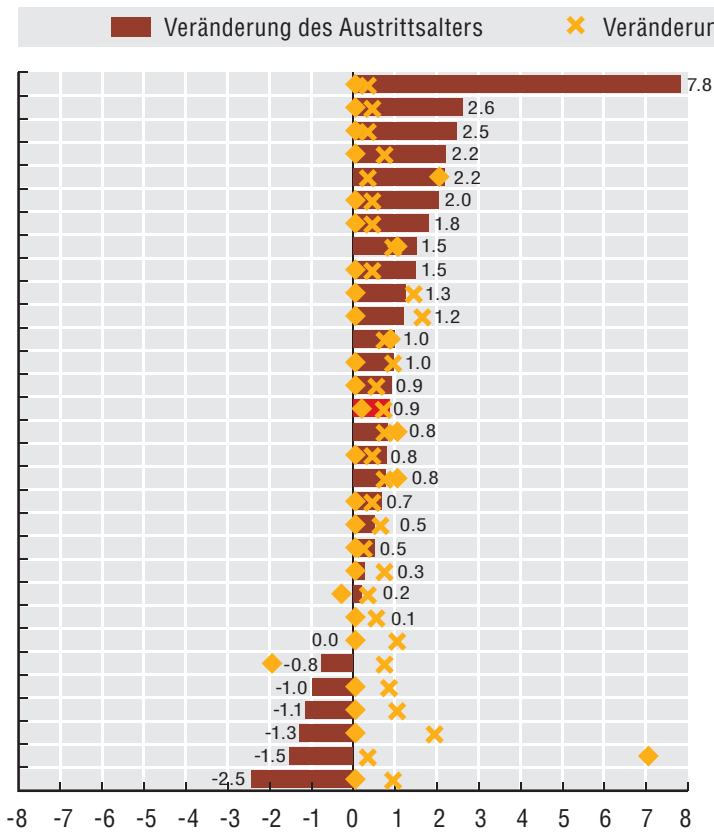

Veränderung des gesetzlichen Rentenalters

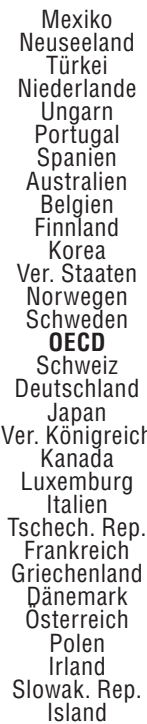

Island

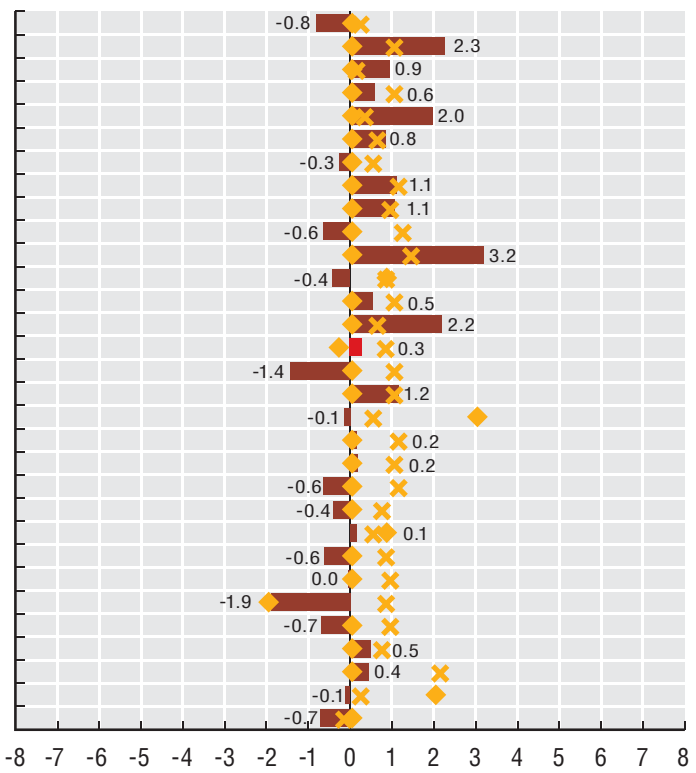

Quelle: OECD-Schätzungen nach europäischen und nationalen Arbeitskräfteerhebungen. 


\section{Definition und Messung}

Die Bildungsausgaben im Verhältnis zum Nettonationaleinkommen (NNE) dienen als Messgröße dafür, wie viel in das Humankapital investiert wird (ohne Zeitinput der Eltern sowie Bildung oder Ausbildung am Arbeitsplatz) im Verhältnis zu den gesamten monetären Ressourcen, die der Gesellschaft zur Verfügung stehen. Dieser Indikator misst sowohl die öffentlichen als auch die privaten Ausgaben für Bildungseinrichtungen (inklusive öffentlicher Subventionen) und die Ausgaben der Familien, sofern sie sich in Zahlungen an Bildungseinrichtungen äußern. Die Daten zu den Ausgaben beinhalten nicht die Subventionen für Lebenshaltungskosten der Studenten, subventionierte Studentenkredite und andere direkte private Ausgaben (z.B. für Schulbücher oder Schultransport). Ausgaben für Vorschulbildung oder Kinderbetreuung, die eine bildende Komponente enthalten kann, sind ebenfalls nicht berücksichtigt.

Ländervergleiche der Bildungsausgaben pro Schüler/ Student beziehen sich auf Kaufkraftparitäten-Wechselkurse und Messgrößen des Nettonationaleinkommens (vgl. „Definition und Messung“ für GE1. Nettonationaleinkommen). Zu beachten ist, dass die Verwendung des NNE statt des BIP als Nenner den Anteil der Bildungsausgaben erhöht und die Rangfolge mancher Länder im Vergleich zu der Analyse aus Bildung auf einen Blick 2008: OECD-Indikatoren verändert.

Die OECD-Länder geben im Durchschnitt 7\% ihres Nettonationaleinkommens für Bildung aus (Abbildung SS7.1). Von diesen $7 \%$ entfällt etwa 1 Prozentpunkt auf private Ausgaben. Der proportionale Gesamtbetrag der Ausgaben variiert, von über $8 \%$ in Island, Dänemark, Neuseeland, Korea und den Vereinigten Staaten bis zu unter $5,5 \%$ in Italien, Spanien und Griechenland. Die Bildungsausgaben werden durch die Anzahl der Kinder beeinflusst, und alle drei Länder mit niedrigen Ausgaben haben im OECD-Vergleich niedrige Geburtenziffern. Ebenfalls interessant ist die Beobachtung, dass die privaten Bildungsausgaben in Finnland am niedrigsten sind und in Korea am höchsten.
Dabei sind dies die beiden Länder mit den besten Ergebnissen bei der PISA-Studie, die ihre Gesamtausgaben sehr effektiv kombinieren, jedoch auf zwei ganz unterschiedliche Arten.

Die Summe der Bildungsausgaben als Anteil des NNE ist in den OECD-Ländern zwischen 1995 und 2005 moderat gestiegen (Abbildung SS7.2). Ein starker Anstieg ist insbesondere in Island (etwa 2 Prozentpunkte des NNE), dem Vereinigten Königreich und Mexiko (beide etwa 1 Prozentpunkt) zu verzeichnen. Über denselben Zeitraum wurden jedoch in zehn Ländern auch sinkende Ausgaben beobachtet, darunter in Kanada, Finnland und Norwegen.

Es gibt eine positive Beziehung zwischen kumulativen Bildungsausgaben im Alter von 6-15 Jahren und den PISA-Mathematikergebnissen des jeweiligen Landes (Abbildung SS7.3). Diese Beziehung ist zwar stabil, aber von relativ moderater Stärke (die Korrelation beträgt 0,47). Die überdurchschnittlichsten Ergebnisse auf der Basis ihres Nettonationaleinkommens erzielen die beiden Länder mit den besten PISA-Noten, Korea und Finnland. Die Niederlande und Neuseeland erreichen ebenfalls überdurchschnittliche Ergebnisse. Unterdurchschnittliche Ergebnisse im Verhältnis zu ihrem Nationaleinkommen erreichen u.a. die Türkei, Mexiko, Italien und die Vereinigten Staaten. Die Bildungsausgaben für Kinder unter 6 Jahren sind zwar nicht in der Abbildung SS7.2 enthalten, doch sind sie in der Regel in den nordischen Ländern hoch, diese Länder schneiden allerdings mit der Ausnahme Finnlands nicht besser ab als prognostiziert. Zusätzlich sind entsprechende Ausgaben für frühkindliche Bildung in Korea sehr niedrig, das Land ist jedoch bei PISA besonders erfolgreich. Es ist unwahrscheinlich, dass die Nichtberücksichtigung der frühkindlichen Bildungsausgaben einen wesentlichen Grund für die bescheidene Korrelation darstellt.

\section{Weitere Informationen}

OECD (2008), Bildung auf einen Blick 2008: OECD-Indikatoren, W. Bertelsmann Verlag.

\section{Anmerkungen zu den Abbildungen}

Abbildung SS7.3: PISA: Internationale Schulleistungsstudie der OECD. 
SS7.1. Anteil der gesamten Bildungsausgaben am Nettonationaleinkommen In Prozent, 2005

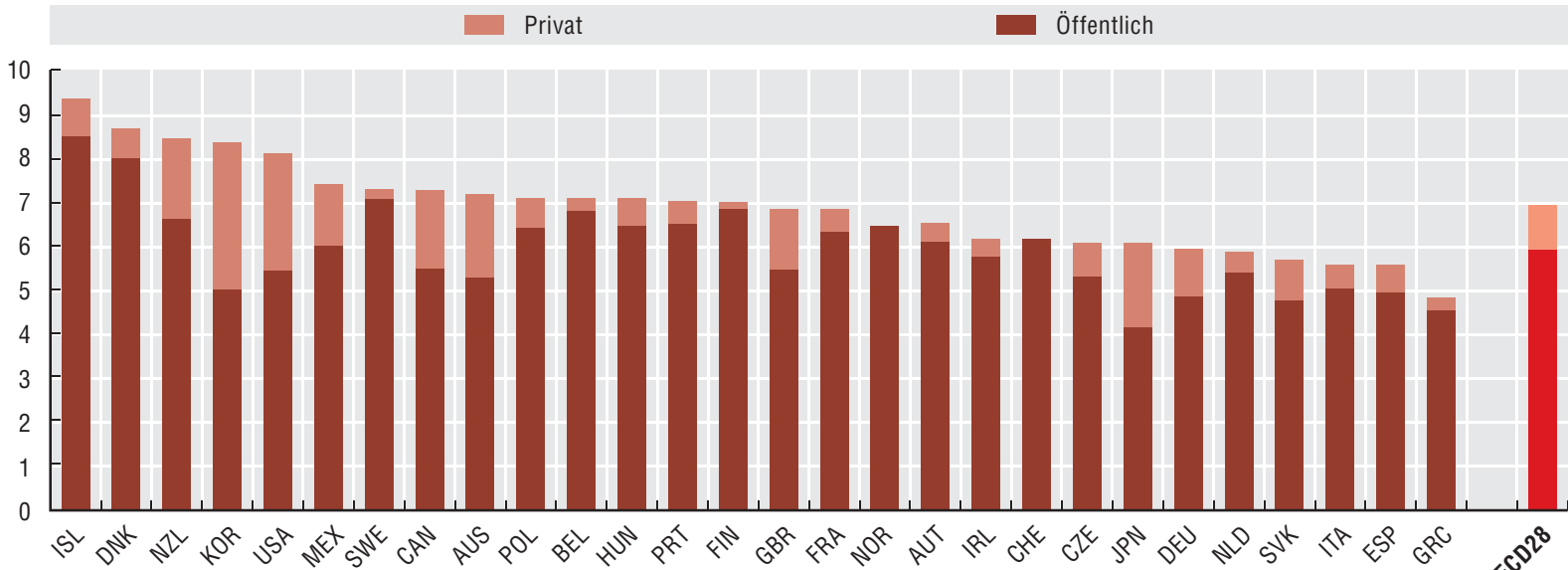

SS7.2. Anteil der gesamten Bildungsausgaben am Nettonationaleinkommen, 1995-2005 In Prozent, 1995, 2000, 2005

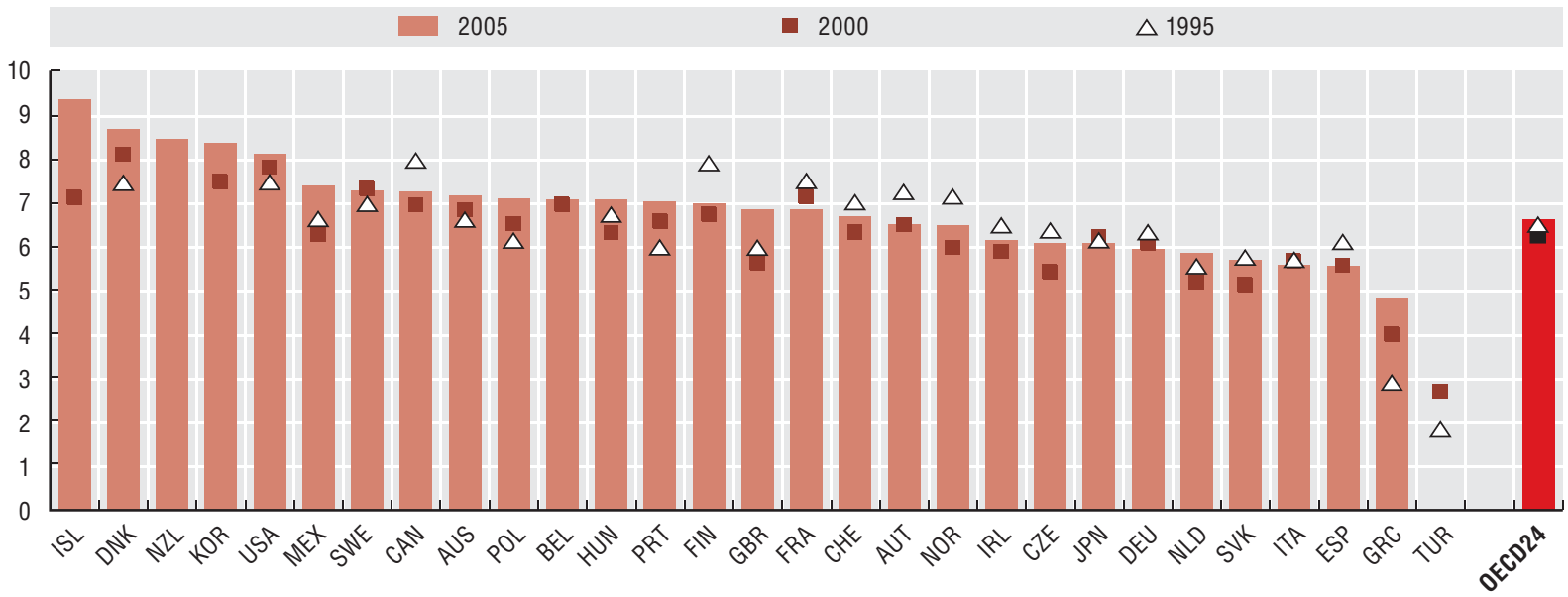

SS7.3. Kumulierte Bildungsausgaben im Verhältnis zu den PISA-Mathematikergebnissen von 2006

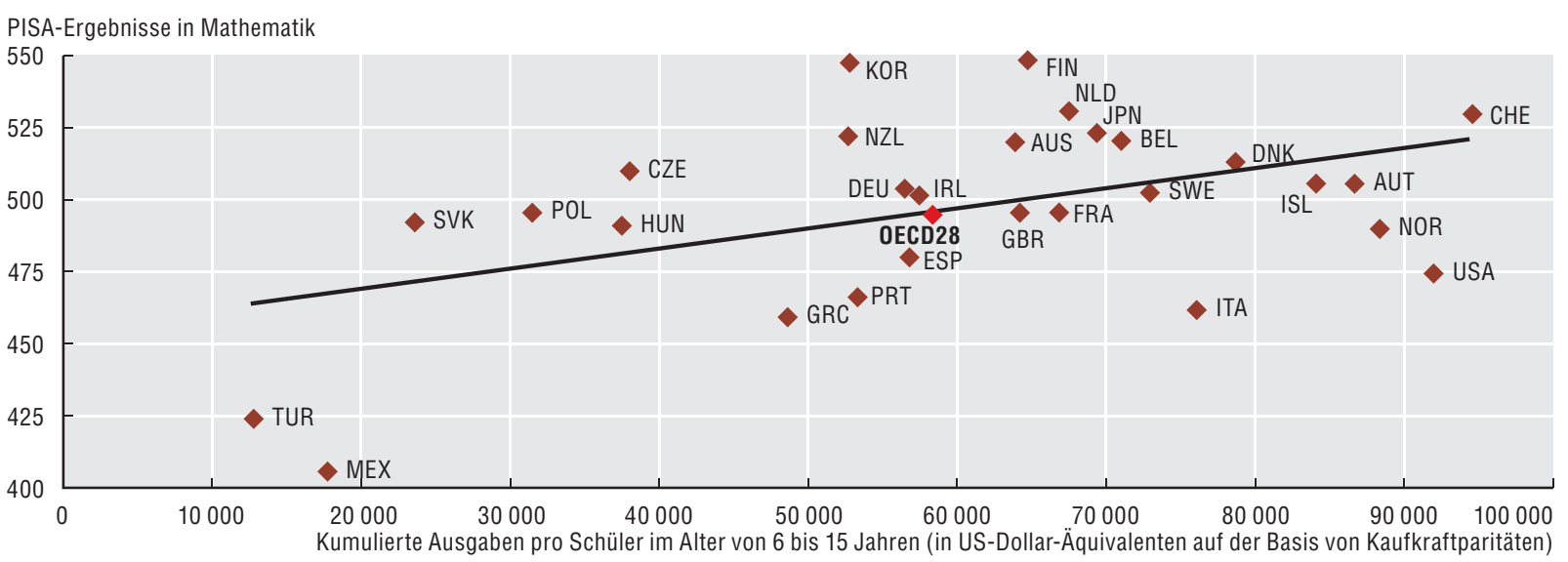




\section{Definition und Messung}

Die Messgrößen der Einkommensungleichheit basieren auf Daten über die verfügbaren Einkommen der privaten Haushalte. Das verfügbare Einkommen ist das Haushaltsbruttoeinkommen nach Abzug von direkten Steuern und Arbeitnehmerbeiträgen zur Sozialversicherung. Ausgeklammert bleiben Sachleistungen, die die Haushalte vom Staat und privaten Einrichtungen erhalten, Verbrauchsteuern und kalkulatorische Einkommensströme auf Grund von Wohneigentum und anderen Realvermögenswerten. Das Einkommen des Haushalts wird den einzelnen Haushaltsmitgliedern zugeordnet. Das Haushaltseinkommen wird bereinigt, um der Haushaltsgröße Rechnung zu tragen, wobei eine einheitliche Äquivalenzskala von 0,5 unterstellt wird. Der wichtigste verwendete Einkommensverteilungsindikator ist der Gini-Koeffizient. Die Werte des Gini-Koeffizienten liegen zwischen 0 im Falle einer „vollkommenen Gleichverteilung“ und 1 im Falle einer „vollkommenen Ungleichverteilung“ (das gesamte Einkommen geht an den Bevölkerungsteil mit dem höchsten Einkommen). Es wird auch ein Verhältnis zwischen Einkommensdezilen, das Verhältnis zwischen dem oberen Wert des neunten Dezils und dem des ersten Dezils, verwendet.

Die hier zu Grunde gelegten Daten wurden der OECD von nationalen Referenten zur Verfügung gestellt. Sie basieren auf einheitlichen Methoden und Definitionen, die auf die nationalen Mikrodatenreihen angewendet wurden. Dadurch verbesserte sich zwar die Vergleichbarkeit, doch weichen die nationalen Datenreihen derart voneinander ab, dass eine Standardisierung nicht ohne weiteres erreicht wird.

Die Einkommensungleichheit ist in den OECD-Ländern sehr unterschiedlich. Mitte der 2000er Jahre war der GiniKoeffizient der Einkommensungleichheit in Dänemark und Schweden am niedrigsten und in Mexiko und der Türkei am höchsten (Abbildung EQ1.1). Der Gini-Koeffizient für das Land mit der größten Ungleichheit ist doppelt so hoch wie der Wert des Landes mit der geringsten Ungleichheit. Auch das Verhältnis zwischen den Dezilen P90/P10 lässt große Disparitäten erkennen, wobei der Wert für das höchste Einkommensdezil in Dänemark, Schweden und Norwegen weniger als dreimal so hoch ist wie der des unteren Dezils, dagegen über rund sechsmal so hoch in den Vereinigten Staaten, in Portugal und der Türkei sowie mehr als achtmal so hoch in Mexiko. Die Korrelation zwischen den beiden in Abbildung EQ1.1 verwendeten summarischen Messgrößen der Einkommensungleichheit ist hoch (etwa 0,95).

Die Einkommensungleichheit hat generell zugenommen. Aus wirtschaftspolitischer Perspektive sind Veränderungen der Einkommensungleichheit bei Ländervergleichen sachdienlicher als Niveauvergleiche. Abbildung EQ1.2 zeigt Prozentpunktveränderungen des Gini-Koeffizienten in drei verschiedenen Zeiträumen. Im Gesamtzeitraum von Mitte der 1980er bis Mitte der 2000er Jahre (rechter Teil von Abbildung EQ1.2) steigt die Ungleichheit in 19 von 24 Ländern, für die Datenmaterial verfügbar ist. Am stärksten erhöht sich die Ungleichheit in Finnland, Neuseeland und Portugal. Zu einem Rückgang kam es in Frankreich, Griechenland und der Türkei sowie in Irland und Spanien (wo die Daten bis 2000 reichen). Der OECD-durchschnittliche Anstieg des Gini-Koeffizienten entspricht einem hypothetischen 4\%igen Transfer des Durchschnittseinkommens von Personen in der unteren Hälfte der Einkommensverteilung zu Personen in der oberen Hälfte. Insgesamt ist diese Zunahme der Ungleichheit zwar moderat, aber signifikant.

Die Einkommensungleichheit hat sich im Zeitraum von Ende der 1980er bis Mitte der 1990er Jahre in vielen Ländern vergrößert, danach wies das Muster stärkere Variationen auf. Der mittlere und linke Teil von Abbildung EQ1.2 lässt erhebliche Unterschiede in Bezug auf die Entwicklung der Ungleichheit erkennen, sowohl beim Vergleich zwischen Ländern als auch zwischen Zeiträumen. In den zehn Jahren von Mitte der 1980er bis Mitte der 1990er Jahre (linker Teil) ist das dominierende Muster zunehmender Ungleichheit in Mexiko, Neuseeland und der Türkei sowie in mehreren anderen Ländern besonders deutlich. Im darauffolgenden Zehnjahreszeitraum sind die Unterschiede im Hinblick auf Veränderungen der Ungleichheit größer (mittlerer Teil). In vielen Ländern - vor allem in Deutschland, Finnland, Kanada, Norwegen, Portugal, Schweden und den Vereinigten Staaten - ist ein höherer Grad an Ungleichheit festzustellen. In diesem Zeitraum hat sich hingegen die Ungleichheit in Mexiko und der Türkei sowie in kleineren Ländern wie Australien, Griechenland, Irland, die Niederlande und das Vereinigte Königreich stark verringert.

\section{Weitere Informationen}

OECD (2008), Mehr Ungleichheit trotz Wachstum? Einkommensverteilung und Armut in OECD-Ländern, Paris (www.oecd.org/ els/social/inequality).

\section{Anmerkungen zu den Abbildungen}

Abbildung EQ1.1: Die Länder sind in aufsteigender Reihenfolge von links nach rechts nach dem Gini-Koeffizienten aufgeführt. Das verwendete Einkommenskonzept ist das des verfügbaren monetären Haushaltseinkommens, um die Haushaltsgröße bereinigt mit einer Elastizität von 0,5.

Abbildung EQ1.2: Im ersten Teil beziehen sich die Daten für Portugal, die Tschechische Republik und Ungarn und die alten Bundesländer Deutschlands auf Veränderungen von ungefähr 1990 bis Mitte der 1990er Jahre (für Australien, Polen und die Schweiz sind keine Daten verfügbar). Im zweiten Teil beziehen sich die Daten für Belgien, Irland, Österreich, Portugal, Spanien und die Tschechische Republik (wo die Daten von 2005 auf der Basis von EU-SILC mit denen früherer Jahre nicht vergleichbar sind) auf die Veränderungen von Mitte der 1990er Jahre bis ungefähr 2000. OECD24 bezieht sich auf den einfachen Durchschnitt der OECD-Länder mit Daten, die sich über den gesamten Zeitraum erstrecken (alle o.a. Länder mit Ausnahme Australiens), OECD22: ohne Mexiko und die Türkei. 
EQ1.1. Die Einkommensungleichheit ist in den OECD-Ländern sehr unterschiedlich

Gini-Koeffizient und Dezilverhältnis (P90/P10), Mitte 2000er Jahre

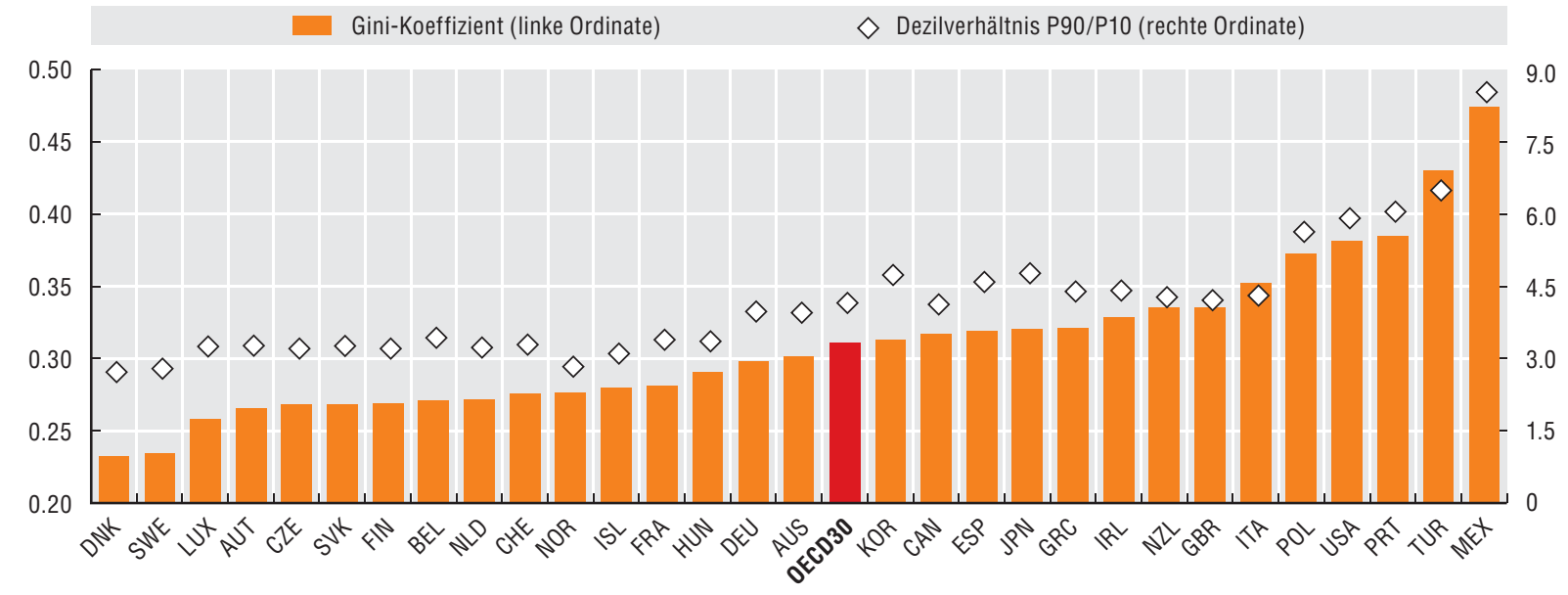

EQ1.2. Die Einkommensungleichheit hat generell zugenommen

Prozentpunktveränderungen des Gini-Koeffizienten
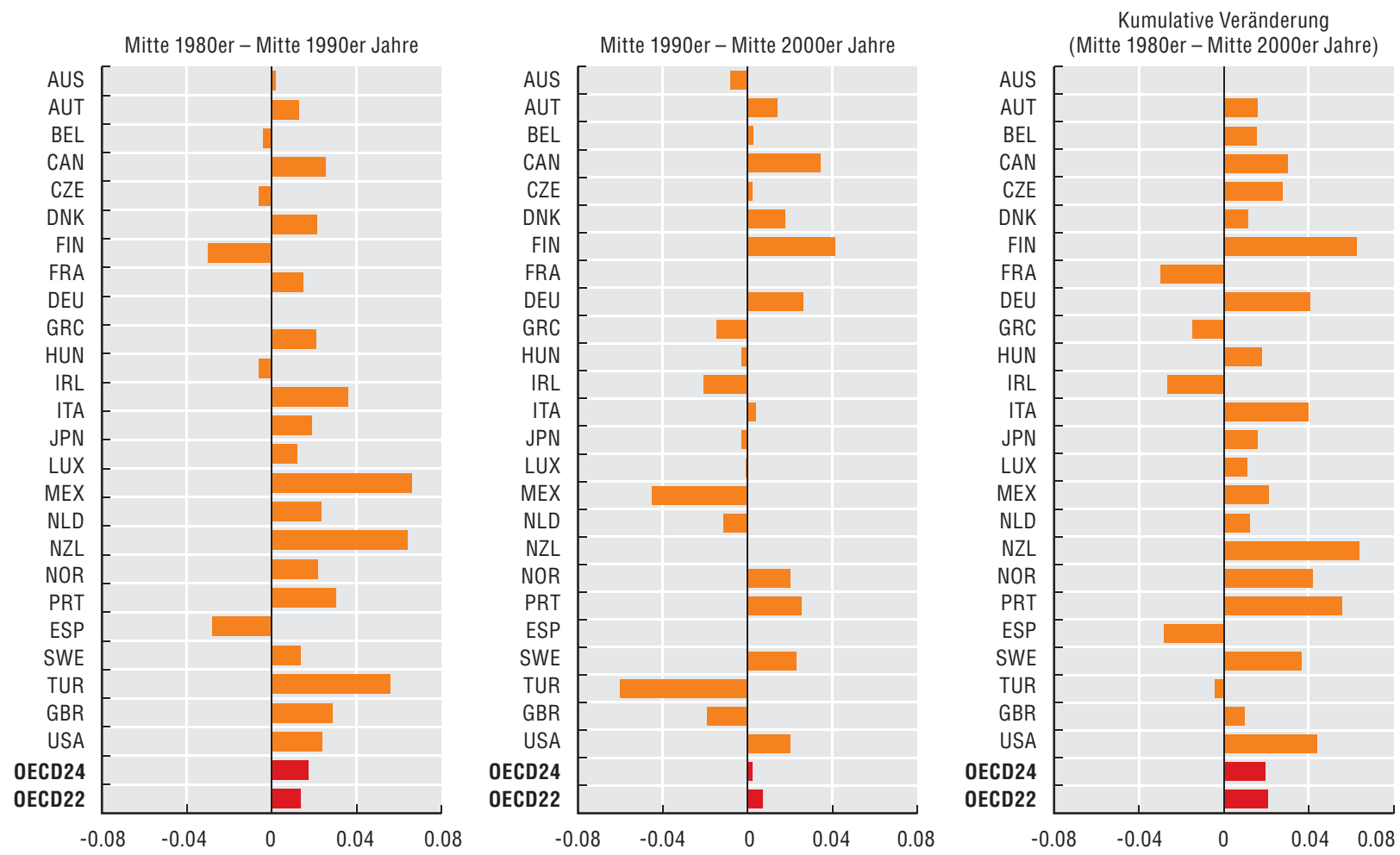

Quelle: OECD (2008), Mehr Ungleichheit trotz Wachstum? Einkommens-

verteilung und Armut in OECD-Ländern, Paris

(www.oecd.org/els/social/inequality). 


\section{Definition und Messung}

Die Vermeidung materieller Härten ist eines der Hauptziele der Sozialpolitik, das zuweilen mittels eines in der Verfassung verankerten Rechts auf einen „annehmbaren Lebensstandard“ explizit genannt wird. Das, was als „annehmbarer Lebensstandard“ gilt, variiert jedoch zwischen den Ländern und im Zeitverlauf. Daher gibt es in den OECD-Ländern keine gemeinsam vereinbarte Messgröße der Armut. Wie bei der Einkommensungleichheit ist der Ausgangspunkt für die Messung das für die OECD von Experten aus den einzelnen Ländern bereitgestellte äquivalenzgewichtete verfügbare Haushaltseinkommen (vgl. „Definition und Messung“ unter EQ1. Einkommensungleichheit). Als arm werden Menschen eingestuft, wenn ihr äquivalenzgewichtetes Haushaltseinkommen weniger als der Hälfte des Medianeinkommens in jedem Land entspricht. Auf Grund der Verwendung einer relativen Einkommensschwelle weisen reichere Länder höhere Armutsschwellen auf. Durch die höheren Armutsschwellen in reicheren Ländern wird dem Konzept Rechnung getragen, dass eine Person als nicht arm gilt, die in der Lage ist, Zugang zu Gütern und Dienstleistungen zu haben, die in einem beliebigen Land als „üblich“ gelten.

Armut wird unter dem Gesichtspunkt der Armutsquote und der Armutslücke betrachtet. Die Armutsquote ist definiert als der zahlenmäßige Anteil der Personen, die mit ihrem Einkommen unter der Armutsgrenze liegen. An der Armutslücke wird der Grad gemessen, in dem das Einkommen der Armen unter der Armutsgrenze liegt. Die Armutslücke ist der (in Prozent der Armutsschwelle ausgedrückte) Abstand zwischen dem Medianeinkommen aller Armen und der Armutsgrenze.

In den OECD-Ländern betrug die durchschnittliche Armutsquote Mitte der 2000 er Jahre etwa $11 \%$ (Abbildung EQ2.1). Zwischen den Ländern bestehen deutliche Unterschiede. So liegen die Armutsquoten in Polen, Korea, Irland und Japan bei rd. 15\% und in Mexiko, der Türkei und den Vereinigten Staaten bei $17-18 \%$, in Dänemark, Schweden und der Tschechischen Republik hingegen unter $6 \%$. Eine unterschiedliche Höhe der Armutsgrenze verändert die Armutsquote ganz erheblich. Auf der Basis einer bei $40 \%$ des Medianeinkommens angesetzten Armutsgrenze sinkt die durchschnittliche Armutsquote im OECD-Raum auf rd. 6\%. Bei einer Armutsgrenze von $60 \%$ des Medianeinkommens erhöht sich die durchschnittliche Armutsquote auf rd. 17\%.

In den OECD-Ländern betrug die durchschnittliche Armutslücke Mitte der 2000er Jahre 29\% (Abbil- dung EQ2.1). Am größten ist die Armutslücke mit einem Abstand von rd. 38\% zur Armutsgrenze in Mexiko, den Vereinigten Staaten und der Schweiz. Im unteren Bereich des Spektrums, in Finnland, Luxemburg, den Niederlanden und Belgien, liegt sie bei etwa $20 \%$.

Länder mit höheren Armutsquoten haben in der Regel größere Armutslücken. Die Messungen beider Größen ergeben eine hohe positive Korrelation von 0,60. Bei dieser allgemeinen Tendenz gibt es einige deutliche Ausreißer. In einigen Ländern, die sich durch relativ niedrige Armutsquoten auszeichnen, wie insbesondere Island und die Schweiz, liegen die Armutslücken mit 30\% oder mehr weit über dem OECD-Durchschnitt. Ebenso liegen in einigen Ländern, wie z.B. Australien, Griechenland, Irland und Kanada, die Armutsquoten über dem OECD-Durchschnitt zu verzeichnen haben, die Armutslücken unter dem Durchschnittswert.

Die Armutsquoten haben sich im Zeitraum von Mitte der 1980er bis Mitte der 2000er Jahre generell erhöht (Abbildung EQ2.2). Die Armutsquoten sanken in acht und erhöhten sich in 16 Ländern. Der stärkste Rückgang war in Belgien und Mexiko zu verzeichnen, während die übrigen Länder eine leichte Abnahme um rd. 1 Prozentpunkt oder weniger aufwiesen. Die Länder mit der stärksten Zunahme - zwischen 4 und 5 Prozentpunkten - waren Deutschland, Irland, Neuseeland und die Niederlande. Im Hinblick auf die Unterschiede bei der Zunahme der Armut zwischen den Zeiträumen von Mitte der 1980er bis Mitte der 1990er Jahre und Mitte der 1990er bis Mitte der 2000er Jahre betrifft, tritt kein eindeutiges Muster zu Tage. Der Anstieg betrug in jedem Teilzeitraum etwa 0,6 Prozentpunkte.

\section{Weitere Informationen}

OECD (2008), Mehr Ungleichheit trotz Wachstum? Einkommensverteilung und Armut in OECD-Ländern, Paris (www.oecd.org/ els/social/inequality).

\section{Anmerkungen zu den Abbildungen}

Abbildung EQ2.2: Die Daten in der linken Abbildung beziehen sich für Portugal, die Tschechische Republik und Ungarn auf Veränderungen der Armutsquote von ungefähr 1990 bis Mitte der 1990er Jahre; für Australien und die Schweiz sind keine Daten verfügbar. Die Daten in der mittleren Abbildung beziehen sich für Belgien, Irland, Österreich, Portugal, Spanien und die Tschechische Republik (wo die Daten von 2005 auf der Basis von EU-SILC mit denen früherer Jahre nicht vergleichbar sind) auf die Veränderungen von Mitte der 1990er Jahre bis ungefähr 2000 und für die Schweiz auf Veränderungen von 2000 bis 2005. OECD24 bezieht sich auf den einfachen Durchschnitt der OECD-Länder, deren Daten sich über den gesamten Zeitraum erstrecken (alle o.a. Länder mit Ausnahme Australiens und der Schweiz). 
EQ2.1. OECD-Länder mit hohen Armutsquoten haben in der Regel große Armutslücken

Armutsquote und Armutslücke, Mitte 2000er Jahre, Armutsgrenze 50\%

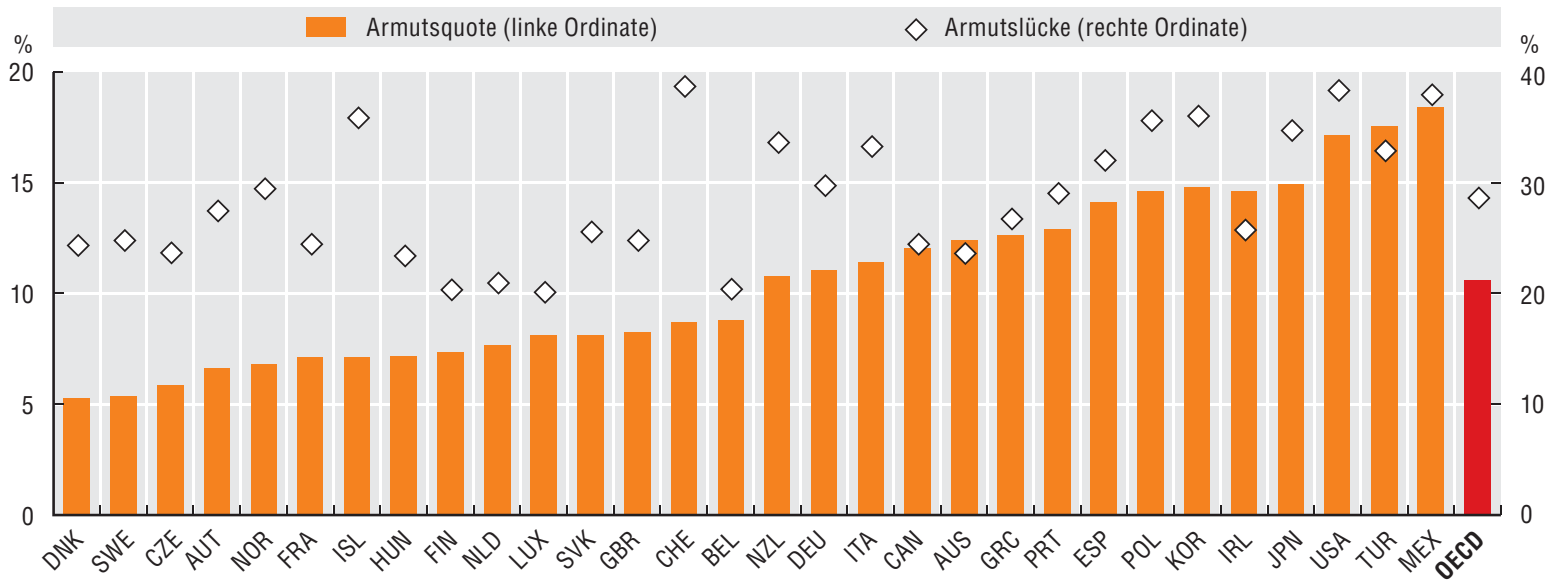

EQ2.2. Die Armutsquoten haben sich im Zeitraum Mitte der 1980er - Mitte der 2000er Jahre generell erhöht Prozentpunktveränderungen der Einkommensarmutsquote bei 50\% des Medianeinkommens in verschiedenen Zeiträumen
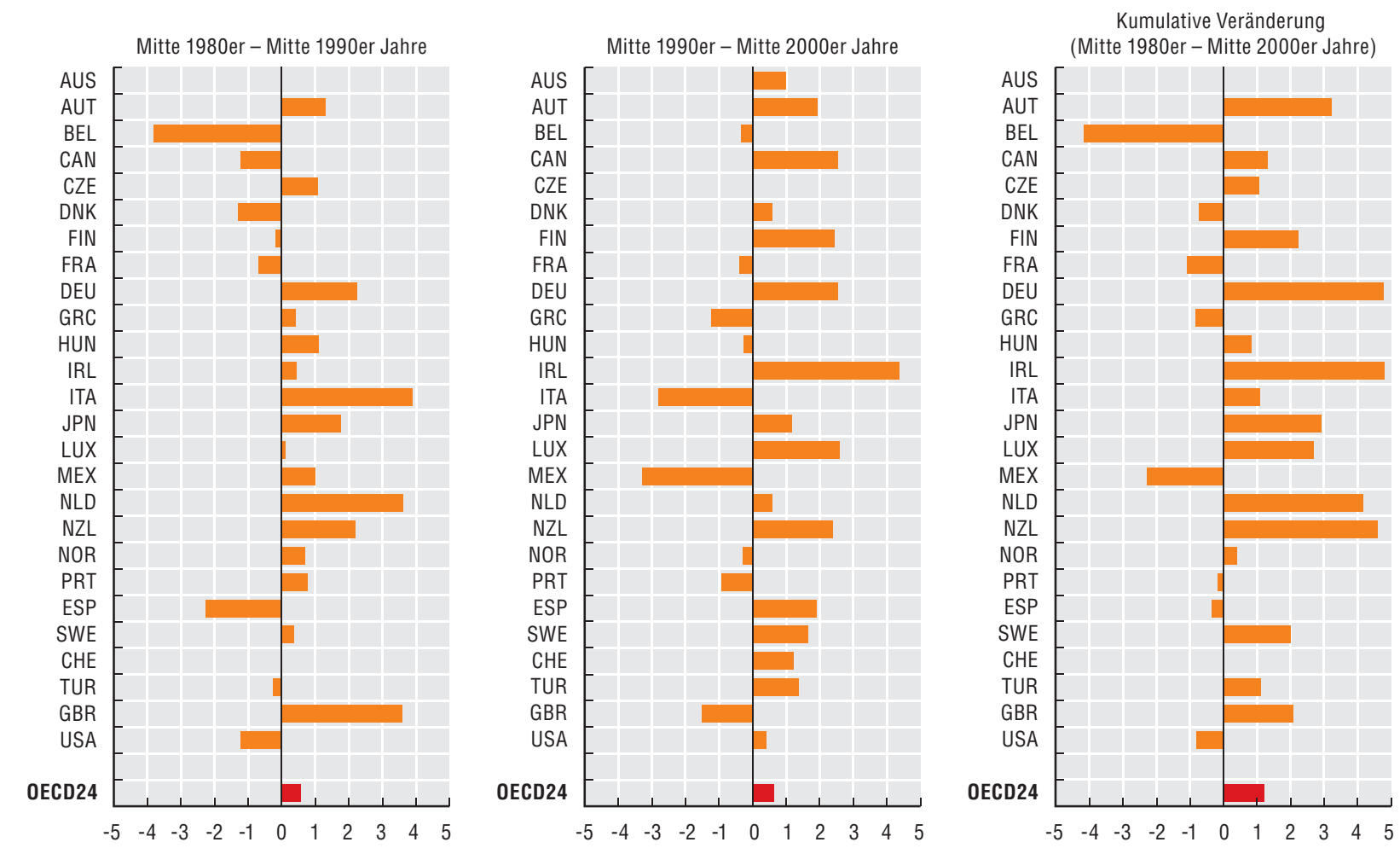

Quelle: OECD (2008), Mehr Ungleichheit trotz Wachstum?

Einkommensuerteilung und Armut in OECD-Ländern,

Paris (www.oecd.org/els/social/inequality). 


\section{Definition und Messung}

Kinder gelten definitionsgemäß als arm, wenn sie in Haushalten leben, deren äquivalenzgewichtetes verfügbares Einkommen weniger als $50 \%$ des Medianeinkommens eines gegebenen Landes entspricht (vgl. „Definition und Messung“ für EQ1. Einkommensungleichheit). Für Kinder, d.h. definitionsgemäß alle unter 18-Jährigen, wird unterstellt, dass sie an dem von den übrigen Haushaltsmitgliedern verdienten Einkommen teilhaben. Der hier verwendete Basisindikator für Kinderarmut ist die Armutsquote, gemessen als der Anteil der Kinder mit einem äquivalenzgewichteten Einkommen von weniger als 50\% des Medianeinkommens. Ebenfalls dargestellt sind die Armutsquoten aller in Haushalten mit Kindern lebenden Personen (d.h. einschließlich erwachsener Haushaltsmitglieder).

Mehr als bei anderen Altersgruppen werden die Schätzungen der Kinderarmut vom Elastizitätsgrad der Äquivalenzskala beeinflusst. Die hier gezeigten Schätzungen können auch eine gewisse Anzahl von Schülerinnen und Schülern (bis 18 Jahre) einschließen, die außerhalb des Elternhauses in Armut leben. Da bei den hier verwendeten Daten zudem der Haushalt als Grundeinheit dient, in dem das Einkommen zusammengelegt und auf die einzelnen Haushaltsmitglieder aufgeteilt wird, bleiben biologische und soziale Beziehungen zwischen den Haushaltsmitgliedern unberücksichtigt. Infolgedessen sind in der Kategorie „alleinstehende Erwachsene mit Kindern" neben Alleinerziehern auch andere Haushaltsstrukturen (wie z.B. ein mit Enkel oder Enkelin lebender Großelternteil) enthalten. Ebenso können „Eltern mit Kindern“ auch Alleinerzieherfamilien mit einschließen, die mit anderen Erwachsenen zusammen im selben Haushalt leben.

Die Kinderarmut gibt in den OECD-Ländern Anlass zu besonderer Besorgnis. Kinder haben über die materielle Situation ihrer Familie kaum Kontrolle. Die in der Kindheit erfahrene Armut kann zudem negative Auswirkungen auf die kognitive und soziale Entwicklung bis ins Erwachsenenalter hinein haben.

Die durchschnittliche Kinderarmutsquote im OECD-Raum lag Mitte der 2000er Jahre bei 12\% (Abbildung EQ3.1, linker Teil). Die Kinderarmutsquote liegt in Dänemark, Finnland, Schweden und Norwegen unter 5\% sowie in Frankreich und
Österreich unter $8 \%$. In den Vereinigten Staaten, Polen, Mexiko und der Türkei übersteigt sie $20 \%$.

Die Kinderarmutsquoten liegen im Allgemeinen über denen der Gesamtbevölkerung. Zu den Ländern, in denen Kinder mit geringerer Wahrscheinlichkeit arm sind als die Bevölkerung allgemein, gehören Australien, Dänemark, Finnland, Japan, Korea, Norwegen, Österreich und Schweden. Um mindestens 3 Prozentpunkte höher liegt die Kinderarmutsquote dagegen in Deutschland, Italien, Kanada, Luxemburg, Mexiko, Neuseeland, Polen, Portugal, Spanien, der Tschechischen Republik, der Türkei und den Vereinigten Staaten.

Die Kinderarmutsquote ist in den letzten zehn Jahren in allen OECD-Ländern gestiegen. Zu diesem leichten Anstieg kam es trotz der von mehreren Ländern angekündigten Ziele zur Bekämpfung der Kinderarmut und der Einführung anderer Maßnahmen mit Schwergewicht auf der Reduzierung der Zahl der in Armut lebenden Kinder. Stark gestiegen ist die Kinderarmut in der Türkei, in Deutschland, Luxemburg und Österreich. Ein bedeutender Rückgang ist in Mexiko, Italien und im Vereinigten Königreich zu beobachten (Abbildung EQ3.1, rechter Teil).

Die Armutswahrscheinlichkeit ist bei Kindern in Alleinerzieherfamilien und Arbeitslosenfamilien höher (Tabelle EQ3.2). Ein Großteil der Korrelation zwischen Alleinerziehern und Kinderarmut ist durch Arbeitslosigkeit bedingt. Bei Arbeitslosigkeit sind die Armutsquoten von Alleinerzieherfamilien im OECD-Durchschnitt mit denen von Familien vergleichbar, in denen die Kinder mit beiden Elternteilen aufwachsen. Mit zunehmender Kinderzahl steigen die Armutsquoten zudem etwas an.

\section{Weitere Informationen}

OECD (2008), Mehr Ungleichheit trotz Wachstum? Einkommensverteilung und Armut in OECD-Ländern, Paris (www.oecd.org/ els/social/inequality).

\section{Anmerkungen zu den Abbildungen und Tabellen}

Abbildung EQ3.1: Die Länder sind von oben nach unten in absteigender Reihenfolge ihrer Armutsquote Mitte der 2000er Jahre aufgeführt.

Tabelle EQ3.2: Die Veränderungen beziehen sich für Belgien, Irland, Österreich, Portugal, Spanien und die Tschechische Republik auf 1995-2000 sowie für die Schweiz auf 2000-2005. Für Frankreich beziehen sich die Niveaus auf EU-SILC, Veränderungen auf die Enquête nationale revenus fiscaux (ERF). 
EQ3.1. Die Kinderarmutsquoten sind in den letzten zehn Jahren in allen OECD-Ländern leicht gestiegen Armutsquoten auf der Basis einer Einkommensgrenze von 50\% des Medianeinkommens in Prozent und Prozentpunktveränderungen der Armutsquoten

Armutsquoten (in \%), Mitte der 2000er Jahre

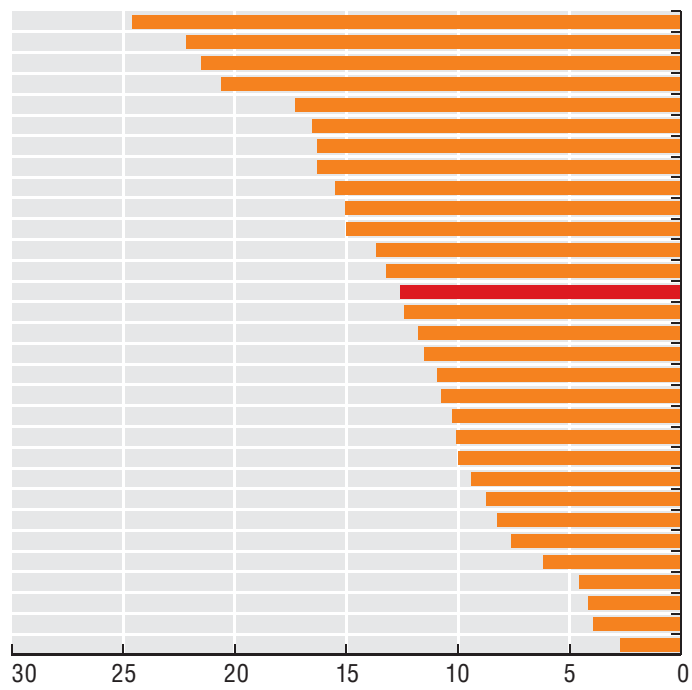

Prozentpunktveränderungen seit Mitte der 1990er Jahre
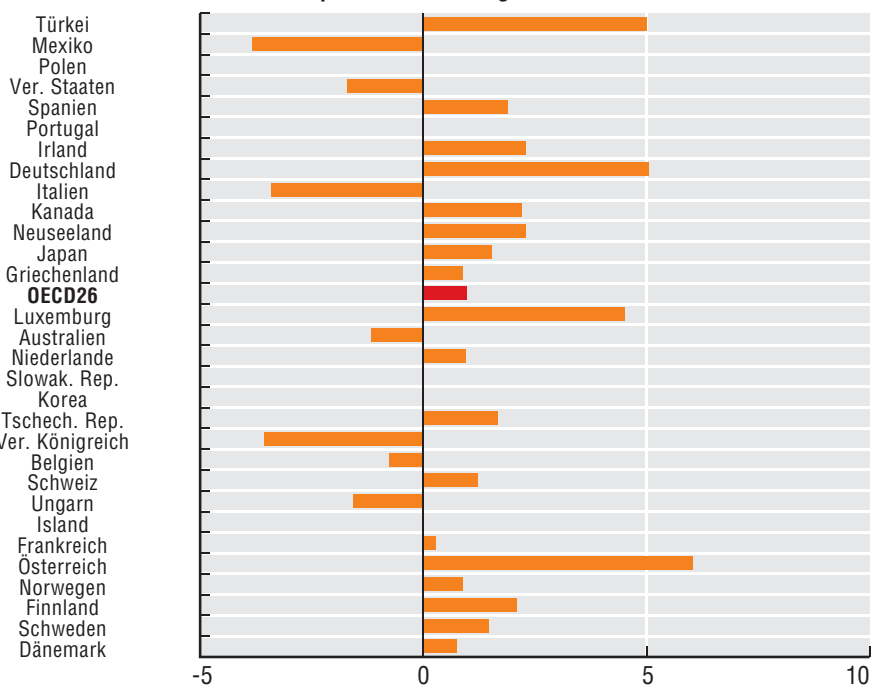

EQ3.2. Die Armutswahrscheinlichkeit ist bei Kindern in Alleinerzieherfamilien und in Arbeitslosenfamilien höher Armutsquoten auf der Basis einer Einkommensgrenze von 50\% des Medianeinkommens in Prozent und Prozentpunktveränderungen der Armutsquoten

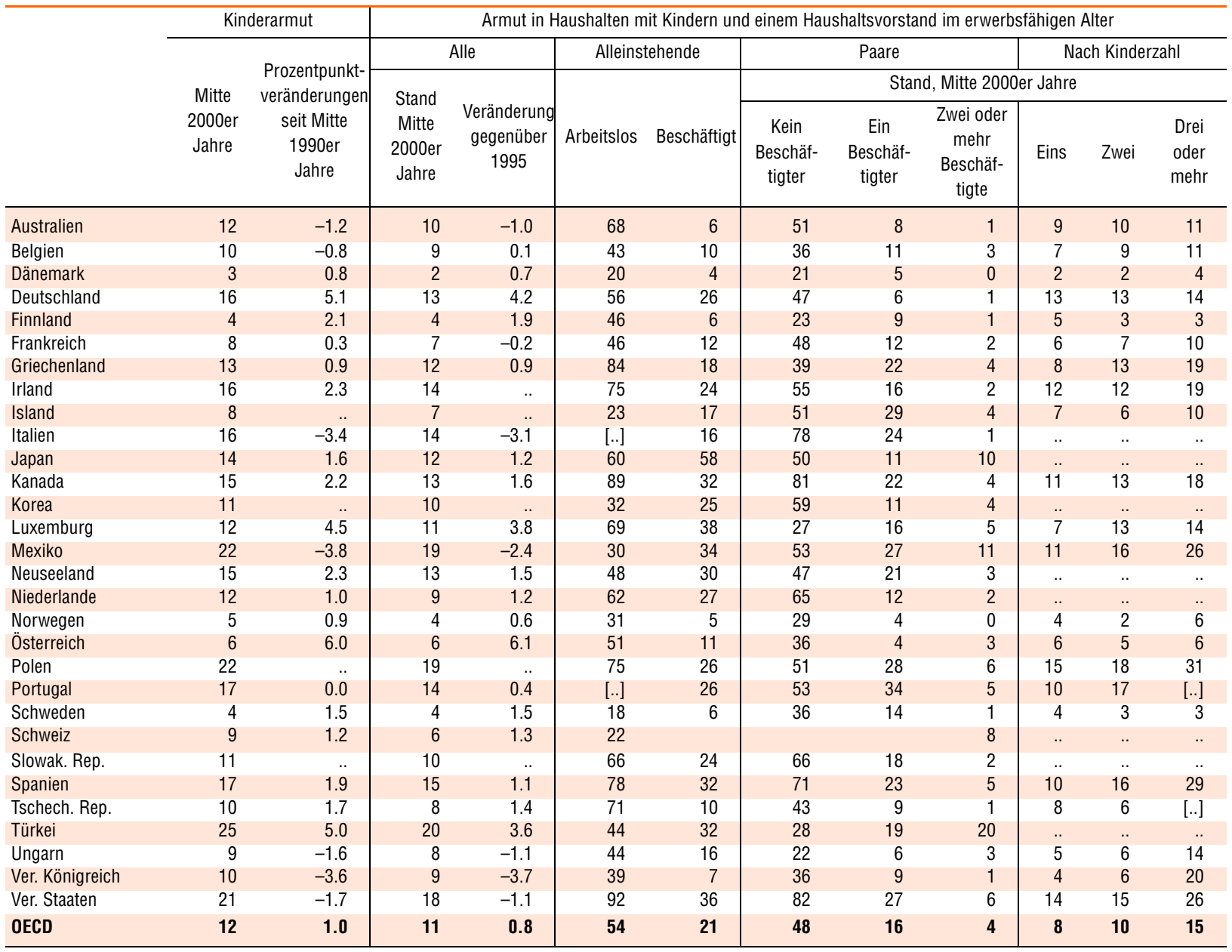

Quelle: OECD (2008), Mehr Ungleichheit trotz Wachstum? Einkommensverteilung

und Armut in OECD-Ländern, Paris (www.oecd.org/els/social/inequality). 


\section{Definition und Messung}

Im Vergleich zu Nachsteuer-Erwerbseinkommen wird mit den Nettoeinkommen von Sozialleistungsempfängern der finanzielle Anreiz für Arbeitslose gemessen, eine Beschäftigung aufzunehmen. Ein Vergleich dieser Messgröße mit den Einkommensgrenzen, die zur Identifizierung armer Familien herangezogen werden, gibt Aufschluss über die Frage, inwieweit Sozialleistungssysteme in der Lage sind, einen angemessenen Lebensstandard zu gewährleisten.

Die nachstehenden Indikatoren beziehen sich auf zwei verschiedene Zeiträume (2001 und 2005; der Zeitraum 2005 wurde gewählt, um das Datenmaterial aus der Veröffentlichung Mehr Ungleichheit trotz Wachstum? zu nutzen) und verwenden die OECD TaxBenefit-Modelle, um die Nettoeinkommen von zwei unterschiedlichen Kategorien von Ein-ElternFamilien den Schwellenwerten gegenüberzustellen, die zur Messung der Einkommensarmut herangezogen werden $(40 \%$, $50 \%$ und $60 \%$ des Haushaltsmedianeinkommens). Beide Ein-Eltern-Familien haben zwei Kinder im Alter von 6 und 4 Jahren. Im ersten Fall erhält die Familie weder Arbeitslosengeld noch bezieht sie Erwerbseinkommen. Die einzigen Familieneinkommensquellen sind daher familienbezogene monetäre Transferleistungen und Mindestleistungen der sozialen Sicherung. Der Leistungsbezug der Familie erstreckt sich über das ganze Steuerjahr. Die zweite Messwertreihe zeigt das Einkommen derselben Familie, jedoch bei Vollzeitbeschäftigung des Alleinerziehenden zum Mindestlohn. Alle Einkommensmesswerte beziehen sich auf das Einkommen nach Steuern, jedoch ohne Berücksichtigung beschäftigungsbedingter Kosten, z.B. für Kinderbetreuung. Die Haushaltsmedianeinkommen beider Jahre sind Mehr Ungleichheit trotz Wachstum? entnommen. Nähere Einzelheiten über die Methodik enthält die OECD-Veröffentlichung Benefits and Wages (2007).

Die festgelegten Mindestsicherungsleistungen liegen häufig unter den Schwellenwerten, die normalerweise bei Armutsvergleichen zwischen den OECD-Ländern verwendet werden (Abbildung EQ4.1). Leistungsempfänger, die nicht in der Lage sind, ein Erwerbseinkommen zu beziehen, sind in allen OECD-Ländern in hohem Maße armutsgefährdet. In allen 26 untersuchten OECD-Ländern, mit Ausnahme von 11 Ländern, lagen die Einkommen arbeitsloser Alleinerziehender 2005 unter einer Armutsschwelle von 50\%. In neun Ländern lagen die Einkommen 2001 an oder über einem Schwellenwert von 50\%.

Die Effektivität der Sozialhilfeleistungen und der mit ihnen verbundenen Transfers bei der Reduzierung der Armut von Alleinerziehenden konnte im Zeitraum 20012005 etwas gesteigert werden. Der Rückgang der Wirk- samkeit im Hinblick auf die Reduzierung der Armut von Alleinerziehenden ist gewöhnlich nicht auf eine Verringerung des nominalen Leistungsvolumens zurückzuführen, sondern auf die Tatsache, dass das Haushaltsmedianeinkommen schneller steigt als die Transferleistungen. Werden die Leistungen im Zeitverlauf mit geringer zeitlicher Frequenz und mit länderspezifisch unterschiedlichen Zyklen angepasst, könnte dies einen Teil der zwischen den Ländern bestehenden Unterschiede bei der Wirksamkeit erklären.

Für Alleinerziehende, die Mindestsicherungsleistungen erhalten und eine Beschäftigung aufnehmen, kann eine Niedriglohn-Vollzeittätigkeit solide Einkommensgewinne bringen. Abbildung EQ4.2 zeigt, dass Ein-Eltern-Familien bei Aufnahme einer Vollzeitbeschäftigung zum Mindestlohn (bei einem mit 50\% des Medianeinkommens angesetzten Schwellenwert) in Australien, Belgien, Irland, Japan, Neuseeland, den Niederlanden, Polen und im Vereinigten Königreich aus der Armut herausfinden. Die Wirksamkeit des Mindestlohns, die Armut von Alleinerziehenden zu verringern, erhöht sich - unter der Voraussetzung der Ausübung einer Vollzeittätigkeit - in Polen, Irland und Ungarn am stärksten und verringert sich am schnellsten in Australien, im Vereinigten Königreich und in Neuseeland. Ebenso wie bei den Leistungsmustern im Zeitverlauf könnte sich die Varianz durch das Zeitprofil der Mindestlohnanpassungen erklären.

Die Kombination von angemessenen Sicherheitsnetzen und starken Arbeitsanreizen erfordert ein gut austariertes System. Wie Abbildung EQ4.3 zeigt, werden Alleinerziehenden in Australien, Irland und im Vereinigten Königreich zwar relativ hohe Leistungen gezahlt, zugleich aber auch relativ starke Beschäftigungsanreize für den Schritt von der Inanspruchnahme von Mindestsicherungsleistungen zur Aufnahme einer Vollzeittätigkeit zum Mindestlohn aufrechterhalten.

\section{Weitere Informationen}

OECD (2007), Benefits and Wages: OECD Indicators, Paris.

OECD (2008), Mehr Ungleichheit trotz Wachstum? Einkommensverteilung und Armut in OECD-Ländern, Paris.

\section{Anmerkungen zu den Abbildungen}

Abbildung EQ4.1: Italien und die Türkei haben keine allgemein geltende Mindestsicherung für Alleinerziehende. Die schattierte Fläche stellt den Bereich zwischen den verschiedenen Armutsschwellen von $40 \%, 50 \%$ und $60 \%$ des Haushaltsmedianeinkommens dar. In den Nettoeinkommen enthalten sind auch wohnungsbezogene Leistungen. Die Zahlenangaben unter den Balken beziehen sich auf den Prozentsatz von 2005.

Abbildung EQ4.2: Die Länder, die in EQ4.1, aber nicht in EQ4.2 aufgeführt sind, haben keinen gesetzlichen Mindestlohn. In den Nettoeinkommen sind auch wohnungsbezogene Leistungen enthalten. Die Zahlenangaben unter den Balken beziehen sich auf den Prozentsatz von 2005. 
EQ4.1. Ein-Eltern-Familien ohne Markteinkommen sind in einigen Ländern in hohem Maße armutsgefährdet Nettoeinkommen allein aus Sozialhilfeleistungen für Alleinerziehende mit zwei Kindern/Haushaltsmedianeinkommensäquivalent

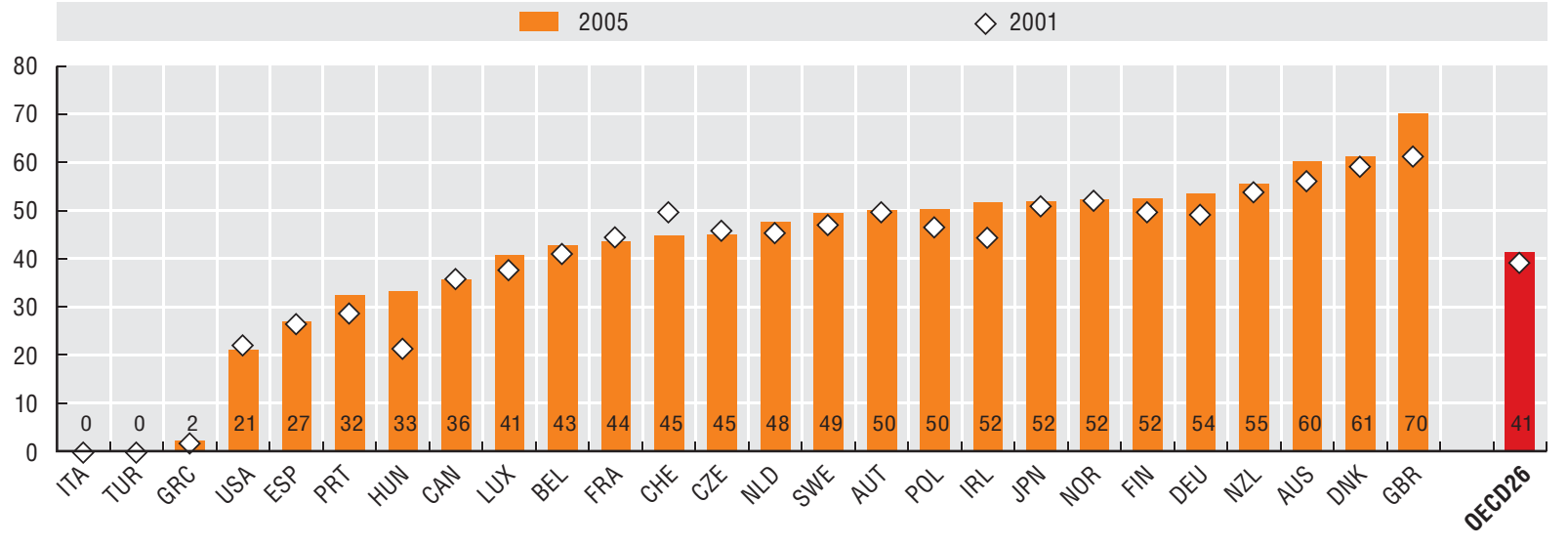

EQ4.2. Die Ausübung einer Vollzeitbeschäftigung kann Armut reduzieren, selbst wenn nur ein Mindestlohn bezogen wird

Nettoeinkommen für Alleinerziehende mit zwei Kindern bei Vollzeitbeschäftigung mit Mindestlohn/Haushaltsmedianeinkommensäquivalent

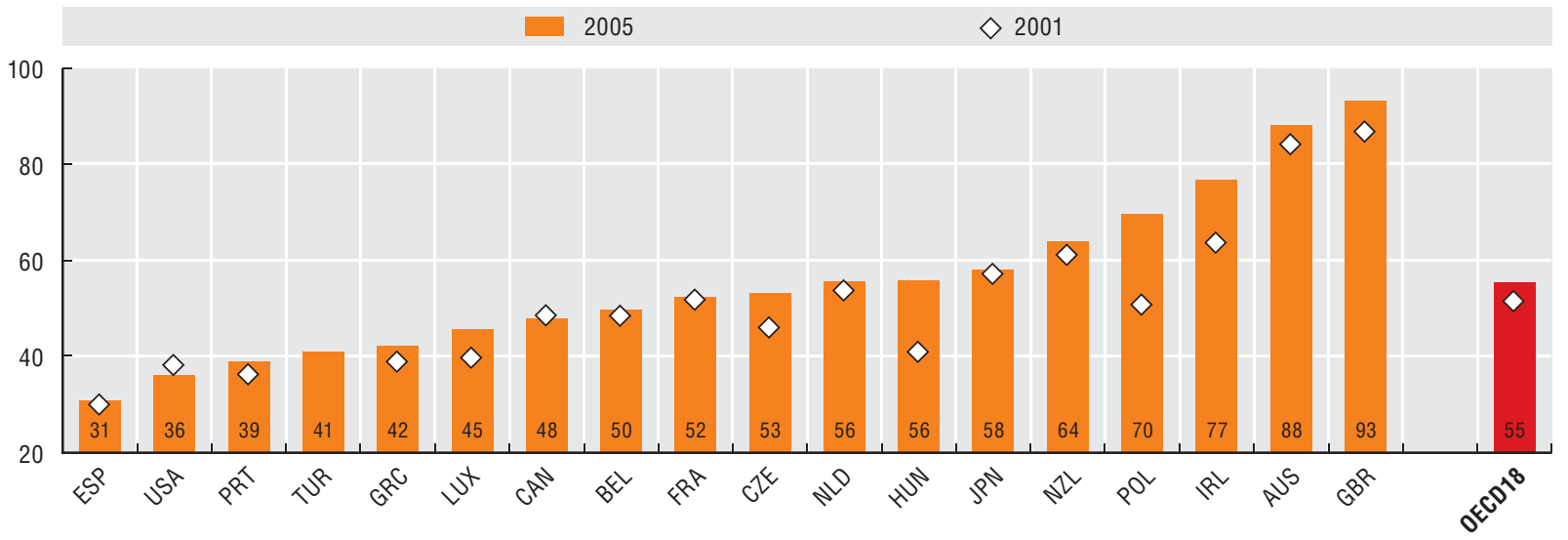

EQ4.3. Einigen Ländern gelingt es, stärkere Arbeitsanreize mit angemessenen Sicherheitsnetzen für Alleinerziehende zu verbinden

Prozentpunktdifferenz zwischen Vollzeitbeschäftigungseinkommen mit Mindestlohn und Mindestsicherungsleistungen im Verhältnis zum Medianeinkommen, im Vergleich zu Mindestsicherungsleistungen im Verhältnis zum Medianeinkommen, Alleinerziehende mit zwei Kindern

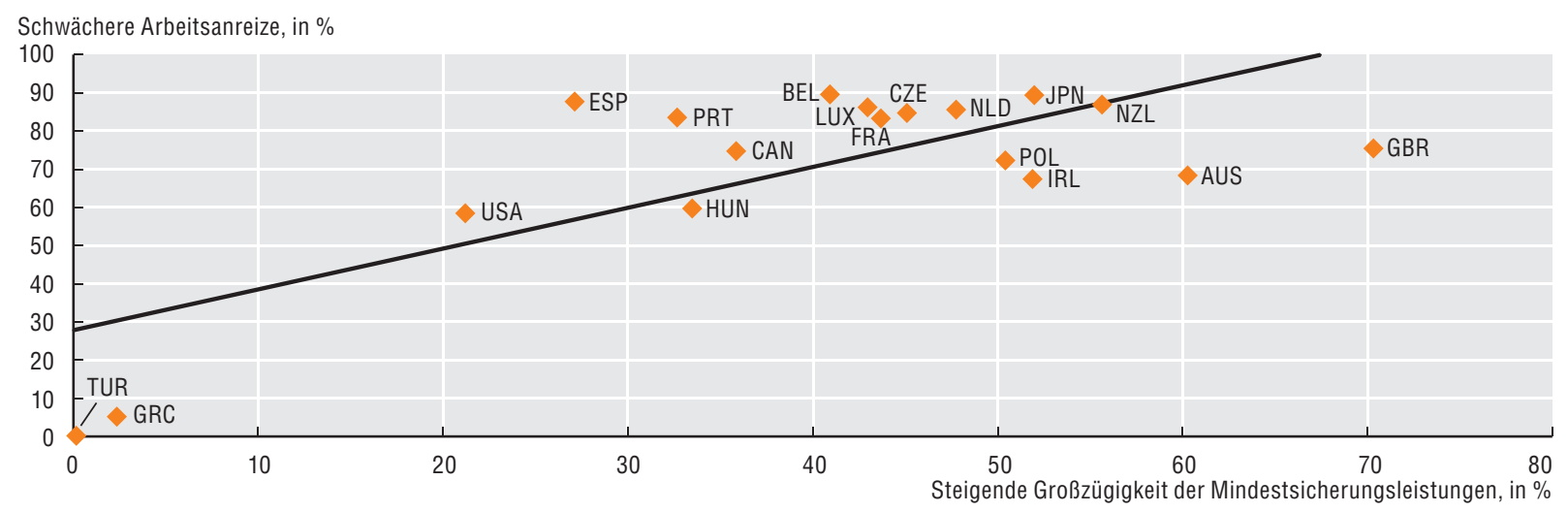




\section{Definition und Messung}

Ein breites Spektrum von Personen und Institutionen unterstützt auf verschiedenste Weise bedürftige Personen oder Haushalte. Ein Großteil dieser Unterstützung wird in Form von Sozialausgaben geleistet, bei denen es sich sowohl um finanzielle Beihilfen (durch Geldleistungen und Steuervorteile) als auch um Sachleistungen in Form von Gütern und Dienstleistungen handeln kann. Um zu den Sozialausgaben gerechnet zu werden, müssen diese Leistungen auf eine oder mehrere Notlagen ausgerichtet sein, wie Einkommensschwäche, Alter, Arbeitslosigkeit oder Behinderung.

Sozialausgaben gelten als öffentlich, wenn die entsprechenden Finanzströme vom Staat (d.h. von einer Zentralregierung, den nachgeordneten Gebietskörperschaften oder den Sozialversicherungsträgern) kontrolliert werden. Krankengeld ist beispielsweise eine „öffentliche“ Sozialleistung, wenn es aus Pflichtbeiträgen der Arbeitgeber und Arbeitnehmer zur Sozialversicherung finanziert wird, und eine „private“ Leistung, wenn es direkt vom Arbeitgeber an den Arbeitnehmer gezahlt wird. Der hier für die Zwecke des Ländervergleichs verwendete Indikator für die Sozialausgaben bezieht sich auf die öffentlichen Ausgaben im Verhältnis zum Nettonationaleinkommen. Die hier dargestellten Ausgabenströme sind „brutto" angegeben, d.h. vor Abzug von direkten und indirekten, auf diese Leistungen zu entrichtenden Steuern und vor Addition von Steuererleichterungen für soziale Zwecke („Bruttoausgaben“). Die Ausgaben der nachgeordneten Gebietskörperschaften sind in einigen Ländern, insbesondere Ländern mit stärker dezentralisierten oder föderalen Staatsverwaltungsformen, möglicherweise unterschätzt.

2005 machten die öffentlichen Bruttosozialausgaben im Durchschnitt von 30 OECD-Ländern $24 \%$ des NNE aus (Abbildung EQ5.1). Bei der Höhe der Ausgaben bestehen große Unterschiede zwischen den Ländern. In Mexiko und Korea sind es $8 \%$ des NNE, in Schweden dagegen 26 Prozentpunkte mehr. Dicht hinter Schweden liegen mit ihren Ausgaben Frankreich, Luxemburg und Österreich. Was Geldleistungen betrifft, so wenden die drei letztgenannten Länder (und andere Länder) erheblich mehr Mittel auf als Schweden. Eine erhebliche Anzahl von Ländern bildet relativ dichte Cluster knapp über und - insbesondere - knapp unter dem OECD-Durchschnitt.
In 25 Ländern überstiegen die Geldleistungen die Sachleistungen. In Polen, Italien und Österreich überstiegen die Geldleistungen die Sachleistungen um mindestens 10 Prozentpunkte des NNE, wohingegen in Island, Kanada und Mexiko die Dienstleistungen dominieren und mindestens 3 Prozentpunkte des NNE über den Geldleistungen liegen.

Die drei größten Kategorien öffentlicher Sozialausgaben sind die Renten (durchschnittlich 9\% des NNE), die Gesundheitsausgaben (7\%) und die Einkommenstransfers für Personen im Erwerbsalter (5\%). Die Rentenausgaben betragen in Deutschland, Frankreich, Griechenland, Italien, Österreich und Polen mehr als 12\% des NNE, und in Australien, Irland, Island, Kanada, Korea und Mexiko weniger als $5 \%$. Die öffentlichen Bruttoausgaben für soziale Leistungen belaufen sich nur in Dänemark, Norwegen und Island auf mehr als 5\% des NNE.

Der NNE-Anteil der öffentlichen Ausgaben für soziale Leistungen hat sich in der letzten Generation erhöht und konvergiert in allen OECD-Ländern (Abbildung EQ5.2). Der Gesamtanstieg betrug durchschnittlich 5 Prozentpunkte. Der überwiegende Teil dieses Zuwachses entfällt auf den Zeitraum 1980-1992. In der Zeit danach ist ein Zeittrend weniger offensichtlich. Der linke Teil der Abbildung EQ5.2 zeigt die Entwicklungen in ausgewählten Ländern mit hohen Ausgaben, der rechte Teil in Ländern mit geringen Ausgaben. Die Stabilisierung der Ausgabenanteile nach 1992 scheint in stärkerem Maße durch die Länder mit hohen Ausgaben zustande gekommen zu sein, wobei die Ausgaben nach 1992, insbesondere in Schweden, erheblich zurückgegangen sind. Die Länder mit geringeren Ausgaben weisen dagegen über den ganzen Zeitraum einen stetigeren Aufwärtstrend auf. Aus diesem Muster ergibt sich letztlich eine zunehmende Konvergenz der Sozialausgabenmuster in den OECD-Ländern, zumindest was den NNE-Anteil betrifft.

\section{Anmerkungen zu den Abbildungen}

Abbildung EQ5.1: Die Länder sind in absteigender Reihenfolge nach der Höhe der öffentlichen Sozialausgaben im Verhältnis zum NNE dargestellt. Ausgaben für aktive Arbeitsmarktprogramme (AAMP) können nicht in Geld- und Sachleistungen aufgeschlüsselt werden; in den öffentlichen Gesamtausgaben sind sie allerdings berücksichtigt (Werte in Klammern). Für Portugal beziehen sich die Daten auf 2004.

Abbildung EQ5.2: Für 23 Länder liegen Daten für den Zeitraum 1980-2005 vor, für Island, Korea, Mexiko, Polen und die Tschechische Republik liegen jedoch nur Daten ab 1990 vor. OECD30 bezieht sich auf einen ungewichteten Durchschnittswert der OECD-Länder ohne Ungarn (Daten ab 1999) und die Slowakische Republik (Daten ab 1995). Die 2005-Daten für Portugal beziehen sich auf 2004. 
EQ5.1. Die öffentlichen Sozialausgaben machen im OECD-Durchschnitt ein Viertel des NNE aus Öffentliche Bruttosozialausgaben nach Kategorie, in Prozent des NNE, 2005

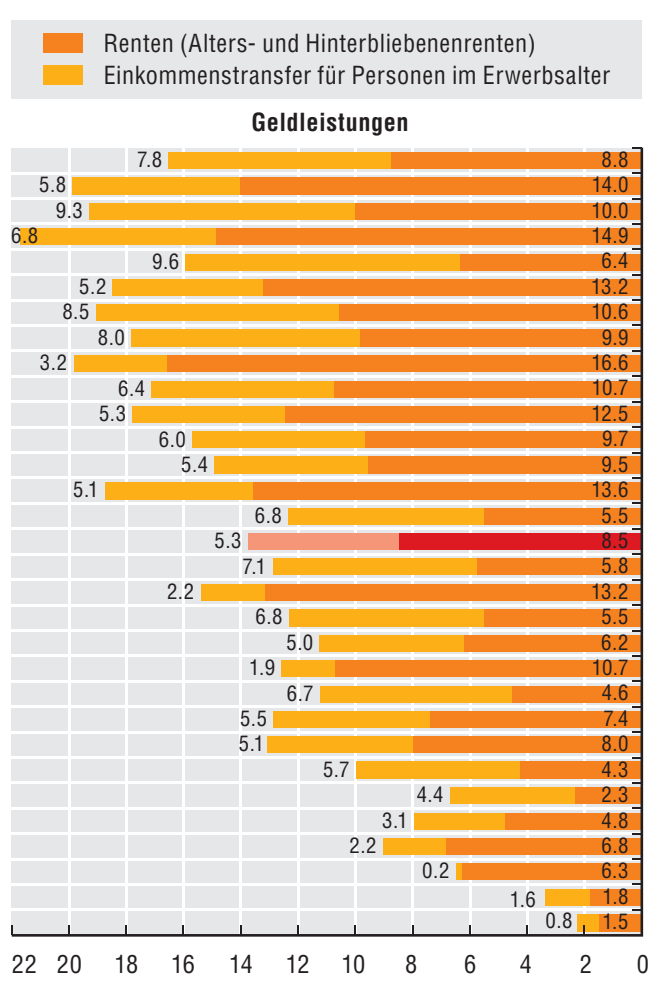

Schweden (33.6) Frankreich (33.2) Luxemburg (32.2)

Österreich (32.1)

Dänemark (31.5)

Deutschland (31.1) Belgien (31)

Finnland (30.5)

Italien (29.7)

Ungarn (28.4)

Portugal (28.2)

Spanien (25.5)

Tschech. Rep. (25.5)

Polen (25.1)

Norwegen (24.6)

OECD30 (24.4)

Niederlande (24.3)

Griechenland (23.6)

Neuseeland (23.5)

Ver. Königreich (23.3) Japan $(22.9)$

Irland (22.5)

Schweiz (22.2)

Slowak. Rep. (21.3)

Australien (21.2)

Island (19.9)

Kanada (19.3)

Ver. Staaten (18.1)

Türkei (11)

Korea $(8.0)$

Mexiko (7.9)

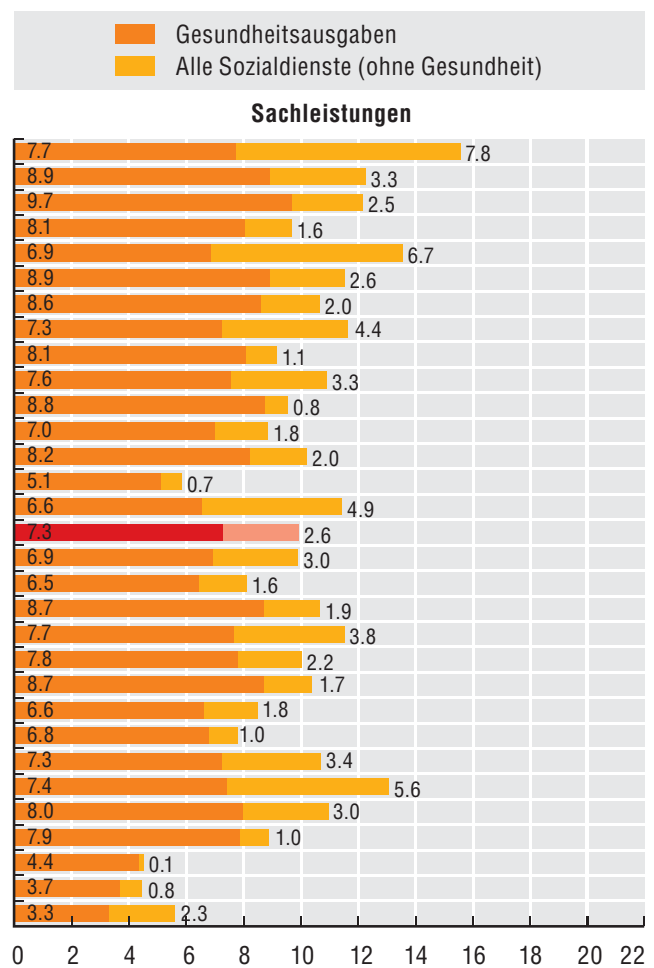

EQ5.2. Aufwärtstrend der öffentlichen Sozialausgaben im Verhältnis zum NNE

Öffentliche Bruttosozialausgaben in ausgewählten OECD-Ländern, in Prozent des NNE, 1980-2005
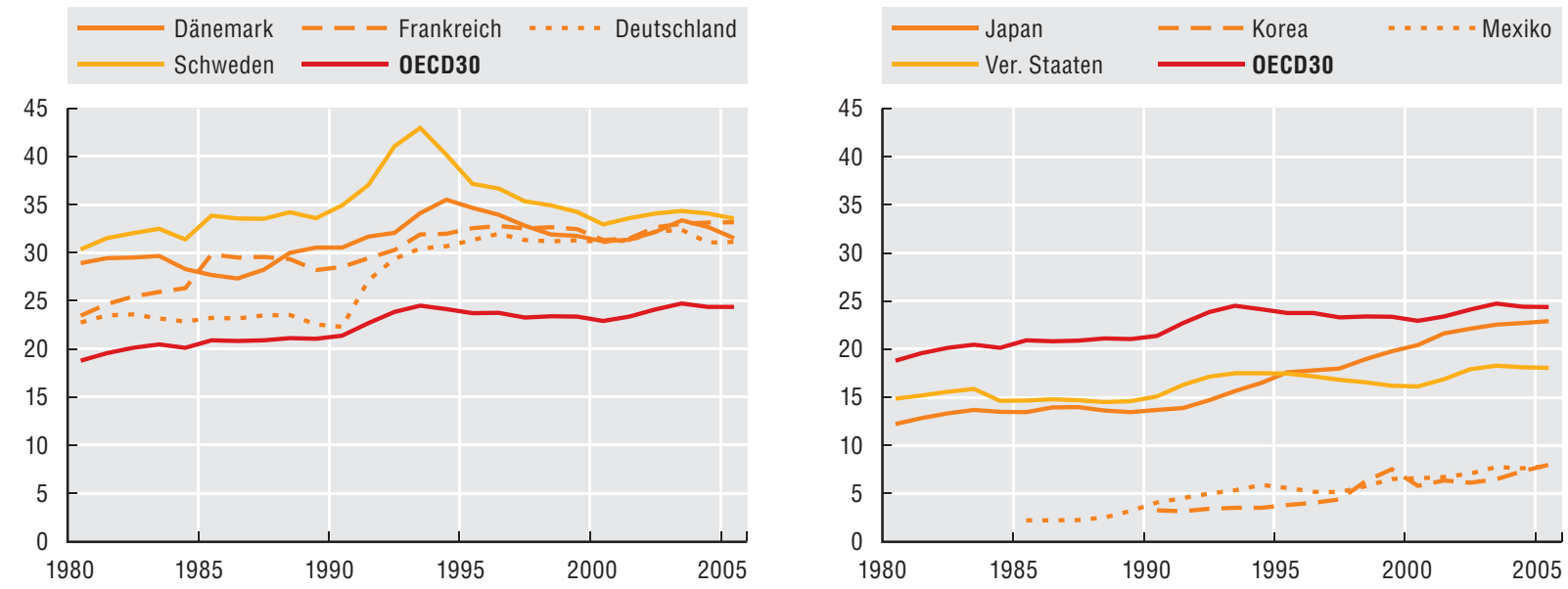

Quelle: OECD(2008), Social Expenditure database, 1980-2005 (www.oecd.org/els/social/expenditure). 


\section{Definition und Messung}

Für eine umfassende Beurteilung der Gesamthöhe der Ressourcen, die von den OECD-Ländern für soziale Zwecke aufgewendet werden, gilt es sowohl die öffentlichen als auch die privaten Sozialausgaben $\mathrm{zu}$ berücksichtigen und $\mathrm{zu}$ untersuchen, inwieweit das Steuersystem die tatsächliche Höhe der geleisteten Unterstützung beeinflusst. Zur Erfassung des Effekts des Steuersystems auf die Bruttosozialausgaben (d.h. vor Steuern) sollte der Teil der Sozialausgaben berücksichtigt werden, der durch die direkte Besteuerung der Transfereinkommen und die indirekte Besteuerung der von den Leistungsempfängern konsumierten Güter und Dienstleistungen wieder an den Staat zurückfließt. Zudem kann der Staat soziale Ziele auch dadurch fördern, dass er sozial motivierte Steuervorteile gewährt (z.B. Steuerfreibeträge für Kinder), was in der Regel zu Gesamtsozialausgaben führt, die die Bruttoausgaben übersteigen. Aus Sicht der Gesellschaft sind die Nettosozialausgaben (d.h. nach Steuern) öffentlichen und privaten Ursprungs ein besserer Indikator für den Umfang der für soziale Zwecke aufgewendeten Mittel.

Da es oft an entsprechenden Verwaltungsdaten fehlt, sind zur Messung des Effekts des Steuersystems auf die Sozialausgaben häufig Schätzungen auf der Grundlage von Mikrodaten und Mikrosimulationsmodellen erforderlich. Zudem liegen nicht immer zentral erfasste Daten zu den privaten Sozialausgaben vor. Daher sind die entsprechenden Informationen vielleicht stärker mit Fehlern behaftet als die Daten zu den öffentlichen Bruttosozialausgaben (vgl. EQ5).

Im OECD-Durchschnitt flossen 2005 13\% der Bruttosozialausgaben über die Nettobesteuerung an den Staat zurück (Tabelle EQ6.1). Der über die Nettobesteuerung an den Staat zurückfließende Betrag ist jedoch unterschiedlich, so dass die Länderrangfolge anhand der Bruttoausgaben eine ganz andere sein könnte als anhand der Nettoausgaben. Bei der Untersuchung der Muster der Auswirkungen von Steuerzahlungen und Steuergutschriften auf die Bruttosozialausgaben des Staats in ausgewählten OECD-Ländern fallen drei Dinge auf. Erstens ist der Rückfluss der Bruttosozialausgaben in Form direkter, auf Transfereinkommen zu entrichtender Steuern in den nordischen Sozialstaaten Dänemark, Finnland und Schweden, wo zwischen einem
Fünftel und einem Viertel der Bruttoausgaben wieder in die staatlichen Kassen zurückfließen, am höchsten. Zweitens sind die öffentlichen Nettosozialausgaben in Mexiko, den Vereinigten Staaten und Korea höher als die Bruttoausgaben (in Kanada sind sie wertmäßig gleich hoch). Tatsächlich ist der absolute Betrag, um den die öffentlichen Nettoausgaben in Mexiko die Bruttosozialausgaben übersteigen, proportional mit dem Betrag vergleichbar, um den die Nettoausgaben in den drei nordischen Ländern unter den Bruttoausgaben liegen.

Im Durchschnitt einer Gruppe von 26 OECD-Ländern machten die öffentlichen Nettosozialausgaben 2003 25\% des NNE aus. Der Anteil reichte von 9\% in Korea bis 33\% in Deutschland und 35\% in Frankreich. Länder mit geringen Bruttosozialausgaben gewähren mehr Steuervergünstigungen im unteren Bereich der Einkommensverteilung, und in den nordischen Ländern mit ihren hohen Bruttoausgaben fließt ein erheblicher Anteil wieder über die Steuern in die Staatskasse zurück. Bei Berücksichtigung sowohl der privaten Sozialleistungen als auch des Effekts des Steuersystems verringern sich die Unterschiede, die in Bezug auf die Höhe der Sozialausgaben im Verhältnis zum NNE zwischen den Ländern zu beobachten sind, erheblich. Eine im Ländervergleich ähnlichere Höhe der Nettosozialausgaben bedeutet jedoch nicht, dass der Grad der vom Steuer- und Transfersystem gewährleisteten Umverteilung ebenfalls identisch wäre, da es sich hier nur um ungewichtete Länderdurchschnitte handelt, die keinen Aufschluss darüber geben, wer wie viel erhält.

Die Gesamtnettosozialausgaben belaufen sich in den OECD-Ländern (einschließlich Privatausgaben) auf $28 \%$ des NNE (Abbildung EQ6.2). Durch die Einbeziehung der Privatausgaben kommen durchschnittlich 3 Prozentpunkte des NNE hinzu. Das Land, das durch die Einbeziehung der Privatausgaben im Länderranking den größten Sprung nach oben macht, sind die Vereinigten Staaten, die bei den öffentlichen Sozialausgaben an drittletzter Stelle rangieren, aber mit 31\% des NNE über den OECD-Durchschnitt hinauskommen. Der Grund hierfür sind die hinzuzurechnenden 10 Prozentpunkte der Privatausgaben.

\section{Anmerkungen zu den Abbildungen und Tabellen}

Tabelle EQ6.1: - Null; .. Daten nicht verfügbar.

Abbildung EQ6.2: Die Länder sind in absteigender Reihenfolge nach der Höhe der gesamten Nettosozialausgaben dargestellt; da eine Berichtigung um indirekte Steuern vorgenommen werden muss, werden die Nettosozialausgaben im Verhältnis zum NNE zu Faktorkosten und nicht zu Marktpreisen gemessen. 
EQ6.1. Öffentliche Brutto- und Nettosozialausgaben

In Prozent des NNE zu Faktorkosten, 2005

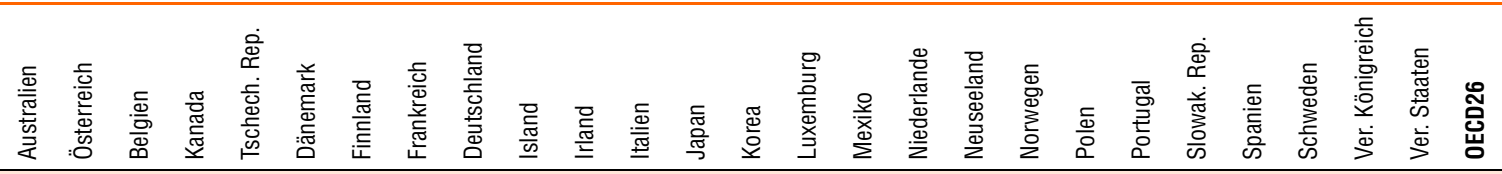

Öffentliche Bruttosozialausgaben

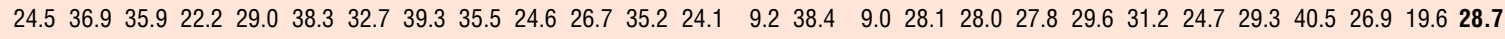

- Auf Transfereinkommen zu entrichtende direkte Steuern und Sozialversicherungsbeiträge

$\begin{array}{lllllllllllllllllllllllllll}0.3 & 3.3 & 1.9 & 0.5 & 0.0 & 5.7 & 3.5 & 1.9 & 1.8 & 1.0 & 0.3 & 2.7 & 0.3 & 0.0 & 1.3 & - & 2.1 & 1.9 & 2.3 & 2.2 & 0.9 & - & 1.6 & 5.5 & 0.3 & 0.6 & \mathbf{1 . 7}\end{array}$

- Indirekte Besteuerung der von Leistungsempfängern konsumierten Güter und Dienstleistungen

$\begin{array}{lllllllllllllllllllllllllll}1.1 & 3.6 & 3.2 & 1.0 & 3.0 & 3.9 & 3.6 & 3.3 & 2.6 & 1.6 & 2.9 & 2.6 & 0.8 & 0.5 & 4.9 & 0.2 & 2.4 & 2.0 & 2.7 & 3.4 & 3.6 & 2.6 & 2.2 & 3.2 & 1.7 & 0.4 & 2.4\end{array}$

+ Steuervergünstigungen für Empfänger von Transferleistungen (ohne Renten)

$\begin{array}{lllllllllllllllllllllllllll}0.5 & 0.1 & 0.6 & 1.5 & 0.7 & 0.0 & - & 1.3 & 2.3 & - & 0.6 & 0.3 & 1.0 & 0.7 & 0.0 & 1.8 & 1.0 & 0.1 & 0.1 & 0.1 & 1.3 & 0.1 & 0.6 & - & 0.5 & 2.5 & \mathbf{0} .8\end{array}$

$=$ Öffentliche Nettosozialausgaben

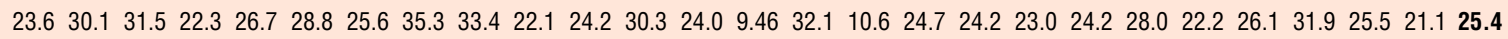
Nachrichtlich

Steuervergünstigungen für Rentner

$\begin{array}{lllllllllllllllllllllllllllll}2.8 & 0.1 & 0.2 & 2.3 & 0.2 & . . & 0.2 & 0.0 & 1.2 & 1.5 & 2.2 & 0.0 & 0.1 & . & . . & 0.1 & . . & . . & 0.8 & 0.3 & 0.1 & 0.2 & 0.4 & 0.0 & 1.6 & 1.0 & \ldots\end{array}$

\section{EQ6.2. Öffentliche Sozialausgaben und Gesamtsozialausgaben}

In Prozent des NNE zu Faktorkosten, 2005

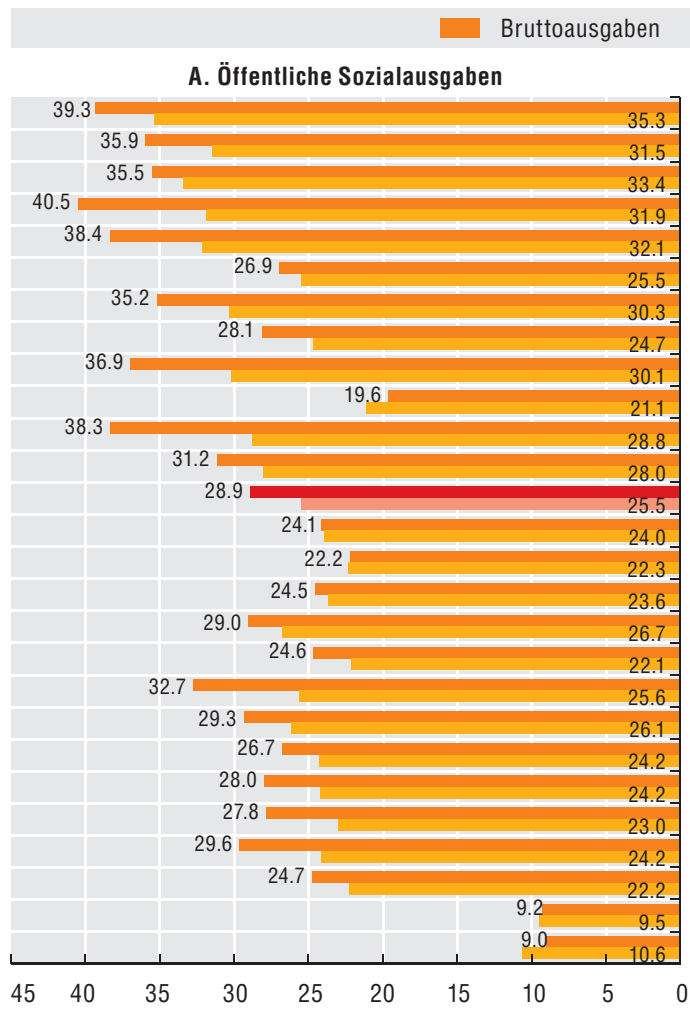

Frankreich
Belgien
Deutschland
Schweden
Luxemburg
Ver. Königreich
Italien
Niederlande
Österreich
Dänemark
Ver. Staaten
Portugal
OECD26
Japan
Kanada
Australien
Tschech. Rep.
Island
Finnland
Spanien
Irland
Neuseeland
Norwegen
Polen
Slowak. Rep.
Korea
Mexiko

\section{Nettoausgaben}

\section{B. Gesamte (öffentliche + private) Sozialausgaben}

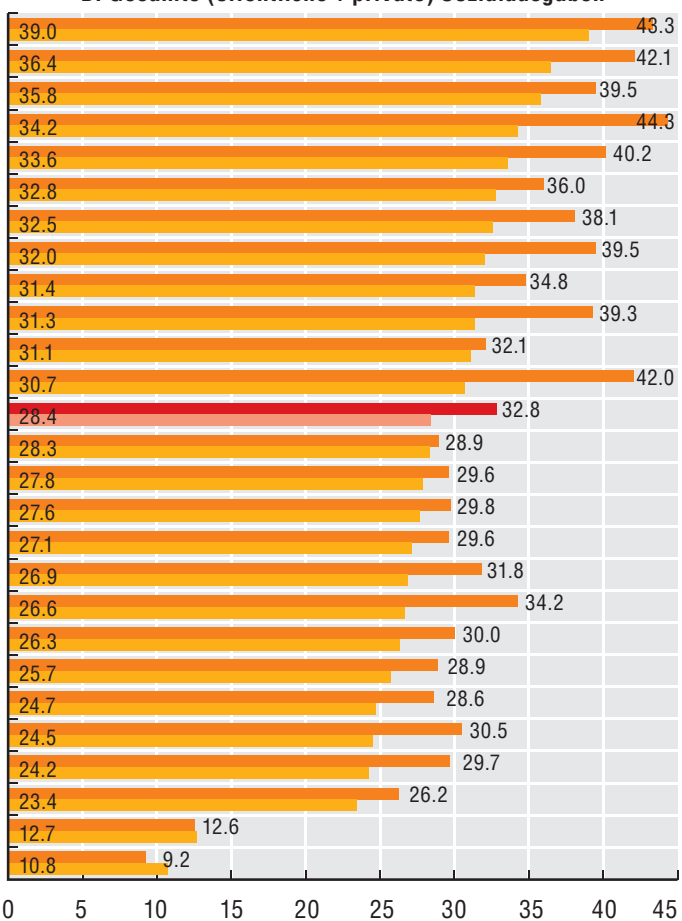

Quelle: OECD (2008), Social Expenditure Database 1980-2005 (www.oecd.org/els/social/expenditure). 


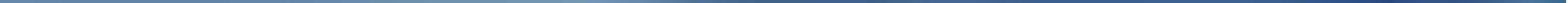




\section{GESUNDHEITSINDIKATOREN}

1. Lebenserwartung

2. Selbsteinschätzung des Gesundheitszustands

3. Gesundheitszustand von Säuglingen

4. Fettleibigkeit

5. Körpergröße

6. Seelische Gesundheit

7. Langzeitpflegebedürftige

8. Gesundheitsausgaben 


\section{Definition und Messung}

Die Lebenserwartung ist der allgemeinste und bekannteste Indikator des Gesundheitszustands der Bevölkerung. Sie ist definiert als die durchschnittliche Zahl der einer Person voraussichtlich verbleibenden Lebensjahre gemäß der altersspezifischen Mortalitätsrate in einem gegebenen Land und in einem bestimmten Jahr. Der Effekt eines künftigen Rückgangs der altersspezifischen Sterberaten ist darin nicht berücksichtigt. Jedes Land verwendet etwas andere Methoden zur Berechnung der Lebenserwartung. Diese unterschiedlichen Methoden können die Vergleichbarkeit der übermittelten Schätzungen beeinträchtigen, da sie den Indikator der Lebenserwartung eines Landes geringfügig verändern können.

Die Lebenserwartung bei der Geburt ist in den OECDLändern erheblich gestiegen. Zwischen 1960 und 2006 hat die durchschnittliche Lebenserwartung bei der Geburt im OECD-Raum für Frauen um 10,9 Jahre auf 81,7 Jahre zugenommen. Die Lebenserwartung der Männer erhöhte sich um 10,2 Jahre auf 76,0 Jahre (HE1.1). 2006 war die Lebenserwartung bei der Geburt für Frauen in Japan am höchsten (85,8 Jahre), gefolgt von Frankreich, Spanien, der Schweiz und Italien. Bei den Männern wies Island die höchste Lebenserwartung auf (79,4 Jahre), gefolgt von der Schweiz, Japan, Australien und Schweden.

Der Anstieg der Lebenserwartung bei der Geburt im OECD-Raum ging mit einer starken Verringerung der Unterschiede zwischen den einzelnen Ländern einher. In Korea und der Türkei erhöhte sich die Lebenserwartung bei der Geburt im Zeitraum 1960-2006 für Frauen und Männer zusammengenommen um 26,7 bzw. 23,3 Jahre, während sie in Mexiko um mehr als 18 Jahre stieg. Das Aufschließen dieser Länder in Bezug auf die Lebenserwartung erklärte sich hauptsächlich aus einer deutlich zunehmenden Konvergenz der Säuglingssterblichkeitsraten.

Es gibt kaum Anzeichen dafür, dass sich der Anstieg der Lebenserwartung einem Plafond nähert. Die Zuwachsrate der Lebenserwartung bei der Geburt ging für japanische Frauen nach der Aufholphase auf die Hälfte zurück, seither setzte sich der Anstieg aber mit rd. 3\% jährlich fort.
Die Differenz bei der Lebenserwartung von Männern und Frauen hat sich etwas vergrößert. Da die Lebenserwartung bei der Geburt für Frauen seit 1960 stärker gestiegen ist als für Männer, hat sich die Geschlechterdifferenz bei der Lebenserwartung im Zeitraum 1960-2006 im Durchschnitt von 5,0 Jahren auf 5,7 Jahre ausgeweitet. Dabei verlief der Trend im ersten und im zweiten Teil dieses Zeitraums allerdings unterschiedlich. Während der Geschlechterunterschied bei der Lebenserwartung in den 1960er und 1970er Jahren erheblich zunahm (auf einen Höchststand von durchschnittlich 6,7 Jahren im Jahr 1980), hat er sich in den letzten 25 Jahren verkleinert. Diese Verkleinerung ist z.T. auf die zunehmende Konvergenz im Risikoverhalten von Männern und Frauen (z.B. in Bezug auf das Rauchen) und die niedrigeren Mortalitätsraten bei Herz-Kreislauf-Erkrankungen unter Männern zurückzuführen.

Ältere Menschen leben länger. Auch die Lebenserwartung älterer Menschen hat sich dank eines besseren Zugangs zu Gesundheitsdiensten und des medizinischen Fortschritts, vor allem was die Behandlung von Herz-Kreislauf-Erkrankungen betrifft, in den letzten Jahrzehnten wesentlich erhöht. Im Jahr 2006 lag die Restlebenserwartung von Frauen im Alter von 65 Jahren durchschnittlich bei 20,1 Jahren und war damit um 5,3 Jahre höher als 1960. Männer im selben Alter hatten eine Restlebenserwartung von 16,7 Jahren, d.h. 4,0 Jahre mehr als 1960 (Abbildung HE1.2). Der Geschlechterunterschied bei der Lebenserwartung in höherem Alter hat sich in mehreren OECDLändern seit Mitte der 1980er Jahre verkleinert, und dieser Trend wird sich den Projektionen zufolge künftig fortsetzen.

Die allgemeine Erhöhung der Lebenserwartung erklärt sich aus einem gestiegenen Lebensstandard, einer besseren Ernährung, einer Abnahme des Tabak- und Alkoholkonsums, einem höheren Bildungsniveau sowie einem leichteren Zugang zu guten Gesundheitsdiensten. In den unteren sozioökonomischen Schichten fiel der Anstieg der Lebenserwartung allerdings geringer aus.

\section{Weitere Informationen}

OECD (2004), Towards High-performing Health Systems, OECD, Paris.

\section{Anmerkungen zu den Abbildungen}

Abbildung HE1.2: 2005 für Kanada, das Vereinigte Königreich und die Vereinigten Staaten; 2004 für Italien. 


\section{GESUNDHEITSINDIKATOREN}

1. Lebenserwartung

HE1.1. Die Lebenserwartung bei der Geburt ist im OECD-Raum deutlich gestiegen

Lebenserwartung bei der Geburt, in Jahren, Männer und Frauen, 1960 und 2006

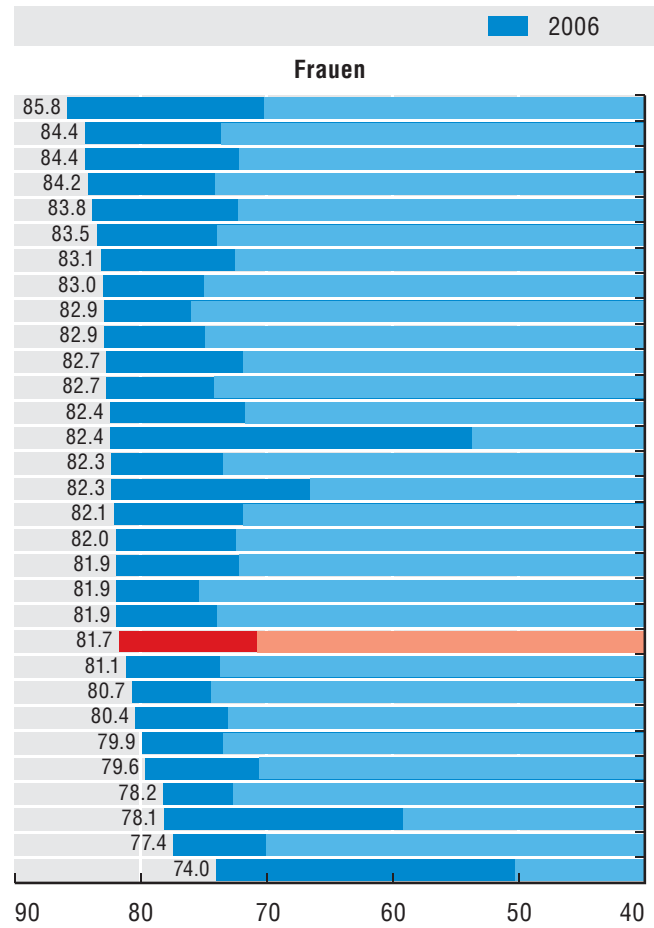

$$
\begin{gathered}
\text { Japan } \\
\text { Frankreich } \\
\text { Spanien } \\
\text { Schweiz } \\
\text { Italien } \\
\text { Australien } \\
\text { Finnland } \\
\text { Island } \\
\text { Norwegen } \\
\text { Schweden } \\
\text { Österreich } \\
\text { Kanada } \\
\text { Deutschland } \\
\text { Korea } \\
\text { Belgien } \\
\text { Portugal } \\
\text { Irland } \\
\text { Griechenland } \\
\text { Luxemburg } \\
\text { Niederlande } \\
\text { Neuseeland } \\
\text { OECD } \\
\text { Ver. Königreich } \\
\text { Dänemark } \\
\text { Ver. Staaten } \\
\text { Tschech. Rep. } \\
\text { Polen } \\
\text { Slowak. Rep. } \\
\text { Mexiko } \\
\text { Ungarn } \\
\text { Türkei }
\end{gathered}
$$
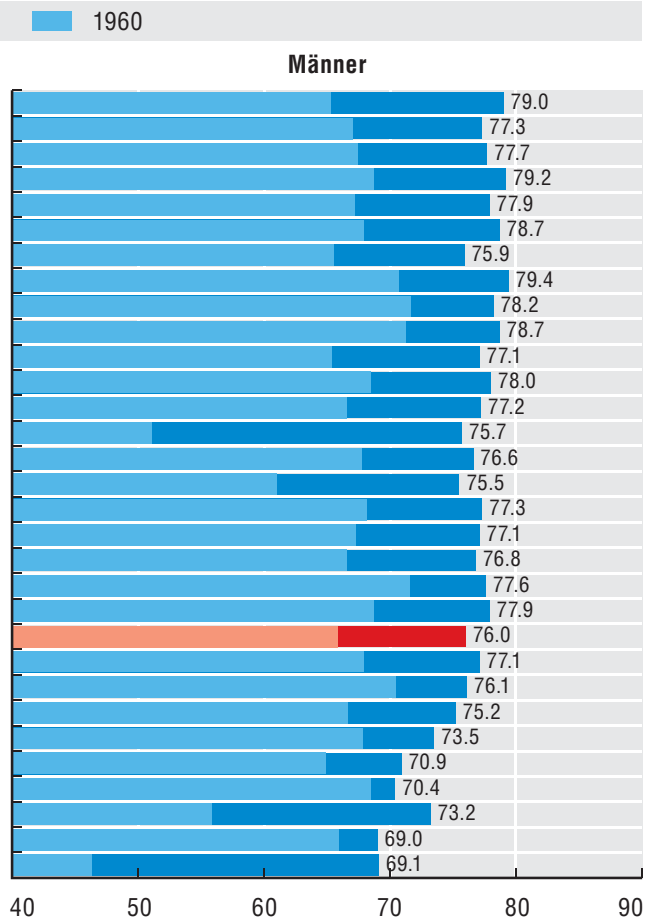

HE1.2. Ältere Menschen haben eine beachtliche Restlebenserwartung Lebenserwartung mit 65 Jahren, in Jahren, Männer und Frauen, 2006

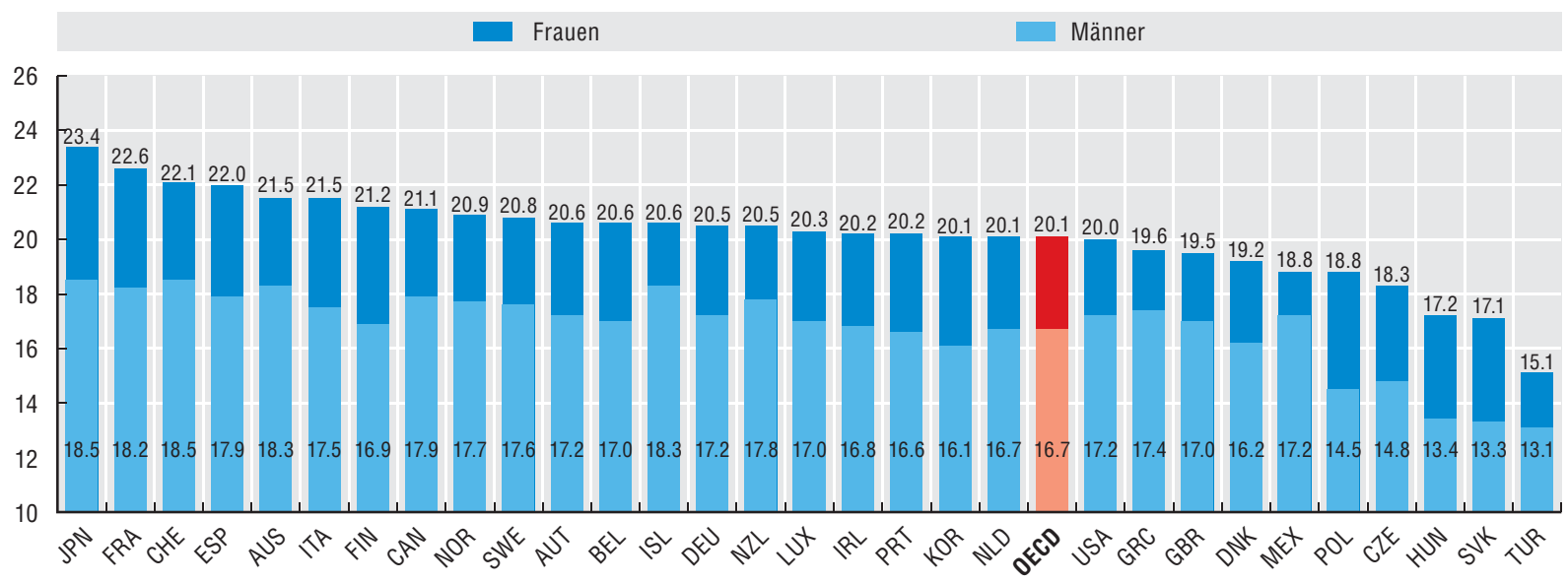

Quelle: OECD (2008), OECD-Gesundheitsdaten 2008, CD-Rom, OECD

Paris (www.oecd.org/health/healthdata). 


\section{Definition und Messung}

In den meisten OECD-Ländern werden regelmäßig Erhebungen zum Gesundheitszustand der Bevölkerung durchgeführt, in denen Fragen gestellt werden wie: „Wie gut ist Ihr allgemeiner Gesundheitszustand? Sehr gut, gut, mittelmäßig, schlecht, sehr schlecht“. Trotz der eher allgemein gehalten Formulierung und der Subjektivität dieser Fragen hat sich gezeigt, dass Indikatoren der Selbsteinschätzung des Gesundheitszustands ein guter Prädiktor für die künftige Inanspruchnahme von Gesundheitsversorgung und die künftigen Sterberaten sind (Miilunpalo et al., 1997).

Bei Ländervergleichen der Selbsteinschätzung des Gesundheitszustands ist aus zwei Gründen Vorsicht geboten. Erstens kann die Selbsteinschätzung des Gesundheitszustands durch eine Reihe von Faktoren beeinflusst werden, die über den „tatsächlichen“ Gesundheitszustand hinausgehen, wie z.B. der kulturelle Hintergrund. Zweitens unterscheiden sich die Fragen und die Antwortkategorien in den einzelnen Ländern bzw. Erhebungen. Insbesondere ist in den Vereinigten Staaten, Kanada, Neuseeland und Australien eine Verzerrung zu Gunsten einer positiven Gesundheitseinschätzung festzustellen, die sich aus den fünf Antwortkategorien „hervorragend, sehr gut, gut, mittelmäßig, schlecht“ erklärt. Die Angaben beziehen sich für diese Länder auf Personen, die eine der drei positiven Kategorien angekreuzt haben („hervorragend“, „sehr gut“, „gut“). In den meisten anderen OECD-Ländern ist die Antwortskala hingegen symmetrisch („sehr gut“, „gut“, „mittelmäBig“, "schlecht“, „sehr schlecht“). Die Angaben für diese Länder beziehen sich nur auf die ersten beiden Kategorien („sehr gut“ und „gut").

Die meisten Menschen betrachten ihren Gesundheitszustand als gut. In der Hälfte der OECD-Länder stufen mindestens $75 \%$ der Erwachsenenbevölkerung ihren Gesundheitszustand als gut, sehr gut oder hervorragend ein (HE2.1). Neuseeland, die Vereinigten Staaten und Kanada sind die drei Länder mit dem höchsten Prozentsatz an Personen, die ihren Gesundheitszustand als gut oder sehr gut einschätzen; ungefähr neun von zehn Befragten erfreuen sich dort laut eigenen Angaben einer guten Gesundheit. Bei diesen günstigen Ergebnissen könnte es sich um ein statistisches Artefakt handeln (vgl. „Definition und Messung“). In Mexiko, Spanien und Finnland betrachten etwa zwei Drittel der Erwachsenenbevölkerung ihren Gesundheits- zustand als gut oder sehr gut. Am unteren Ende der Skala stehen die Slowakische Republik, Japan, Portugal, Ungarn und Korea, wo weniger als die Hälfte der Erwachsenenbevölkerung ihren Gesundheitszustand als gut oder sehr gut einstuft.

Männer halten sich für gesünder als Frauen. In der Mehrzahl der Länder betrachten Männer im Vergleich zu Frauen ihren Gesundheitszustand mit größerer Wahrscheinlichkeit als gut oder besser (HE2.2). Erwartungsgemäß nimmt die positive Einschätzung des eigenen Gesundheitszustands mit zunehmendem Alter ab. In vielen Ländern ist eine besonders deutliche Abnahme des Anteils der Personen mit positiver Einschätzung des eigenen Gesundheitszustands ab dem Alter von 45 Jahren festzustellen, auf die ein weiterer deutlicher Rückgang im Alter von 65 Jahren folgt. In allen OECD-Ländern schätzen Personen mit niedrigerem Bildungs- oder Einkommensniveau ihren Gesundheitszustand weniger positiv ein als Menschen mit höherem Bildungs- oder Einkommensniveau.

Der Prozentsatz der Personen, die sich laut eigenen Angaben einer guten Gesundheit erfreuen, ist im Zeitverlauf stabil. In Ländern, für die lange Zeitreihen vorliegen, hat sich der Anteil der Erwachsenenbevölkerung, der seinen Gesundheitszustand als gut oder sehr gut einstuft, im Verlauf der letzten 25 Jahre nicht verändert (HE2.3). Dies gilt auch für die Bevölkerung ab 65 Jahre. Eine mögliche Erklärung für die weitgehend unveränderte Selbsteinschätzung des Gesundheitszustands im Verlauf der letzten 25 Jahre bei zugleich kontinuierlich steigender Lebenserwartung ist, dass die Menschen zwar länger leben, dabei aber nicht zwangsläufig gesünder sind. Eine andere mögliche Erklärung ist, dass sich die Sichtweise der Menschen ändert und dass sie angesichts eines sich insgesamt verbessernden Gesundheitszustands eine andere Vorstellung davon haben, was „Gesundsein“ bedeutet.

\section{Weitere Informationen}

Miilunpalo, S. et al. (1997), „Self-rated Health Status as a Health Measure: The Predictive Value of Selfreported Health Status on the Use of Physician Services and on Mortality in the Working-age Population“, Journal of Clinical Epidemiology, Vol. 50, No. 5, S. 517-528.

\section{Anmerkungen zu den Abbildungen}

Abbildungen HE2.1und HE2.2: Anmerkung 1: Die Ergebnisse dieser Länder sind nicht direkt vergleichbar mit denen anderer Länder, da methodologische Abweichungen im Erhebungsfragebogen zu einer Verzerrung nach oben führen. 


\section{GESUNDHEITSINDIKATOREN}

\section{Selbsteinschätzung des Gesundheitszustands}

\section{HE2.1. Die meisten Menschen betrachten sich als gesund}

Prozentsatz der Bevölkerung ab 15 Jahre, der sich laut eigenen Angaben einer guten Gesundheit erfreut

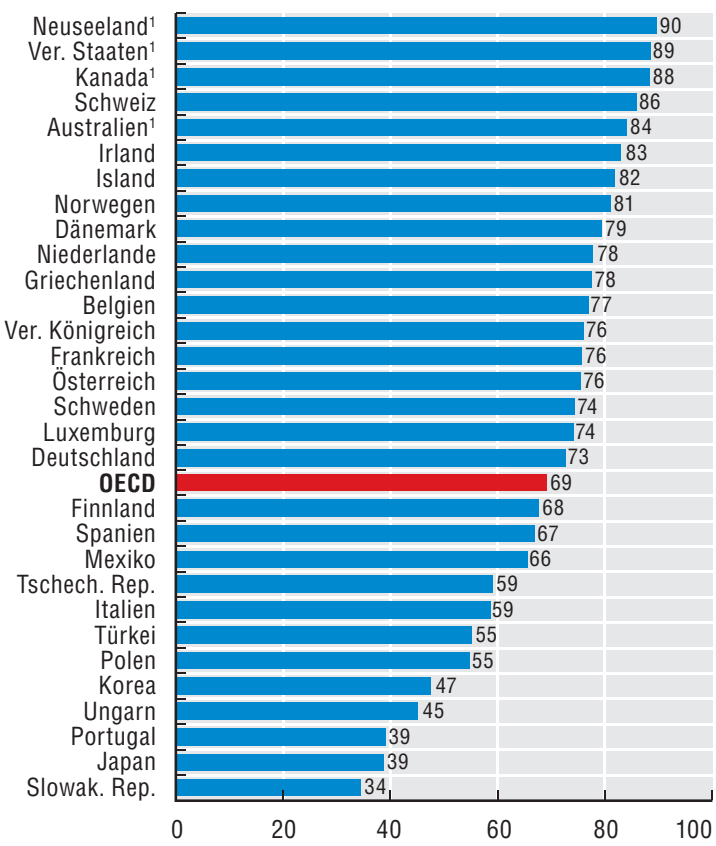

HE2.2. Männer halten ihren Gesundheitszustand für besser als Frauen

Differenz Frauen/Männer bei der Selbsteinschätzung des Gesundheitszustands, 2006 (oder letztes verfügbares Jahr)

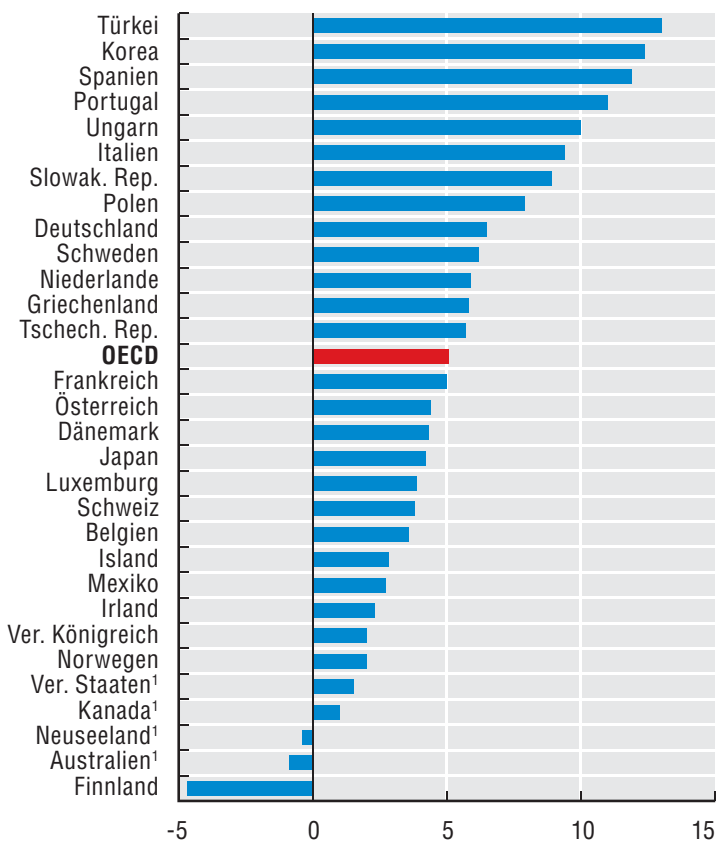

\section{HE2.3. Der Anteil der Personen mit positiver Einschätzung des eigenen Gesundheitszustands ist über lange Zeiträume stabil}

Prozentsatz der Bevölkerung ab 15 Jahre, der sich laut eigenen Angaben einer guten Gesundheit erfreut

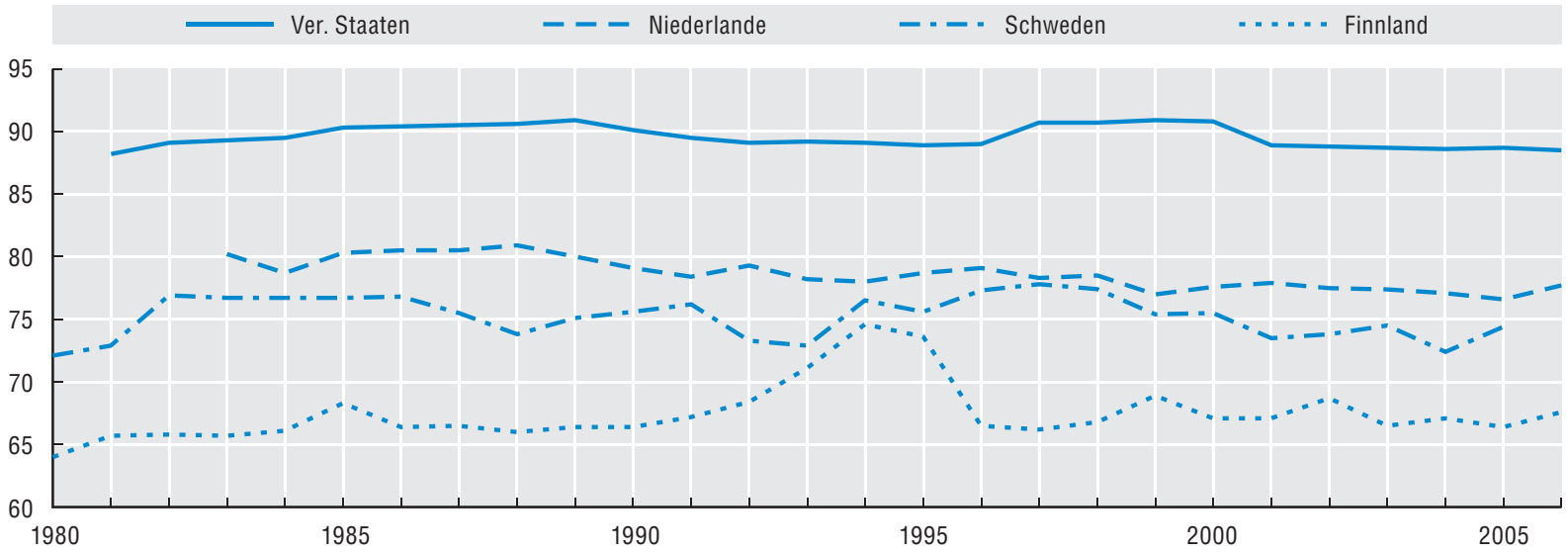

Quelle: OECD (2008), OECD-Gesundheitsdaten 2008, CD-Rom, OECD, 


\section{Definition und Messung}

Von einem niedrigen Geburtsgewicht wird laut der Definition der Weltgesundheitsorganisation (WHO) gesprochen, wenn das Gewicht eines Säuglings bei der Geburt, unabhängig von der Dauer der Schwangerschaft, unter 2500 Gramm liegt. Dieser Grenzwert basiert auf epidemiologischen Untersuchungen zum erhöhten Sterberisiko bei Säuglingen. Die Zahl der Neugeborenen mit niedrigem Gewicht wird in Prozent der Lebendgeburten ausgedrückt. Die Mehrzahl der Daten ist Geburtsregistern entnommen; die Daten für die Niederlande und die Türkei stammen allerdings aus nationalen Gesundheitserhebungen.

Die Säuglingssterberate ist definiert als die Zahl der jährlichen Todesfälle bei Kindern unter einem Lebensjahr je 1000 Lebendgeburten. Ein Teil der im internationalen Vergleich festzustellenden Unterschiede bei den Säuglingssterberaten ist u.U. darauf zurückzuführen, dass zwischen den Ländern Differenzen in Bezug auf die Definition der Lebendgeburten bestehen. In Belgien, Dänemark, Deutschland, Finnland, Italien, Österreich, Portugal, der Slowakischen Republik, Spanien, Schweden, Ungarn und dem Vereinigten Königreich gelten keine Einschränkungen je nach Dauer der Schwangerschaft oder Gewicht für die Erfassung von Totgeburten (EUROPERISTAT Project 2008). Auch in Kanada und den Vereinigten Staaten sind keine solchen Beschränkungen vorgesehen. Eine geringe Einschränkung gilt in Norwegen (für die Erfassung als Totgeburt muss die Schwangerschaftsdauer über 12 Wochen betragen haben), während in Frankreich, den Niederlanden, Polen und der Tschechischen Republik eine Mindestschwangerschaftsdauer von 22 Wochen und/oder ein Mindestgewicht von 500 Gramm vorausgesetzt wird. In Australien und Neuseeland sind keine Grenzwerte in Bezug auf die Schwangerschaftsdauer vorgesehen. Für den OECD-Raum insgesamt lassen sich die Regelungen nicht klar definieren, sie dürften zwischen den einzelnen Ländern jedoch nicht stark abweichen.

Das niedrige Geburtsgewicht und die Säuglingssterblichkeit sind wichtige Indikatoren der Säuglingsgesundheit. Bei Säuglingen mit niedrigem Geburtsgewicht ist die Gefahr von Gesundheitsschäden und Entwicklungsschwierigkeiten größer. Zu den Risikofaktoren für ein niedriges Geburtsgewicht sowie für Todesfälle im Säuglingsalter gehören der sozioökonomische Status der Eltern, das Alter der Mutter, Mehrlingsgeburten, Tabak- und Alkoholkonsum der Mutter sowie die Rechtzeitigkeit des Zugangs zu pränataler Versorgung und die Qualität dieser Dienste.

Im OECD-Durchschnitt handelt es sich bei jedem 15. Neugeborenen um einen Fall von niedrigem Geburtsgewicht. Innerhalb der Gruppe der OECD-Länder ist der Anteil der Neugeborenen mit niedrigem Geburtsgewicht mit 4,5\% in Island, Schweden, Finnland und Korea am geringsten. Am anderen Ende der Skala liegen die Türkei, Japan und Griechenland mit einem Anteil von über 9\% (HE3.1). Mexiko,
Ungarn und die Vereinigten Staaten folgen dicht dahinter mit einem Anteil von über $8 \%$ der Lebendgeburten.

Im OECD-Durchschnitt stirbt eines von 200 Kindern im Säuglingsalter. Die Variationsbreite der Säuglingssterblichkeit im OECD-Raum reichte 2006 von weniger als 3 Fälle je 1000 Lebendgeburten in Island, Schweden, Finnland, Luxemburg und Japan bis zu Höchstwerten von 18 bzw. 23 Fällen in Mexiko und der Türkei (HE3.2). Auch in Polen, der Slowakischen Republik und den Vereinigten Staaten sind die Säuglingssterberaten relativ hoch. Alle OECDLänder haben in den letzten vier Jahrzehnten beachtliche Fortschritte bei der Senkung der Säuglingssterblichkeit erzielt, wobei in Portugal und Korea ein besonders starker Rückgang verzeichnet wurde.

In mehreren OECD-Ländern hat der Anteil der Neugeborenen mit niedrigem Geburtsgewicht im Verlauf der letzten Generation zugenommen (HE3.3). Für diesen Anstieg könnte es mehrere Gründe geben. Erstens ist die Zahl der Mehrlingsgeburten kontinuierlich gestiegen, was z.T. auf die Zunahme der Fruchtbarkeitsbehandlungen zurückzuführen ist. Zweitens nimmt das durchschnittliche Alter der Mütter bei der Geburt des ersten Kindes zu, wodurch sich wiederum das Risiko von Geburten mit niedrigem Gewicht erhöht. Drittens sind infolge neuer medizinischer Technologien und einer besseren pränatalen Versorgung die Überlebenschancen von Neugeborenen mit geringem Gewicht gestiegen.

Länder mit einem geringen Anteil an Neugeborenen mit niedrigem Geburtsgewicht weisen auch eine geringe Säuglingssterblichkeit auf (HE3.4). Japan bildet hier eine Ausnahme, dort ist der Anteil der Neugeborenen mit niedrigem Geburtsgewicht am höchsten, die Säuglingssterblichkeit aber dennoch gering. Der Anteil der Fälle von niedrigem Geburtsgewicht ist in Japan seit Ende der 1970er Jahre stark gestiegen - von 5\% der Neugeborenen auf fast 10\% im Jahr 2006. Dafür gibt es mehrere mögliche Gründe. Junge Frauen rauchen in Japan heute häufiger als früher, und das durchschnittliche Alter der Mütter bei der Geburt des ersten Kindes ist gestiegen (Jeong und Hurst, 2001).

\section{Weitere Informationen}

EURO-PERISTAT Project (2008), European Perinatal Health Report, www.europeristat.com/publications/europeanperinatal-health-report.shtml.

Jeong, H.S. und J. Hurst (2001), „An Assessment of the Performance of the Japanese Health Care System", OECD Labour Market and Social Policy Occasional Papers, No. 56, OECD, Paris.

\section{Anmerkungen zu den Abbildungen}

Abbildungen HE3.1 und HE3.2: In Kanada, Japan, den Vereinigten Staaten sowie einigen nordischen Ländern werden sehr kleine Frühgeborene mit geringen Überlebenschancen als Lebendgeburten gezählt, weshalb die angegebenen Werte dort höher sind als in Ländern, in denen dies nicht der Fall ist.

Abbildung HE3.1: 2005 für Australien, Kanada, Italien, Portugal und die Vereinigten Staaten; 2004 für Belgien, Frankreich und Schweden; 2003 für Luxemburg und die Türkei.

Abbildung HE3.2: 2005 für Belgien, Kanada und die Vereinigten Staaten; 2004 für Italien; 2002 für Korea. 


\section{GESUNDHEITSINDIKATOREN}

\section{Gesundheitszustand von Säuglingen}

HE3.1. Im OECD-Durchschnitt weist jedes 15. Neugeborene ein niedriges Geburtsgewicht auf Prozentsatz der Neugeborenen mit einem Geburtsgewicht von unter $2500 \mathrm{~g}, 2006$

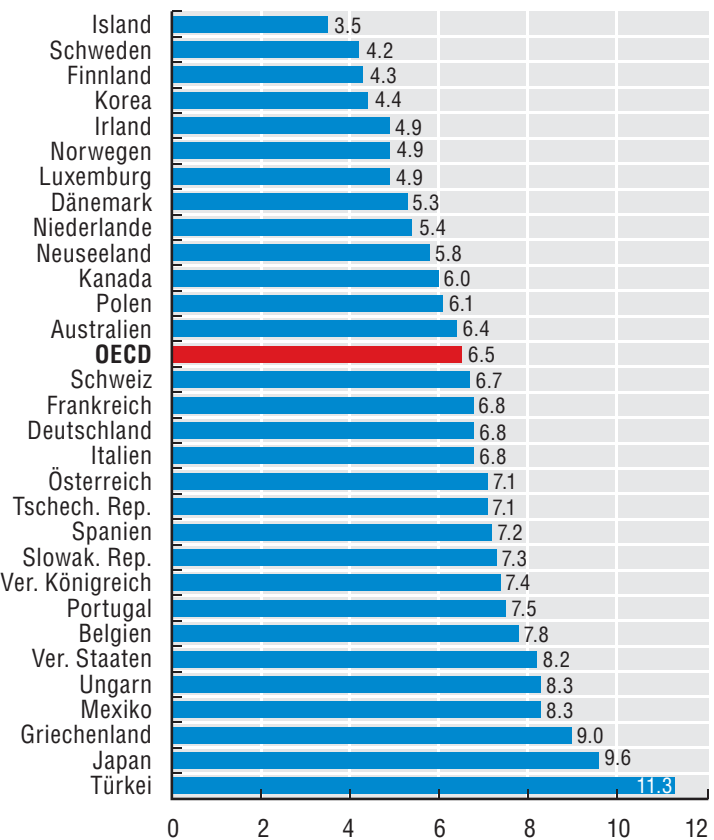

HE3.3. In mehreren OECD-Ländern ist die Zahl der Fälle von niedrigem Geburtsgewicht im Verlauf der letzten Generation gestiegen

Prozentsatz der Neugeborenen mit einem Geburtsgewicht von unter $2500 \mathrm{~g}, 2006$

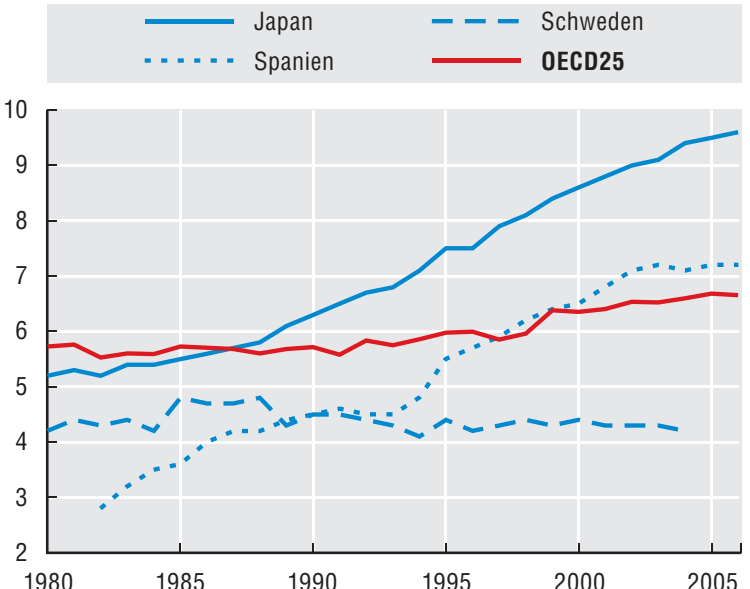

Quelle: OECD (2008), OECD-Gesundheitsdaten 2008, OECD, Paris (www.oecd.org/health/healthdata).
HE3.2. Im OECD-Durchschnitt stirbt eines von 200 Kindern im Säuglingsalter

Todesfälle je 1000 Lebendgeburten, 2006

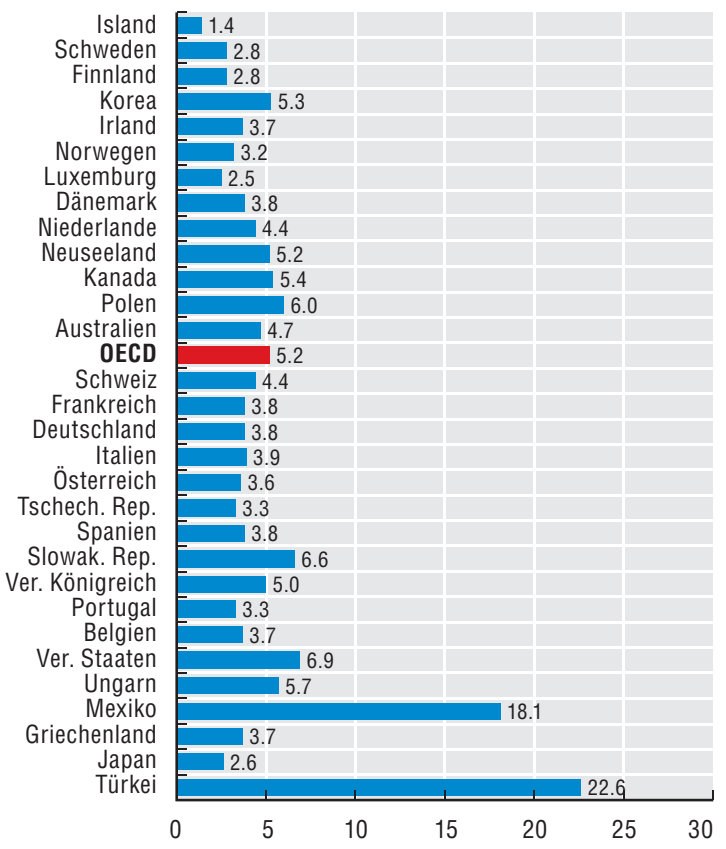

HE3.4. In Ländern mit wenigen Fällen von niedrigem Geburtsgewicht ist auch die Säuglingssterblichkeit gering

2006

Säuglingssterblichkeit (Todesfälle je 1000 Lebendgeburten)

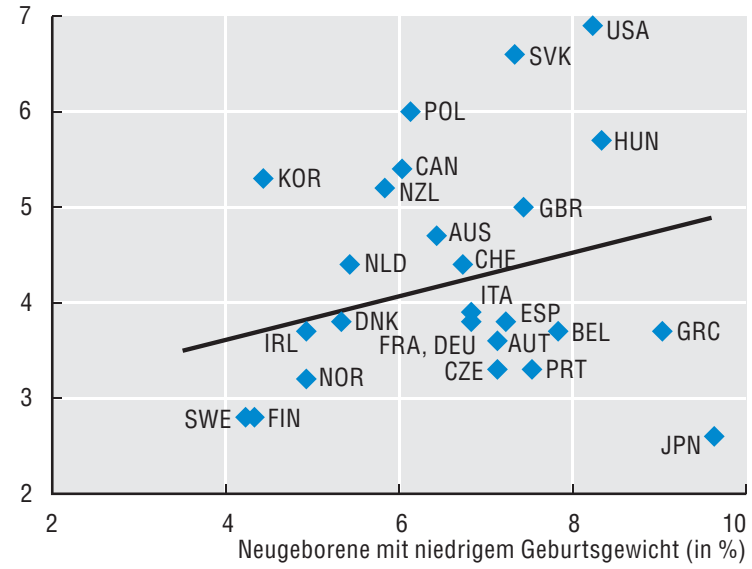

StatLink 에sद http://dx.doi.org/10.1787/550600314458 


\section{Definition und Messung}

Die am häufigsten verwendete Messgröße für Übergewicht und Fettleibigkeit stützt sich auf den Körpermassenindex (Body Mass Index - BMI). Der BMI ist definiert als Gewicht/Größe ${ }^{2}$ (wobei das Gewicht in Kilogramm und die Körpergröße in Metern ausgedrückt wird). Definitionsgemäß gelten Erwachsene mit einem BMI zwischen 25 und 30 als übergewichtig und Erwachsene mit einem BMI von über 30 als fettleibig (WHO, 1997). Diese Klassifizierung kann jedoch für einige ethnische Gruppen ungeeignet sein; die Schwellenwerte für Erwachsene sind zudem für Kinder nicht geeignet.

Für die meisten Länder beruhen die Schätzungen zu Übergewicht und Fettleibigkeit auf Selbstangaben zu Größe und Gewicht aus Gesundheitsbefragungen. Die Ausnahmen sind Australien, Luxemburg, Neuseeland, die Tschechische Republik, das Vereinigte Königreich und die Vereinigten Staaten, wo sich die Schätzungen auf die tatsächliche Messung der Größe und des Gewichts stützen. Die BMI-Schätzwerte, die auf der Messung von Größe und Gewicht basieren, sind im Allgemeinen höher und verlässlicher als Selbstangaben. Zum Beispiel lag die Fettleibigkeitsrate Erwachsener in den Vereinigten Staaten auf der Basis von direkten Befragungen 1999 bei 22\%, gegenüber $31 \%$ im selben Jahr auf der Grundlage der tatsächlichen Messungen.

In vielen OECD-Ländern hat sich die Zunahme der Fettleibigkeit zu einem großen öffentlichen Gesundheitsproblem entwickelt. Fettleibigkeit ist ein Risikofaktor für Bluthochdruck, hohen Cholesterinspiegel, Diabetes, HerzKreislauf-Krankheiten, Asthma, Arthritis sowie einige Krebsarten. In den Vereinigten Staaten ergab eine Studie, dass die Kosten der Fettleibigkeit die kombinierten Kosten des Rauchens und des übermäßigen Alkoholkonsums übersteigen (Sturm, 2002). Ende der 1990er Jahre machten die durch Fettleibigkeit verursachten Kosten für die Gesundheitsversorgung in den Vereinigten Staaten rd. 5-7\% der Gesamtgesundheitsausgaben und in Ländern wie Kanada, Australien und Neuseeland 2-3,5\% der Gesundheitsausgaben aus (Thompson und Wolf, 2001). Schätzungen aus den Vereinigten Staaten haben gezeigt, dass die Kosten der Gesundheitsdienste und der Medikamente für Fettleibige 36\% bzw. 77\% höher sind als für Normalgewichtige (Sturm, 2002).

In den meisten OECD-Ländern ist der Anteil der Übergewichtigen und Fettleibigen beträchtlich. Mindestens die Hälfte der Erwachsenenbevölkerung ist heute in folgenden Ländern übergewichtig oder fettleibig: Mexiko, Vereinigte Staaten, Vereinigtes Königreich, Australien, Griechenland, Neuseeland, Luxemburg, Ungarn, Tschechische Republik, Kanada, Deutschland, Portugal, Finnland, Spanien und Island. In den beiden asiatischen OECD-Ländern (Japan und Korea) sowie in einigen europäischen Ländern (Frankreich und Schweiz) ist der Anteil der Übergewichtigen und Fettleibigen niedriger. Bei alleiniger Betrachtung der Fettleibigkeit, bei der die gesundheitlichen Risiken größer sind als bei Übergewicht, zeigt sich, dass die Fettleibigkeitsprävalenzraten um den Faktor zehn variieren, von einem Tiefstwert von $4 \%$ in Korea und Japan bis zu über $30 \%$ in den Vereinigten Staaten und Mexiko (HE4.1).

Im Allgemeinen sind nicht mehr Frauen als Männer übergewichtig und fettleibig. In manchen Ländern übersteigt jedoch die Zahl der übergewichtigen und fettleibigen Männer die der Frauen (Griechenland), während in anderen Ländern das Gegenteil der Fall ist (Türkei, Mexiko) (HE4.2).

Immer mehr Personen sind übergewichtig und fettleibig. Die Fettleibigkeitsrate hat sich in den vergangenen zwanzig Jahren in den Vereinigten Staaten mehr als verdoppelt, in Australien fast verdreifacht und im Vereinigten Königreich mehr als verdreifacht (HE4.3). Auch in vielen westeuropäischen Ländern sind die Fettleibigkeitsraten in den vergangenen zehn Jahren erheblich gestiegen

In allen Bevölkerungsgruppen nimmt die Zahl der Übergewichtigen und Fettleibigen zu. Die Daten aus den Vereinigten Staaten, Kanada und dem Vereinigten Königreich deuten allerdings darauf hin, dass Übergewicht und Fettleibigkeit unter den Angehörigen benachteiligter sozioökonomischer Gruppen stärker verbreitet sind, insbesondere unter Frauen (Statistics Canada und Center for Disease Control and Prevention, 2004).

Auch immer mehr Kinder sind übergewichtig und fettleibig. Die Fettleibigkeit bei Kindern beläuft sich in den meisten OECD-Ländern auf zweistellige Raten, mit Spitzenwerten von einem Drittel bei Kindern im Alter von 1314 Jahren in Spanien (2000-2002), 29\% bei Kindern im Alter von 5-17 Jahren in England (2004) sowie etwa einem Viertel bei Kindern im Alter von 5-17 Jahren in Italien (1993-2001) und von 5-15 Jahren in Belgien (1998-1999) (International Association for the Study of Obesity, 2007).

\section{Weitere Informationen}

Australian Institute of Health and Welfare (2004), Australia's Health 2004, AIHW Cat. No. AUS 44, Canberra.

International Association for the Study of Obesity (2007), „International Obesity Taskforce Database“, verfügbar unter www.iotf.org/documents/Europeandatatable_000.pdf (Internetzugriff 11. Juni 2007).

Statistics Canada und Center for Disease Control and Prevention (2004), Joint Canada/United States Survey of Health, 2002-2003, Statistics Canada Cat. 82M0022-XIE, Ottawa.

Sturm, R. (2002), „The Effects of Obesity, Smoking and Drinking on Medical Problems and Costs", Health Affairs, Vol. 21, No. 2, S. 245-253.

Thompson, D. und A.M. Wolf (2001), „The Medicalcare Burden of Obesity“, Obesity Reviews, Vol. 2, S. 189-197.

Weltgesundheitsorganisation (1997), Obesity: Preventing and Managing the Global Epidemic, WHO, Genf.

\section{Anmerkungen zu den Abbildungen}

Abbildungen HE4.1 und HE4.2: Anmerkung 1: Für Australien, Luxemburg, Neuseeland, die Tschechische Republik, das Vereinigte Königreich und die Vereinigten Staaten basieren die Abbildungen auf der tatsächlichen Messung der Größe und des Gewichts und nicht auf Selbstangaben. 


\section{GESUNDHEITSINDIKATOREN}

\section{HE4.1. Hohe Fettleibigkeitsraten in vielen OECD-Ländern}

Prozentsatz der Erwachsenenbevölkerung mit einem BMI>30

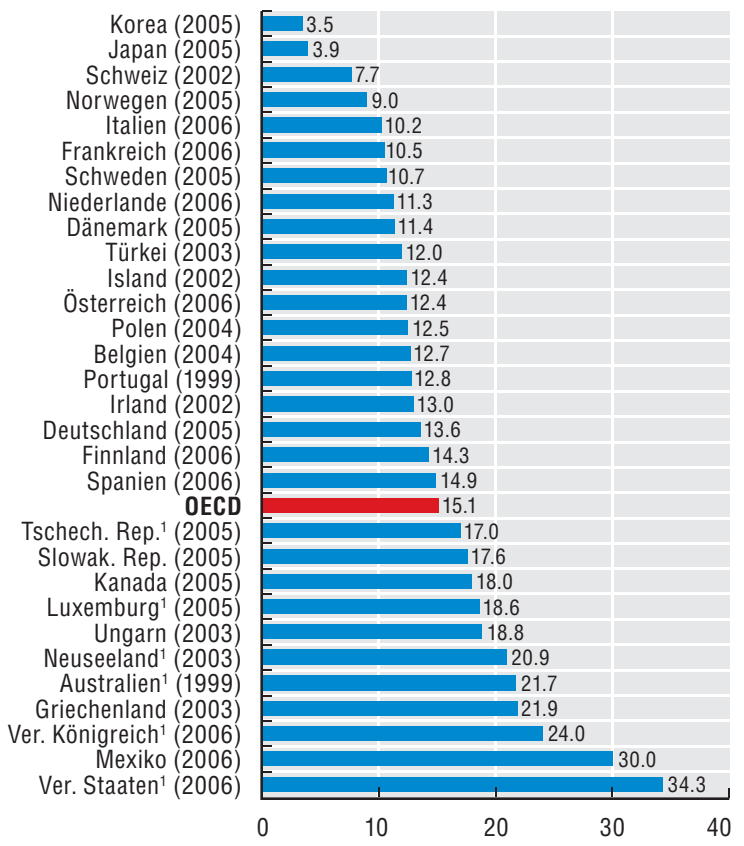

\section{HE4.2. Im Allgemeinen sind nicht mehr Frauen als Männer fettleibig}

Differenz zwischen den Prozentsätzen von Frauen und Männern mit einem $B M I>30$

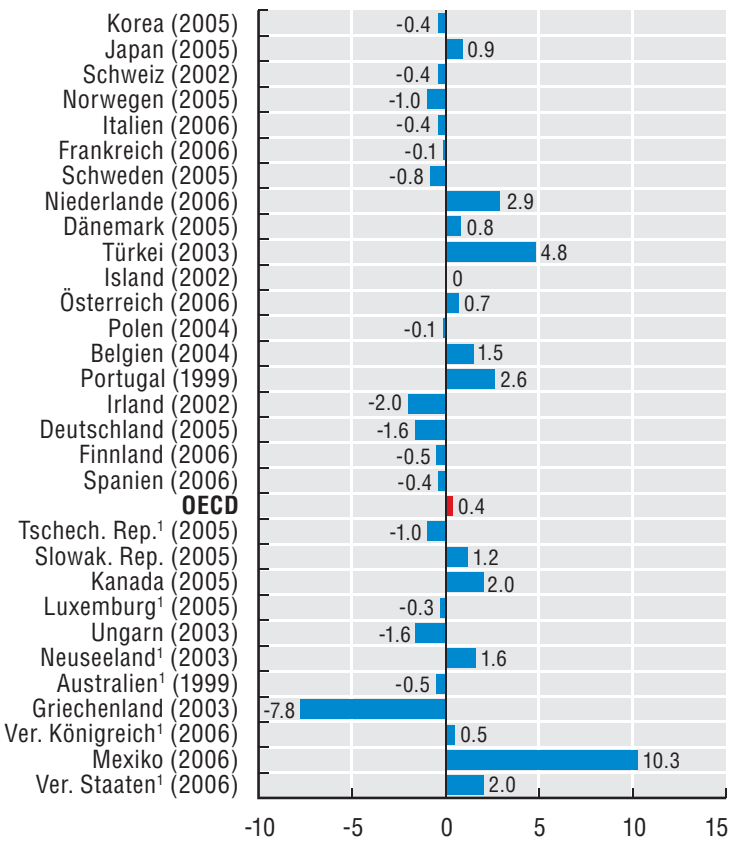

HE4.3. Steigende Fettleibigkeitsraten in den OECD-Ländern

Prozentsatz der Erwachsenenbevölkerung mit einem BMI von über 30, im Zeitverlauf, verschiedene Jahre

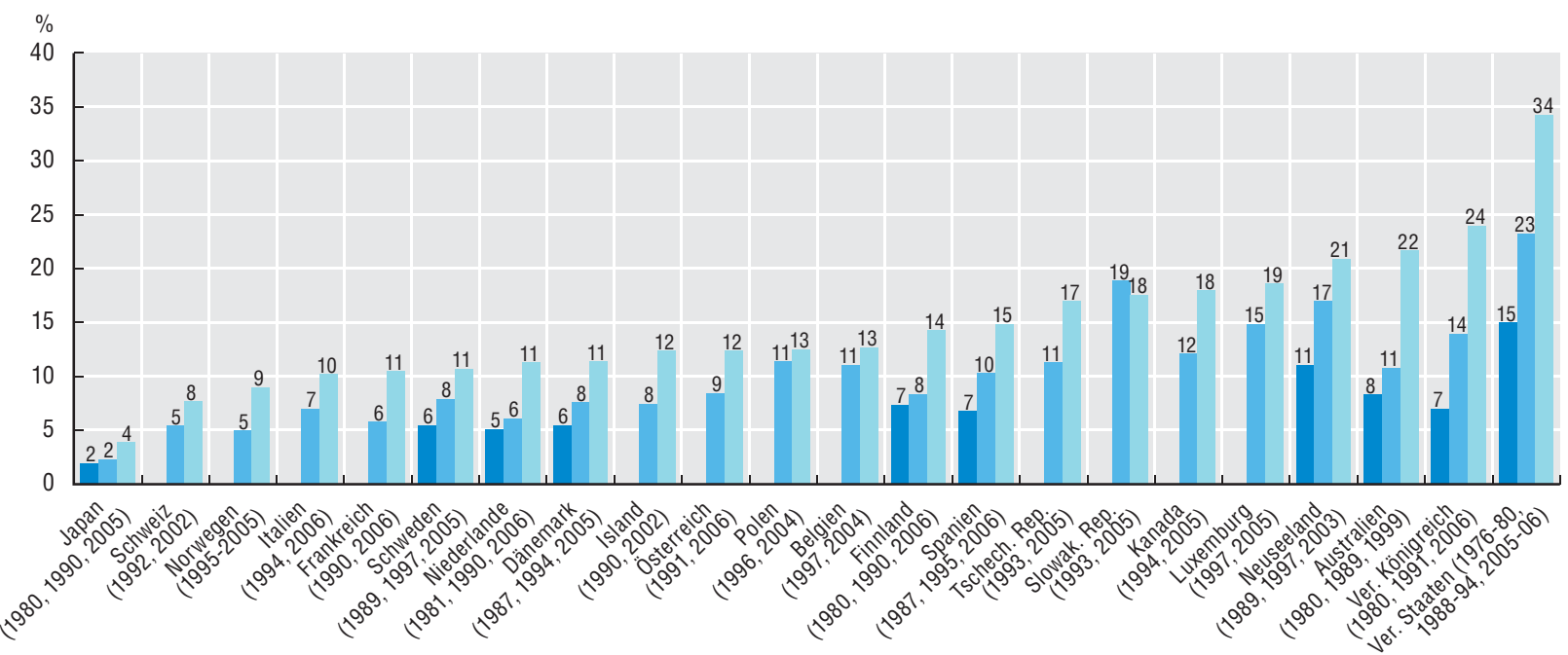

Quelle: OECD (2008), OECD-Gesundheitsdaten 2008, CD-Rom,

OECD, Paris (www.oecd.org/health/healthdata). 


\section{Definition und Messung}

Die Daten zur Körpergröße beziehen sich auf Personen im Alter von 20-49 Jahren: Das Längenwachstum kann noch bis zum 20. Lebensjahr erfolgen, und ab dem 50. Lebensjahr beginnt der Körper wieder zu schrumpfen. Die gemessene Körpergröße genießt gegenüber der selbst angegebenen Körpergröße insofern den Vorzug, als die Daten darauf hindeuten, dass die Befragten ihre eigene Statur in der Regel zu hoch einschätzen (Gorber et al., 2007). Diese Verzerrungen infolge von Selbstangaben variieren je nach Alter, Geschlecht, Bildungsniveau, Befragungsmethode und Zweck der Umfrage. Die Daten aus einer neueren systematischen Studie legen den Schluss nahe, dass die ungewichtete durchschnittliche Überzeichnung der Körpergröße infolge von Selbstangaben von Männern und Frauen in beiden Fällen etwa $1 \mathrm{~cm}$ beträgt (Gorber et al, 2007). Wenn die Körpergröße von Kohorten im Alter von 2024 Jahren mit der von Kohorten im Alter von 4549 Jahren verglichen wird, um die Größenentwicklung der Erwachsenen in jüngster Zeit zu untersuchen, können manche oder sämtliche dieser Messfehler durch die Differenzenmethode beseitigt werden. Soweit möglich wurden die Daten direkt spezifischen amtlichen Gesundheitserhebungen entnommen.

Die Körpergröße von Erwachsenen wird sowohl durch das genetische Potenzial als auch durch die Nettoernährung während der Kindheit bestimmt. Die Nettoernährung entspricht der Qualität und Quantität der Bruttoernährung abzüglich der Verluste durch körperliche Aktivität und Krankheit. Folglich ist die Umweltkomponente der Körpergröße von Erwachsenen eine kumulierte Zusammenfassung des Ernährungs- und Krankheitsumfelds eines Kindes. (Steckel, 1995; Silventoinen, 2003). Wenn das Ernährungs- und Krankheitsumfeld Kinder in Form von abnehmenden Erträgen beeinflusst, dienen die Ländervergleiche der durchschnittlichen Körpergröße von Erwachsenen auch dazu, die umweltbezogenen Ungleichheiten für Kinder innerhalb eines Landes anzuzeigen.

Die Körpergröße von Erwachsenen unterscheidet sich erheblich zwischen den einzelnen OECD-Ländern. Männer in den nordischen und nordeuropäischen Ländern sind mit einer Körpergröße von über 1,80 Meter am größten (HE5.1). Die kleinsten Männer sind in Mexiko, Portugal, Korea und Japan zu finden, wo die Körpergröße $10 \mathrm{~cm}$ oder mehr unter der des Landes mit der größten Körpergröße liegt. Abbildung HE5.1 zeigt darüber hinaus ähnliche länderspezifische Muster für Frauen, wobei die größten Frauen 1,68 Meter und die kleinsten Frauen 1,54 Meter groß sind. Der Größenvorteil der nordischen und nordeuropäischen Länder könnte z.T. auf die effektive Gesundheitsversorgung und die wirksamen sozialen Sicherungssysteme zurückzuführen sein. Unter den Ländern mit einer kleineren Körpergröße ist in Mexiko ein vergleichsweise niedriges Pro-KopfEinkommen festzustellen, während Japan und Korea im Vergleich zu den restlichen OECD-Ländern günstiger dastehen. Es gibt wenig stichhaltige Belege dafür, dass die Unter- schiede bei der Körpergröße zwischen den einzelnen Ländern durch die durchschnittlichen länderspezifischen Unterschiede bei der genetischen Ausstattung bedingt sind (Deaton, 2007). Insgesamt sind Männer immer größer als Frauen. Das Verhältnis der Körpergröße von Männern gegenüber der von Frauen reicht von 1,06 in Portugal bis zu 1,09 in Australien, Mexiko und den Niederlanden. Dieser Unterschied zwischen den Geschlechtern weist eine starke genetische Komponente auf.

Die Einwohner der OECD-Länder werden größer. Beim Vergleich der Kohorte im Alter von 45-49 Jahren mit der Kohorte im Alter von 20-24 Jahren zeigt sich, dass die durchschnittliche Körpergröße im OECD-Raum in einem Zeitraum von 25 Jahren für Männer um $3 \mathrm{~cm}$ und für Frauen um $2 \mathrm{~cm}$ zugenommen hat. Dieser Zuwachs deutet in den einzelnen Ländern auf eine Verbesserung der Nettoernährung während der Kindheit hin. Spitzenreiter ist Korea, wo die jungen Männer $6 \mathrm{~cm}$ größer als die Generation ihrer Väter und die jungen Frauen $4 \mathrm{~cm}$ größer als die Generation ihrer Mütter sind. Das Schlusslicht bilden die Vereinigten Staaten, wo von einer Generation zur nächsten kein Zuwachs der Körpergröße verzeichnet wurde (Komlos, 2008). Die Stagnation im Längenwachstum in den Vereinigten Staaten lässt sich nicht mit der Zuwanderung von verhältnismäßig kleinen Personen in jüngster Zeit erklären

Die „kleinen“ Länder holen die „großen“ Länder ein. Aus dem Streudiagramm in Abbildung HE5.2 ist zu ersehen, dass die Körpergröße der ursprünglich kleineren Einwohner Koreas, Mexikos, Spaniens und Portugals rascher zunimmt als die der Einwohner Schwedens, Islands und Dänemarks. Die Körpergröße der Männer konvergiert schneller als die der Frauen. Sowohl für Männer als auch für Frauen zeigt die Regressionsgerade, die die Konvergenz wiedergibt, die relativ geringen Zuwachsraten in Mexiko, Japan und den Vereinigten Staaten. In Anbetracht der ursprünglichen Körpergröße ihrer Bevölkerung erzielten Korea, Spanien und Irland für Männer sowie Korea, Spanien und Belgien für Frauen die höchsten Zuwachsraten.

\section{Weitere Informationen}

Deaton, A. (2007), „Height, Health, and Development“, Proceedings of the National Academy of Sciences, Vol. 104, S. 13232-13237.

Gorber, S.C. et al. (2007), „A Comparison of Direct vs. Selfreport Measures for Assessing Height, Weight, and BMI: A Systematic Review“, Obesity Reviews, Vol. 8, No. 4, S. 307-326.

Komlos, J. (2008), „Stagnation in Heights amongst SecondGeneration US-born Army Personnel“, Social Science Quarterly, Vol. 89, No. 2, S. 445-455.

Silventoinen, K. (2003), „Determinants of Variation in Adult Height“, Journal of Biosocial Science, Vol. 35, S. 263-285.

Steckel, R. (1995), "Stature and the Standard of Living“, Journal of Economic Literature, Vol. 33, No. 4, S. 1903-1940.

\section{Anmerkungen zu den Abbildungen}

Abbildung HE5.1: M = gemessene Körpergröße, S = selbst angegebene Körpergröße und $U$ = unbekannte Methode der Körpergrößenmessung. 
HE5.1. Die Einwohner der nordischen und der europäischen Länder sind am größten Mittlere Körpergröße von Männern im Alter von 20-49 Jahren

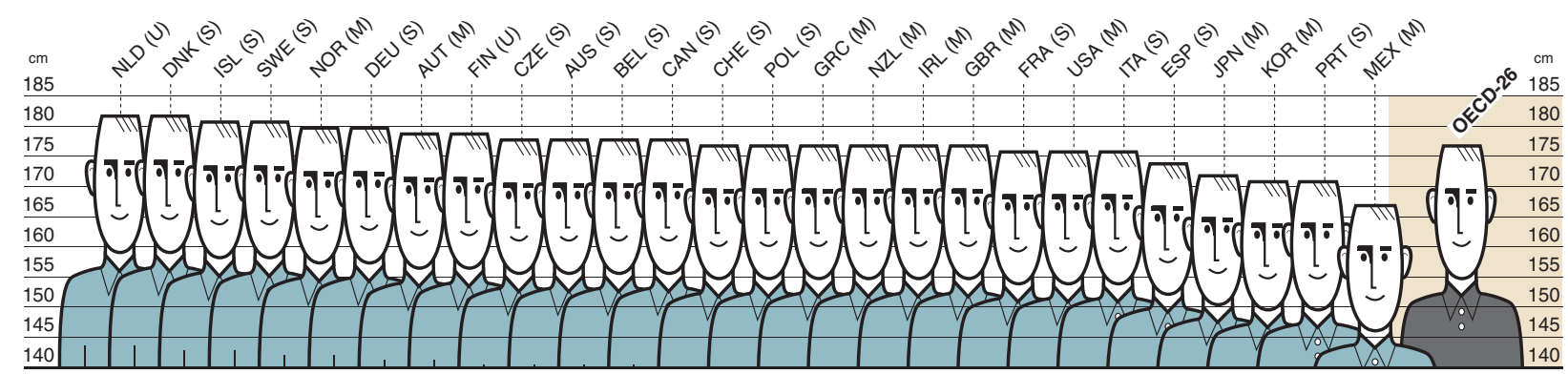

Mittlere Körpergröße von Frauen im Alter von 20-49 Jahren

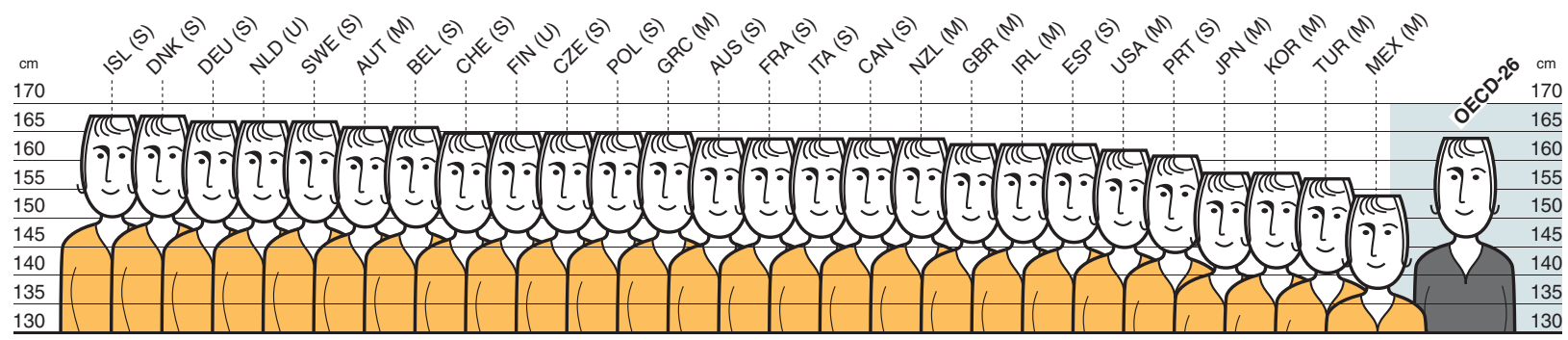

HE5.2. Körpergröße von Männern und Frauen konvergiert gegen die der Länder mit der größten Körpergröße

Zuwachsrate zwischen den Kohorten im Alter von 45-49 Jahren und 20-24 Jahren, in \%

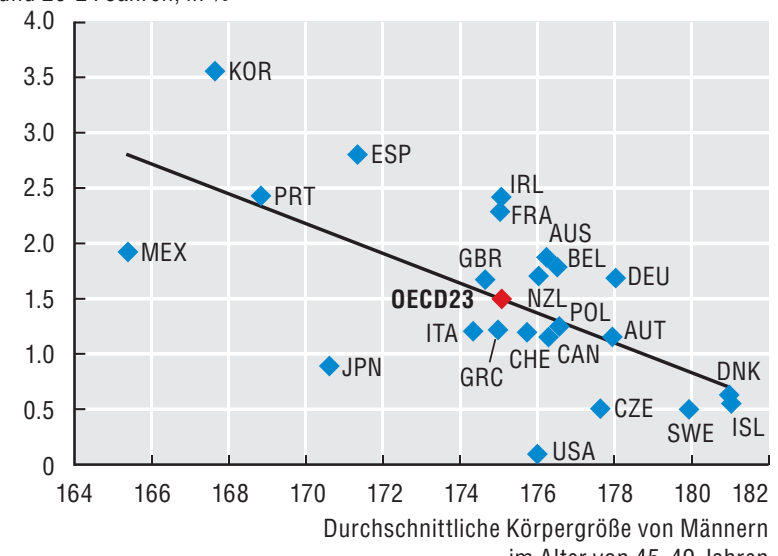

im Alter von 45-49 Jahren
Zuwachsrate zwischen den Kohorten im Alter von 45-49 Jahren und 20-24 Jahren, in \%

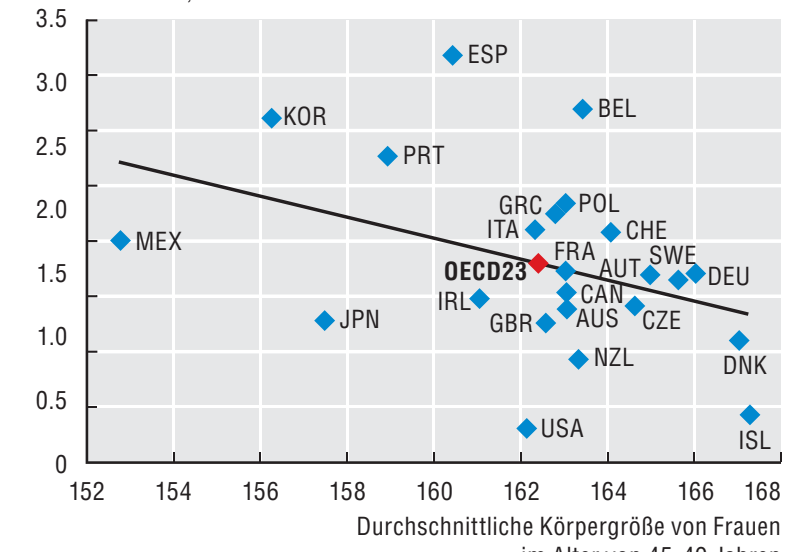

im Alter von 45-49 Jahren 


\section{Definition und Messung}

Die erste verwendete Datenreihe stammt aus groß angelegten epidemiologischen Erhebungen im Rahmen der World Mental Health Survey Initiative (WMHSI) der Weltgesundheitsorganisation. Diese Erhebungen wurden im Zeitraum 2002-2005 in zehn OECD-Ländern durchgeführt (drei weitere OECDLänder nehmen in diesem Bereich eigene Erhebungen vor). Dabei wurde ein gemeinsames Diagnoseinstrument, das WHO Composite International Diagnostic Instrument (CIDI), verwendet, um Datenmaterial über das Vorkommen verschiedener Arten psychischer Störungen, ihre Merkmale und Intensität sowie die erfolgte Behandlung zu erhalten.

$\mathrm{Zu}$ den untersuchten Störungen gehören Angststörungen, Störungen der Stimmungsregulation, Störungen der Impulskontrolle und Störungen, die auf den Konsum von Alkohol und Drogen zurückzuführen sind. Alle Störungen sind in Schweregrade eingestuft (schwere, mäßige oder leichte Störungen).

Die WMHSI-Daten beziehen sich in der Regel auf alle Personen ab 18 Jahre. In Neuseeland liegt die Altersgrenze jedoch bei 16 Jahren, in Japan bei 20 Jahren und in Mexiko bei 18-65 Jahren. Die Stichprobengrößen umfassen zwischen rd. 2000 Personen (Niederlande) und 13000 Personen (Neuseeland). Die Antwortquoten variieren zwischen $50 \%$ (Belgien) und $80 \%$ (Neuseeland). Die Stichproben sind in den meisten Ländern national repräsentativ, in Mexiko dagegen repräsentativ für alle städtischen Gebiete und in Japan für vier städtische Ballungsräume. Die Tatsache, dass bei den europaweiten Erhebungen bipolare Störungen und Substanzmissbrauch unberücksichtigt bleiben, schränkt die Vergleichbarkeit erheblich ein.

Die zweite verwendete Reihe von Daten, die Ende 2007 gesammelt wurden, stammt aus der europaweiten Erhebung zur Lebensqualität der Europäischen Stiftung zur Verbesserung der Lebens- und Arbeitsbedingungen. Sie erstreckt sich zwar auf eine viel größere Anzahl von Mitgliedsländern, beschränkt sich aber auf die europäischen Länder und die Türkei. Der aus dieser Erhebung resultierende Index stützt sich auf Selbstangaben zu fünf Fragen, die auf einem kurzen WHO-Index basieren, der sich auf Symptome depressiver Art bezieht.

Störungen der seelischen Gesundheit stellen für die Betroffenen und die Gesellschaft eine große Belastung dar. Die wirtschaftlichen Kosten psychischer Gesundheitsprobleme - einschließlich Behandlungskosten und indirekter Kosten auf Grund von Produktivitätsminderung und
Arbeitsausfalltagen - werden im Vereinigten Königreich auf über 2\% des BIP und in Kanada auf etwas weniger geschätzt (WHO, 2007).

Psychische Gesundheitsprobleme sind keine Randerscheinung. Der Anteil der Personen, die angaben, im vergangenen Jahr in der einen oder anderen Art psychische Gesundheitsprobleme gehabt zu haben, reichte von $8 \%$ in Italien bis zu $26 \%$ in den Vereinigten Staaten (HE6.1). Noch höher war die Anzahl der Personen, die eigenen Angaben zufolge während ihres bisherigen Lebens psychische Gesundheitsprobleme verschiedenster Art hatten, wobei dieser Anteil von 18\% in Italien und Japan bis rd. $40 \%$ oder mehr in Neuseeland und den Vereinigten Staaten reichte.

In allen Ländern sind die am häufigsten auftretenden Störungen durch Angstzustände bedingt, gefolgt von Störungen der Stimmungsregulation (HE6.2). Seltener sind Störungen, die auf Impulskontrolldefizite und Substanzkonsum zurückzuführen sind. Ein hoher Anteil aller psychischen Störungen wurde als geringfügig eingestuft, doch 4\% der Bevölkerung der an der Untersuchung teilnehmenden Länder haben eigenen Angaben zufolge mäßige Störungen und weitere $3 \%$ schwere Störungen - mit einer Prävalenz von schweren Störungen in den Vereinigten Staaten, die mehr als doppelt so hoch ist.

Der überwiegende Teil der seelischen Störungen bleibt unbehandelt (HE6.3). Der Behandlungsanteil ist zwar bei schweren und mäßigen Störungen im Durchschnitt höher (48\% bzw. 31\%), doch bleiben viele schwere Fälle unbehandelt.

Der Index der seelischen Gesundheit für 21 Länder ergibt für die Türkei einen relativ schlechten psychischen Gesundheitszustand und für Norwegen einen guten psychischen Gesundheitszustand (HE6.3). Ein schlechter psychischer Gesundheitszustand ist in geringerem Maße für Italien und Polen festzustellen, und ein guter psychischer Gesundheitszustand ist in geringerem Umfang in den Niederlanden, Irland und Deutschland zu beobachten. Im Mittelfeld des Index der seelischen Gesundheit sind die Unterschiede zwischen den Ländern gering.

\section{Weitere Informationen}

Kessler, R.C. et al. (2007), „Lifetime Prevalence and Age-ofOnset Distributions of Mental Disorders in the World Health Organisation World Mental Health Survey Initiative“, World Psychiatry, Vol. 6, Oktober.

OECD (2008), „Are All Jobs Good for Your health? The Impact of Work Status and Working Conditions on Mental Health“, OECD Employment Outlook, OECD, Paris.

WHO World Mental Health Survey Consortium (2007), „Prevalence, Severity, and Unmet Need for Treatment of Mental Disorders in the World Health Organisation World Mental Health Surveys“, American Medical Association, Vol. 291, No. 21, Juni. 
HE6.1. Jahres- und Lebenszeitprävalenz psychischer Gesundheitsprobleme in zehn OECD-Ländern

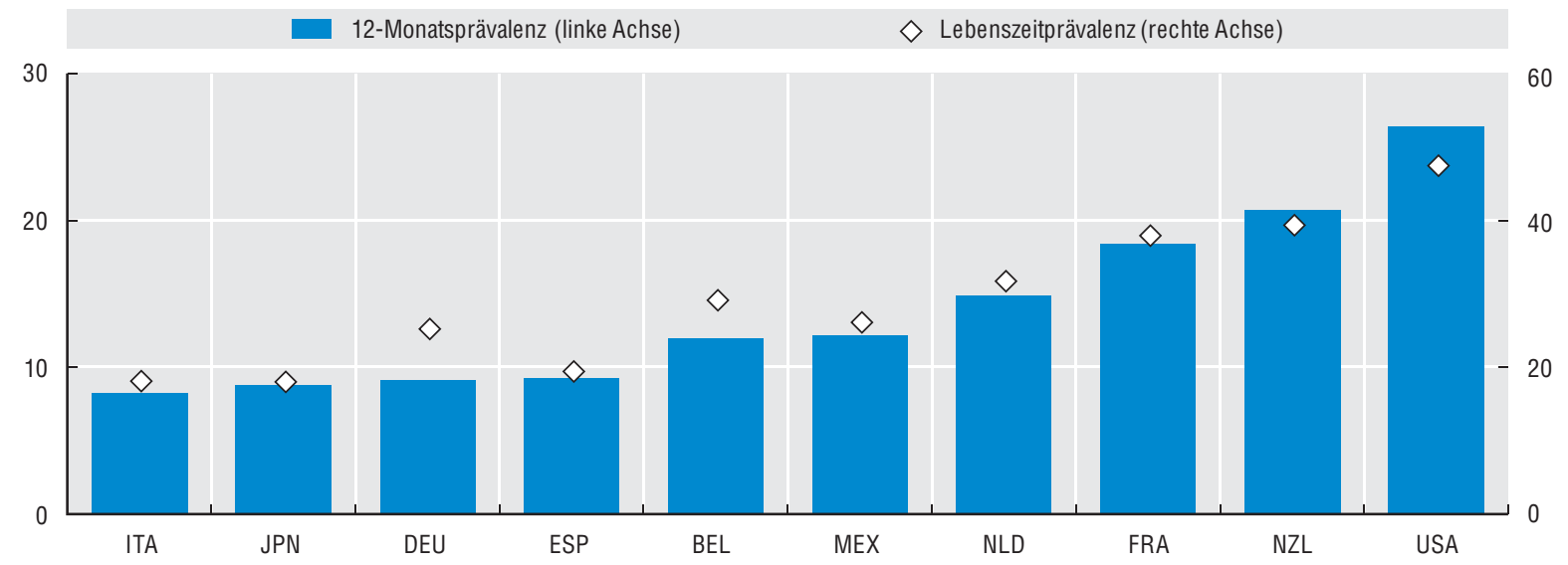

Quelle: WHO World Mental Health Survey Consortium (2007).

HE6.2. Art, Schweregrad und Behandlung psychischer Gesundheitsprobleme in zehn OECD-Ländern

\begin{tabular}{|c|c|c|c|c|c|c|c|c|c|c|c|c|c|}
\hline & \multicolumn{8}{|c|}{ 12-Monatsprävalenz, prozentualer Anteil der Gesamtbevölkerung } & \multirow{2}{*}{\multicolumn{5}{|c|}{$\begin{array}{c}\text { Anteil der Personen mit psychischen } \\
\text { Gesundheitsstörungen in Behandlung } \\
\text { Nach Schweregrad }\end{array}$}} \\
\hline & \multicolumn{5}{|c|}{ Nach Art } & \multicolumn{3}{|c|}{ Nach Schweregrad } & & & & & \\
\hline & $\begin{array}{c}\text { Angst- } \\
\text { zustände }\end{array}$ & $\begin{array}{l}\text { Stimmungs- } \\
\text { regulation }\end{array}$ & $\begin{array}{l}\text { Impuls- } \\
\text { kontrolle }\end{array}$ & $\begin{array}{l}\text { Substanz- } \\
\text { missbrauch }\end{array}$ & $\begin{array}{c}\text { Andere } \\
\text { Störungen }\end{array}$ & Schwer & Mäßig & Leicht & Schwer & Mäßig & Leicht & $\begin{array}{c}\text { Keine } \\
\text { Störungen }\end{array}$ & Insgesamt \\
\hline Belgien & 7 & 6 & 1 & 1 & 12 & 2 & 3 & 6 & 54 & 50 & 28 & 7 & 11 \\
\hline Deutschland & 6 & 4 & 0 & 1 & 9 & 1 & 3 & 5 & 50 & 31 & 28 & 5 & 8 \\
\hline Frankreich & 12 & 9 & 1 & 1 & 18 & 3 & 6 & 10 & 63 & 36 & 22 & 8 & 12 \\
\hline Italien & 6 & 4 & 0 & 0 & 8 & 1 & 3 & 4 & .. & 31 & 19 & 2 & 5 \\
\hline Japan & 5 & 3 & 1 & 2 & 9 & 2 & 4 & 3 & .. & 17 & 11 & 5 & 6 \\
\hline Mexiko & 7 & 5 & 1 & 3 & 12 & 4 & 4 & 5 & 20 & 19 & 10 & 3 & 4 \\
\hline Neuseeland & 15 & 8 & 1 & 4 & 23 & 0 & 0 & 0 & .. & .. & .. & .. & .. \\
\hline Niederlande & 9 & 7 & 1 & 3 & 15 & 2 & 4 & 9 & 50 & 35 & 27 & 7 & 11 \\
\hline Spanien & 6 & 5 & 1 & 0 & 9 & 1 & 3 & 5 & 65 & 38 & 35 & 4 & 7 \\
\hline Ver. Staaten & 18 & 10 & 7 & 4 & 26 & 8 & 9 & 9 & 52 & 34 & 23 & 8 & 15 \\
\hline Durchschnitt & 9 & 6 & 2 & 2 & 15 & 3 & 4 & 6 & 48 & 31 & 21 & 6 & 9 \\
\hline
\end{tabular}

Quelle: WHO World Mental Health Survey Consortium (2007).

\section{HE6.3. Index der seelischen Gesundheit für 21 europäische OECD-Länder}

In Prozent, 2007, ein höherer Wert entspricht einer besseren seelischen Gesundheit

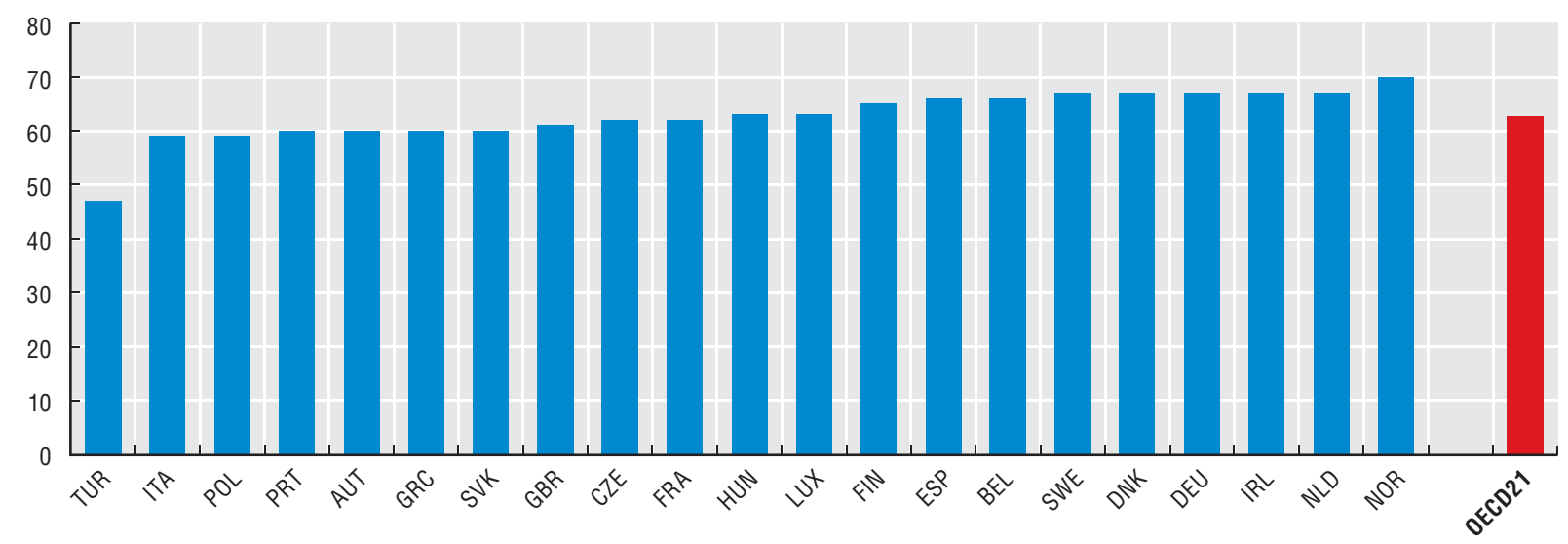

Quelle: Zweite europaweite Erhebung zur Lebensqualität, Europäische Stiftung zur Verbesserung der Lebens- und Arbeitsbedingungen, 2007, www.eurofound.europa.eu/publications/htmlfiles/ef0852.htm. 


\section{Definition und Messung}

Langzeitpflegebedürftige sind Personen, die über einen längeren Zeitraum auf Grund von Einschränkungen ihrer funktionellen körperlichen oder geistigen Kapazität formelle entgeltliche Pflegeleistungen erhalten. Sie benötigen Hilfe bei Verrichtungen wie Waschen, Anziehen, Essen, Hilfe beim Hinlegen/Hinsetzen in und Aufstehen aus Bett und Sessel, bei der Fortbewegung sowie bei der Toilettenbenutzung. Diese Pflegeleistungen werden häufig in Kombination mit medizinischen Grundleistungen erbracht. Die Langzeitpflege kann in einer Einrichtung oder zu Hause erfolgen.

Die internationale Vergleichbarkeit der Daten ist begrenzt. Die Daten beziehen sich im Allgemeinen auf einen bestimmten Tag im Jahr, für Dänemark jedoch auf eine Woche, für Japan auf einen Monat und für Ungarn und Neuseeland sowie für Bezieher häuslicher Pflegeleistungen in der Tschechischen Republik und der Schweiz auf das ganze Jahr. Für die Tschechische Republik, Italien und die Slowakische Republik sind Daten über Langzeitpflegebedürftige jeden Alters verfügbar. In Österreich, Belgien und Polen liegt die Altersgrenze für ältere Menschen bei 60 Jahren (anstatt 65 Jahren), während sie in Norwegen 67 Jahre (anstatt 65 Jahre) und in Polen 75 Jahre (anstatt 80 Jahre) beträgt. Darüber hinaus umfassen die Daten in manchen Ländern (Deutschland, Finnland, Irland, Polen, Schweiz, Tschechische Republik, Vereinigtes Königreich und Vereinigte Staaten) Empfänger von Pflegeleistungen, die aus privaten Mitteln finanziert werden.

Bei den Langzeitpflegesystemen bestehen enorme Unterschiede zwischen den einzelnen OECD-Ländern. Die nordischen Länder verfügen über umfassende Langzeitpflegesysteme. 2006 betrug der Anteil der Pflegeempfänger in diesen Ländern mehr als 15\% der Personen im Alter von über 65 Jahren (Abbildung HE7.1). Andere Länder mit allgemeinen und umfassenden Langzeitpflegesystemen (z.B. Deutschland, Japan, Luxemburg, die Niederlande und Österreich) weisen ebenfalls einen relativ hohen Anteil an Pflegeempfängern auf. Im Gegensatz dazu liegt der Anteil der Personen im Alter von über 65 Jahren, die Langzeitpflegeleistungen erhalten, in Korea, Italien und den osteuropäischen Ländern (außer Ungarn), wo formelle Vorkehrungen für die Langzeitpflege nicht verbreitet sind, zwischen $0,6 \%$ und 3,6\%.

Die Anteile der alten Menschen in Langzeitpflege konvergieren. Der Gesamtanteil der Langzeitpflegebedürftigen stieg in Ländern, in denen um das Jahr 2000 ein relativ geringer Anteil festzustellen war (Australien, Belgien, Island, Italien, Japan und Korea). Dagegen ging er in Ländern zurück, in denen der Anteil im Jahr 2000 über dem OECD-Durchschnitt lag (Deutschland, Finnland, Norwegen, Schweden und Schweiz) ebenso wie in Irland und den Vereinigten Staaten.

Häusliche Pflege überwiegt in den meisten OECD-Ländern. In Australien, Belgien, Italien, Japan, Korea und Schweden kann eine Verschiebung von institutioneller zu häuslicher Pflege beobachtet werden. Diese Verschiebung spiegelt sowohl die Präferenzen der älteren Menschen als auch den Versuch wider, den Rückgriff auf eine kostspielige institutionelle Pflege zu reduzieren (OECD, 2005). In
Deutschland allerdings war in jüngster Zeit ein gegenläufiger Trend zu beobachten (Gibson und Redfoot, 2007).

Die „älteren“ alten Menschen befinden sich mit größerer Wahrscheinlichkeit in Langzeitpflege. In der ältesten Alterskohorte (ab 80 Jahre) ist der durchschnittliche Anteil der Langzeitpflegebedürftigen im OECD-Raum über fünfmal höher als der Anteil der Pflegebedürftigen im Alter zwischen 6579 Jahren. Der Anteil der Pflegebedürftigen in der Alterskohorte ab 80 Jahre ist in den meisten Ländern gesunken, außer in Australien, Island und Japan (Abbildung HE7.2). Ein ähnlicher rückläufiger Anteil der Pflegebedürftigen ist in der Kohorte im Alter von 65-79 Jahren festzustellen.

Mehr Frauen als Männer sind in Langzeitpflege. Der durchschnittliche Anteil der Bezieher von Pflegeleistungen unter Frauen im Alter zwischen 65-79 Jahren ist um über ein Drittel höher als der unter Männern. Diese Kluft wird unter Pflegebedürftigen ab 80 Jahre größer, wo der durchschnittliche Anteil der Frauen anderthalbmal so hoch ist wie der der Männer (Abbildung HE7.3). Diese Überrepräsentation steht mit einer höheren Prävalenz von Behinderungen unter älteren Frauen im Einklang (Lafortune et al., 2007).

\section{Weitere Informationen}

Gibson, M.J. und Redfoot, D.L. (2007), Comparing Long-Term Care in Germany and the United States: What Can We Learn From Each Other?, AARP, Washington, DC.

Lafortune, G. et al. (2007), „Trends in Severe Disability Among Elderly People: Assessing the Evidence in 12 OECD Countries and the Future Implications", OECD Health Working Papers No. 26, OECD, Paris.

OECD (2005), Long-term Care for Older People, OECD, Paris.

\section{Anmerkungen zu den Abbildungen}

Abbildung HE7.1 bis HE7.3: Die Daten über Bezieher häuslicher Pflegeleistungen sind für Irland, Island, Kanada, die Slowakische Republik und die Vereinigten Staaten nicht verfügbar, und für HE7.1 sind die Daten über Bezieher häuslicher Pflegeleistungen für die Schweiz nicht verfügbar. Die Daten über Personen, die Pflegeleistungen in Einrichtungen in Anspruch nehmen, beziehen sich auf 1999 (Vereinigte Staaten) und 2001 (Irland) anstatt auf 2000 sowie auf 2003 (Kanada) und 2004 (Vereinigte Staaten) anstatt auf 2006. Die Daten über Empfänger institutioneller und häuslicher Pflegeleistungen beziehen sich auf 2001 (Vereinigtes Königreich) und 2002 (Australien und Japan) anstatt auf 2000 sowie auf 2003 (Frankreich, Österreich und Slowakische Republik), 2004 (Belgien, Korea und Vereinigtes Königreich) und 2005 (Australien und Schweiz) anstatt auf 2006. Wegen weiterer Datenspezifikationen vgl. die über den StatLink verfügbaren Informationen. Anmerkung 1: Die Daten beziehen sich auf unterschiedliche Altersaufschlüsselungen. Für Frankreich beziehen sich die Daten über Empfänger häuslicher Pflegeleistungen auf Pflegebedürftige ab 60 Jahre, während die Daten über Pflegebedürftige in Einrichtungen Personen ab 65 Jahre betreffen. Entsprechende Bevölkerungsdaten werden verwendet, um den Anteil zu berechnen, außer für Norwegen, wo auf Personen ab 65 Jahre (anstatt 67 Jahre) zurückgegriffen wird, um den Anteil zu ermitteln, was eine Unterschätzung zur Folge hat. Anmerkung 2: Die Daten beziehen sich nicht auf einen bestimmten Tag im Jahr, was eine Überzeichnung zur Folge hat. Anmerkung 3: Die Daten umfassen Pflegebedürftige, die ihre Pflegeleistungen vollständig mit privaten Mitteln bezahlen. Für die Tschechische Republik umfassen nur die Daten über häusliche Pflege Empfänger von Pflegeleistungen, die mit privaten Mitteln finanziert werden. 
HE7.1. Die meisten Empfänger formeller Langzeitpflegeleistungen werden zu Hause gepflegt

Personen ab 65 Jahre, die in Einrichtungen leben bzw. zu Hause formelle Pflege erhalten, als Anteil der Personen ab 65 Jahre, 2000 und 2006

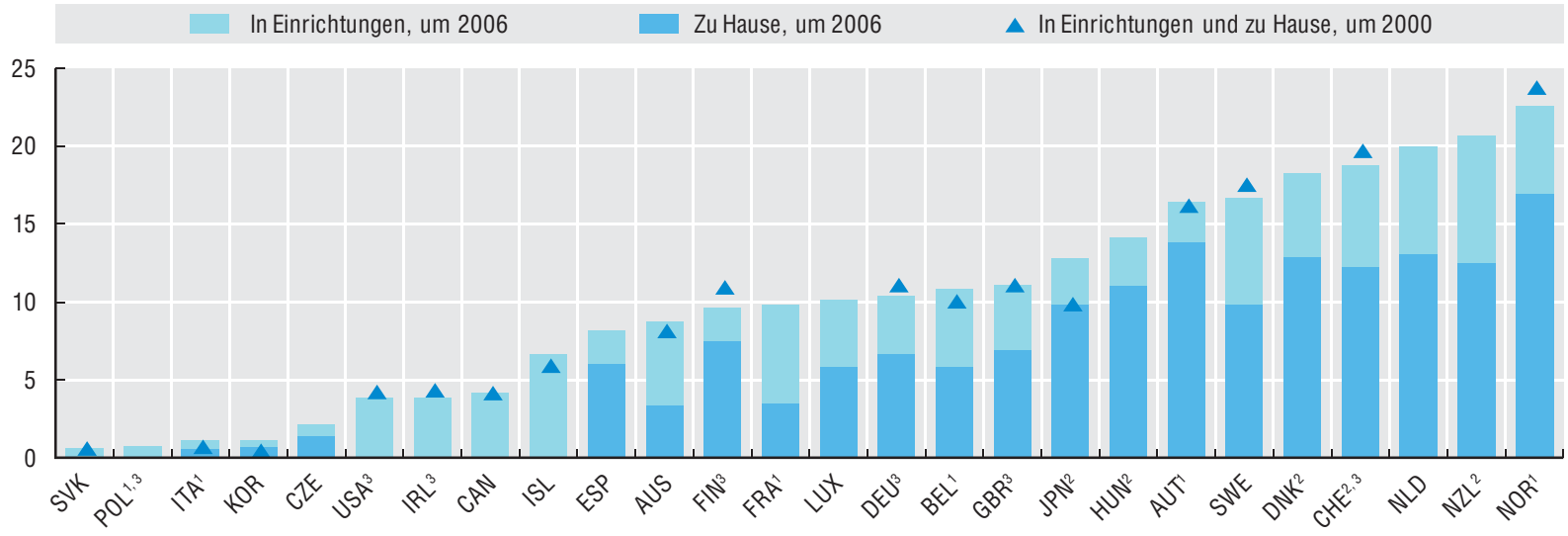

HE7.2. Der Anteil der Empfänger formeller Langzeitpflegeleistungen ist in älteren Altersgruppen höher, seit 2000 in vielen OECD-Ländern aber rückläufig

Pflegebedürftige im Alter zwischen 65 und 79 Jahren und ab 80 Jahre, als Anteil der entsprechenden Altersgruppe, 2000 und 2006

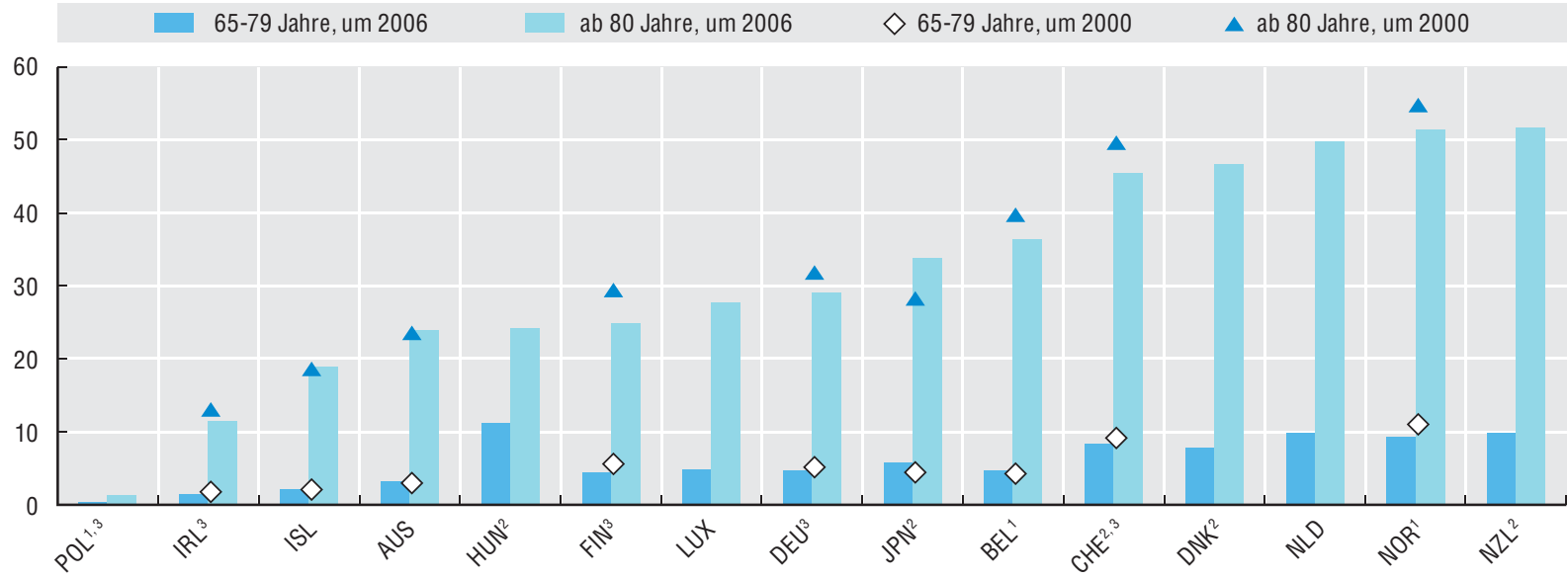

HE7.3. Mehr ältere Frauen als Männer erhalten formelle Langzeitpflege

Pflegebedürftige unter Frauen und Männern im Alter zwischen 65 und 79 Jahren und ab 80 Jahre, als Anteil der entsprechenden Altersgruppe und der Männer/Frauen insgesamt, 2006

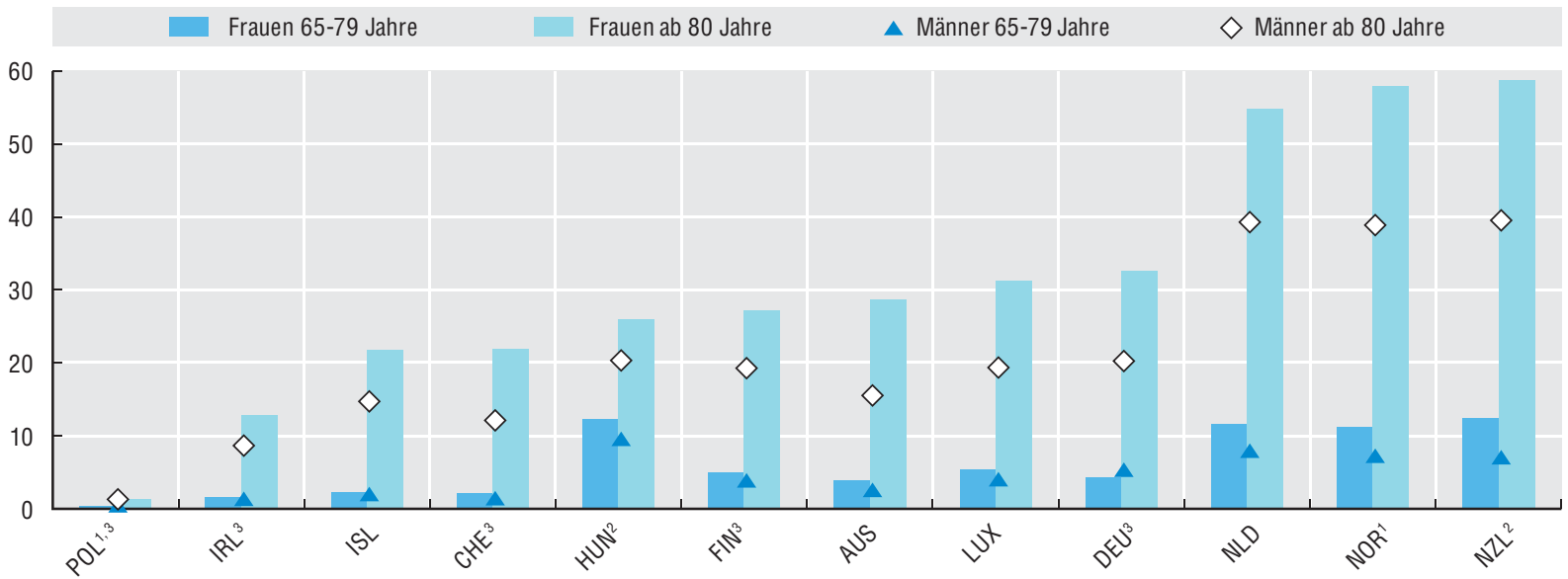

Quelle: OECD (2008), OECD-Gesundheitsdaten 2008, Paris (www.oecd.org/health/healthdata) und OECD Demographic and Labour Force database. 


\section{Definition und Messung}

Mit den Gesamtausgaben für Gesundheit werden der Endverbrauch an Gesundheitswaren und -leistungen sowie die Kapitalinvestitionen in die Gesundheitsinfrastruktur gemessen. Erfasst werden damit sowohl die öffentlichen als auch die privaten Ausgaben für die individuelle Gesundheitsversorgung ebenso wie für die Kollektivleistungen (öffentliche Gesundheits- und Vorsorgeprogramme sowie Verwaltungsleistungen). Unberücksichtigt bleiben Ausgaben im Gesundheitsbereich, die in Zusammenhang mit Ausbildung, Forschung und umweltbezogenem Gesundheitsschutz stehen.

Zum Vergleich der Gesundheitsausgaben der einzelnen Länder und im Zeitverlauf werden die Pro-KopfGesundheitsausgaben mit einem nationalen Preisindex deflationiert und auf der Basis von Kaufkraftparitäten (KKP) in US-Dollar umgerechnet.

Die durchschnittlichen Pro-Kopf-Gesundheitsausgaben variieren erheblich zwischen den einzelnen OECD-Ländern. 2006 waren die Pro-Kopf-Gesundheitsausgaben in den Vereinigten Staaten mit 6714 US-\$ am höchsten - d.h. sie beliefen sich auf mehr als das Doppelte des OECDDurchschnitts. Nach den Vereinigten Staaten kommen Norwegen, die Schweiz und Luxemburg, die alle noch weit über dem OECD-Durchschnitt liegen. Die meisten OECD-Länder befinden sich in einer Bandbreite zwischen 2400 US-\$ und 3600 US-\$. Am anderen Ende der Skala gibt eine Gruppe von vier Ländern (Türkei, Mexiko, Polen und Slowakische Republik) weniger als die Hälfte des OECD-Durchschnitts aus.

Die Veränderung bei den Niveaus der öffentlichen Gesundheitsausgaben ist mit der vergleichbar, die für die Gesamtgesundheitsausgaben zu beobachten ist. Auch Abbildung HE8.1 zeigt, dass die Gesundheitsausgaben in den Vereinigten Staaten im Verhältnis am höchsten sind, um einiges höher als in Portugal, wobei die Ausgaben in der Türkei am niedrigsten sind.

Ein wachsender Anteil an Mitteln wird für die Gesundheitsversorgung aufgewendet. Zwischen 1995 und 2006 sind die durchschnittlichen Pro-Kopf-Gesundheitsausga- ben im OECD-Raum jährlich um rd. 4\% gewachsen (HE8.2). Das durchschnittliche Wirtschaftswachstum belief sich im selben Zeitraum auf 2,5\%. Hinter diesem OECD-Durchschnitt verbergen sich aber große Unterschiede sowohl zwischen den einzelnen Ländern als auch im Zeitverlauf. Im Allgemeinen sind die Länder mit hohem Wachstum, wie Korea und Irland, diejenigen, deren Ausgangsniveau der Pro-Kopf-Gesundheitsausgaben verhältnismäßig gering war. Das Wachstum der Gesundheitsausgaben in den beiden Ländern hat in diesem Zeitraum den OECD-Durchschnitt bei weitem überstiegen. Im Gegensatz dazu verzeichneten Länder wie Deutschland und Österreich zwischen 1995 und 2006 ein relativ geringes Wachstum der Gesundheitsausgaben, was z.T. auf Kostendämpfungsmaßnahmen und ein langsames Wirtschaftswachstum zurückzuführen ist.

Reichere Länder geben mehr Geld für die Gesundheitsversorgung aus. Abbildung HE8.3 zeigt in den OECD-Ländern einen positiven Zusammenhang zwischen dem Durchschnittseinkommen und den Pro-Kopf-Gesundheitsausgaben. Das Nationaleinkommen ist nicht der einzige Faktor, der das Niveau der Gesundheitsausgaben beeinflusst. Der Zusammenhang ist in der Regel in OECD-Ländern mit niedrigerem Durchschnittseinkommen stärker. Unter Ländern mit vergleichbaren durchschnittlichen Einkommensniveaus bestehen in Bezug auf die Höhe der Gesundheitsausgaben erhebliche Unterschiede. Obwohl Japan und Deutschland z.B. ähnliche durchschnittliche Einkommensniveaus aufweisen, unterscheiden sich ihre Pro-KopfGesundheitsausgaben beträchtlich voneinander.

Die Lebenserwartung ist in Ländern höher, die mehr für die Gesundheitsversorgung ausgeben. Mit höheren ProKopf-Gesundheitsausgaben wird generell eine höhere Lebenserwartung bei der Geburt assoziiert (HE8.4), obwohl dieser Zusammenhang in Ländern mit höheren Pro-KopfGesundheitsausgaben weniger deutlich ist. Japan ragt auf Grund seiner im Verhältnis zum Niveau der Gesundheitsausgaben relativ hohen Lebenserwartung heraus, während für die Vereinigten Staaten das Gegenteil zutrifft.

\section{Anmerkungen zu den Abbildungen}

Abbildung HE8.1: Belgien und Dänemark: Bei den öffentlichen und privaten Ausgaben bleiben Investitionsausgaben unberücksichtigt. Anmerkung 1: 2005/2006. Anmerkung 2: 2005.

Abbildung HE8.2: Anmerkung 1: Brüche in den Zeitreihen. Anmerkung 2: 1999-2005. Anmerkung 3: 1997-2005. Anmerkung 4: 1995-2005. 


\section{GESUNDHEITSINDIKATOREN}

8. Gesundheitsausgaben

HE8.1. Gesundheitsausgaben als Anteil des NNE, 2006

Die Länder sind entsprechend dem NNE-Anteil der Gesundheitsausgaben aufgelistet

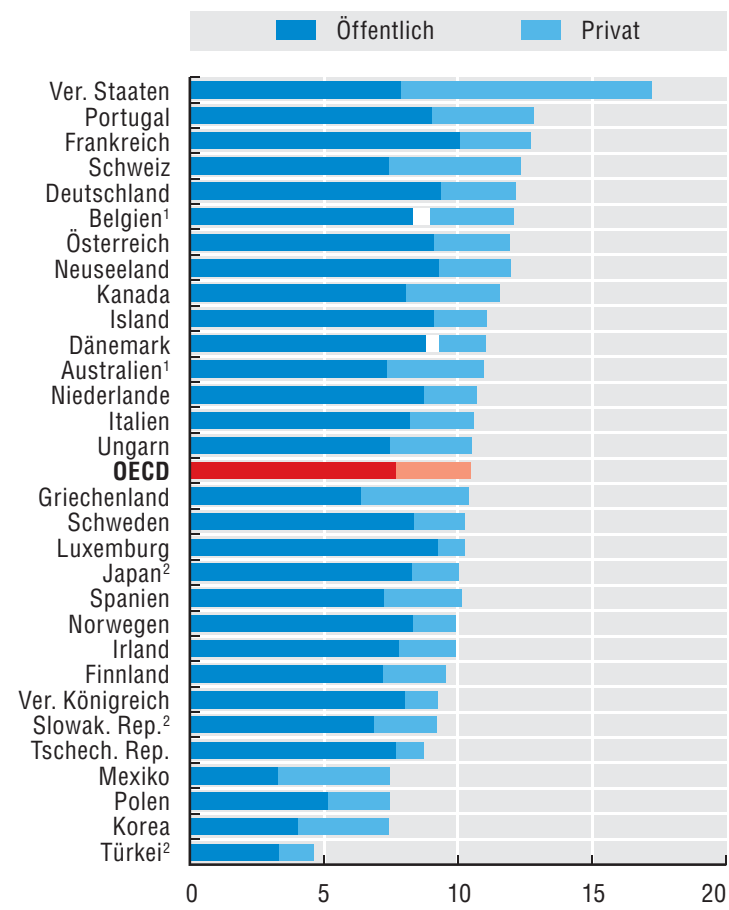

HE8.3. Reichere Länder wenden mehr Geld für die Gesundheitsversorgung je Einwohner auf, 2006

Pro-Kopf-Gesundheitsausgaben (US-\$, KKP), 2006

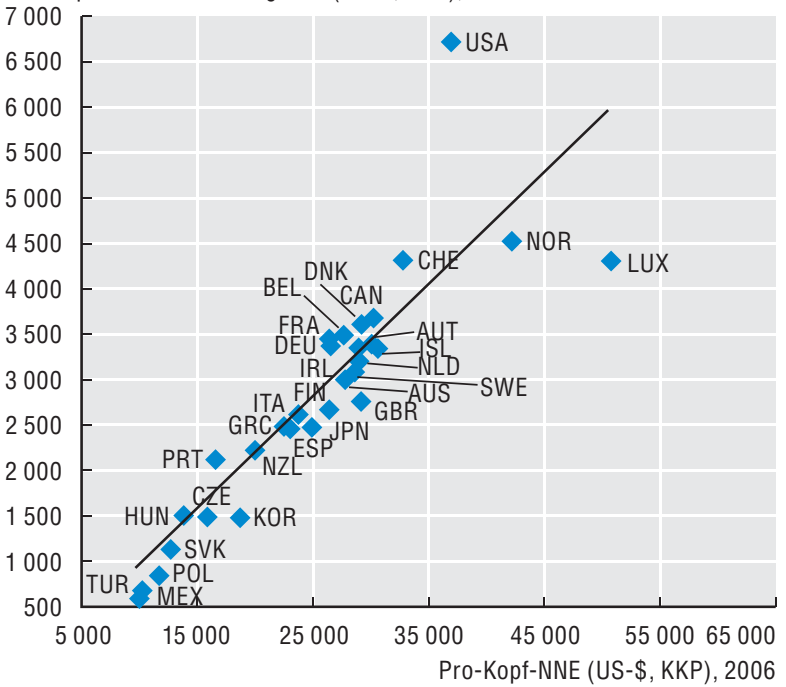

Quelle: OECD (2008), OECD-Gesundheitsdaten 2008, OECD, Paris (www.oecd.org/health/healthdata).

\section{HE8.2. Jährliches Wachstum der Pro-Kopf- Gesundheitsausgaben, 1995-2006}

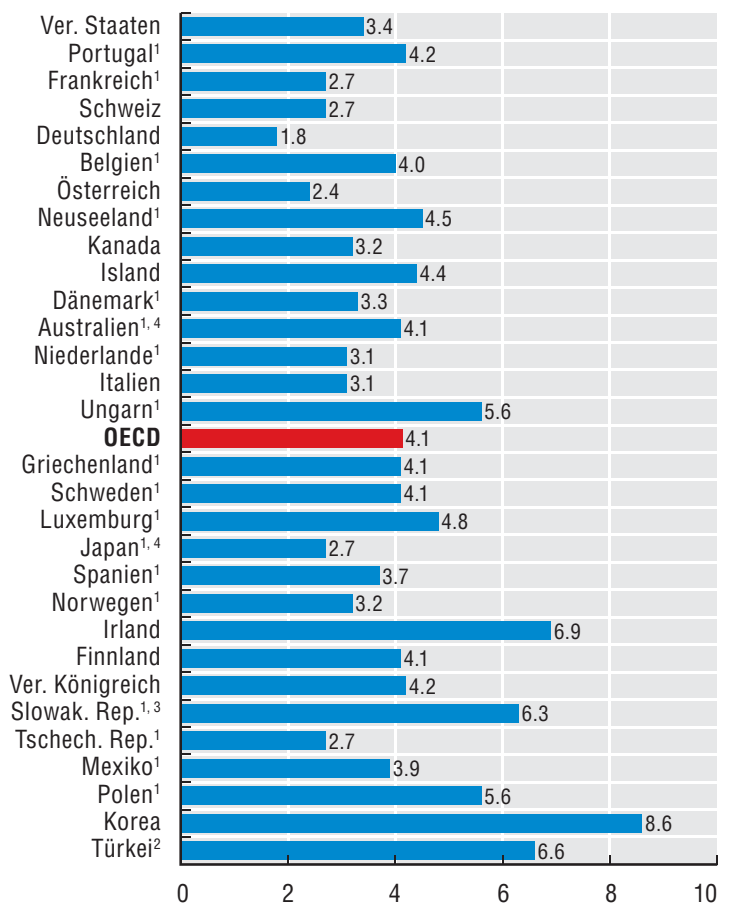

\section{HE8.4. Länder mit einer höheren Lebenserwartung geben mehr für die Gesundheitsversorgung je Einwohner aus, 2006}

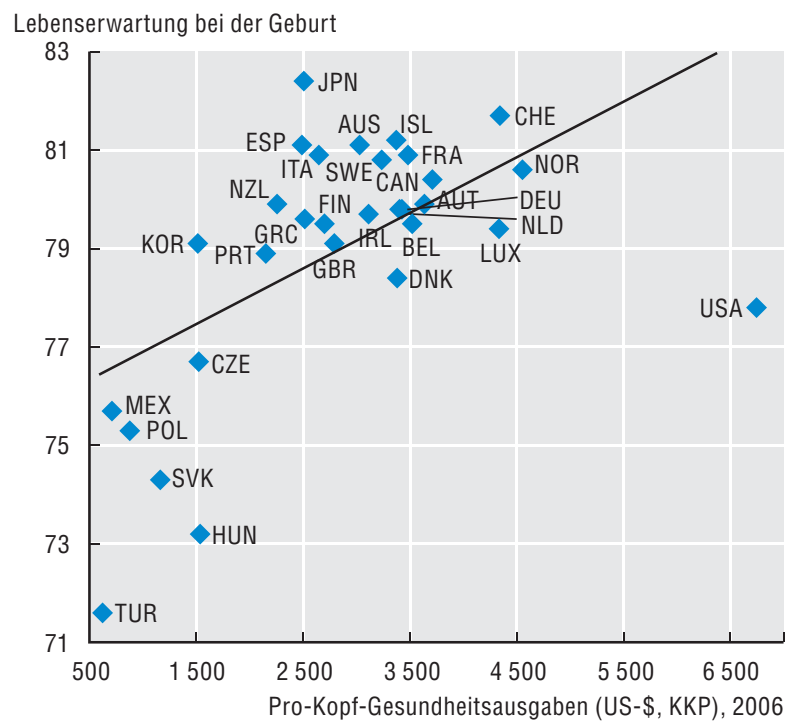




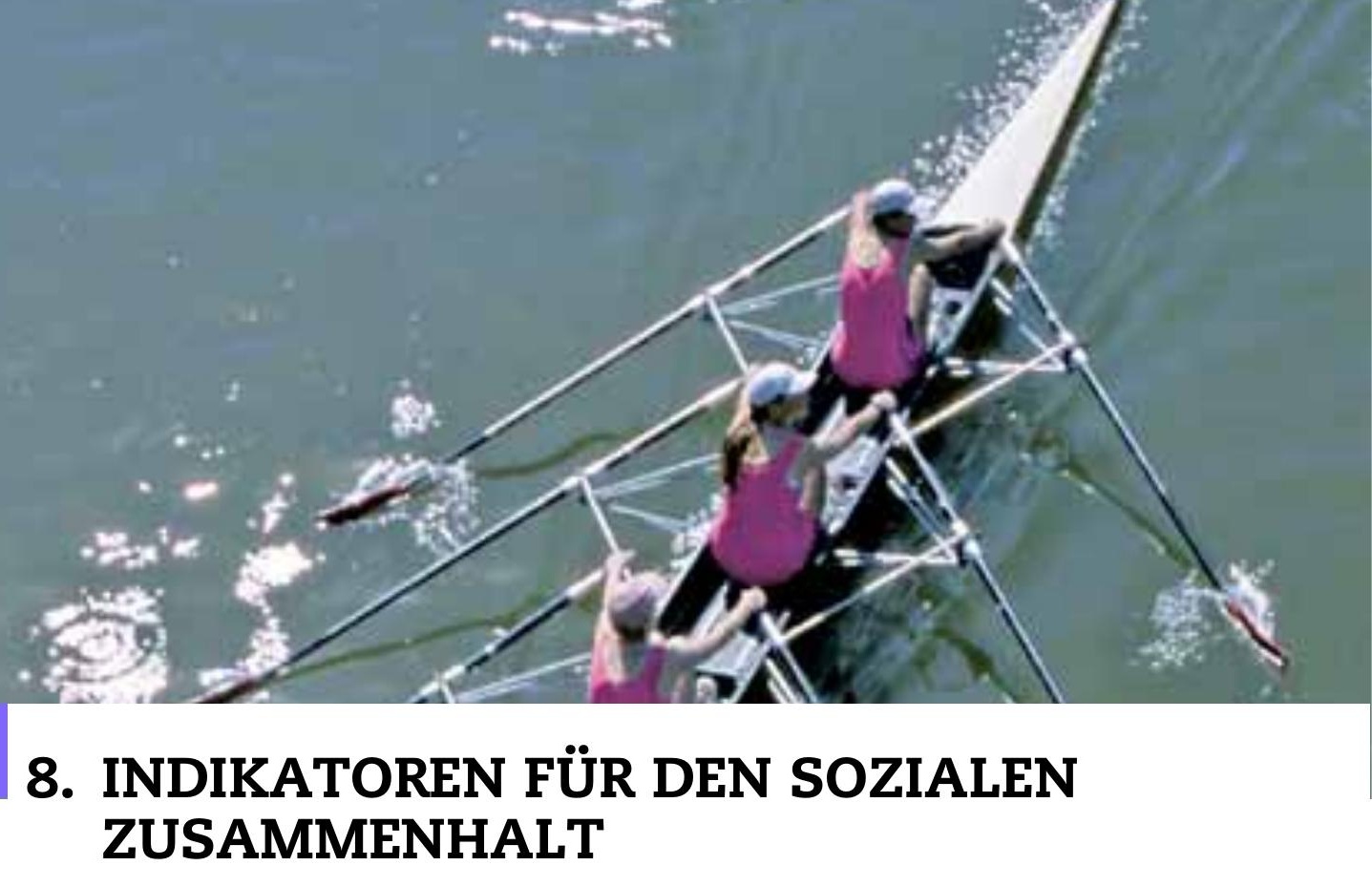

1. Lebenszufriedenheit

2. Arbeitszufriedenheit

3. Kriminalitätsbedingte Viktimisierung

4. Suizide

5. Bullying

6. Risikoverhalten 


\section{Definition und Messung}

Der Hauptindikator der Lebenszufriedenheit stammt aus der Gallup World Poll 2006. Die Gallup-Umfrage wurde bei repräsentativen nationalen Stichproben der Wohnbevölkerung ab 15 Jahre durchgeführt. In allen Ländern wird derselbe Fragebogen verwendet, um ein Höchstmaß an Vergleichbarkeit zu gewährleisten, wenngleich noch immer Unklarheit darüber besteht, inwieweit sich das in der englischen Sprache verwendete Konzept der „life satisfaction“ (Lebenszufriedenheit) in die verschiedenen im OECD-Raum gesprochenen Sprachen übertragen lässt. Dabei sind die Schwierigkeiten geringer als bei Fragen nach dem „Glücksempfinden“, was auch erklärt, warum hier Fragen zur Lebenszufriedenheit vorgezogen wurden. In der Gallup World Poll wird den Teilnehmern folgende Frage gestellt: „Stellen Sie sich eine Leiter mit elf Stufen vor, auf der die unterste Stufe (0) das für sie unerträglichste und die höchste Stufe (10) das für sie bestmögliche Leben darstellt. Auf welcher Stufe der Leiter stehen Sie Ihrem Gefühl nach mit Ihrem Leben heute?" Der in diesem Abschnitt verwendete Hauptindikator ist der Länderdurchschnitt. Der Zugang zu den nach soziodemografischen Merkmalen aufgeschlüsselten detaillierten Daten der Gallup-Umfrage war nicht möglich. Die Verlässlichkeit von Daten aus Festnetzinterviews, wie in der Gallup-Erhebung wird insbesondere in jenen Ländern in Frage gestellt, in denen das Mobilfunknetz weit verbreitet ist. Die Daten zur Messung von Veränderungen der Lebenszufriedenheit stammen aus der World Happiness-Datenbank (weltgrößte Datenbank des Glücks), die ihre Informationen aus unterschiedlichen Quellen bezieht, in erster Linie der Eurobarometer-Umfrage und der World Values Survey (vgl. Kasten 1.1 in Kapitel 1 wegen näherer Einzelheiten).

Es gibt erhebliche Unterschiede zwischen den Ländern in Bezug auf das Ausmaß, in dem die Menschen mit ihrem Leben zufrieden sind (Abbildung C01.1). Dänemark, die Schweiz und Finnland, die drei Länder mit der höchsten Lebenszufriedenheit, sind auf der 11-Stufen-Leiter 2,7 Stufen höher angesiedelt als die drei untersten Länder (Slowakische Republik, Italien und Türkei).

Es gibt große regionale und kulturelle Cluster bei der Einschätzung der Lebenszufriedenheit. Drei der sechs bestplatzierten Länder sind nordische Länder, wobei Island als nordisches Land mit einem Platz im Mittelfeld ein Aus- reißer ist. Nicht besonders zufrieden mit ihrem Leben sind die Einwohner der west- und ostkontinentaleuropäischen OECD-Länder, mit der nennenswerten Ausnahme der Schweizer und Niederländer, und in geringerem Ausmaß auch der Belgier und Spanier. Die hauptsächlich englischsprachigen OECD-Länder (Vereinigtes Königreich, Irland, Vereinigte Staaten, Neuseeland, Australien und Kanada) sind in Bezug auf die Lebenszufriedenheit alle in der oberen Hälfte angesiedelt, als geschlossene Gruppe gleich hinter dem bestplatzierten Cluster überwiegend nordeuropäischer Länder.

Die Lebenszufriedenheit ist in wohlhabenderen OECDLändern höher (Abbildung CO1.2). Es besteht ein starker Zusammenhang, der zugleich aber nicht linear zu sein scheint, was bedeuten könnte, dass der Beitrag des Einkommenszuwachses eines Landes zur Steigerung der Lebenszufriedenheit mit zunehmendem Wohlstand abnimmt. Außerdem gibt es interessante Ausreißer von der Regressionslinie. Mexiko, Neuseeland und Dänemark weisen ein sehr viel höheres Niveau der Lebenszufriedenheit auf als ihr NNE erwarten lässt, Luxemburg, Irland und die Türkei hingegen eine vergleichsweise sehr viel geringere Zufriedenheit.

Ein hoher Zufriedenheitsgrad geht häufig mit einer gerechteren Verteilung der Zufriedenheit in der Bevölkerung einher (Abbildung CO1.3). In Ländern mit geringerer durchschnittlicher Lebenszufriedenheit sind die Ungleichheiten der Lebenszufriedenheit auch innerhalb eines Landes häufig größer, wie die Standardabweichung einzelner Punktwerte zeigt. Der Zusammenhang ist stark.

Die Lebenszufriedenheit verbessert sich mit der Zeit. Abbildung CO1.4 zeigt, dass die anhand der 11-StufenLeiter gemessene Lebenszufriedenheit im OECD-Raum im Durchschnitt um 0,28 Stufen gestiegen ist. In 23 Ländern nahm die Lebenszufriedenheit zu oder blieb konstant, rückläufig war sie nur in Portugal, Ungarn, den Vereinigten Staaten, Kanada und Japan. Außergewöhnlich stark verbessert hat sich die Lebenszufriedenheit in der Türkei.

\section{Weitere Informationen}

Deaton, A. (2007), „Income, Aging, Health and Wellbeing around the World: Evidence from the Gallup World Poll“, NBER Working Paper No. 13317, Cambridge, MA.

\section{Anmerkungen zu den Abbildungen}

Abbildung C01.4: In den meisten Fällen decken die Daten zur Veränderung der Lebenszufriedenheit den Sechsjahreszeitraum 2000-2006 ab. Informationen zu Quellen und länderspezifischen Ausnahmen finden sich in Kasten 1.1 in Kapitel 1. 
C01.1. Erhebliche Unterschiede zwischen den Ländern hinsichtlich der Lebenszufriedenheit

Durchschnittliche Punktzahl der Lebenszufriedenheit auf einer 11-Stufen-Leiter von 0-10, 2006

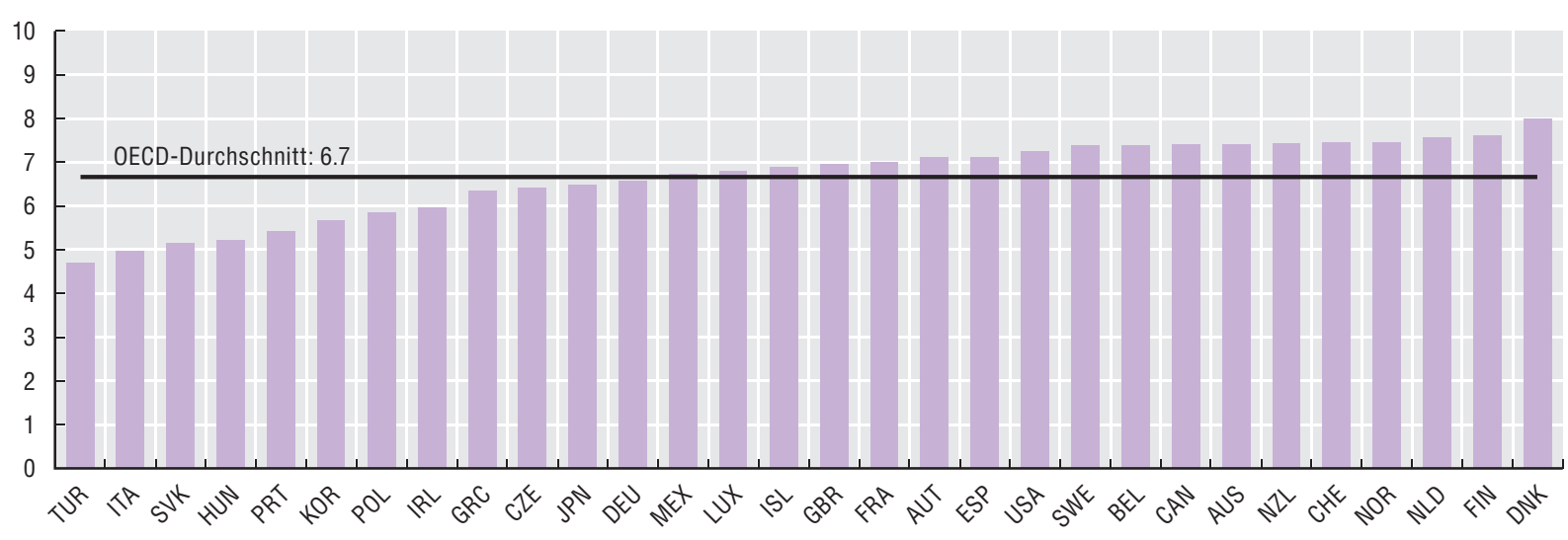

Quelle: Gallup World Poll.

\section{C01.2. Lebenszufriedenheit steigt mit höherem NNE, 2006}

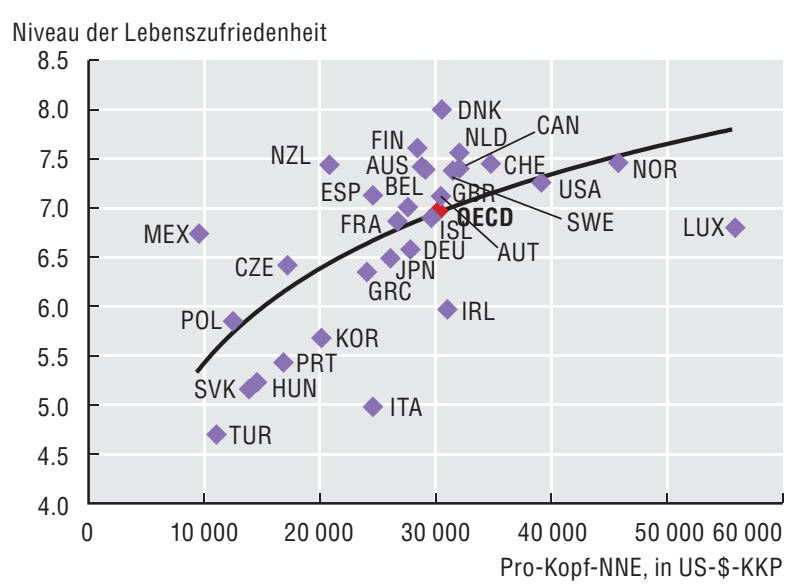

C01.3. Eine geringere Lebenszufriedenheit geht mit größeren Ungleichheiten der Lebenszufriedenheit einher, 2006

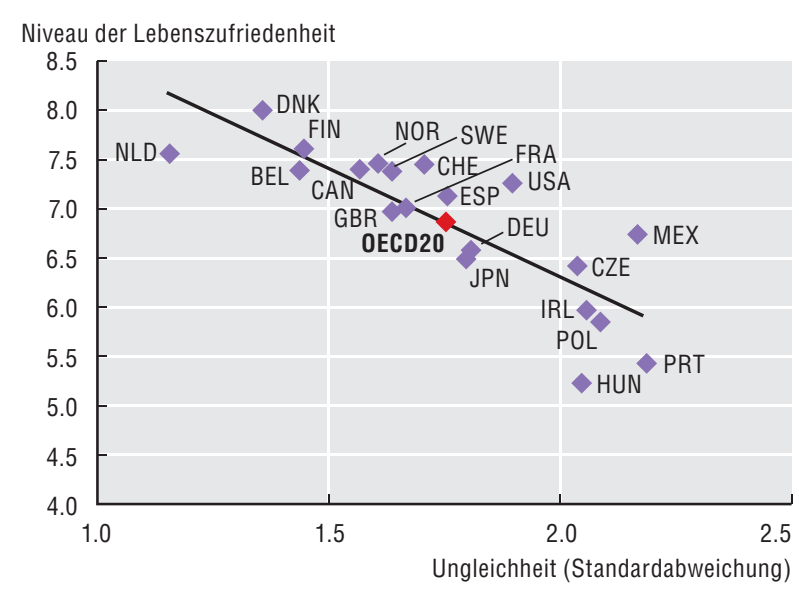

Quelle: Gallup World Poll; OECD National Accounts (www:oecd.org/statistic/national accounts).

C01.4. Die Lebenszufriedenheit steigt in den meisten OECD-Ländern

Veränderungen der Lebenszufriedenheit, Punkte auf einer 11-Stufen-Leiter, 2000-2006

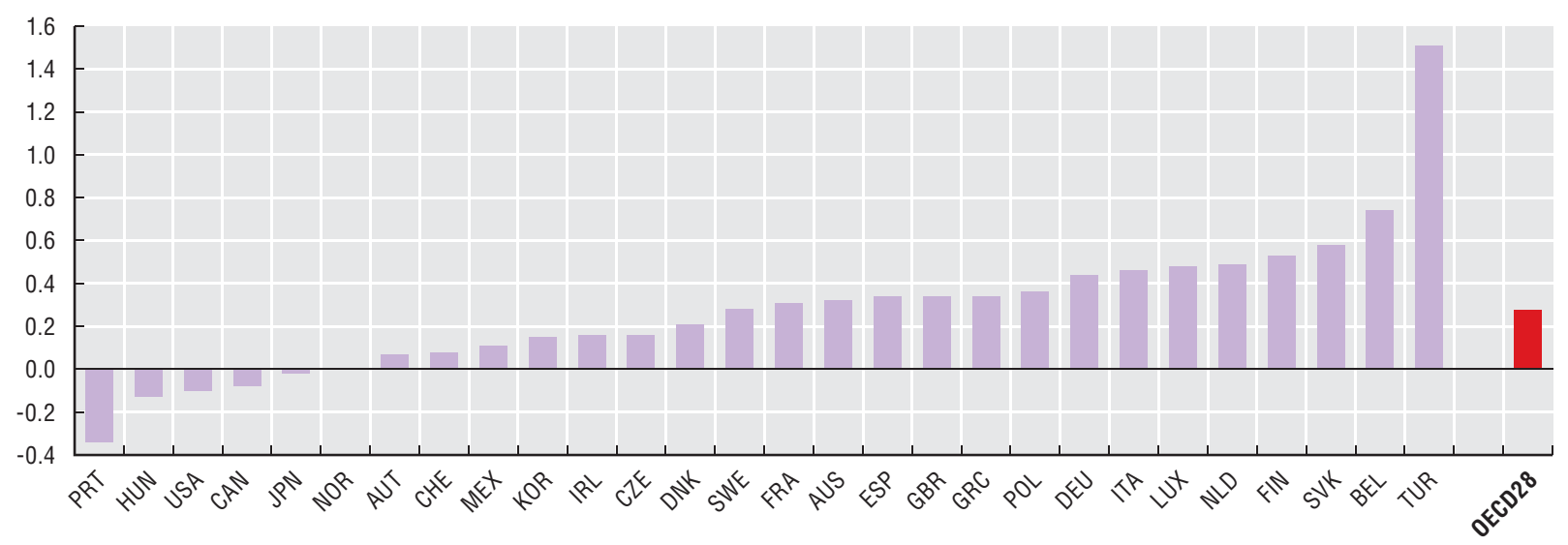




\section{Definition und Messung}

Die Messgrößen der Arbeitszufriedenheit sind der dritten Erhebungswelle des International Social Science Programme (ISSP) entnommen. Die letzte ISSP-Erhebungsrunde $\mathrm{zu}$ den Arbeitsorientierungen fand 2005 statt. Frühere Erhebungswellen wurden in den Jahren 1989 und 1997 veranstaltet. Zielgruppe der Umfrage waren Personen ab 16 Jahre, die entweder abhängig oder selbstständig beschäftigt waren. An der letzten Erhebungswelle dieser Untersuchung haben 21 OECD-Länder teilgenommen. Die generell hohen und zwischen den Ländern und im Zeitverlauf schwankenden Nonresponse-Raten sowie von Land $\mathrm{zu}$ Land unterschiedlichen Stichprobenrahmen sind Faktoren, die die Vergleichbarkeit beeinträchtigen können.

Der hier verwendete Grundindikator der Arbeitszufriedenheit ist der Anteil aller Erwerbstätigen, der angibt, in seinem Hauptberuf im Allgemeinen „völlig“, „sehr“ oder „ziemlich“ zufrieden zu sein (unter sieben Antwortkategorien). Die anderen Messgrößen betreffen die Einstellung der Befragten zu bestimmten Merkmalen der beruflichen Arbeit und des Berufs sowie zu ihrer Arbeit. Die Bedeutung, die die Teilnehmer persönlich bestimmten Merkmalen der beruflichen Arbeit und des Berufs beimessen, wird anhand ihrer Antwort auf die Frage gemessen „wie wichtig für Sie persönlich“ eine Liste von Merkmalen ist (sechs Antwortkategorien). Zur Ermittlung der Eigenschaften der aktuellen Arbeit werden die Teilnehmer gefragt, inwieweit eine Reihe von Aussagen auf ihre Arbeit zutrifft (erneut sechs Antwortkategorien). In beiden Fällen gibt es in der Fragestellung kein Ranking zwischen den Kategorien. Die Stichprobenumfänge sind mit etwa 1000 oder 2000 Befragten klein. Die Angaben für Belgien beziehen sich ausschließlich auf Flandern, die für Deutschland nur auf die westdeutschen Bundesländer, und bei den Daten für das Vereinigte Königreich ist Nordirland ausgeklammert.

Die meisten Menschen sind im Allgemeinen in ihrem Beruf zufrieden (Abbildung CO2.1). Im Durchschnitt der 21 OECD-Länder liegt der Anteil der Personen, die angeben, in ihrem Beruf völlig, sehr oder ziemlich zufrieden zu sein, bei nahezu $80 \%$. Die Zahlen reichen von etwa $70 \%$ in Korea bis zu über $90 \%$ in Mexiko und der Schweiz. Etwa 12\% der Befragten waren im Durchschnitt völlig zufrieden, gegenüber $30 \%$, die sehr, und $40 \%$, die ziemlich zufrieden waren.

Immer mehr Personen sind mit ihrem Beruf zufrieden. Seit 1997 ist der Anteil der mit ihrem Beruf zufriedenen abhängig Beschäftigten in den elf Ländern, für die Vergleiche möglich sind, um etwa 5 Punkte gestiegen. In Schweden wurde eine deutliche Zunahme verzeichnet, während in Frankreich, Spanien und Dänemark ein Rückgang beobachtet wurde (Abbildung CO2.1).

Es gibt geringe Unterschiede in der Arbeitszufriedenheit nach Geschlecht und Alter. In den 21 OECD-Ländern, die in der Erhebung von 2005 untersucht wurden, waren Frauen mit ihrer Arbeit ebenso zufrieden wie Männer. In Portugal, der Schweiz, Belgien, Korea, Deutschland, Japan und den Vereinigten Staaten sind die Frauen mit ihrer Arbeit weniger und in Irland, der Tschechischen Republik, Australien, Mexiko und Finnland stärker zufrieden als die Männer. Bis zum Alter von 65 Jahren nimmt die Arbeitszufriedenheit im Allgemeinen mit dem Alter geringfügig zu, obgleich mehrere Länder eine Ausnahme bilden (ISSP 2005).

Die Arbeitsplatzsicherheit ist das Merkmal, dem die Erwerbstätigen die größte Bedeutung beimessen. Gefolgt von einem hohen Einkommen, Aufstiegsmöglichkeiten und flexiblen Arbeitszeiten, wenngleich es in Bezug auf diese Kriterien unter den Ländern große quantitative Unterschiede gibt. (ISSP 2005).

Die meisten Menschen sind mit ihrer derzeitigen Arbeitszeit- und Einkommenskonstellation glücklich. Auf die Frage, ob sie genau so viel Stunden arbeiten und genau so viel Geld verdienen oder mehr Stunden arbeiten und mehr Geld verdienen oder weniger Stunden arbeiten und weniger Geld verdienen möchten, antwortete die Mehrzahl der Befragten in allen Ländern, mit ihrer aktuellen Situation (Option 1) zufrieden zu sein. Etwa ein Drittel der Befragten würde lieber mehr arbeiten und mehr Geld verdienen, ihr Anteil erreichte in Mexiko und Frankreich die Hälfte oder etwas mehr. In Dänemark hingegen ist die Zahl der Personen, die bevorzugen, weniger zu arbeiten (und weniger zu verdienen) größer als die der Personen, die mehr arbeiten (und mehr verdienen) möchten. In anderen nordischen Ländern und in der Schweiz sind die Unterschiede zwischen den beiden Gruppen, die sich Veränderungen wünschen, unerheblich (ISSP 2005).

\section{Weitere Informationen}

International Social Survey Programme (ISSP) (2005), Work Orientations III, Data and Documentation, www.gesis.org/en/services/data/survey-data/issp/modulesstudy-overview/work-orientations/2005/.

Vecernik, J. (2003), „Skating on Thin Ice: A Comparison of Work Values and Job Satisfaction in CEE and EU Countries", International Journal of Comparative Sociology, Vol. 44, S. 444-471, Dezember.

\section{Anmerkungen zu den Abbildungen und Tabellen}

Abbildung CO2.1: Die Länder sind von links nach rechts in aufsteigender Reihenfolge des Anteils der Personen aufgeführt, die mit ihrer Arbeit zufrieden sind.

Tabelle CO2.2: Diese Daten basieren auf einer Auswahl von Merkmalen und nicht einer Kodierung offener Antworten. 
C02.1. Hoch und steigend: Arbeitszufriedenheit im OECD-Raum

Prozentsatz aller abhängig Beschäftigten, die in ihrem Beruf völlig, sehr oder ziemlich zufrieden sind, 2005

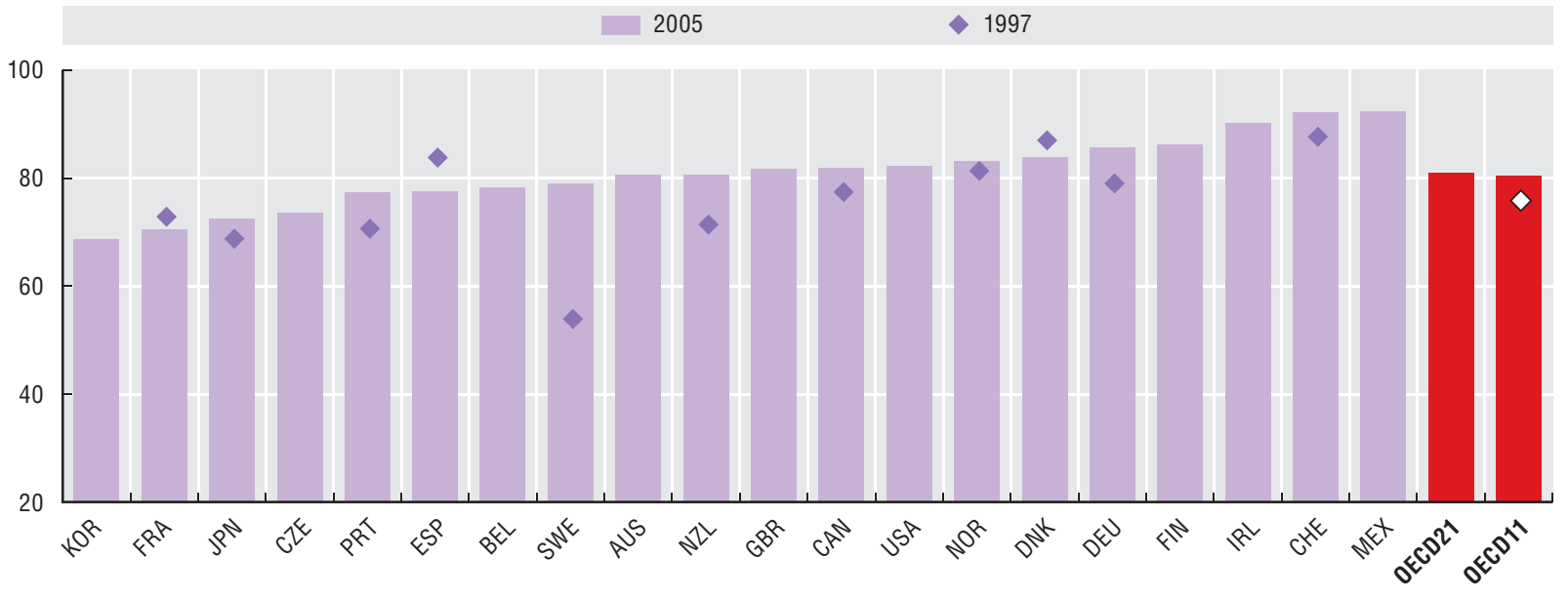

CO2.2. Von den Befragten am meisten geschätzte Berufs- und Arbeitsplatzmerkmale, 2005

\begin{tabular}{|c|c|c|c|c|c|c|c|c|c|c|c|c|c|}
\hline & \multicolumn{4}{|c|}{ Wichtige Merkmale aller Arbeitsplätze } & \multicolumn{7}{|c|}{ Merkmale des aktuellen Arbeitsplatzes } & \multicolumn{2}{|c|}{$\begin{array}{c}\text { Bevorzugte } \\
\text { Arbeitszeiten }\end{array}$} \\
\hline & \multirow[b]{2}{*}{$\begin{array}{l}\text { Arbeits- } \\
\text { platz- } \\
\text { sicherheit }\end{array}$} & \multirow[b]{2}{*}{$\begin{array}{l}\text { Hohes } \\
\text { Ein- } \\
\text { kommen }\end{array}$} & \multirow[b]{2}{*}{$\begin{array}{l}\text { Aufstiegs- } \\
\text { möglich- } \\
\text { keiten }\end{array}$} & \multirow[b]{2}{*}{$\begin{array}{r}\text { Arbeitszeit- } \\
\text { flexibilität }\end{array}$} & \multirow[b]{2}{*}{$\begin{array}{l}\text { Arbeits- } \\
\text { platz- } \\
\text { sicherheit }\end{array}$} & \multirow[b]{2}{*}{$\begin{array}{l}\text { Hohes } \\
\text { Ein- } \\
\text { kommen }\end{array}$} & \multirow[b]{2}{*}{$\begin{array}{c}\text { Aufstiegs- } \\
\text { möglich- } \\
\text { keiten }\end{array}$} & \multicolumn{4}{|c|}{ Schwierige Arbeitsbedingungen } & \multirow{2}{*}{\multicolumn{2}{|c|}{$\begin{array}{l}\text { Möchte Möchte } \\
\text { weniger mehr Zeit } \\
\text { Zeit am am Arbeits- } \\
\text { Arbeitsplatz platz } \\
\text { verbringen verbringen }\end{array}$}} \\
\hline & & & & & & & & $\begin{array}{c}\text { Nach } \\
\text { Arbeit } \\
\text { erschöpft }\end{array}$ & $\begin{array}{c}\text { Harte } \\
\text { körperliche } \\
\text { Arbeit }\end{array}$ & Stress & $\begin{array}{c}\text { Gefährliche } \\
\text { Bedingungen }\end{array}$ & & \\
\hline Australien & 95 & 71 & 83 & 55 & 64 & 24 & 25 & 90 & 45 & 85 & 29 & 67 & 33 \\
\hline Belgien & 96 & 80 & 81 & 64 & 65 & 23 & 26 & 80 & 45 & 81 & 34 & 69 & 31 \\
\hline Dänemark & 81 & 59 & 41 & 61 & 74 & 41 & 23 & 87 & 48 & 81 & 29 & 82 & 18 \\
\hline Deutschland & 96 & 77 & 76 & 54 & 71 & 25 & 23 & 86 & 46 & 81 & 28 & 39 & 61 \\
\hline Finnland & 94 & 82 & 47 & 72 & 60 & 30 & 20 & 84 & 47 & 84 & 36 & 72 & 28 \\
\hline Frankreich & 92 & 82 & 77 & 57 & 51 & 13 & 12 & 92 & 52 & 87 & 33 & 61 & 39 \\
\hline Irland & 94 & 79 & 88 & 58 & 75 & 33 & 36 & 82 & 42 & 66 & 25 & 62 & 38 \\
\hline Japan & 85 & 78 & 27 & 52 & 61 & 24 & 10 & 74 & 43 & 72 & 25 & 74 & 26 \\
\hline Kanada & 91 & 82 & 82 & 60 & 64 & 34 & 31 & 86 & 40 & 85 & 29 & 78 & 22 \\
\hline Korea & 97 & 93 & 91 & 68 & 40 & 16 & 25 & 83 & 69 & 87 & 39 & 21 & 79 \\
\hline Mexiko & 98 & 92 & 97 & 79 & 71 & 26 & 44 & 80 & 47 & 60 & 32 & 16 & 84 \\
\hline Neuseeland & 93 & 70 & 83 & 61 & 68 & 30 & 31 & 84 & 48 & 78 & 33 & 71 & 29 \\
\hline Norwegen & 93 & 70 & 52 & 61 & 62 & 18 & 13 & 86 & 42 & 86 & 33 & 67 & 33 \\
\hline Portugal & 97 & 93 & 93 & 69 & 62 & 16 & 36 & 88 & 49 & 73 & 29 & 47 & 53 \\
\hline Schweden & 93 & 74 & 49 & 70 & 65 & 19 & 21 & 86 & 51 & 89 & 33 & 83 & 17 \\
\hline Schweiz & 93 & 60 & 65 & 69 & 68 & 32 & 34 & 82 & 40 & 76 & 25 & 60 & 40 \\
\hline Spanien & 97 & 95 & 88 & 78 & 69 & 26 & 24 & 79 & 51 & 72 & 37 & 63 & 37 \\
\hline Tschech. Rep. & 94 & 86 & 57 & 54 & 51 & 17 & 14 & 88 & 48 & 62 & 32 & 78 & 22 \\
\hline Ungarn & 99 & 93 & 67 & 47 & 55 & 15 & 15 & 94 & 59 & 71 & 47 & 31 & 69 \\
\hline Ver. Königreich & 95 & 74 & 78 & 54 & 68 & 20 & 27 & 90 & 51 & 86 & 24 & 72 & 28 \\
\hline Ver. Staaten & 93 & 81 & 88 & 53 & 74 & 27 & 38 & 85 & 47 & 79 & 32 & 54 & 46 \\
\hline OECD21 & 94 & 80 & 72 & 62 & 64 & 24 & 25 & 85 & 48 & 78 & 32 & 60 & 40 \\
\hline
\end{tabular}




\section{Definition und Messung}

Die Kriminalität lässt sich auf Länderebene anhand von Erhebungen vergleichen, die darauf abzielen, Kriminalitätserfahrungen aus der Sicht der Opfer zu evaluieren. Die hier vorgestellten Kriminalitätsstatistiken entstammen dem International Crime Victim Survey (ICVS) aus dem Jahr 2005, der von einem Konsortium unter Leitung des Interregionalen Forschungsinstituts der Vereinten Nationen für Kriminalität und Rechtspflege (UNICRI) und dem Büro der Vereinten Nationen für Drogen- und Verbrechensbekämpfung erstellt wird. Die ICVS-Daten für die europäischen Länder sind dem European Survey on Crime and Safety entnommen, der von einem Konsortium unter der Leitung von Gallup Europe organisiert wird (vgl. www.europeansafetyobservatory.eu/ eucs_rp.htm wegen näherer Einzelheiten). Die zur Messung der Veränderungen zu Grunde gelegten Daten beziehen sich auf unterschiedliche Jahre.

Der ICVS konzentriert sich auf zehn Arten „konventioneller" Kriminalität (gemäß der ICVS-Kategorisierung). Die Teilnehmer werden gefragt, ob sie oder ein Mitglied ihrer Familie Opfer einer dieser konventionellen Straftaten waren. Bei diesen Verbrechen geht es um Straftaten im Zusammenhang mit Kraftfahrzeugen (Kfz-Diebstahl, Autoaufbrüche, Motorradoder Fahrraddiebstahl), Einbruchsdiebstahl und versuchten Einbruch, Diebstahl privaten Eigentums sowie Kontaktdelikte (Raub, Sexualdelikte gegen Frauen sowie Angriffe oder Bedrohungen). Sie umfassen auch die Drogenkriminalität und nichtkonventionelle Straftaten, wie Hassverbrechen, Korruption im Alltag sowie Verbraucherbetrug (insbesondere Internetbetrug und Kreditkartendiebstahl). Der ICVS enthält ferner Informationen zu Reaktionen auf unterschiedliche Verbrechensarten, zur Verbrechensfurcht sowie zum Einsatz präventiver Maßnahmen. Auch wenn die Erhebungsergebnisse sich auf repräsentative nationale Stichproben stützen, werden sie durch inhärente Merkmale wie den Befragungsmodus und die Zeitspanne der Feldarbeit beeinflusst. Die Stichprobengröße ist in der Regel gering (in den meisten Ländern 2000 Personen). Einige Delikte, wie Gewalt in der Ehe oder Sexualverbrechen, können Probleme der Vergleichbarkeit aufwerfen und eine mehr oder minder starke Untererfassung aufweisen. Ebenso kann es vorkommen, dass Menschen Opfer krimineller Taten sind, wie Betrug und Korruption, ohne dies zu merken, wodurch es natürlich wieder zu einer Untererfassung kommt.

Jede sechste Person in den OECD-Ländern war Opfer einer konventionellen Straftat (Abbildung CO3.1). 2004/2005 überstieg die Viktimisierungsrate in Irland, Neuseeland, Island und dem Vereinigten Königreich 20\%. Umgekehrt lag sie in Japan und Spanien bei unter $10 \%$.

Die Viktimisierungsraten sind seit Beginn des neuen Jahrtausends zurückgegangen, zumindest für die zehn Kategorien konventioneller Kriminalität. In $18 \mathrm{der}$ 20 OECD-Länder, für die Informationen zur Verfügung stehen, wurde ein Rückgang beobachtet. In Spanien, Italien, Australien, Schweden und Frankreich sind die Viktimisierungsraten um über 5 Punkte gesunken, während in der Schweiz und Norwegen ein leichter Anstieg verzeichnet wurde.

Weniger schwerwiegende Straftaten sind die häufigsten (Tabelle CO3.2). Im Durchschnitt aller in Tabelle CO3.2 erfassten OECD-Länder geben 3,7\% der Bevölkerung an, bereits Opfer eines Diebstahls privaten Eigentums und von Taschendiebstahl gewesen zu sein - wobei Irland ein sehr viel höheres und Japan ein sehr viel niedrigeres Niveau verzeichnen -, während 3,1\% angeben, bereits einen Fahrraddiebstahl und 2,9\% Angriffe oder Bedrohungen erfahren zu haben.

Kontaktverbrechen sind verhältnismäßig selten. Der Anteil der Befragten, die Opfer von Angriffen und Bedrohungen waren, reicht von etwa $5 \%$ oder mehr in Island, Irland und Neuseeland bis hin zu weniger als $1 \%$ in Japan, Italien und Portugal. Im Durchschnitt erklärt etwa 1\% der Bevölkerung, Opfer eines Raubs gewesen zu sein, in Mexiko und Irland ist das Niveau aber sehr viel höher. Sexualdelikte gegen Frauen wurden von ca. 1,8\% der Befragten berichtet, in Irland, den Vereinigten Staaten, Schweden und Island waren es über $3 \%$.

Unerwartet sind nichtkonventionelle Verbrechen geläufiger als konventionelle Verbrechen. Im Durchschnitt geben $11 \%$ der Befragten an, die eine oder andere Form von Verbraucherbetrug erfahren $\mathrm{zu}$ haben, wobei die Bandbreite von fast $25 \%$ in Griechenland bis zu weniger als $2 \%$ in Japan reicht. Der Anteil der Menschen, die von einer persönlichen Erfahrung mit Korruption berichteten, ist durchschnittlich niedrig, in Griechenland und Mexiko jedoch wesentlich höher als in anderen OECD-Ländern.

\section{Weitere Informationen}

Van Djik J., J. Van Kesteren und P. Smit Paul (2008), „Criminal Victimisation in International Perspective - Key Findings from the 2004-2005 International Crime Victims Survey and European Survey on Crime and Safety“, WODC Publication No. 257, Januar.

\section{Anmerkungen zu den Abbildungen und Tabellen}

Abbildung CO3.1: 1996 für Österreich, 1992 für Italien und Neuseeland und 1989 für Norwegen, Deutschland und Spanien.

Tabelle CO3.2: Die Raten zu den Sexualdelikten gegen Frauen sind in Prozent der Frauenbevölkerung ausgedrückt. 
CO3.1. Die konventionelle Kriminalität ist im OECD-Raum rückläufig, 2000 bis 2004-2005

Prozentsatz der Personen, die angeben, in den letzten 12 Monaten zumindest eine der 10 Kategorien von Kriminalität erfahren zu haben

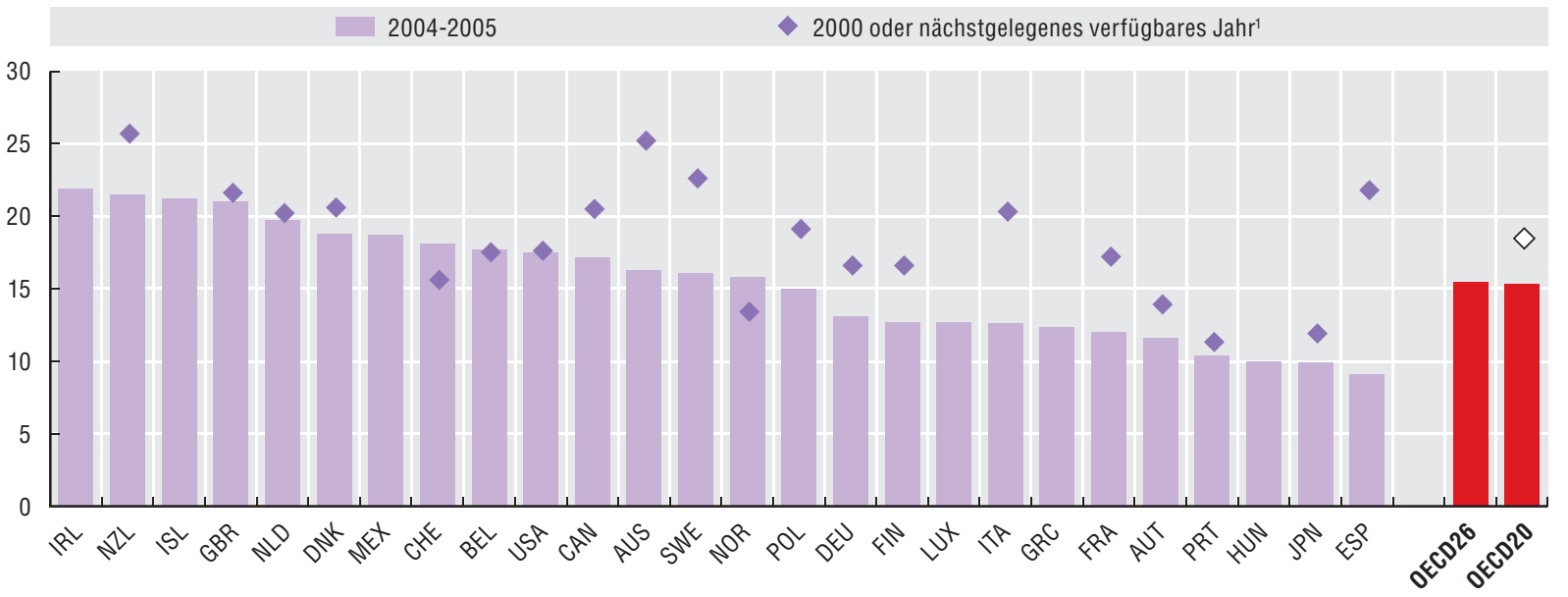

C03.2. Erfahrungen mit unterschiedlichen Verbrechenstypen und Verbrechensfurcht

Prozentsatz der Personen, die angeben, in den letzten 12 Monaten Opfer eines Verbrechens gewesen zu sein, 2004-2005

\begin{tabular}{|c|c|c|c|c|c|c|c|c|c|c|c|c|c|c|}
\hline & \multirow{4}{*}{$\begin{array}{c}\text { Konven- } \\
\text { tionelle } \\
\text { Viktimi- } \\
\text { sierung } \\
\text { insgesamt }\end{array}$} & \multicolumn{12}{|c|}{ Viktimisierung durch: } & \multirow{4}{*}{ 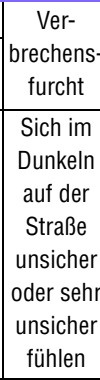 } \\
\hline & & \multicolumn{10}{|c|}{ Konventionelle Verbrechen } & \multicolumn{2}{|c|}{$\begin{array}{l}\text { Nichtkonventionelle } \\
\text { Verbrechen }\end{array}$} & \\
\hline & & \multicolumn{4}{|c|}{ Kfz-Delikte } & \multicolumn{3}{|c|}{ Einbruch und sonst. Diebstahl } & \multicolumn{3}{|c|}{ Kontaktkriminalität } & \multirow[b]{2}{*}{$\begin{array}{c}\text { Ver- } \\
\text { braucher- } \\
\text { betrug }\end{array}$} & \multirow{2}{*}{ Korruption } & \\
\hline & & $\begin{array}{c}\text { Kfz- } \\
\text { Diebstahl }\end{array}$ & $\begin{array}{c}\text { Auto- } \\
\text { aufbrüche }\end{array}$ & $\begin{array}{l}\text { Motorrad- } \\
\text { diebstahl }\end{array}$ & $\begin{array}{l}\text { Fahrrad- } \\
\text { diebstahl }\end{array}$ & $\begin{array}{l}\text { Einbruchs- } \\
\text { diebstahl }\end{array}$ & $\begin{array}{c}\text {-Versuchter } \\
\text { Einbruch }\end{array}$ & $\begin{array}{c}\text { Diebstahl } \\
\text { privaten } \\
\text { r Eigentums } \\
\text { und } \\
\text { Taschen- } \\
\text { diebstahl }\end{array}$ & Raub & $\begin{array}{c}\text { Sexual- } \\
\text { delikte } \\
\text { gegen } \\
\text { Frauen }\end{array}$ & $\begin{array}{c}\text { Angriffe } \\
\text { oder } \\
\text { Bedrohun- } \\
\text { gen }\end{array}$ & & & \\
\hline Australien & 16.3 & 1.1 & 4.5 & 0.1 & 1.2 & 2.5 & 2.4 & 3.6 & 0.9 & .. & 3.4 & & .. & 27.0 \\
\hline Belgien & 17.7 & 0.5 & 4.2 & 0.1 & 4.2 & 1.8 & 2.4 & 3.4 & 1.2 & 0.9 & 3.6 & 8.0 & 0.5 & 26.0 \\
\hline Dänemark & 18.8 & 1.3 & 2.6 & 0.3 & 6.0 & 2.7 & 1.6 & 3.3 & 0.9 & 1.9 & 3.3 & 15.7 & 1.0 & 17.0 \\
\hline Deutschland & 13.1 & 0.2 & 2.0 & 0.2 & 3.4 & 0.9 & 1.3 & 3.0 & 0.4 & 2.4 & 2.7 & 11.7 & 0.6 & 30.0 \\
\hline Finnland & 12.7 & 0.4 & 2.2 & 0.1 & 5.2 & 0.8 & 0.5 & 2.3 & 0.3 & 1.4 & 2.2 & 5.2 & 0.0 & 14.0 \\
\hline Frankreich & 12.0 & 0.6 & 3.2 & 0.3 & 0.9 & 1.6 & 1.2 & 3.3 & 0.8 & 0.4 & 2.1 & 10.2 & 1.1 & 21.0 \\
\hline Griechenland & 12.3 & 0.3 & 1.8 & 0.6 & 2.1 & 1.8 & 1.7 & 5.3 & 1.4 & 1.7 & 2.4 & 24.7 & 13.5 & 42.0 \\
\hline Irland & 21.9 & 1.2 & 5.2 & 0.3 & 2.5 & 2.3 & 1.7 & 7.2 & 2.2 & 3.8 & 4.9 & 8.0 & 0.3 & 27.0 \\
\hline Island & 21.2 & 1.0 & 3.8 & 0.1 & 4.6 & 1.6 & 1.6 & 6.9 & 0.8 & 3.0 & 5.9 & 12.9 & 0.3 & 6.0 \\
\hline Italien & 12.6 & 1.0 & 2.4 & 1.0 & 2.1 & 2.1 & 2.5 & 2.4 & 0.3 & 0.7 & 0.8 & 5.9 & 0.4 & 35.0 \\
\hline Japan & 9.9 & 0.1 & 1.1 & 0.7 & 5.1 & 0.9 & 0.7 & 0.3 & 0.2 & 1.3 & 0.6 & 1.9 & 0.2 & 35.0 \\
\hline Kanada & 17.2 & 0.8 & 4.8 & 0.2 & 2.7 & 2.0 & 1.7 & 4.0 & 0.8 & 2.3 & 3.0 & 7.4 & 0.6 & 17.0 \\
\hline Korea & & & & & & & & & & & & & & 0.0 \\
\hline Luxemburg & 12.7 & 0.6 & 2.8 & 0.0 & 1.6 & 1.7 & 2.7 & 2.9 & 0.7 & 0.6 & 2.3 & 9.8 & 0.4 & 36.0 \\
\hline Mexiko & 18.7 & 0.9 & 4.1 & 0.0 & 3.7 & 3.0 & 3.0 & 4.3 & 3.0 & 1.5 & 2.2 & 7.2 & 13.3 & 34.0 \\
\hline Neuseeland & 21.5 & 1.8 & 6.6 & 0.1 & 1.4 & 3.2 & 3.1 & 4.1 & 1.1 & 2.5 & 4.9 & 7.7 & 0.5 & 30.0 \\
\hline Niederlande & 19.7 & 1.0 & 3.9 & 0.4 & 6.6 & 1.3 & 1.4 & 3.7 & 0.5 & 1.9 & 4.3 & 7.0 & 0.2 & 18.0 \\
\hline Norwegen & 15.8 & 0.7 & 2.6 & 0.3 & 4.2 & 1.2 & 0.9 & 4.8 & 0.8 & 2.5 & 2.9 & 9.7 & 0.4 & 14.0 \\
\hline Österreich & 11.6 & 0.1 & 2.4 & 0.0 & 2.0 & 0.9 & 1.4 & 3.4 & 0.4 & 2.2 & 1.8 & 8.1 & 0.6 & 19.0 \\
\hline Polen & 15.0 & 0.7 & 3.9 & 0.1 & 2.6 & 1.4 & 1.1 & 3.5 & 1.3 & 1.3 & 3.0 & 16.1 & 4.4 & 33.0 \\
\hline Portugal & 10.4 & 1.5 & 5.0 & 0.0 & 0.5 & 1.4 & 0.8 & 1.6 & 1.0 & 0.5 & 0.9 & 8.2 & 1.0 & 34.0 \\
\hline Schweden & 16.1 & 0.5 & 4.2 & 0.6 & 5.0 & 0.7 & 0.1 & 2.4 & 1.1 & 3.3 & 3.5 & 13.7 & 0.1 & 19.0 \\
\hline Schweiz & 18.1 & 0.2 & 2.9 & 0.6 & 4.6 & 1.6 & 1.2 & 5.9 & 0.8 & 2.9 & 2.5 & 7.3 & 0.5 & .. \\
\hline Spanien & 9.1 & 1.0 & 2.7 & 0.3 & 0.7 & 0.8 & 0.4 & 2.1 & 1.3 & 0.3 & 1.6 & 10.8 & 0.3 & 33.0 \\
\hline Ungarn & 10.0 & 0.2 & 2.1 & 0.0 & 1.7 & 1.7 & 0.8 & 3.0 & 0.9 & 0.1 & 1.2 & 19.7 & 4.9 & 26.0 \\
\hline Ver. Königreich & 21.0 & 1.8 & 5.8 & 0.7 & 2.7 & 3.3 & 2.6 & 5.7 & 1.3 & 1.9 & 5.4 & .. & .. & 31.0 \\
\hline Ver. Staaten & 17.5 & 1.1 & 5.2 & 0.0 & 2.9 & 2.5 & 2.6 & 4.8 & 0.6 & 3.6 & 4.3 & 12.5 & 0.5 & 19.0 \\
\hline 0ECD23 & 15.5 & 0.8 & 3.5 & 0.3 & 3.1 & 1.8 & 1.6 & 3.7 & 1.0 & 1.8 & 2.9 & 10.4 & 1.9 & 25.7 \\
\hline
\end{tabular}

Quelle: Van Djik J., J. Van Kesteren und P. Smit Paul (2008), „Criminal

Victimisation in International Perspective - Key Findings from the 2004-2005

International Crime Victims Survey and Europen Survey on Crime and Safety“,

WODC Publication $n^{\circ} 257$, Januar. 


\section{Definition und Messung}

Die Daten zu den Suizidraten basieren auf amtlichen Statistiken über Todesursachen. Zur Bereinigung um den Effekt von Abweichungen in den Altersstrukturen zwischen den Ländern und im Zeitverlauf sind sie anhand der OECD-Bevölkerungsstruktur von 1980 standardisiert. Die Suizidraten sind in Todesfällen je 100000 Einwohner ausgedrückt.

Die Länder wenden bei der statistischen Erfassung des Suizids als zu Grunde liegende Todesursache trotz der Internationalen Statistischen Klassifikation der Krankheiten (ICD) unterschiedliche Verfahren an, die sich im Laufe der Zeit u.U. geändert haben. Die Zahl der Fälle könnte zudem auf Grund einer Stigmatisierung des Suizids durch die Gesellschaft zu niedrig ausgewiesen sein. Die soziokulturelle Norm kann von Land zu Land und im Zeitverlauf unterschiedlich sein.

Studien, in denen die Verlässlichkeit der Suizidstatistiken beurteilt wird, lassen vermuten, dass die Fehlerquellen nicht systematisch sind und sich daher auf die Vergleichbarkeit der Daten zwischen Ländern, Bevölkerungsgruppen oder im Zeitverlauf kaum auswirken (Sainsbury und Jenkins, 1982).

Die Suizidraten erhöhten sich in den 1970er Jahren und erreichten zu Beginn der 1980er Jahre einen Höchststand (Abbildung CO4.1). Zwar folgen die Suizidraten der meisten Länder diesem allgemeinen Muster, doch trifft es auf Japan, Korea und Irland nicht zu. In Japan sind die Suizidraten heute etwas niedriger als 1960, verharren aber seit 1997 auf relativ hohem Niveau (rd. 20 Suizide je 100000 Einwohner). In Korea haben die Suizide gegenüber Ende der 1990er Jahre drastisch zugenommen. Korea verzeichnet mittlerweile unter den OECD-Ländern die höchste Suizidrate (rd. 22 Todesfälle je 100000 Einwohner). Irland weist einen kontinuierlichen starken Anstieg auf, der im Jahr 2000 einen Höhepunkt erreichte, dem seither ein leichter, aber stetiger Rückgang folgte.

Die Suizidraten sind für Männer und Frauen gesunken, wobei der Abstand zwischen beiden recht stabil blieb. Da die Suizidraten für Männer und Frauen in ähnlichem Maße zurückgegangen sind, verharrt der Abstand zwischen den Geschlechtern etwa auf dem bisherigen Niveau, wobei der Suizid nach wie vor ein überwiegend männliches Phänomen ist. Im Durchschnitt entfallen auf jeden Suizid von
Frauen 3 Suizide von Männern. Allerdings gibt es beim Abstand zwischen den Suizidraten von Frauen und Männern deutliche Unterschiede. Besonders groß ist der Abstand zwischen beiden Raten nach wie vor in Mexiko, Polen und der Slowakischen Republik, wo auf jeden Suizid von Frauen mindestens 5 Suizide von Männern entfallen. Demgegenüber sind die Abstände zwischen den Geschlechtern in Korea, den Niederlanden und Norwegen geringer, wo auf einen Suizid von Frauen zwei Suizide von Männern kommen.

Ältere Menschen nehmen sich im Allgemeinen leichter das Leben, dieses Muster trifft aber nicht auf alle OECDLänder zu. Griechenland, Italien, Portugal und Korea sind Beispiele für Länder, in denen ältere Menschen ihrem Leben häufiger ein Ende setzen als junge Menschen (Abbildung CO4.3). Korea verzeichnet den am stärksten steigenden Altersgradienten. In diesem Land zeigt die Suizidkurve nach Alter ab der Altersgruppe der 45- bis 54Jährigen einen steilen Anstieg. Außerdem sind die Raten in der ältesten Gruppe (ab 75 Jahre) um mehr als das 10-Fache höher als in der Gruppe der jungen Menschen im Alter von 15-24 Jahren. Der in den letzten Jahren in Korea bei den Suiziden beobachtete Aufwärtstrend erklärt sich z.T. aus dem starken Anstieg der Suizidraten unter älteren Menschen. In einer Minderzahl von OECD-Ländern hingegen wie beispielsweise Neuseeland und Norwegen - neigen junge Menschen eher zum Freitod als die ältere Bevölkerung.

Der Abstand zwischen den Suizidraten von Männern und Frauen vergrößert sich im Allgemeinen mit dem Alter. Im Durchschnitt der OECD-Länder ist die Suizidrate in der Altersgruppe der 15- bis 19-jährigen Männer z.B. 2,7-mal so hoch wie die der Frauen in derselben Altersgruppe, die der Männer über 75 Jahre indessen 5,3-mal so hoch wie die der Frauen (Abbildung CO4.4). Dieses Muster erklärt sich möglicherweise aus der stärkeren sozialen Isolation, der sich ältere Männer nach Auflösung einer langen Partnerschaft durch Trennung oder Verlust des Partners im Vergleich zu älteren Frauen gegenübersehen.

\section{Weitere Informationen}

Sainsbury, P. und J.S. Jenkins (1982), „The Accuracy of Officially Reported Suicide Statistics for Purposes of Epidemiological Research“, Journal of Epidemiology and Community Health, Vol. 36, S. 43-48.

\section{Anmerkungen zu den Abbildungen}

Abbildung CO4.1-CO4.4: 2004 für Kanada, Deutschland, die Niederlande und Schweden; 2003 für Australien, Italien und Portugal; 2001 für Dänemark; 1997 für Belgien. 
8. INDIKATOREN FÜR DEN SOZIALEN ZUSAMMENHALT

4. Suizide

\section{C04.1. Sinkende Suizidraten in den meisten OECD-Ländern}

Suizide je 100000 Einwohner nach Altersgruppen, 2005

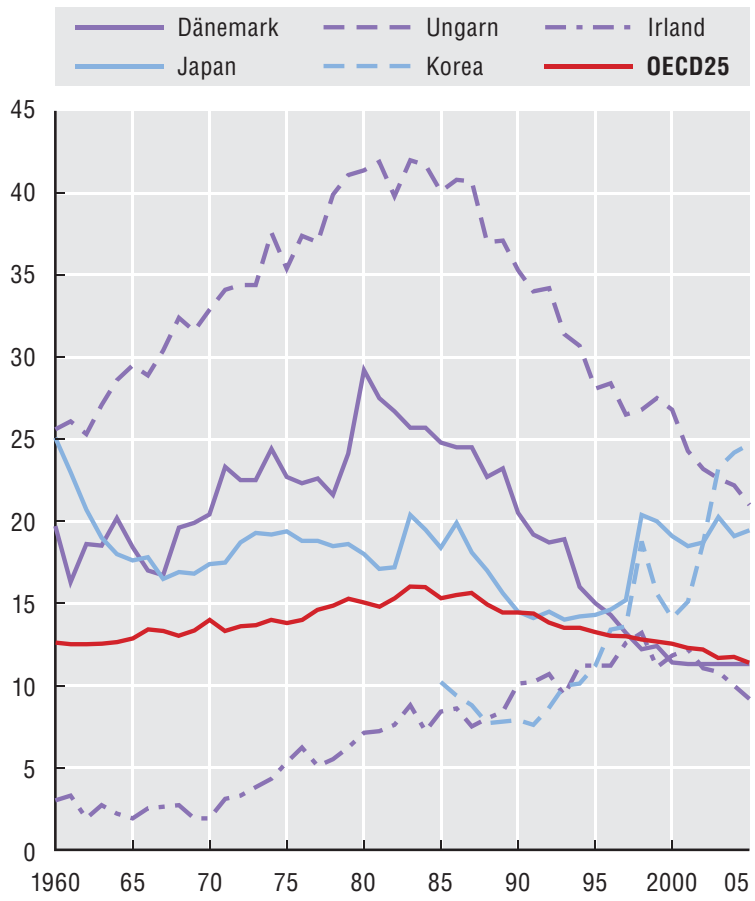

C04.3. Suizide nach Altersgruppen variieren von Land zu Land

Suizide je 100000 Einwohner nach Altersgruppen, 2005
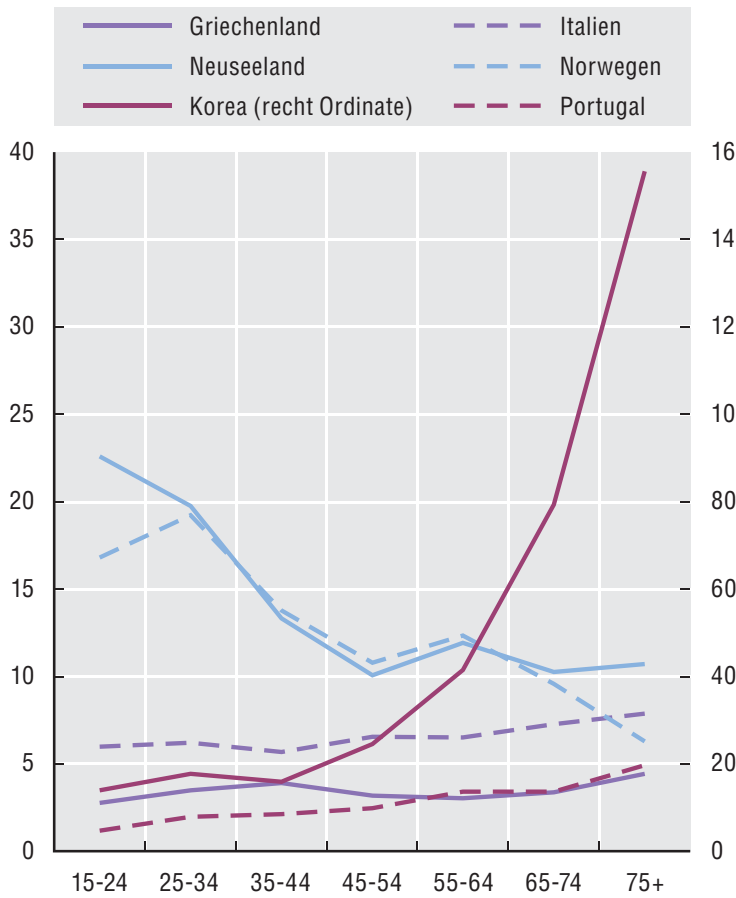

Quelle: Suicides from WHO Mortality Database (www.who.int/ healthinfo/morttables/en/index.html).
C04.2. Höhere Suizidraten bei Männern als bei Frauen Suizide je 100000 Einwohner nach Ländern und Geschlecht, 2005

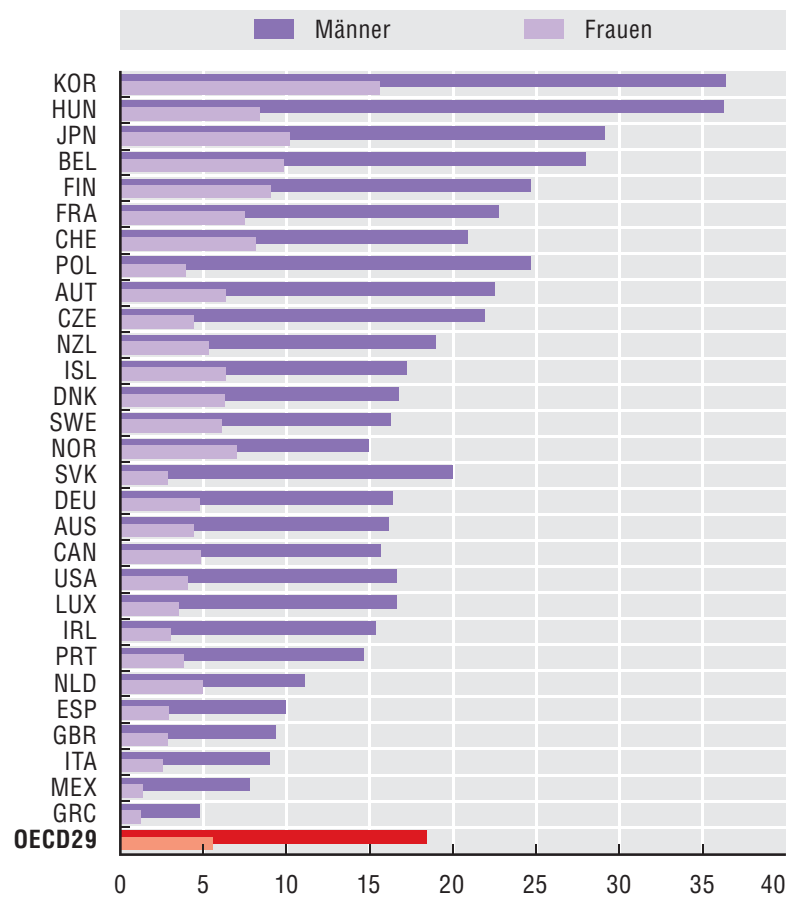

CO4.4. Genderunterschiede bei den Suizidraten in der älteren Bevölkerung sind größer

Verhältnis zwischen den Suizidraten von Männern und Frauen in ausgewählten Altersgruppen, 2005
15-19 J.
35-44 J.
ab $75 \mathrm{~J}$.

Verhältnis Suizide Männer/Frauen

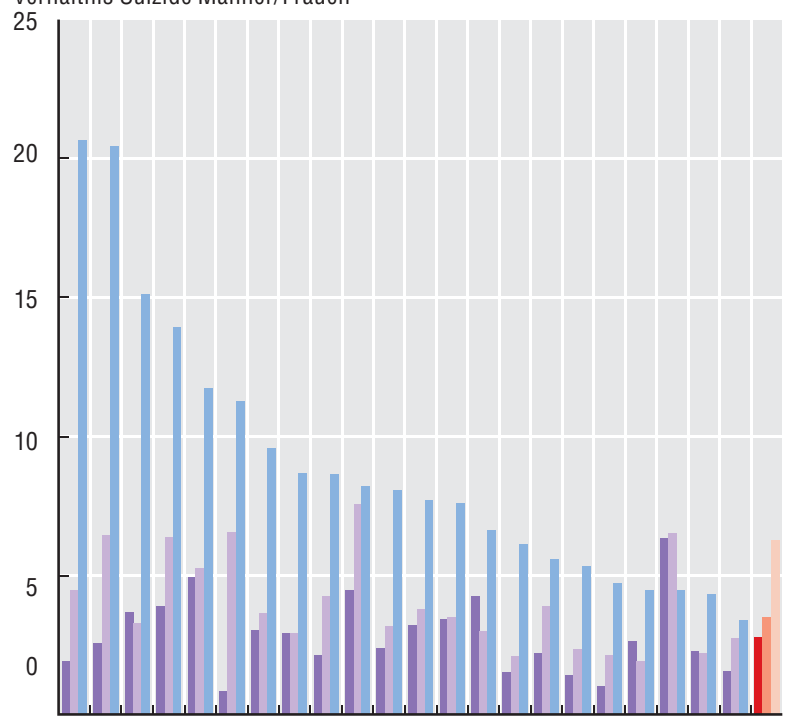

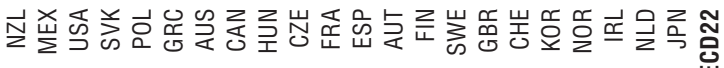




\section{Definition und Messung}

Unter Bullying versteht man wiederholte physische und psychische Aggressionen unter Schülern, aber auch passivere Formen der Viktimisierung, wie das Ausschließen von Gesprächen und Spielen. Raufereien unter gleich starken Kindern fallen nicht unter das Bullying. Die allgemeine Definition des Bullying lässt weder erkennen, welche Formen in welchem Land am weitesten verbreitet sind, noch liefert sie Aufschlüsse über Dauer und Intensität der Aggressionen.

Die Daten sind Schulstichproben aus dem Health Behaviour in School-aged Children Survey (Internationale Studie zum Gesundheitsverhalten von Schülerinnen und Schülern) für 2005/2006 entnommen. Berechnet werden die Schätzungen über die Bullying-Häufigkeit anhand der gemeldeten Täter- und Opferquoten unter den Schülern, gewichtet um die Stichprobengröße für 11-, 13- und 15-jährige Jungen und Mädchen. Die Anteile der 15-jährigen Migrantenschüler der ersten Generation basieren auf den von den Geburtsländern selbst übermittelten Statistiken, die im Rahmen der Ergebnisse von PISA 2006 veröffentlicht wurden.

\begin{abstract}
Aktives und passives Bullying sind keine Seltenheit. Etwa jedes zehnte Kind im OECD-Raum war in letzter Zeit ein Bullying-Opfer, und der Anteil der Täter ist ähnlich groß. Abbildung CO5.1 zeigt, dass die Gefahr, schikaniert zu werden, in der Türkei und Griechenland am größten ist. Am wenigsten verbreitet ist das Phänomen in Schweden und Spanien. Griechenland und Österreich zählen die meisten Bullies, während ihre Zahl in Schweden, der Tschechischen Republik und Island am niedrigsten ist.
\end{abstract}

Die Täter und Opfer sind häufiger Jungen als Mädchen. Nur in Ungarn und Griechenland sind Mädchen häufiger oder genauso häufig Bullying-Opfer wie Jungen. Es gibt kein Land, in dem mehr Mädchen als Jungen Täter sind.

Die Zahl der Täter übersteigt geringfügig die der Opfer, was darauf hindeutet, dass die Täter in Gruppen agieren. Forschungsbeobachtungen des Bullying-Phänomens haben ergeben, dass bei $80-90 \%$ aller Bullying-Prozesse nicht nur Täter und Opfer, sondern auch andere Kinder beteiligt sind: Drei Viertel dieser Kinder bestätigen das Bullying-Verhalten bzw. unternehmen zumindest nichts dagegen (Atlas et al., 1998, und Hawkins et al., 2001). Unter den Jungen besteht auf Länderebene kein klarer Zusammenhang zwischen der Zahl der Täter und der Zahl der Opfer. Für die Mädchen ergibt sich ein etwas klareres Bild, da mehr Mädchen schikaniert werden als schikanieren, was sich möglicherweise aus der Tatsache erklärt, dass Mädchen von Jungen schikaniert werden oder nicht so leicht zugeben bzw. anerkennen, dass sie selbst schikanieren

Bullying nimmt im Allgemeinen mit dem Alter zu (Tabelle CO5.2). Wenn die Kinder älter werden und mehr Zeit in der Schule verbringen, verändern sich die BullyingVerhaltensmuster. Bei den Jungen nimmt das Bullying mit dem Alter deutlicher zu, vor allem in Griechenland, Luxemburg, Österreich und Deutschland. Nur die Türkei meldet sowohl für die Jungen als auch für die Mädchen einen Rückgang der Aggressivität mit dem Alter, obgleich die absoluten Niveaus in diesem Land vergleichsweise hoch bleiben.

In Ländern mit höherem Anteil 15-jähriger Migrantenkinder ist die Bullying-Häufigkeit im Alter von 15 Jahren größer (Abbildung CO5.3). Forschungsergebnisse deuten darauf hin, dass in Fällen, in denen Kinder in Gruppen schikanieren oder wo Bullying Bestandteil eines als normal geltenden Gruppenverhaltens ist, eine Funktion der Bildung von Freundschaften innerhalb der Gruppe und ihrer Aufrechterhaltung darin zu bestehen scheint, sich von anderen Gruppen oder Personen unterscheiden zu können (Duffy und Nesdale, 2008). Die Daten mehrerer Länder untermauern diese These.

\section{Weitere Informationen}

Atlas, R. et al. (1998), „Observations of Bullying in the Classroom“, Journal of Educational Research, Vol. 92, S. 8699.

Currie, C. et al. (2008), Inequalities in Young People's Health: HBSC International Report, WHO Regional Office for Europe, Kopenhagen, Dänemark.

Duffy, A. und D. Nesdale (2008), „Peer Groups, Social Identity and Children's Bullying Behaviour", Social Development, S. 1-19.

Hawkins, L. et al. (2001), „Naturalistic Observations of Peer Interventions in Bullying“, Social Development, Vol. 10, No. 4, S. 512-527.

OECD (2008), PISA 2006 Database, OECD, Paris.

\section{Anmerkungen zu den Abbildungen und Tabellen}

Tabelle CO5.2: Australien, Neuseeland, Japan, Korea, Norwegen, Irland, die Vereinigten Staaten, die Türkei und Mexiko fehlen in der Tabelle. Die Daten für Belgien beziehen sich nur auf den flämischen Teil. In den Daten für das Vereinigte Königreich ist Nordirland nicht enthalten. 


\section{INDIKATOREN FÜR DEN SOZIALEN ZUSAMMENHALT}

5. Bullying

C05.1. Jungen sind häufiger Bullying-Opfer und -Täter

Viktimisierung und Täterschaft nach Geschlecht, Länderranking nach gesamtdurchschnittlichem Verübungsgrad

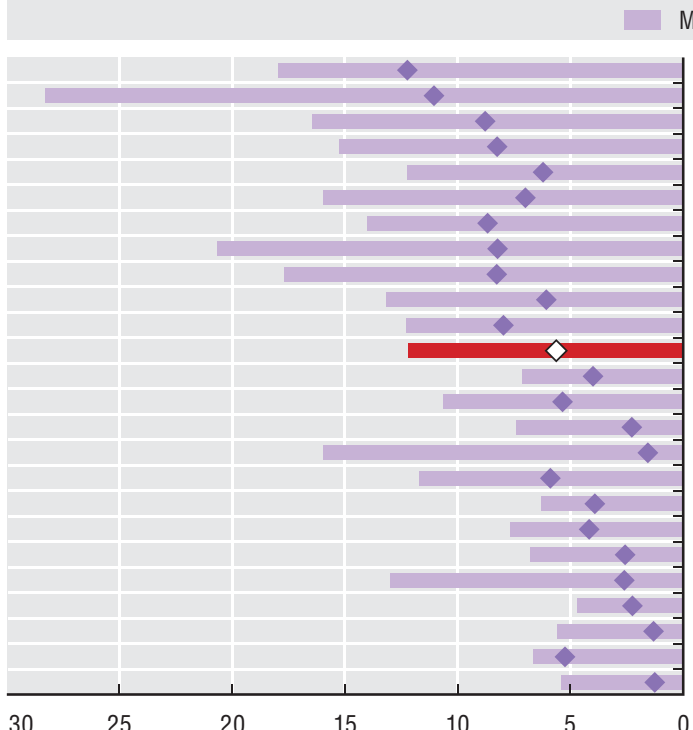

Kinder, die angeben, andere zu schikanieren (in \%)

C05.2. Bullying ist geläufiger unter älteren Kindern

\begin{tabular}{lrrr|rrr}
\hline & \multicolumn{3}{c|}{ Mädchen } & \multicolumn{3}{c}{ Jungen } \\
\cline { 2 - 7 } & 11 J. & $13 \mathrm{~J}$. & $15 \mathrm{~J}$. & $11 \mathrm{~J}$. & $13 \mathrm{~J}$. & $15 \mathrm{~J}$. \\
\hline Belgien & 6 & 6 & 7 & 12 & 11 & 14 \\
Dänemark & 2 & 6 & 5 & 7 & 11 & 15 \\
Deutschland & 5 & 7 & 9 & 9 & 17 & 21 \\
\hline Finnland & 2 & 4 & 2 & 5 & 6 & 8 \\
Frankreich & 6 & 11 & 8 & 13 & 15 & 18 \\
Griechenland & 8 & 13 & 12 & 16 & 28 & 38 \\
Großbritannien & 2 & 5 & 6 & 4 & 8 & 10 \\
Irland & 2 & 3 & 2 & 6 & 7 & 9 \\
Island & 2 & 1 & 1 & 5 & 6 & 6 \\
Italien & 7 & 6 & 5 & 13 & 12 & 14 \\
Kanada & 6 & 8 & 5 & 10 & 13 & 13 \\
Luxemburg & 7 & 9 & 10 & 8 & 16 & 24 \\
Niederlande & 3 & 4 & 5 & 11 & 12 & 12 \\
Norwegen & 1 & 1 & 3 & 8 & 5 & 10 \\
Österreich & 5 & 11 & 11 & 11 & 26 & 26 \\
\hline Polen & 4 & 6 & 6 & 15 & 14 & 18 \\
Portugal & 8 & 9 & 8 & 14 & 15 & 13 \\
\hline Schweden & 1 & 1 & 2 & 3 & 4 & 9 \\
Schweiz & 5 & 10 & 10 & 13 & 19 & 21 \\
\hline Spanien & 4 & 6 & 6 & 5 & 8 & 7 \\
Tschech. Rep. & 2 & 3 & 2 & 3 & 6 & 5 \\
Türkei & 16 & 13 & 7 & 21 & 19 & 13 \\
Ungarn & 2 & 4 & 2 & 3 & 7 & 10 \\
Ver. Staaten & 8 & 9 & 7 & 11 & 14 & 14 \\
0ECD24 & $\mathbf{5}$ & $\mathbf{6}$ & $\mathbf{6}$ & $\mathbf{9}$ & $\mathbf{1 2}$ & $\mathbf{1 4}$ \\
\hline & & & & & & \\
\hline
\end{tabular}

Source: Inequalities in Young People's Health: HBSC International Report (Currie et al., 2008). OECD PISA (2008).

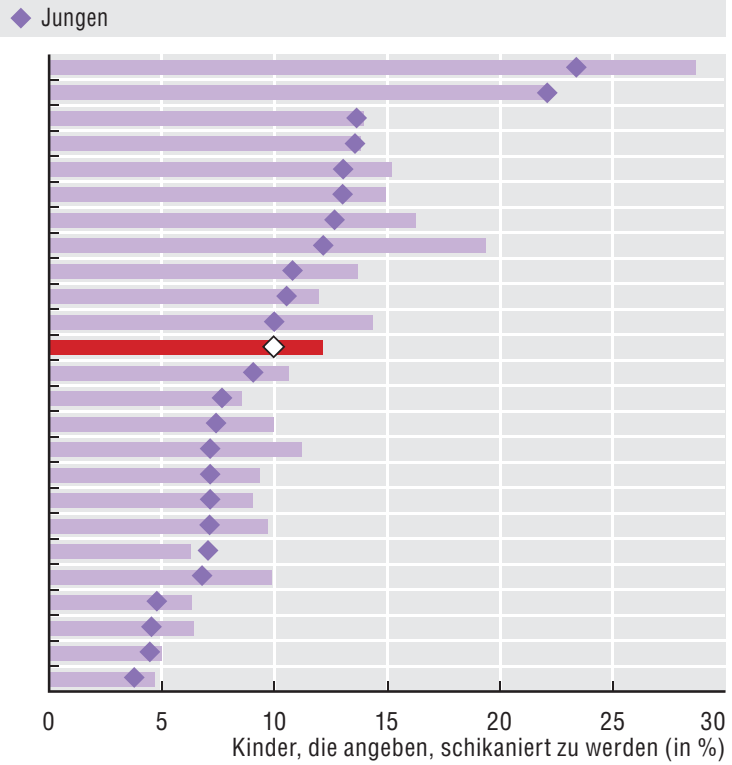

\section{CO5.3. Es werden mehr Kinder schikaniert, wenn der Anteil der Migrantenkinder der ersten Generation hoch ist}

15-jährige Schüler mit Migrationshintergrund (in \%)

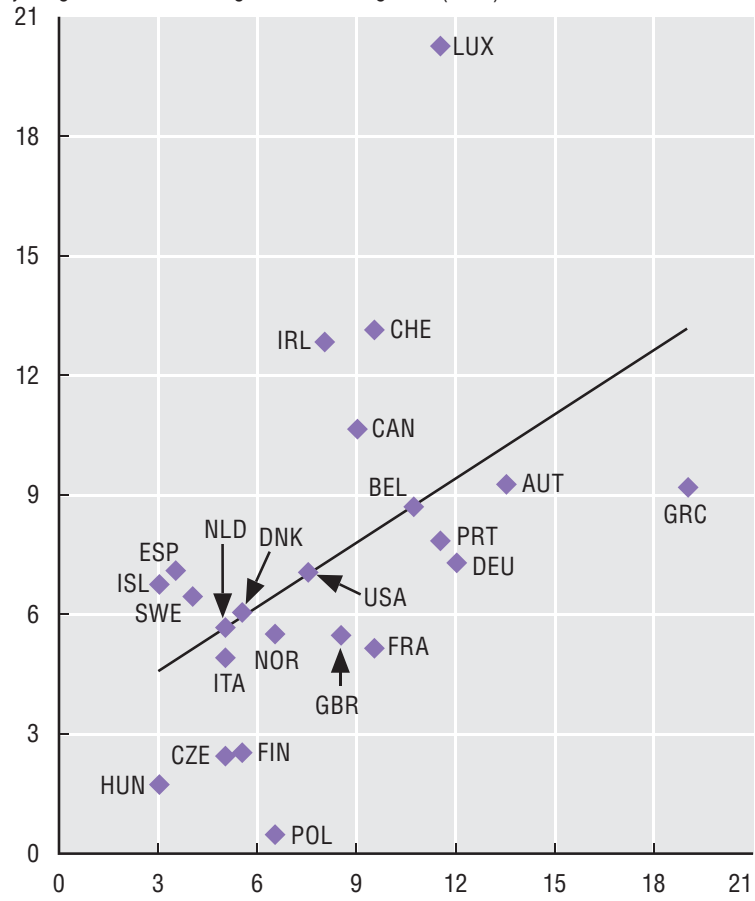

15-jährige Kinder, die angeben, wiederholt zu schikanieren (in \%) 


\section{Definition und Messung}

Unter Risikoverhalten von Kindern und Jugendlichen werden Verhaltensweisen verstanden, die eigentlich eher mit Erwachsenen assoziiert werden und die gefährliche Auswirkungen haben können. Das Ausmaß des Risikoverhaltens in den einzelnen Ländern zeigt, inwieweit Kinder angemessen betreut bzw. über altersgerechte Aktivitäten informiert werden. Zu den Indikatoren für Risikoverhalten zählt der Prozentsatz der Personen mit übermäßigem Alkohol- und regelmäßigem Tabakkonsum im frühen Jugendalter (Selbstangaben) und die Entwicklung dieser Prozentsätze im Zeitverlauf. Weitere Indikatoren für Risikoverhalten sind der Prozentsatz früher sexueller Erfahrungen sowie die Nichtbenutzung von Kondomen zum Schutz vor Schwangerschaft und sexuell übertragbaren Krankheiten (Selbstangaben).

Die der Berechnung der Indikatoren für Risikoverhalten zu Grunde liegenden Daten sind der Internationalen Studie zum Gesundheitsverhalten von Schülerinnen und Schülern (Health Behaviour in School-aged Children Survey 2005/06 - HBSC) entnommen, an der 25 OECD-Länder teilnehmen. Einige Länder haben es jedoch vorgezogen, Kindern keine Fragen zu Alkohol, Tabak und Sexualität zu stellen. Die länderspezifischen Schätzungen werden anhand der gemeldeten Prozentsätze und Stichprobenzahlen für 15-jährige Jungen und Mädchen berechnet. Bei den Trunkenheitsraten sind die Ergebnisse für die Alterskohorte der 13-Jährigen ebenfalls inbegriffen.

Eine beachtliche Zahl von Mädchen und Jungen raucht und betrinkt sich. Mädchen rauchen mehr als Jungen, doch betrinken sich Jungen häufiger. 2005/2006 war Rauchen unter den Mädchen in Österreich und unter den Jungen in Finnland besonders verbreitet, wobei Jungen in den Vereinigten Staaten und Kanada am wenigsten rauchten. Die Prozentsätze nach Geschlecht und Land reichten von 7-30\%. Bei der Trunkenheit war die Variationsbreite geringer. Am stärksten verbreitet war die wiederholte Trunkenheit unter Jungen im Vereinigten Königreich und in Dänemark; am geringsten unter Mädchen in Italien und in der Schweiz (Tabelle CO6.1).

Der Alkohol- und Zigarettenkonsum unter 15-Jährigen ist gegenüber dem Ende der 1990er Jahre verzeichneten Hoch rückläufig. Beim Risikoverhalten zeichnet sich eine Konvergenz zwischen den Ländern ab, da der Tabakkonsum unter den Mädchen und der Alkoholkonsum bei beiden Geschlechtern sinkt. Mit Ausnahme Griechenlands geben alle Länder einen rückläufigen Tabakkonsum für Jungen und Mädchen an. Der Anteil der regelmäßigen Raucher ist mit weniger als einem von fünf Kindern bei beiden Geschlechtern auf ein 10Jahres-Tief gesunken. Die Trunkenheit ist in Dänemark, Finnland und dem Vereinigten Königreich stark zurückgegangen, wo die Jugendlichen traditionell zu übermäßigem Alkoholkonsum neigen. Auf nationaler Ebene wurde nur in seltenen Fällen ein Anstieg der Trunkenheitsraten unter Jungen beobachtet. Fünf Länder - Frankreich, Italien, Österreich, Polen und Spanien - melden eine Erhöhung, die nur in Österreich und Polen gegenüber einem hohen Ausgangsniveau erfolgte. Auch unter den Mädchen geht die Trunkenheit zurück, was möglicherweise weniger erstaunen dürfte. In
Ungarn hat die Trunkenheit unter Mädchen allerdings zugenommen, ohne dass es zu einem entsprechenden Anstieg bei den Jungen gekommen wäre.

Ein Viertel der 15-jährigen Jungen und Mädchen gibt an, frühe sexuelle Erfahrungen gehabt zu haben (Abbildung C06.2). Die Schwankungen zwischen den Ländern halten sich in Grenzen, alle Länder bis auf zwei geben Quoten innerhalb einer Schwankungsbreite von $\pm 10 \%$ um den Durchschnitt an. Hingegen zeichnet sich bei den frühen sexuellen Erfahrungen eine recht deutliche geografische Spaltung zwischen den Geschlechtern ab. So ist der Anteil der Jungen mit frühen sexuellen Erfahrungen in den Mittelmeerländern am größten und der der Mädchen in den nordeuropäischen Ländern.

Ein Viertel der 15-jährigen Jungen und Mädchen mit einer frühen sexuellen Erfahrung verwendete beim letzten Geschlechtsverkehr kein Kondom. Die Daten zur Kondomnutzung sind begrenzt aussagekräftig, da nur 16 OECDLänder diesbezügliche Angaben zur Verfügung gestellt haben. Neun der an der HBSC-Erhebung teilnehmenden OECD-Länder haben es vorgezogen, 15-Jährigen diese Frage nicht zu stellen. Etwa drei von vier befragten Jugendlichen gaben an, bei ihrem letzten Geschlechtsverkehr angemessen geschützt gewesen zu sein, ihr Anteil reichte von $70 \%$ bis zu etwa $90 \%$. In nahezu allen Ländern verwenden Mädchen seltener Kondome als Jungen.

\section{Weitere Informationen}

Currie, C. et al. (2008), Inequalities in Young People's Health: HBSC International Report, WHO Regional Office for Europe, Kopenhagen, Dänemark.

\section{Anmerkungen zu den Abbildungen und Tabellen}

Tabelle C06.1: Die Daten für das Vereinigte Königreich beziehen sich ausschließlich auf England. Die belgischen Angaben sind ein einfacher Durchschnitt der Statistiken der flämischen und französischen Gemeinschaften für jede Erhebungswelle, außer die von 1997-1998, an der die französische Gemeinschaft Belgiens nicht teilgenommen hat. Der OECD-Durchschnitt wird unter Verwendung der für jede Welle angegebenen Daten berechnet. Der Zigarettenkonsum, der nur für 15-Jährige erhoben wird, betrifft Personen, die in der letzten Woche mindestens eine Zigarette geraucht haben. Die Trunkenheit erfasst den Anteil der Jugendlichen zwischen 13 und 15 Jahren, die angeben, in ihrem Leben bisher mindestens 2-3-mal betrunken gewesen zu sein (die Durchschnittswerte für alle Alterskohorten insgesamt werden anhand von Stichprobengewichtungen berechnet). Es wurde folgende Frage gestellt: „Haben Sie jemals so viel Alkohol getrunken, dass Sie richtig betrunken waren?" Australien, Neuseeland, Mexiko, Japan und Korea fehlen.

Abbildung CO6.2: Australien, Neuseeland, Japan, Korea, Norwegen, Irland, die Vereinigten Staaten, die Türkei und Mexiko fehlen. In Island, Luxemburg, Italien und der Tschechischen Republik wurden die Jugendlichen nicht nach der Kondomnutzung gefragt. Die Daten für Belgien beziehen sich ausschließlich auf die flämische Gemeinschaft. In den Daten für das Vereinigte Königreich ist Nordirland nicht enthalten. Zum Sexualverhalten wurden die 15-jährigen Jugendlichen gefragt, ob sie je Geschlechtsverkehr hatten. Die Frage nach der Kondomnutzung lautete wie folgt: „Haben Sie oder Ihr Partner beim letzten Geschlechtsverkehr ein Kondom benutzt?“ 


\section{INDIKATOREN FÜR DEN SOZIALEN ZUSAMMENHALT}

6. Risikoverhalten

\section{C06.1. Zigarettenkonsum und Trunkenheit unter Teenagern lässt gegenüber den Hochständen Ende der 1990er Jahre nach}

Raten des Zigarettenkonsums und der Trunkenheit von Jungen und Mädchen im Teenageralter, in Prozent, 1993-1994 bis 2005-2006

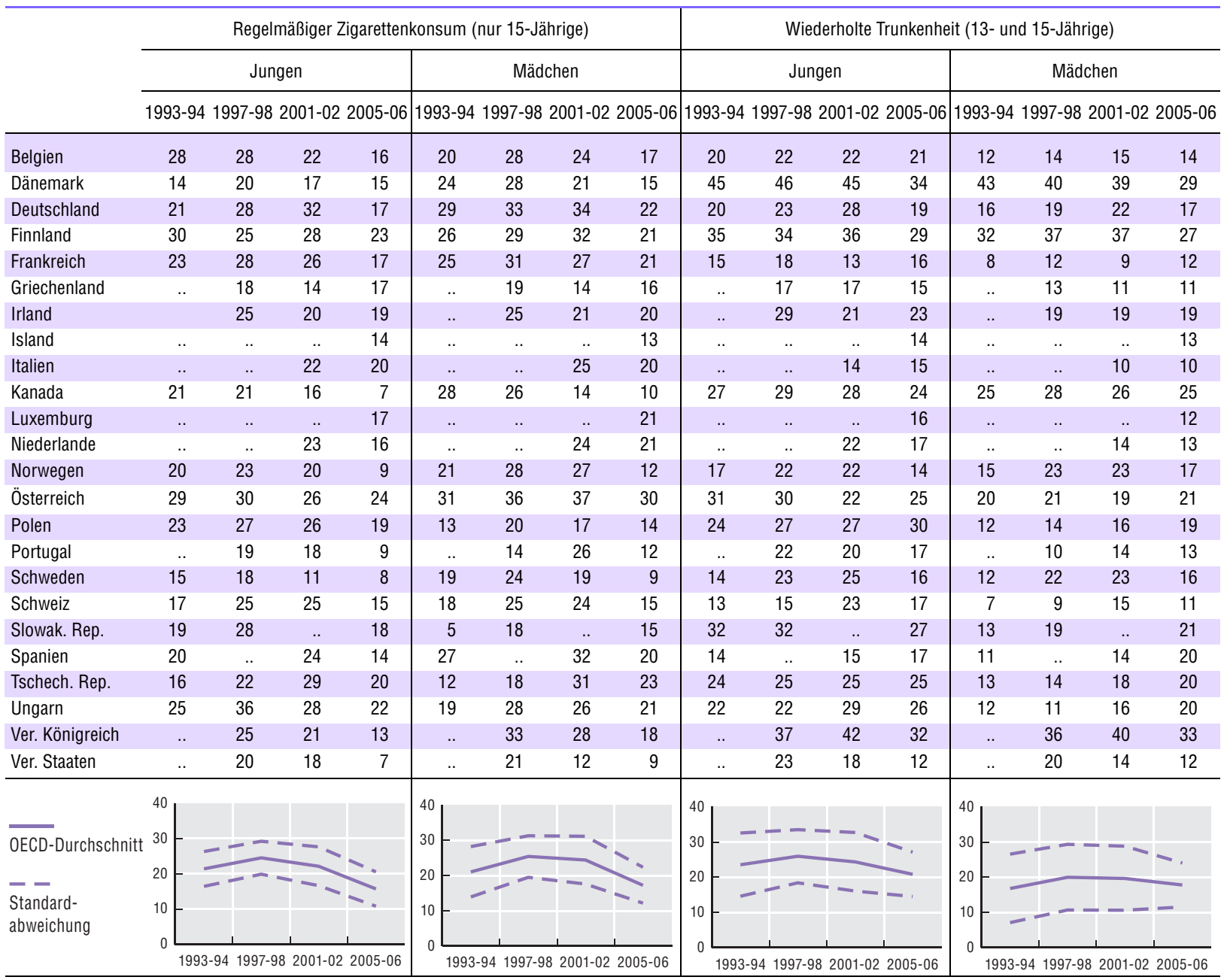

C06.2. Jeder vierte 15-Jährige hatte Sex, häufig ohne angemessenen Schutz

Sexuelle Aktivität und Kondomnutzung unter 15-Jährigen, in Prozent

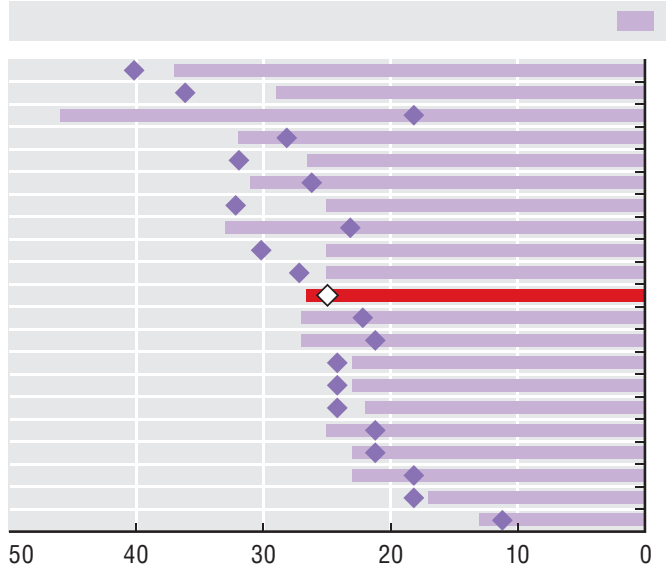

15-Jährige, die angeben, Sex gehabt zu haben
Jungen $\diamond$ Mädchen
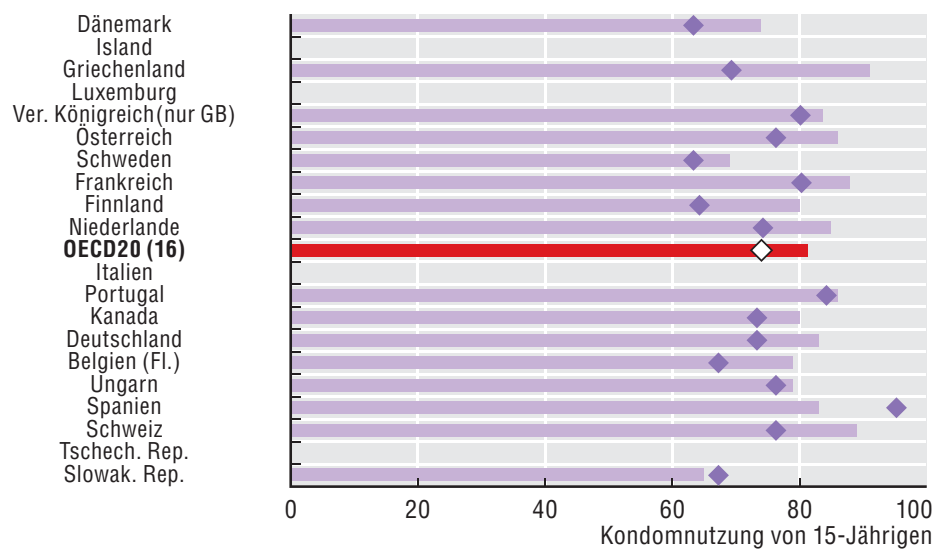

Source: Inequalities in Young People's Health: HBSC International Report (Currie et al., 2008). 
OECD PUBLISHING, 2, rue André-Pascal, 75775 PARIS CEDEX 16 PRINTED IN FRANCE

(81 200901 5P) ISBN 978-92-64-07570-2 - No. 569782009 


\section{Gesellschaft auf einen Blick 2009 \\ OECD-SOZIALINDIKATOREN}

Welche gesellschaftlichen Fortschritte haben die OECD-Länder zu verzeichnen? Wie wirksam sind ihre Maßnahmen im Hinblick auf die Förderung des sozialen Fortschritts? Gesellschaft auf einen Blick schafft eine Basis für die Beantwortung dieser beiden Fragen und bietet einen präzisen Überblick über quantitative soziale Trends und Maßnahmen im ganzen OECD-Raum. Die Ausgabe 2009 enthält ein breites Spektrum von Informationen über soziale Themen - z.B. Demografie und Familienmerkmale, Beschäftigung und Arbeitslosigkeit, Armut und Ungleichheit, Sozialausgaben und Gesundheitsausgaben oder Arbeits- und Lebenszufriedenheit - sowie einen Interpretationsleitfaden, der den Lesern helfen soll, den Aufbau der OECD-Sozialindikatoren zu verstehen.

Gesellschaft auf einen Blick 2009 aktualisiert einige in früheren Ausgaben enthaltenen Indikatoren und fügt zudem mehrere neue Sozialindikatoren hinzu, z.B. für die Körpergröße von Erwachsenen, die Selbsteinschätzung des Gesundheitszustands, Risikoverhalten und aggressives Verhalten von Jugendlichen (Bullying). Erstmals bietet der Bericht zudem in prägnanter Form eine Reihe sozialer Leitindikatoren, mit denen das soziale Wohlergehen in den OECD-Ländern erfasst wird. Ein Sonderkapitel untersucht überdies das Thema Freizeit in den OECD-Ländern.

www.oecd.org/els/social/indicators/SAG

Die Bücher, periodisch erscheinenden Publikationen und statistischen Daten der OECD sind in unserer Online-Bibliothek unter www.sourceoecd.org erhältlich.

Diese Veröffentlichung ist im Rahmen folgender thematischer Abonnements über SourceOECD verfügbar:

Social Issues, Migration, Health

Wegen näherer Einzelheiten bezüglich des Online-Zugangs zu OECD-Veröffentlichungen wenden Sie sich bitte an Ihre Informations- und Dokumentationsstelle oder schreiben Sie uns an SourceOECD@oecd.org. 